19. $)(x)(1$ mabeumd aftea ditin $x_{a}$ 





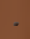




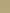

$$
\text { . }
$$




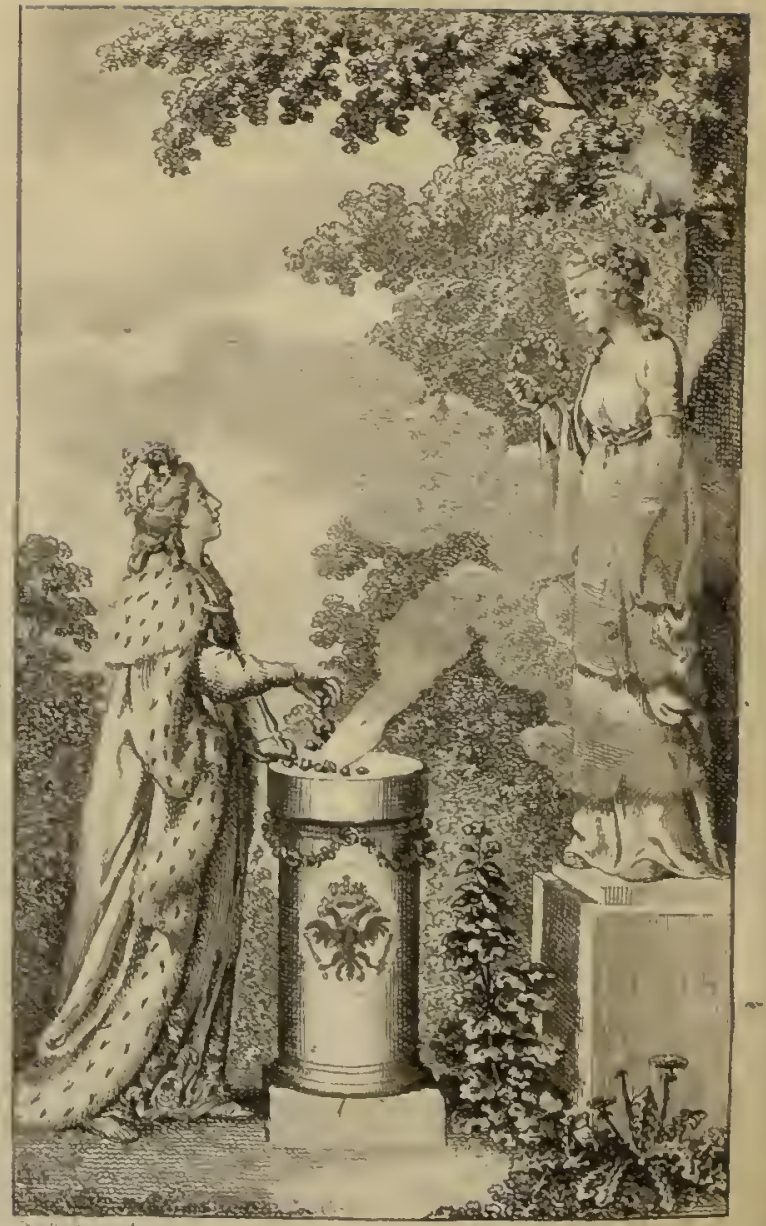




\title{
DEUTSCHLANDS FLORA
}

\author{
ODER \\ BOTANISCHES \\ TAS CHE N B U C H
}

ZWEYTER THEIL ,

IŨR DAS IA HR $279^{5}$

CRYPTOGAMIIE.

VON

GEORG FRANZ HOFEIIANN.

E R. I A N G I N

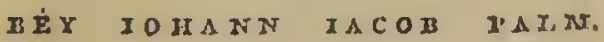





\section{Ex Libris \\ C. la CROLX. \\ ph.}

DIE ERKLAIRUNG DES TITELKUPEERS

findet man im ersten Theil des botanischen $\mathrm{T}$ a scheubuchs.

Einige dabei angebrachte Veränderungen beziehen sich anf die Iahrszeit selbst, in weicher Cryptogamen anfangen hervorzugehen und der Blumentragenden Flora zu folgen. 

A $\nabla$ CTORES BOTANICI

seculi XVIII.

VERZEICHNIS DEIR BOTANISCHET

S C H RIT T E N

des 18 Iahrhunderts.

Ahbildungen der Schwämme 3. I. 4. Berl. 1793. $\Lambda$ bercrombic's, I'ropagation and botanical arrangements of Plants and Trees. 2 vol. 3. Lond. 84 . Adanson, Histoire naturelle du Selsegal. 4. Par. 57. (Lcipz. 8. 73. Nachr. von scincr Pieise).

Afzelius, Ad. De vegetabilibus suecanis obserr. et experim. 4. Ups. 85.

Allioui, C. Auctarium id Flor. pedemont. 4. A. Taur. 59 .

Ialdinger, E. G. Ucber Litterar - Geschichte der theo ret. u. pract. Botanik. 8. IMarb. 94. Banks, I. Icon. select. pl. fol. Lond. 92. Jarbeu du Bourg, le Botaniste françois. 2. T. 8. Par. 67.

Barnades, M. Introductio in oryctogr. et zoo. log. Arrag. 8. Matr. 3!.

Bartram's, Will. Travels in the inter. parts of Nordamerica. 8. 92.

Batsch, A. I. G. C. Elenchus Fungorum, 4. Hal. Magd. 85. Cout. 1-2.89.

Banmgarteu, I. C. G. Scrtum lipsicum. 8. Eips. gn. Jeauvois, Pal. The first memoir of Olserv. on line plant denomin. Cryptoganick (Trans. of the $\Lambda$ meric. Soc. vol. $3.9^{3}$.)

Becker Thes. nuiv. r. herb. 8. Ang. Vind. 87. - - H. F. Beschrcib. der Bämme und Str. irn IMecklcub. 8. Rost. 9?.

Bellardi, L. Osserv. botaniche. 8. Torino, 88. Bellermanu, I. H. Abbild. zum Kalsinet d. vorzingl. Holzarten Fol. Erf. 98.

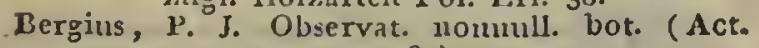
succ. 78.)

Berkculiout's Botauical lexicon. 3. Lond. 89. * 2 


\section{Classis et Ordo :}

\section{CRYTTOGAMIA MESCI (CALYPTIATA).}

Thadiculae numerosissimac, ex fibrillis patu. lis flexuosis, in tomeusum ab alibili tcrra vix separabile couflatae, fuscellac; aliae e surculi - partc supra terrac superficien erumpentes rariores, profundius coloratac.

Surculi erecti (a) simplicinsculi adscendentes, cespitosi, basi ctian ramosi, vel novas propa. gines, turionum al inirio speciem habcntes, agumt, quae debitam proceritatcu adeptac, de sua extremitate florcut, vel nucre feminei, yel mas. culi, distincei individui. Inferne surculi nul dinsculi ca qua spectant terram parte rutilant, sencim adsccudenuo fum dihntiores.

Folia inferiora breviora, rariora, adpressa, erecta, inde multo lougiora patula, recurva, lanceolata (b), serrata (c), carinata, transversim undata, nervo per uedium ducto notabili, pallidiore.

7\%los masculics in summitate crassioris surcul. Ji, rigidioris, disciformis; a foliis perigoniali. bus, extimis inaximis recurvis; inter luaec follimili spermatici (Stam. Antherae Hedv. cylindra. ceae uniluculares, apice dehiscentes, cjaculan. tes, pollen granulosum ; singulis filamentum bre. w, filiforme) perquam mimerosi $(\varepsilon)$, ante explosionem brevioribus, subin longioribus filis succulentis, (parap)/usibus) muniti. Absoluta -impraegnatione e centro surris imnovatio (more Polytrich.), sacpe in femine inm florem mutata.

Tlas'fernineus etiam in summitate surcuil sim. wlicis yel cjus divisionum intrit perigonium, c maximis folii, derum decrescentibus orimdus; 


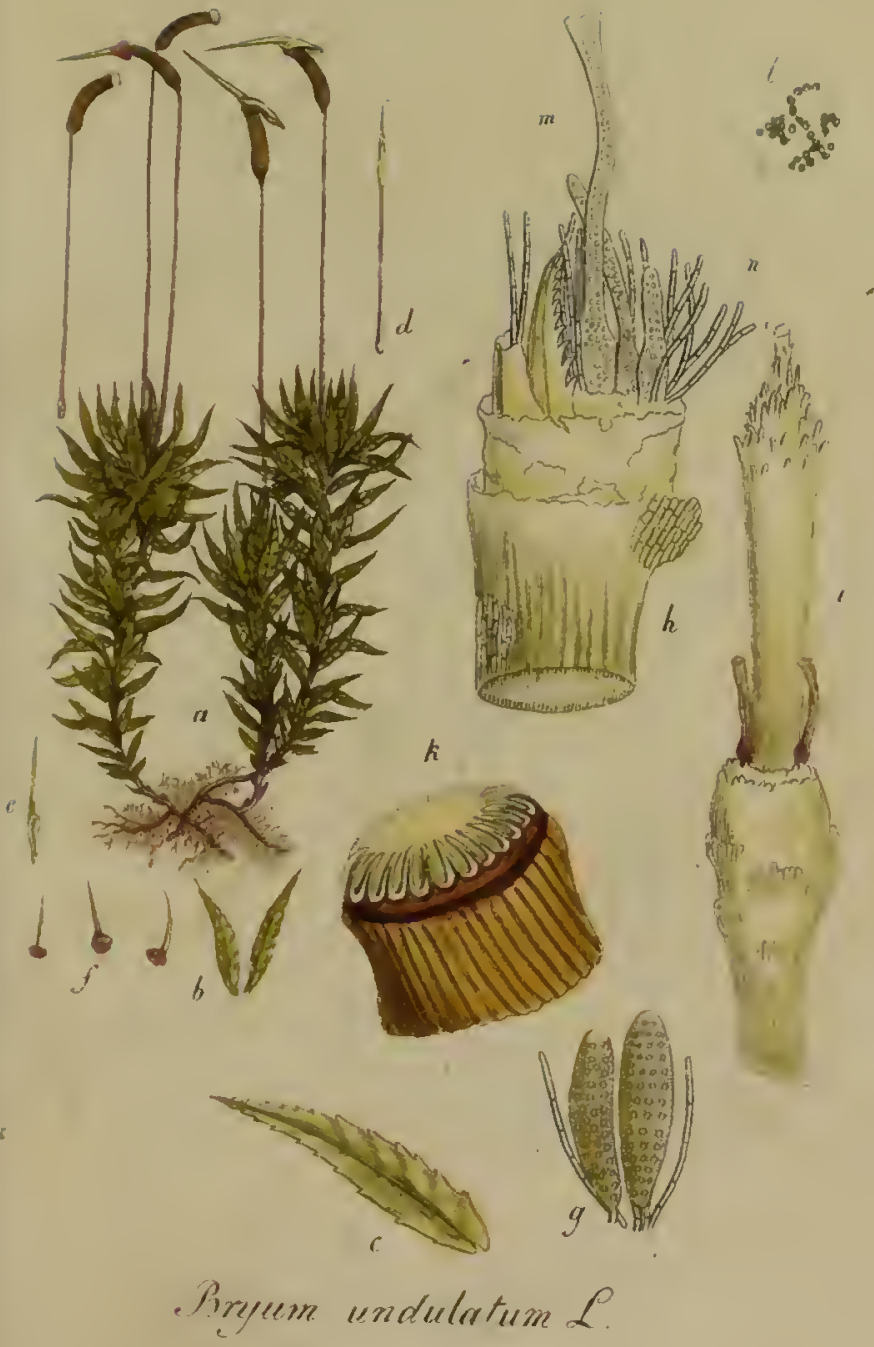





\section{A VCTORLS BOTA NICX}

\section{seculi XVIII.}

ENZEICIINIS DER BOTAINISCII E N S C H H IF T I N

des 18 Iahrhunderts.

Billardiere, la, I. I. Icones plantar. Syriac rar. descript. et observ. ill. 2. Dec. 4. Par. 92.

Blackstone Specimen botan. 12. Lond. 46.

Blond le, Catal. plant. e Cayen. niss. conscr. a I. Richard (Act. de la Soc. d? Hist. nat. de la Par. Par. fol. T. 1. 92.)

Böhmer, G. 11. Technisclie Geschichte der PH. 8. 2. Th. Leipz. 94.

Lolton, T. Filices britannicae. 4. Lond. 86.

Bondt, $N$. Verhand. over de overecuk. tusschen Dieren en Planten. 8. 92.

Bonato, G. A. Pisaura antomorplia. 4. Pad. 93. Morch, C. de Lettres sur les Trufies du Piemout. 3. Milan, 80.

Bordiga, B. Storia delle Piante forastieri - 4. Mil. 91.

Borkhausrn, NT. P.. Tentamen disposit. plant. Germ. seminifer. 8. Darmst. 92.

Bosc, L. I y y colp. axatuin Decum. sarment. (Act. de l.1 Soc. d' Hist. nat. T. 1.)

Bose, E. G. Pr. de nodis plant. Lips. $76-$

Bosseck, H. O. J) Antheris Hor. 4. Lips. 58.

Duchoz, R. I. Traite physique et occon. -

1Bradley, Rich. History of succulent Plants. 5. Dec. í Lond. 39.

Brez, I. La Flore des insectophiles. 8. Utrecht, gr.

Broussonet, I. I. V. Corona Aorac monspeliensis. 8. IMTonsp. gu.

Brusati, Catal. plant. hort. reg. bot. Ticiuensis, S. Pap. $9^{3}$. 
tribus, rarissime quatuor ganitalibus (m) filis. que succulcutis articulatis (ii) lorevibus ditatur, quorum 1, nounutuquam 2, rarins $3 \mathrm{vel} 4 \mathrm{im}$. praegnata repcriuntur; unde

Pedunculus (Scta) unins cjusdemquc perichactii, solitarius, getnclhts, tri-quadrimcllus. que crectus, ruljellus, rigidulus, siccitate con* tortus.

Vaginula (d) pyramidata, nuda, ore pe. dunculo ct

G)munocidio (tuberculo sctac) arctc adlacrens.

Cral.pptra mediac actatis (i) cylindrica, in summitate brevis:imis processibus muricata, (ge. silatia ad bitin sterilia: redrlectores Hedw.): znaturescentc capsula latcraliccer fissd, obliqua, glabra, acuminata (c) decidua.

Capisula (Theca Hedw, Pyxidium Elrh.) cylindrica, rircumsciss magis mimuse cerma, ce viridi demum fusca umilocularis; ad orifici apcrturam, vel porius

Operculi basin rleganter rubens; hoc tenuem subulan d corstum vel strsum directan emittic (f) dihutionis cotoris.

Amulus mullus.

Jeristoma (s. Peristominm) simplex (k), e denibus 02 angustis, dilute fuscis, linca alba parginatis, basi coalitis, apice

Fipipluramate, seu membramla tenui pli. catili imperforata connexis, exstructum est.

sporrangitimn (colmmella) longitudine fere capsulac, cylindricun.

Seminr. (1) Papaveris in modum per interstitia dentium sicca tempestate elabculia, intro sumt viridia, sghacrica, lacvia, demum fusca. 
A VCTORES TBOTA NIGT

seculi XVIII.

TERZEICHNIS DEIR BOTANISCIIEN S C II I I T E N

des 18 Iahrhunderts.

Bryant's historical acconnt of a Species of $\mathrm{L} Y$. coperdons. 8. Loud. 83.

Bulliard, Histoire des Champignons. Tom. I. fol. Par. 91.

Burmann, I. Indic. Flor. Jialab. et Herb. amb. fol. Amstel. 69.

- - N. L. De Geraniss. 4. L. B. úg.

Cavanilles, Ant. Ios. Icones et descript. plant. 2. vol. fol. Madrit. $91 \cdot 95$.

Canlinus; Ph. Zosterae occanicae anthesis. fol. Nap. 92.

Colbiürnsen, Progr. de sexu plant. fol. Hafn. 82. Compareti, Andr. Piscontri fisico - bot. ¿̈. Pad. 93.

Coulcii, A. Plantarum libri sex. 3. Bas. $9^{3}$.

Crantz, II. I. N. Institut. rei lierb. 2. vol. \&. Vien. 66. (Lips. 67.)

Croix, de 1a, Commbia florum. Ed. Rich. Clayton. 8. Lond. 92.

Culpepper's, Medical Herbal enlarged by Ha. milton. 3. vol. 8. Lond. 92 .

Curtis, W. Botanical Magazin. 88. Numb. \&. Lond. $87 \cdot 94$.

Cuttler, Man. An account of the veget. pro. duct in the Neighb. of Ipswich (IMem, of the Americ. acad. Vol. 2. 4. Hoston 85.)

Cyrillo, D. Furdamenta botanica. 2. vol. 8. 87. Darwin's Botanic Garten a Poem. 2. vol. 4. 9I.

Daucer, Th. Catalogue of plants in the botan. Gart. at lamaica. 4. 92.

Demidof, Proc. a, Enum. plant. hort. propr. rec. P. S. Pallas. 8. Petrop. 8 t. 


\section{Classis et Ordo:}

CRYTTOGAMIA HEPATICAE (MULTIBALVIA). sac.

Fradiculae vix ullae, brevissimae tomento.

Surculi inferne rudiusculi, cespites efformant densos pulvinatos, e vilidi zubiginosos, vel siccitate niavo - fuscos; adscendcutes, alterne ramosi: ramis vagis, anterins conlertis, incrassatis recurvis, subpiunatis; supra couvexi, sub. ms pliminsculi , e foliorum ciliis quasi villosi (a); feminci (b) breriores minus ramosi, semicall. sumpti, erectiusculi, apice unmerosis perim. thiis obsiti; alii steriles decumbentes longiores ramosioresque.

Foliola ordine duplici imbricata, superne couvexa (f) se invicem semilegchila recurva; superiora subquadrata, tri s. quadripartita (d c), inferiora Ianceolata (d), utraque inargine in mu. merosissimas lacinulas ciliares (quae per Ientem ex ovatis articulis compositac apparcut) divisa.

leriantia uumerosa terminalia, veutricosa lacvia, flabra, pallide subfisca, apice conniventia, quadridentata (ad lentem subciliata) Dasi foliis perichaetialibus cincta. gentei.

redunculi (Setac) tetragoni, pollicares, ar.

Cansula subglobosa, fusca, unilocularis, Iongitudiualiter dehiscens in valvulas quatnor aequales, patentes ollongas, obtusas persistenies. rentia.

Scnizza subfusca filis tortis clasticis adhae. 

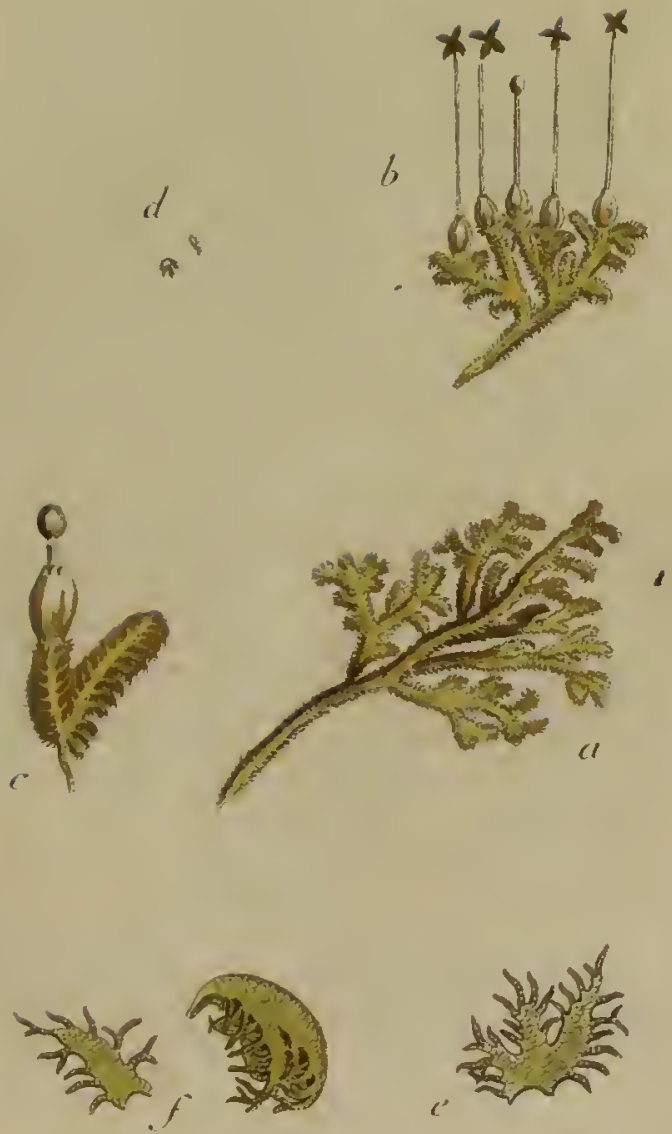

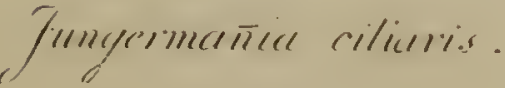





\section{AVCTOKES BOTANACX}

seculi XVIII.

TERZEICIINIS DER BOTANISCHEN S C Y RIITEN

des 18 Iihtrhanderts.

Pott, I. F. Harbkesche wilde Banniz. ed. 2. 8. g5: Ramatuclle, de, Descript. de la Chamómille d grandes fleurs (Jouru. d'hist. riautelle. ni. 18: 4. Par. 92.)

Reichel, G. C. De vasis plant. spiral. Lips. 58 : Reichard, I. I. Sylloge optisc. bot. 3. Fiancof. 82: Reiner, I. and Hohenvarth, S. Bot. Reiseri. 1. R. 8. Klagerti. 92.

Reitter, I. D. ind Abel, G. F. Abibildung lod deutsch. Holzarten - 4. Stuttg. 92 .

Relhaw, R. Flora Cantabrig. (c. trib. suppl.) 8. Cant. 85 - go.

Retzii, A. I. Florae scald. Prodr. 2. P. 8: Holm. 79 .

Reuls, C. F. Dictionarium hotau. - 8. Leipz. 81. Reynier, IMemoires pout scrvir a l' histoire nat. T. 1.8. Lausanue, 88.

Römer, I. I. Nenes ivtag. für die Botanik. x. B. 8. Zïrich, 9\%.

Hodschicd, E. C. Ver\%. merkwïrd. anf Guia. na wachs. Pf. (Bald. med. Iourn. 28. St:" N. Mag. 16. B. )

nooke's Descript. and sketches of remark. Oaks = 4. go.

Roth, A. TV. Botau. Abhandl. und B́cob. 4. Niirnb. 87.

Rotheram, I. the sexes of plants vindicated. 8 .

Rotrï̈ll, C. F. Descript. rar. plant. 4. Hafn. 78 . Roucel, Traite des plantes - qui croissent na. tur. dans les env. des villes de Gaud d'Alost etc. \&. Liegc', 92. 


\section{Classis et Ordo:}

\section{ERYPTOGAMTA FUNGI (HYMENOCANTA)}

Expansio mollis fibroso - lannginosa in cor. tice vel ligno, sine ordine, ad marginem de. pressior araneosa, medio pulvinata, amoene car. nea vel pallide rossa, sub qua latitat farina concolor, quae lente snbjecta ex acervulis conglo. meratis interspersis filis constare videtur. Haec intra aquam in minuta grannla pelhucida dissol. vintur. Aliam observo plantam Stilhosporae si. milem in cortice Fagi, compactiorem, sparsain. vel conglomeratam, ejus dem penitus coloris. cujus seminiformi: conpusula so Besimiura, ad lentem majora oblonga et quasi lomentacea, seu septilo in duas partes divisa; fila ex contorsio. ne articulata.

STIIBUM HIRSUTUMV

\section{Classis et Ordo:}

\section{CRYPTOGaMta FUNGI (MUCORACEA).}

Intra rinosum corticem Mucores persisten. ses sine ordine sparsi.

Stipici ochraceo capillari, setis erectis deci. duis hirsuto, selusim atteunato, insidet

Capitulum globosum album golidum diapha. num. Substantia sicca fragilis. Farina fibrosa hinc inde substrata. 

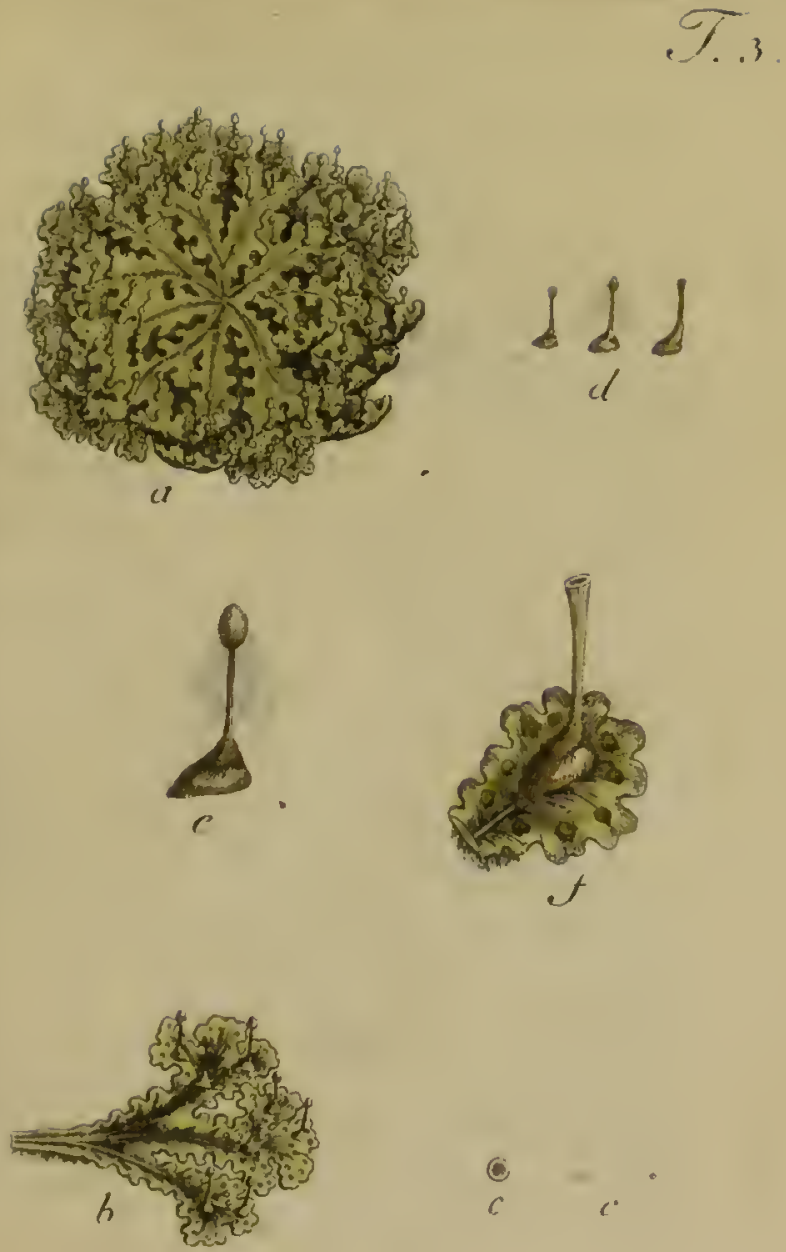

- Thlafice piufiller. 



\section{A V CTORES BOTANICI}

\section{seculi XVIII.}

VERZEICIINIS DER BOTAIISCHEN S C H I I F T E N

des 18 Ialirhunderts.

Fionssean and Martyn's Letters on the Elements of Bot. Ld. 3. S. Lond. 91.

Roxburgh, Nerimu tinctor. (Dalrymple orient.

Repert. 4.Lond.g1. Asiatick liescarches 4. Calcutta, 92.)

Rozier, Deinonstrat. clement. de Bot. 3.t. g. Lyon, 87 .

Ruiz, Hipol. Quinologia. MTarr. 8. ge. (Della Chind. 8. Roma, 92. Von dem offic. Fic. berrindenbaum. 8. Gott. 94.)

Russel, Alex. the Nat. Hist. of Aleppo. 4. Lond. 56.

- Parr. An acc. of the Tabasheer (Phil. Trans. v. 80.4 . 8n.)

Putty's Essay towards a mat. hist. of the county of Dubliu. 8. 2. vol. 72 .

Sabbati, L. Synops. plant. in solo romano -

4. Ferr. 45.

Salisbury, Icones stirp. rar. fol. Lond. 9ı.

Saunders's, short and easy Introduct. to scientif. and philnsophic Bot. 8. Lond. 92 .

Scanagata, I. Syst. veget. Ed. 15. 8. Pdv. Schaeffer, I. C. Botanica expeditior - 4. Ra. tisb. 60.

Scherer, I. A. Beob. übcr das phanzenähnliche

Wesen in deu warm. Karlsb. Wässern. 40 Dresd. 83 .

Schinz, C. S. Ioh. Gefsners phytograph. Tafeln. fol. Zirrich, 9i.

Schkuhr, C. Botan. Hand b. 14. II. 8. (1. 209.)

Witten b. $90 \cdot 9$ \%

Schleicher, I. C. Cent. pl. exice. C. 3. fol.g' 


\section{Classis et Ordo:}

\section{r.RYPTOGAMIA FETGT (UTHICULOSA).}

III aversa pagina foliorum Ancmoucs pumcta lutesccutia distucta solitaria, adspersa, quae accuratius inspecta

Aggedulae sunt ab initio gramulis mim. tis gloljosis, semiglobosis similcs, membraumla coctis, demum iu scuncupulam apertis intusque Utriculis mudis concoloribus, vel rufescentibus, ad lentem subrotundis pellucidis, massa granuIosa refertis.

\section{A ECIDIUM RUMICIs.}

\section{Classis et Ordo:}

CATPTOGaita FUNGI (UTRICULOSA).

Tolia Rumicis aquatici, maculis in aversa pagina notantur rotundis variis, variacque mag. nitudinis, planinsculis, per ambitum rotundis et magis depressis vel sine ordinc confluentibus, amocue saupruncis, ctiam pallidiorc colore va. ricgatis. Hae ex

degedulis dense et orbiculatim confertis, contrifugis componmunr, per ambitump in pustulas elcvatis. Cuticula di:parere iscipit, aperimutur Aggednlac, ore retracto denticulato pallesccute.

Besimina (sen Utricnli seminales in sp)stiis disci contenti) unda concoloria, neque fabrica a priori diversa, in granulosam massam demum dissoluta. 
A TCTORES, BOTANIEL seculi XVIII.

TERZEICIINIS DET BOTANISCHEIV S C H R I T TE N des 18 Iahrlunderts.

Schmidel, C. C. Ic. plant. ct anal. part. 3. m. (t. 75.$)$ fol. Erl. 95 .

Sclumidt, F. W. Flora bocmica. 4. C. fol. Prag. - - Fr. Oestreichs allg. Baumz. \&. B. fol. rien, 9\%.

Scholler, F. A. Suppl. Flor. Garb. 8. Barb. 95: Schrader, H. A. Spicil. Fl. germ. 8. Hamov. 94: Schrank, Fr. d. P. Primit. flor. salisb. 8. Frat. Schreber, I. C. I. Spicileg. Florac lips. 8. Lips. 71 .

Sclnwegman, F. et G. V. Schneevogt Ic. plant: rar. fol. Haarl. 92-93.

Scopoli, I. A. Dissert. ad scient. natur. part. P. 1. (plant. subterran. descript. et delin.) S. Prag. 72 .

Scnnelier, I. Encyclop. meth. physiologie ve. getale - 4. Par. 91 .

Segnier, I. F. Bibl. lor. 4. I. B. 60.

Smith, B. Barth. A botan. descript. of the Po. doph. dinlivll. ('Trans. of the amer. Soc. V.. III. $4 \cdot 930)$

- I. E. Car. Limnaei Flora Lappon. Ed.ء. 8. I, กnd. 92.

Sonnerat, P. Yoyages aux Ind. orient. et a la. Chine. 2. T. 4. Par. 82.

Sorverby's, I. Euglisl Botany. \&. vol. 8. Lond. Spielmann, I. R. Olera argent. 2. D. 4. Argentor. 76 . 


\section{Classis ot Ordo:}

\section{CEYHTOGAMIA FUAGI (LOMENTACEA).}

Sphaerulae gregatim nasci solent ligummque Salicun caveruosum incolumt globosae, ninia. tae, actate cocciueae, maguitudiue seminis Mi. lii; pellucentis et carnosac mbstantiae. Ad ba. sin fibrosim breve tonenum non raro per lentem observatur. Ex orificio tenui farinosam massam in fila contortam rmittumt; exsiccatione subsidente ac corngato ore, inde sacpe conca. vae, et ab ejecta farmosa materia glusi comsjersae. Halec ad augnecuta lentis considerata et aqua diluta, ex innumeris

Lomenlis sen articulatis leguminibus compouitur, radiatim intra fibrosan gelatinam divergeutibus filiformibus, articulis ipsis oblougis pellucidis subiubricatis, dispermis, sponte secedentibus.

\section{MUCIIAGA RETICUIATA,}

\section{Classis et Ordo :}

\section{CRYPTOGAJIA FUNGI (BYSSACEA).}

Ambigit inter Fungos et Byssos, arancosa cxpansio in disco reticulatim fibrosa, candidissina. Spatiola intra reticulum depressa, angue losa variac magnitudinis ac figurae, pulvere te. nni respersa concolore, ad lentem pellucido granuloso. Auctumuo post pluvias ad truucos putrescentes, Exsiccatione nou mutatur. 


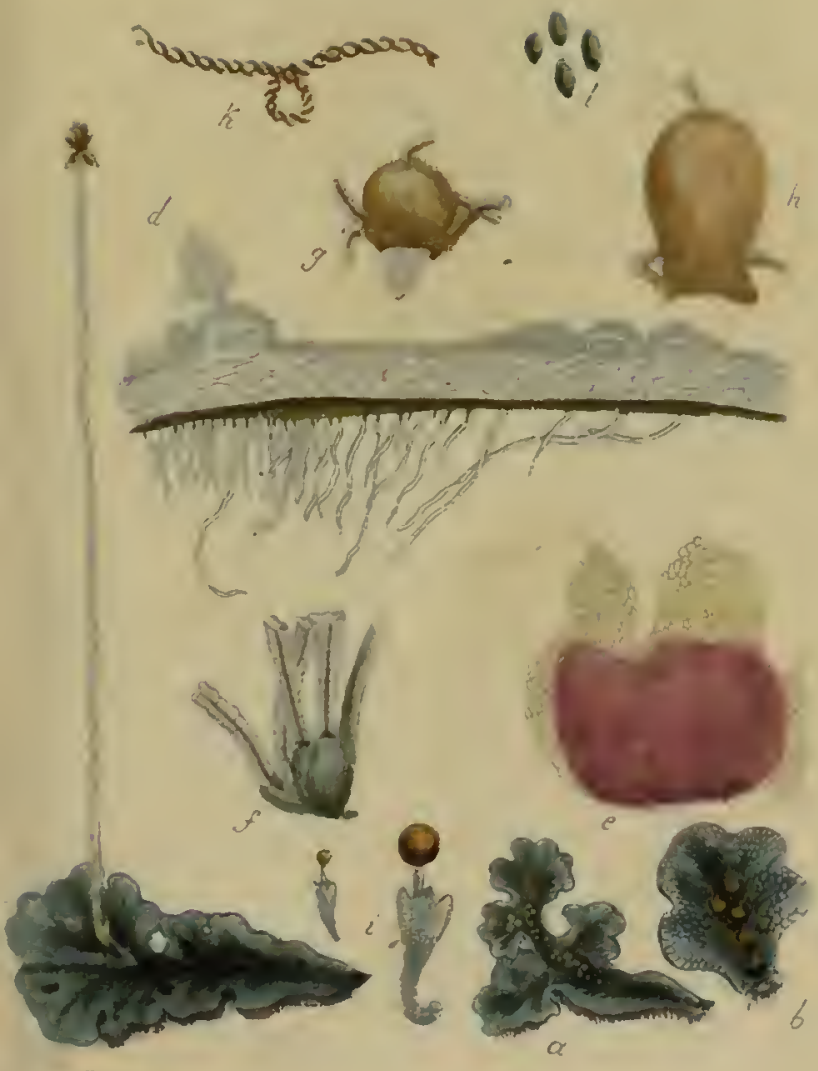

$r$

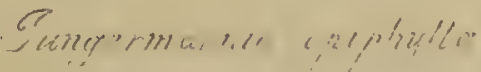





\section{seculi $X V I I I$.}

ERZEICHNIS DER IOOTANISCIIEN SCH H IF TEN des 18 Ialwhunderts:

sprengel, C. K. das entdeckie Geheimnifs der Nat. in der Befruchtung der Blumen. 4 . Eerl. 93.

Stecles, Essay on Gardening. 8. Lond. 95. Stcphani, F. Enumer. stirp. agri IMoscov. 8. IMosc. 92 .

Stillinglieet's, B. Tracts ou Natural History. $8 x$ Lond. 91 .

Stöver, D. II. I.eben des Rilters C. V.-Linué. I 2. Th. 8. Hamb. 92.

Ström, H. Ueber cinige scltcne Moosarten'in: Norvegen (Schrift. der Naturforsch. Ge.. sellsch. zu Kopenhag. aus dem dän. 1. $13 \%$. Kopenh. 93.) Suckow, G. A. Diagnose der Pllanzengatt. E. Lcipz. 92 .

Swartz, O. Icon. plant. incogn. Ind. occident. fasc. 1. fol. Erl. 94.

Thuilier, Flore des environs de Paris.8. Par. go. Thumberg, C. P. Resa uti Eur. Africa, Asia Ђ. T. 8. Ups. 89. (Deutsch. Ucbers. Berl. 92. 8.)

Tillesius, G. G. Musae parad. icon. 8. Lips. 92. Timm, I. C. Vorläutige Nachlese zur Flora me. gapolit. (Mag. fiir dic Naturk. u. Ockon." Meklenb. heran:geg. von A. C. Siemfsen. x. B. 8. Leipz. 92.)

Tode, H. I. Fungi mecklenburg. select. 2. F.: 4: Lu11. $9^{2}$.

Tozzetti, G. Viaggi - 12. T. 3. Firenze 79. Trattimick, L. Flora anstriaca sicca. C. b. fol. vien. $9^{6}$. 
DEMATIUM VENIICIIIATUM.

\section{Classis et Ordo:}

\section{CHYPTOGAMIL ALGAE ( BY:SACEA).}

In puerescentibus stipitibus herbarum tenuissima ac subcinerca, quasi prouis obductis, Byisus per leutem ostendit fila (sen stipites) iuferius nuda erecta, simplicia, superius verucillatis et articulatis ramulis in verticillo fere $3-4$, cince, versus apicem approximatis, breviori. bus, pellucidis, persistentibus.

\section{IEMATIUMT ANTENNAEFORME.}

In arborum laesis trumcis nigerrimac ìnaculae quasi ex pamto sericeo, louge liteque extellsac, quac ad lentem vitream examinatac, fiblis componuntur sinyplicibus articulatis fusco nigris : articulis oblong s, aqua pelluccutibus, in nicdio opacioribus, facile discedentibns. Vel confervas articulatas cum Byssis conjumit, vel ad lomeu. tacea praesertim. Stilbospora pertinet.

6IIBOSPORA ASTEROSTORA.

\section{Classis ei Ordo:}

CRTPTOGAMIA FUNGI (LOMENTACEA).

Macuiá nigrae variae, liguratae, radiàtae serpentinae, conlluentes, ex chitsis sub cpidermide corticis

Lomenlis compactis undis comlatac. Haec ad vitra aurentiora apparent triangulara stella. rum in modum radiata: 2adiis 3.4 ; ex lomentis varue connatis sibique impositis, oblongis, yer transversa septụla $(5-4)$ in loculamenta fivisis, demum intra aquan turbinatis inque gramulo. sam massam dissolutis. 


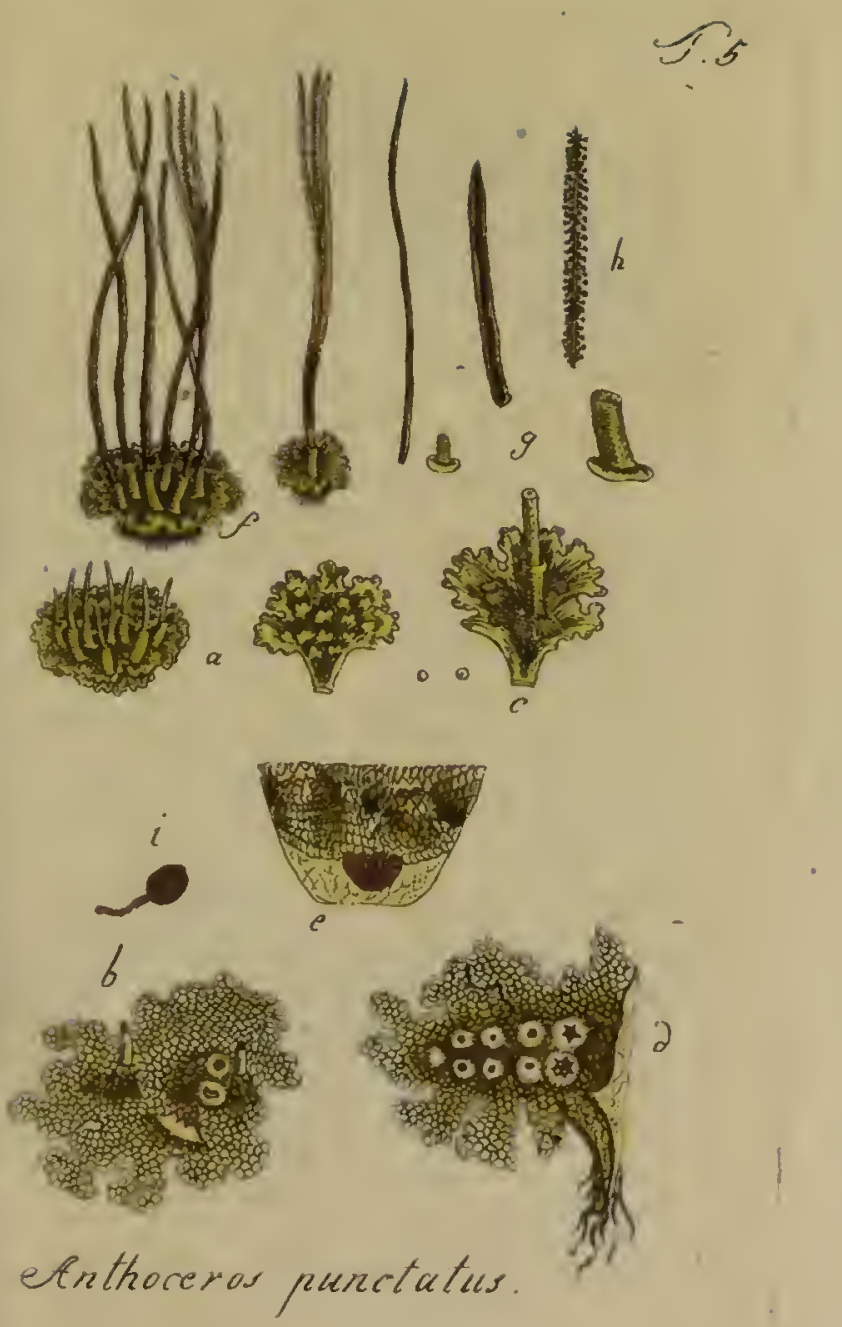





\section{A V C TORES BOTANICI}

\section{seculi XVIII.}

VERZEICIINIS DER BOTANISCIIEIN

SCII RIFTEN

des 18 Iuhrhunderts.

König, I. G. Descript. Epidendror. (Rerz. Fasc. Krapf, observ. bot. V1. Fol. Lips. 91.)

Lachenal, W. de, Emend. et Auctar. ad Hal. leri hist. stirp. helv. spec. 1. (nov. act. helv. v. 1. 4. Basil. 87.)

Laicharding, I. N. Mannale botanicum. 8. Lips. Latourrette, A. L. 94.

Tecrs Avign. 70.

Avign. 70. at mont. Pilat. 8.

Leers, I. D. Flora herbornens. 8. Berol. 89.

Lconhardi, G. F. Naturgesch. firr alle Stände:

Linné, Car. a, Genera plantarum. Ed. octava

(c. I. C. D. Schreber) 2: vol. 8. Franc. ad IVIocn. 91.

- - - F. Dissert. botanicae (amoexit. ilieblacad. vol. 10. 8. Erlang. 90.)

Eilieblad, S. Swenska ört - slagen - 8. Ups. 91. tinck, H. F. Annalen der Naturgesch. 2. St. Gott. 94 .

Löwe, I. C. C. Physik - ökon. Bem. über das Riesengeb. (Oekon. cam. Schr. 2 T. 8. Bresl. 89.)

Loureiro, I. d. Flora cochinchinensis. 2. T. 4.

Ulyssip. 90. (Ed. Wild. Berol. 9. 93.)

Iudvig, C.F. Ep. de pulvere Anther. Lips. 78.

C. G. Ectypa vegetab. 8 fasc. fol. Hal. Sil. 64 .

zMastcus, I. F. Ver\%. der österreich. Bäume,

Staud - and Buschgew. 8. Wien, 81. 
A RCIRIA IEUCOCEYHALA.

Classis at Ordo:

CRYPTOGAMIA FUV̈GI (HYMENOCARPA).

Folionum loagi superficici exsiccatorum in.. mascumtur capitula scu

Aggedulue pyriformes e rufescenti spadi.. cea testa conflatac, rigida; a medio superjora versuls attcnnatur trista in cuticulam fragiicm । obsolcte albam, aliquantum farmosam, quae va.. rie dehiscit et commimi incipit.

Stipiti insident rigido lineari subfusco de.. mum nigricante, ejusdem fere cum aggedulis: longitudinis, cleyanter jnter se coordinatis. Dirs: satis integrae iu foliis remavere solent, successut vero temporis, vol acu privata particulatin ab-. oliur a medio ad rerticem tencrrima cuticula, atclue interiora pateficri incipinnt, quat primo" intuitu ex mera farina componi videntur. Ocus: lo vero armato duplex adparet substantia, testia nempe et solutum ab ista ovuli oblongi snb specic

Cixpillitim s. reticulum candidum, rigin. diusculis et brevissimis turbinatis filis compa-. ctum, cum interioti ct inferiori paricte testac: coharens. Nec excutitur reticulum ex ipso pa.. ricte inferiori, sed impalpabilis pulvișculıs, nu.. do oculo ex meris globosis solidisque uigris gra.. nulis compositus, seu

Besimina in spatiis reticuli vacuis conten." ta et sensim excussa, circa testae inferiorem solidiorem et durabiliorem partem quasi intera ca. lycem potissimum discumbunt.

THXLEPHORA COLLICVIOSA. Classis et Orda:

CHYPTOGANLA FUNGI (HYMENOCARPA).

Ad ramos emortuos crusta varie ct sine ordine in maculas minores scu majores, conflu. cutes, undatim expansa. Superficies inonticuldsa, vernicosa, polygonia rimosa, ex carneo pallida, pruinosa. Colliculorum color fit ru. fesccus, croceus cinuabariuls, humectatr vel detritionc. Snbstatia temax coriacea, madefa. cta mollescit fungosa. 
T.
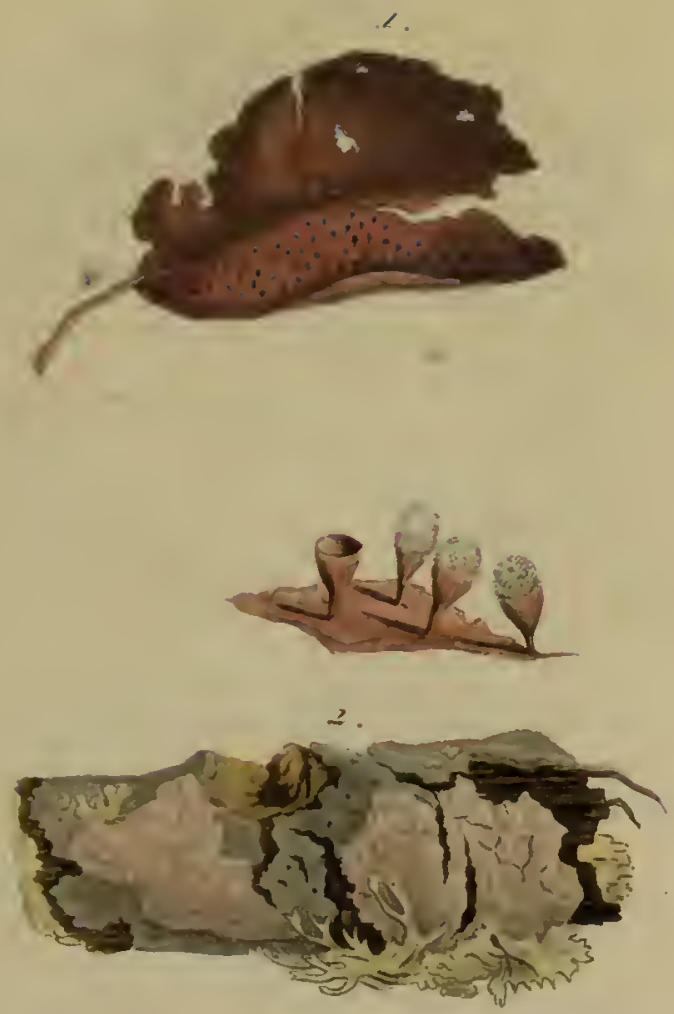

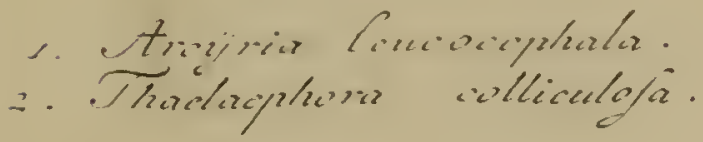



A V C TORES BO TA I I C I

seculi $X V^{\prime} I I I$.

ERZEICIINIS DEIR ISOTANISCHEI SC II HI I T E N

des 18 Iahrluunderts.

Magazin, botanisches, heransgeg. von 1. I. Räner nnd $\Gamma$. Usteri. 4. R. 8. 9 t.

allaratti, I. F. de vera for. exist. in plant. dor.sif. cd.2. (Nuova 1haccolta d'Opuscoli filo. lof. T. 20. ) 8. Rom. 6 s.

Narck, de la Encyclop, meth. Botan. 3.T. 4. Planch. ( 200$)$ de la Lotall. prem. Livr. par. 91.

MIartcli, N. Braschiae plantac nov. gen. descr. 4. Pom. 91.

Marterstock, I. C. Womiscine Flora. 8. Bonn. 92. Martius, VV. E. Gesammlete Nachr. über deı macassar. Gif̂tbaum. 8. Erl. 92.

Martyn`s, I. Hist. plant. rar. fol. Ed. germ. Norimb. 94.

Martyı, Th. The Lallgnage of Botany. S. Lond. 93 ."

Mattuschka, H. G. v. Emunerat. stirp. in Siles. sponte nascent. 3. Vratisl. 79 .

Mayer, I. Samml. plyys. Aufs. die lömische Na. turgesch. betreffend. 4. Th. 8. 9t - 94.

- - P. 1. Pomona Francon. 3. T. 4. Nïrnb.

- I. C. A. De varis plant. (IVcm. de I'A. cad. des Sc. et 13.1. de Perlin, 4. 93.)

ATedicns, J. C. Critisclie Bemerk. über Gegenst.

ats dem Planzenrciche. 2. St. 8. MLamuh. 93. ancen's, Mifs. Exnlic plants of the Royal Gar. dent at Kew. 2. N. fol. Lomd. go.

IiTcese, D. Flora Frisica. 3. Franeck. 60. GMeyer, B. Scmina (propr. Hort.) 8. Hanov. 9\% Theyrick, IV. The nesv Tamily-Herbal. 8. Lond. 91 .

*** 2 


\section{CIATARIATRIAIA}

\section{Clessis et Ordo:}

CETTOGavis FẼG (UTRICtzosa).

E putresceute liguo Clavulae prorumpunt simplices.

Lapioulo instnctae oblongo in obmsum apicem altenuato. erecto vel obliquto simplici ut plurimuas, rarius duplican vel diviso, sub. inde de compressusculo aut piicato - stucato.

sipes brevis teres. Substmua mitorrais carnoso - gelatinosa. Color obsolete lutesceus, exsicraticue resinosus. Prunordia hujus plantae clavam simplicem tererem, apice plus mimuse obmane rel acuminatan longiori insidentem itipite, imitari soleut.

\section{CIATARIA ACROSPERMUX.}

\section{Classis et Ordo:}

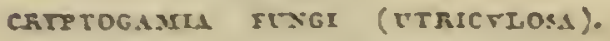

Ad stipites herbanta exsiccatos clarulas simplices sparsae, pistilli iuversi forma, cra. sนim minutisimae. subobliquae, surstur mo. diсиm quanum amplizutu in

Capirulum obusum, elegauter rosci colo. ri: vesirulis per leutem fariuosis quasi sacclaro obducturu. nosa.

scipes brevis pellucidus. Substautia car. 


\section{T. 7.}
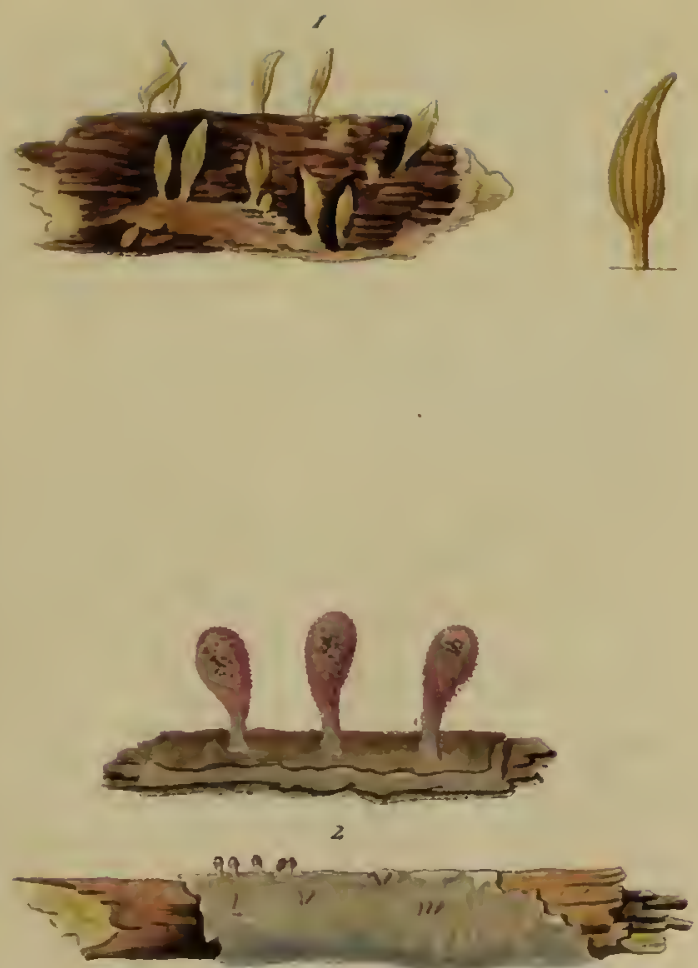

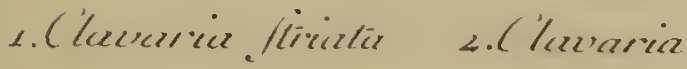
- Acrelpermeem. 

A VCIORES BOTANICE

seculi XVIIL.

VERZEICHNIS DER BOTANISCHEN SC H R I F T EN des 18 Iahrhunderts.

Jlichant, M. A. Notice de quelques plantes observées dans l'Amérique Septentr. (Journ. d' hist. Mat. T. 1. 4. Par. 1792.)

Micheli, P. A. Catal. plant. hort. Florent. fol. Flor. 48.

Milne, C. and Al. Gordon's Indigenous Bota. ny - 8. Lond. 93.

iVillers, 1. Illustr. of the sex. Syst. of Liu. 2. vol. Lond. 79. (Edit. Germ. c. Weis Frankf. 8g.)

- - I. F. Fignres of non descript. Quadr. Plants. 10. N. fol. Lond. 93 .

Miller, Ph. Figntes of pl. descr. in the Gardn. Dict. 2. vol. fol. Lond. 60. (Ablild. der sel. tensten Pfl. seines Gartu. Lex. 2. Th, fol. Nimrub. 78.)

Moench, C. Meth. Plant. - 8. Marb. 94. inühlenberk, H. Index forae lancastr. (Trans. of the Auner. Soc. Y.III. $4 \cdot 9^{3}$.) Miller, O. F. Eftcrrättning om Svampe. 4. Co. penh. 63.)

Murray, I. A. Gar. a Linne Syst. Veget. Ed. 14 Mutis, I. C. Batschia nov. Gen. (nov. Act. R. S. Ups. vol. V. Ups. 4. 92.)

Nanmburg Ehscheid. der Orch. (Au. d. Bot. 9. St. 8. 9\%.)

Necker, N. 1. d. Trajié sur la Mycitologie. 8.

Nenenhalu der Iiungere, bot. Bcolacht. (Ehrhart's Bcitr. 6-7. B. 8. Hanov. 92.) Nocca, D. Observ. bor. (11. An. d Bot. S. St.) Nomenclator Fungor. P. 1. Agnrici. 8. Berl. 39.

Cont. 1. 9 o. $* * * 3$ 


\section{Classis et Urdo:}

\section{CRYTTOGAMTA FUNGT (UTRICULOSA).}

Ex minutis-imis futgilli gregatim ligno putrido inuascentes

Tubulis tenuissimis membranaceis cyline dricis intus cavis siruiles, ore ab initio clanso, dein dilatato olinso reroluto. Color albus, aetate oboletus. Sulustantia tenera sicca fragilis, tamen persistens.

\section{SOIENIA OCHXACEA.}

\section{Classis et Ordo:}

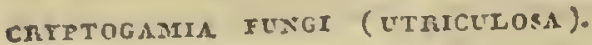

Praecedentem magnitudine et crassitie par. Jo stuperans, emortuorum truncornm cortici in. spersa. Gregarie vel confertim nasci solent

Tubuli rarius crecti, frepuenter inclinati, vel quasi imbricati; ex ovata basi cylindricam formam assumunt, ctians obconicim; extcrue pilis adpressis rigidis ochracei coloris hirsuti, in obtu:tum apicem coëunt. Interne pallidiores glaberrimi nitentes, cavi. Substantia uniformis, sicca frapilis, Humectata mollis fmugosa. IVtagnitudiue diversa nccurrunt, subjecto non raro pulverulento temui cincrascente fibroso strato, una cum tubulis persistcutc. 

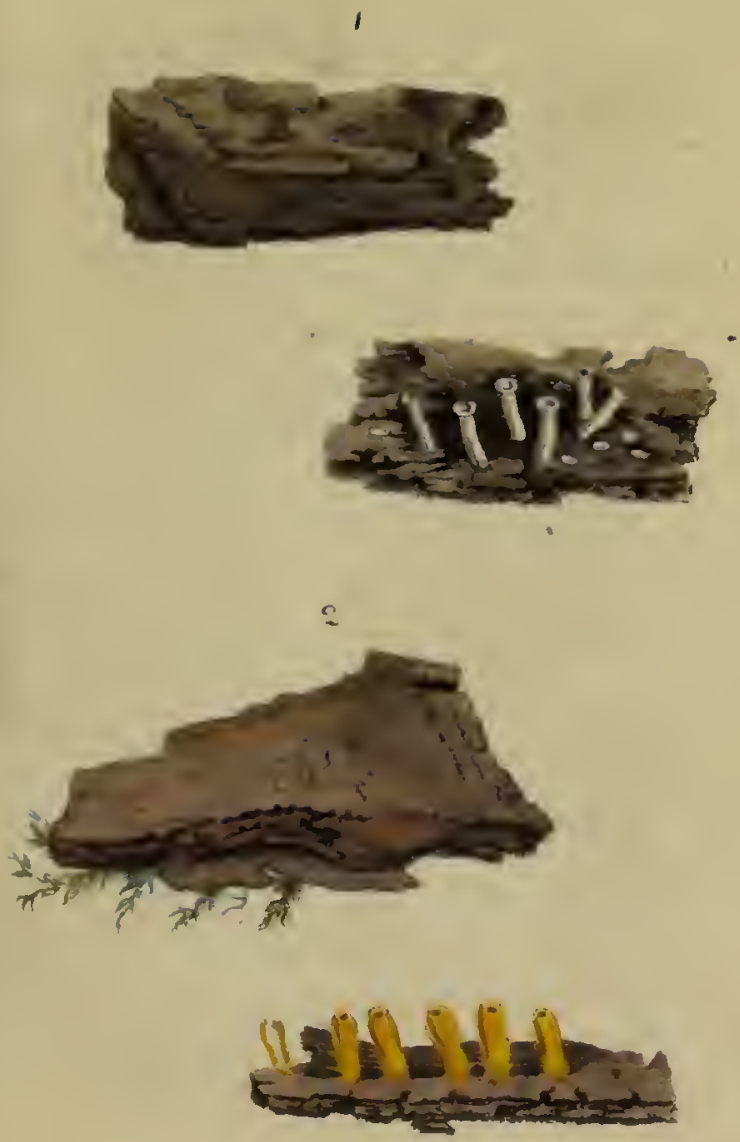

folener candeda. S. Solenia oclrucece. 

A VCTORES

seculi XVIII.

VERZEICIINIS DER BOTANISCHEN SCH IITTEN des 18 lahrhunderts.

Notter, F. P. Flora rustica - with descr. by Th. Martyn. 2. vol. 3. Lond. 92.

Oeder, G. C. Elcmenta bot. 2. P. 3. Hafi. 6., 66. (Edit. gcrm. Kopeuh. 66.)

Ocstreichs Flora. 2. B. 8. Wien, 94.

Olivi, G. Delle conferve irritab. (Mem. della Soc. ital. T. 6. Vcronl. 92.)

Onomatol. Botan. 10. vol. 8. Frankf. 77 .

Ortega, G. d. Curso elementar di Eotanica. 8. . Miatr. 35.

Oskamp. T. T. Tab. plant. terminol fol. I. B. $9 \mathbf{3}$. Pallas, P. S. Reisen durch verschied. Prov, des russ. Reichs. 3. Th. 4. Pet. 76. (5. Th. 8. Frankf. 78.)

Palau, Ant.,y Veril. Explicat. de la Philosoph.

y Fund. botan. de Lin. Matr. 78. Parle pract. 34. 5. vol. 8.

Panzcr, G. W. F. Vers. cin. nat. Gesch. der Lamb. mad Lebeimoose. 3. Nïrnb. 8\%.

Paulet, I. I. Tabula plintar. fungos. 4. Par. nt. Persoon, C. H. Nicier Versuch eincr'syst. Eintheil. der Scliwämmc (N. IVag. für dic Bor. 1. B. 9\%.)

Pilleri ct Mitterlash. Itor per Poscgan. Sclavon. 4. Hud. 85.

Planer, I. I. Index plaut. in agro Erfurt. sp. provent. 8. Goth. 83 .

Rlaz, A. G. Progr. de natura plant. municntc. 4. Lips. 61.

Plenk, I. I. Physiolng. et Pathologia plantar: is Vie11. 94.

Pohl, I. E. animadv. in struct. ac fig. foliormm 4. Lips. 71 。 
SCTEROTIUAI AEGEITTA.

Classis et Orto:

\section{ERYPTOGAMLA TENG (HTMENOCARPA).}

Fungilli ligno emortuo ut farina dense in. herati, accuratils et per icntem inspecti refe. nont grana panici magnindine et forma; globosae nempe sumt vel clliptirae, magis tamen romundae fignrac. Sulstantia solida, tamen fria. bilis, ad vitra angentia intus ex roumdis seminifornibus corpusculis murbinatis, ut in Sclero. tiis pellncidis intra aquam, composita. Color lacteus vel quasi rotulanum ex saccharo confe. ctarum, demun obsoletc albidus. Persistust et coluacicit.

\section{DIIERMA CONTORTUM.}

\section{Classis et Ordo:}

- coyptogamia funge (hymenocarpa).

Muscortum folis inhacrent rotulae candidis. simae compressiac rotundac vel osatac, in sulcos contortac medio in unbilicum mum alte. numve depressite, cortice duplici insmetac. Exterior nempe fragilis crassior, alla, interior obsolcte favescens temior incmliranacea pellu. cons, nigrum continens pulverem (ad leutem ex globosis Besiminibus compositum) filis intertexils.

I) I DE MA OCIIRACEUM:

Iisdcm cum praccedente locis, apicibus fo. fionum sacpe per medium ducris; globosum; ochraceum exterius et membranaceum, iuterne membraua teunior et pallidior ingulosa, pulve. re niyro compacto repleta, ad lemtem priori non dissimili. 

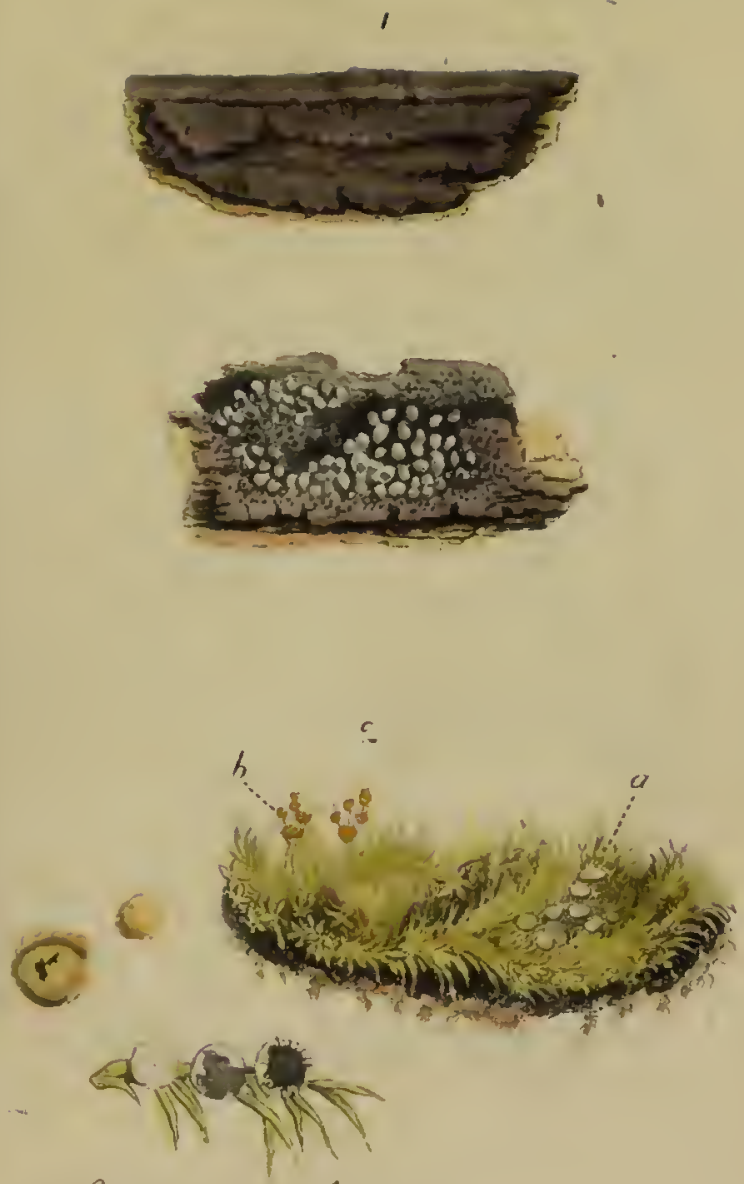

1. Sclenotiume tegerita.

: a Diderma conlortum. h whraceum. 

AVCTORES HRTANICI

\section{seculi XVIII.}

VEFZEICHIISDER BOTANISCHEN

S C II RIT TE N

des 18 Iuhrhunderts.

Desfontaines, R. Plantae rar. (Act. de la Socié: té d Hist. Nat. de l'ar. fol. a Par. 9:.)

Dickson, I. Fasc. planr. crypt. Itritan. 3. F. 4. Lond. 93. (Znrich. 8. 94.)

Dietrich, C. F. Phanzenreich. 2. Th. 8. Erf. 70. Dillenius, I. I. Hort. Elthamensis. fol. Lond. 32. (63.) (Lingd. 74.)

Donavert, E. Instruct. for Collect: var. Subj: of Nat. hist. 8. Lond. 94.

Dörrien, C. H. Verz. oran. $112 \int_{s}$. Gev. 9. 77. Dryander, Ion. Obs. on the Gen. Pegonia ('Trans. of the Linnean. Soc. vol. 1. 4. Lond. 91.) Du Hamel, du Mouçeau la Physique des Ärbres. 2. P. 4. 68 . (Germ. Niirnb. 64.)

Dn Roi, I. P. Observat. botan. 4. Helmst. 7x. East, Hint. Hortus Eastensis. 4. Kingst. 92. Ebermaier, I. C. Vergl. Beschr. derj. Phanzen, welche in den Apoth. mit einander verwechelt werden. 8. Bramnschw. 94.

Edvard's, select collection of one hundred plates, cons. of exotic and british Flowers. fol. Lónd. 75 .

Ehrhart, Fr. Plantac cryptogamae Lin. (exsicc.) Dec. 53. โol. Hamnov. 85 - 95 .

Ellis's, Directions for bringiny Seeds and Plants from distant Comts. P.II.

Framann, C. G. Einige botan. Beobacht. (bor. Anal. ESt.)

Eschenbach, I. F. Ep. de Nectarior. ustı. Lips. 76.

E:march, G. P. C. Beschr. der Gräser - in deni Herz. Schlesw. u. Holst. 8. Leipz. 94. 


\section{Classis et Ordo:}

\section{CIYPTOGAMIA HEPATICAE (IADICALIA).}

Radiculne succosae et teuerac, pellucidae ac albae, utplurimum simplices descendunt, quae medium et praescrion norvum tenent, quibusque humo leviter inhaerent

Frondes prima aetate valde minutae et sim. plices, margine undosac, magis ampliatac sim. plices vel li-tri-quadrifidac et per dichotomiam suludivisae, basi saepe comtutac et in omnem? plagain rosaceae vel gregatim expansae (a), marginte rotmulis segmentis vario undoso quasi $\mathrm{ex}$ cito (b), latimdine variabili. Dantur specimina angustioribus et longioribus, vel obtnsis law. tioribus frondibus (in his tubuli etiam breviores). Nervus per simplicem vel ramosam frou. dem ductus, reliqua teuera et pellucida vireutes substantia albidior.

Flores inasculi granula (Antherae Schmid. Hedw.) prope marginem frondis sparsa, immersa, cuticula tecta, pellucida, vircscentía (hici objecta aeruginosa), demum puuctorum nigrorum iustar in conspectum prodeuut; interne gra.. mulosa massa repleta (c).

Flares feminei tubercula (Germina Schmid. Hedw.) in medio disco versus extrema frordis vel rami sita, inverse ovata, introrsum (qua parte radicem plantulae spectant) nempe arciiora et depressiora, extrorsum sensim latescumt suporne et inferule prominent (d. e.) Nervus ad apicem tuberculi inlcriorem pertingit unicus, ex communi trunco in tot ramos discerptus, quod tubercula frous gerere solet. Vertex tuberculi erigitur conicac formae (tune temporis iusides 

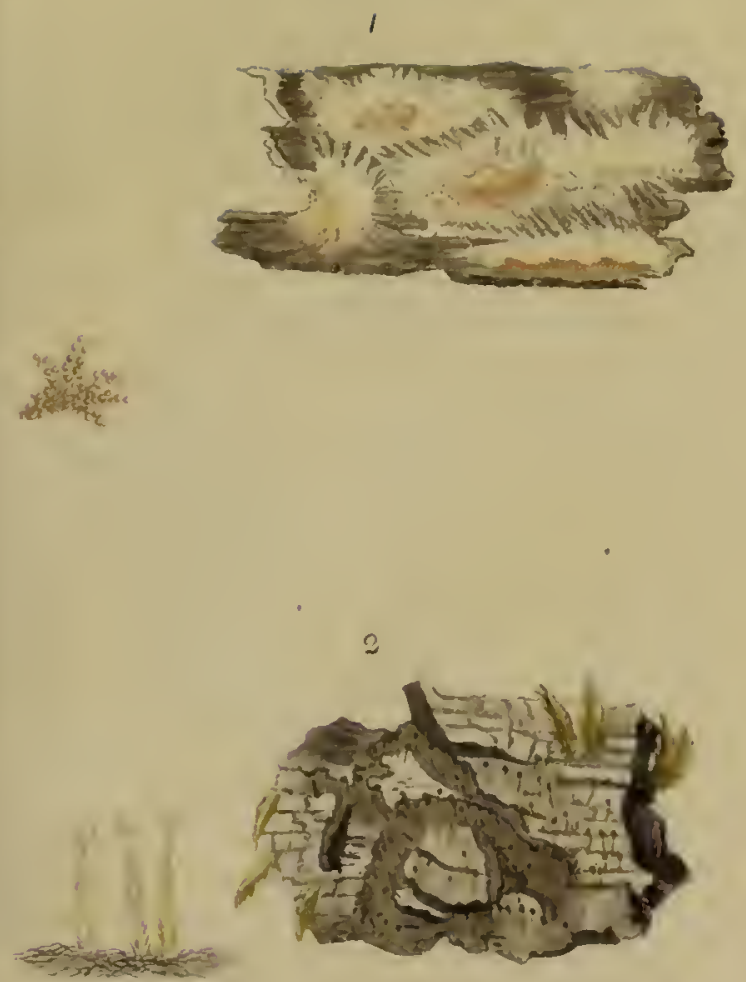

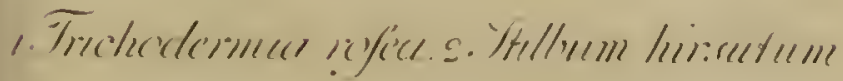



A V C TORES TOTANICI

seculi XVIII.

VERZEICIINIS DER BOTANISCHEN SCII RIT TEN

des 18 Ialihunderts.

Evelyn's silva et terra - with notes by Dr. Hunter 2. vol. 8. 36 .

Fibig, I. Einl. in dic Natnrgesch. dez Pflanzenrcichs. 8. Mainz, 9t.

Fabregou, Plantes anx envirous de Paris. 6tom. 8. Par. 40 .

Fischer, I. B. Versuch einer Naturgesch. von Livland. 9. Königsb. 91.

Forster, G. Florula Insularum austr. 8. Gott. 86. - - I. R. Enclirid. Ilist. nat. inserv. 8. Hal. 38.

Forsyth, will. On the Discascs of Trees and micthod of curc. 3. Lond. 92. (ïber dic Krankh. - der Bäume. Aus dem Engrl. vou 9 . Forster. 8. Mainz, 92.

Gacrtuer, I. De fructibus et scmin. plant. 2 tom. 4. Stutty. 91 .

Gatcrean, Description des plantes qui croiss. anx envirous de Montanban. 8. Mont. 90.

Gefsuer, I. De lianmuc. bellidifloro. 4. Tig. 53. Gemus, S. I. v. Verhand. over de inlandsche Plantecwassen. 8. Haarl. 89.

Gilibert, E. I. Exercitia phytologica. 2. vol. 8. Lugd. 92 .

Gisckc, R. D. Car. a Linné praelect. in ordin. nat. plant. 8. Hamb. 92.

Gleditsch, I. G. Systcma plantar. a stam. situ. Gleichen, IV. Fr. Nenestrs ans dem Reiche der Pf. fol. Nïrml). 6\%.

Gmelin, I. Fr. Car. a Linmé Syst. Nat. Ed. 13.

(Tom. 2. part. . regil. reget.) 8. Lips. 91.

- - K. C. Catal. pl. hor. bot. Carolsrulı. 3.91. ** 2 
tenerrimus stylus, caducus, fuscelli coloris Herlu.) accedcus, sensim vero cylindricus (c) ubacformis $(f)$ demum mutatus in

Capsularn oratam obliquam, milocula. rem, corollatan, milo trumcato apiceque hiall-. te, ex quo corpuscula

Semina (Schmid. IItedw.) acervatim pro. pullulaut et disperguutur (d e) compressiuscula lutescentia, in froude mascula saepe vivipara; cadente ulime et consumto mbo. 
.T. 11.

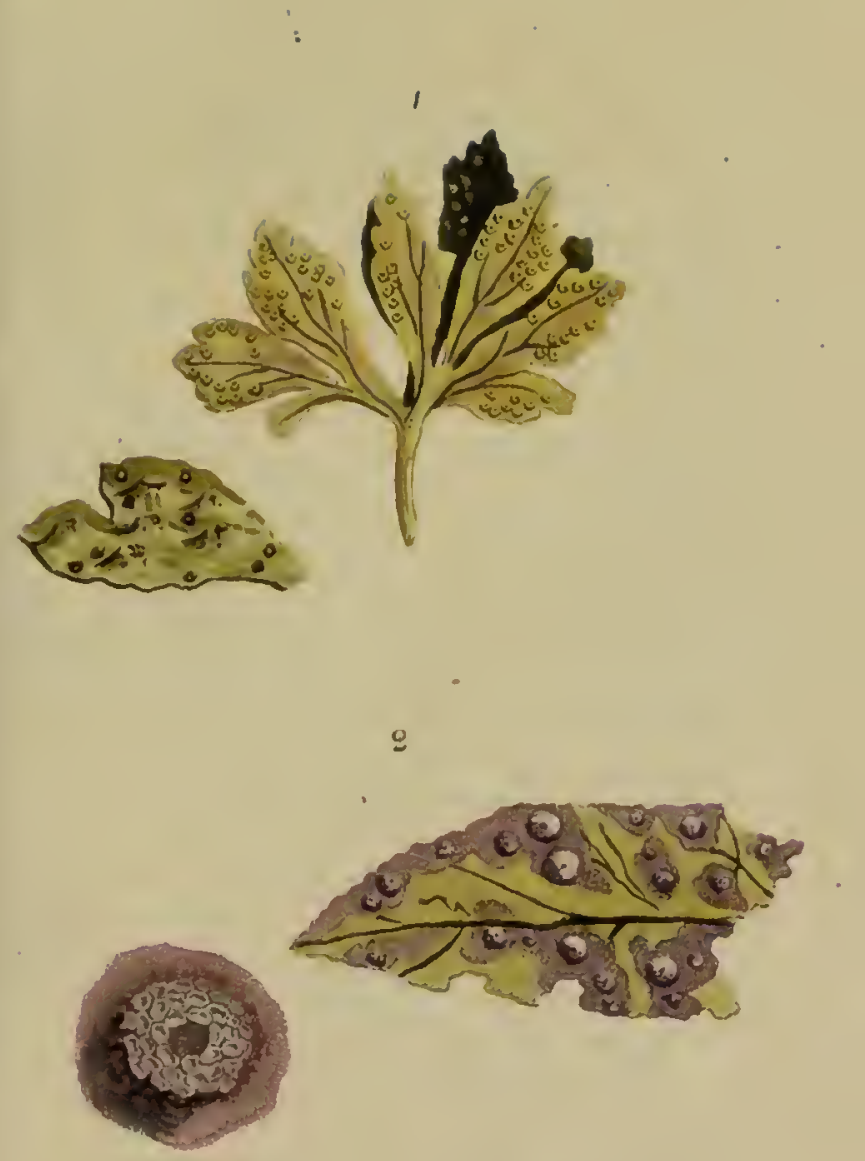

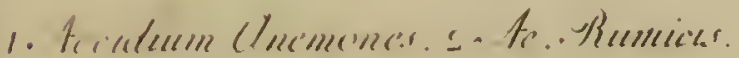



VE Z EICHNIS DEH BOTAIVISCHEN S C H I I F T E N

des 18 Ialirhunderts.

Gmelin, P. Fr. Otia botanica. 4. Tub. 60. Gorter, D. v. Flora Ingrica. 8. Petrop. 61. Bonan, A. Flora monspel. 8. Lugd. 65. Graefer, I. A. descriptive Catal. of 1100 herb. plauts. 8. Lond. 91.

Guettard, I. St. De Pilis et Gland. (Mem. de 1 Acad. de Par. $45-66$. Von den Nebene gefifsen der Pfl. Schrank. 8. Halle, 94.)

Greenivay, I. An Accoiut of the Cassia Cha: maecrista (Trans. of the amer. Soc. v. 3. 4. Philadelp. $9^{\vec{j}}$.)

Flacquet's, Neneste physikal. polit. Reisen durch dic dacisclı. nud sarmat. Karpatl. S. Nürnb. 9o: Haenke, F. Limei Gen. plant. 2. vol. 8. Vien: 91.

Hagen, C. G. De Ranunculis pruss. 4. Reg. 8\%. Hales's, St. Statical Essnys. 2. vol. 8. Lond. 69: Halle, I. S. Deutsche Giftpl. 3. Berl. 8\%.

Haller, A. v. Biblioth. botan. 2. vol. 4. Tiguri

Happe, A. F. Flora crypt. depicta. 4. Dec. fol. Berol. \&if.

Hanbury's, complete Body of plant. and garde. ling. 2. vol. fol. Lond. 70 .

Hedwig, I. Theoria gen. et fructif. plant. crypt. 4. Petrop. 84.

Hellenins, C. $N$. Spec. Calendar. Florae et Fau. nae Aboünsis. 4. Ab. 86.

Flenitier, 1., C. L. Geraniologia. fol. Par. 88. Hill's, I. History of plants. fol. Lond. 51. Holmskiold, Theod. Beata ruris otia, s. Cory. phaci Clavar. Ramariasque complect. Fol. Havn. Honann, C. De Monarda. 4. Traj. ad Viadr. gr. 


\section{Classis et Ordo:}

CRYTTOCAMIA HEPATICAE ( METTTIVALVIA).

Radiculce munc:osac fibrosae deusae c nervo quasi medio oriundae, quibus humo tenaciter adhacrent

Frondes in longum porrectac, cxtrcmitate latiores subrotisudac, margine simato - lobatac, zuedio depressac, lacves, pellucidac, lacte vi resceiltes.

AT(x.sculi Rores (Antherac Hedu.) tubercil. la sessilia in frondis depressiori disco, congesta xumerosa atroviridia (a) vel lutescentia (li), e quibus (transversim una cun substantia froudis sectis, m) massam gramulosan, per vesicnlosam opus cuticulae scatnricutem (d) obscrvavit Hed. migius.

Teminei Rores ab cxtrema frondis prima. riae, aut lobi lateralis squammla (calycina) coll. cava laciniata (b. c), conimula contimno frou. dis vegetationc intra ipsins frondis discum ap. parente (c) originem dncunt. Machinmlac pistilliformes (f) intra hauc squamulam, de quibus mua cxsurgit calyptrata, stylo cornuata in vertice, adhaercute de latere illis machinulis, junior globulis hirsuta (g) deiı glabra (h), maturitate rumpenda, turbmata quadridentata (i).

Pedumeulus (Scta) 2.3 pollicaris, albissini pellucentis coloris, crectus, celeri elongatione

Crojsulam clevat globosam (i) fuscam, in 4 valvulas longitndinaliter dehiscentem, aequales patentes, demum reflexas.

Semina plurima, ovata (1) adhacrentia filis longis, exheljcibns duabus, circa commu. nem axiu circumvolutis, clasticis, in valvnla. rum ccutro demun barbulac confusae instar Eurrectis. 

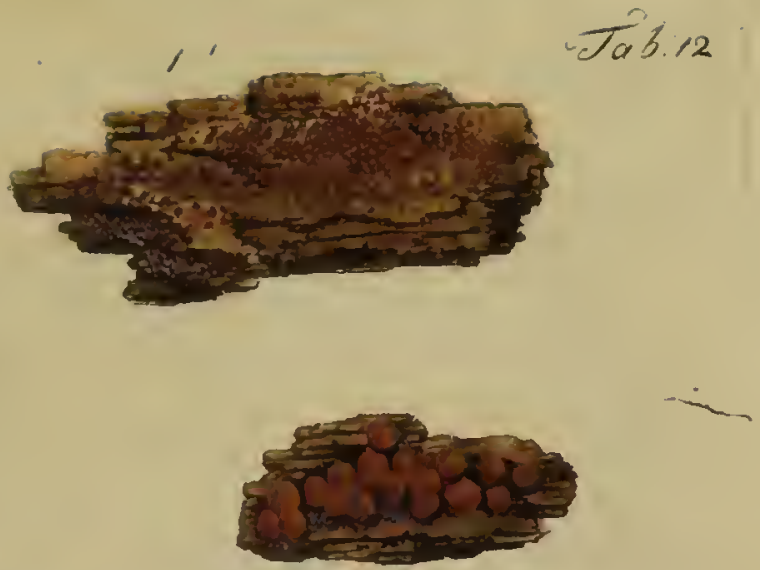

2
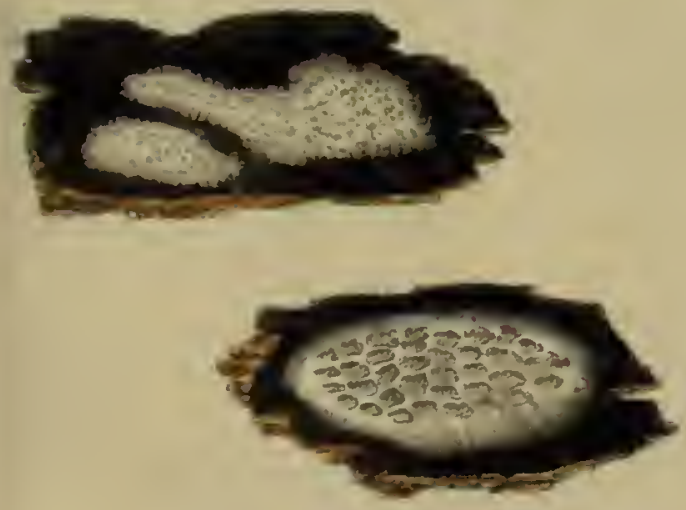

- Sincerice menuata. 2 Mucilago reticulatis. 



\section{A VCTORES BOTANICI}

seculi XVIII.

VERIEICHNIS DER BOTANISCHEN S C M R I F TEN

des 18 Iahrhunderts.

Honckeny, G. A. Synops. plantar. Germ. Tom. r. 8. Berol. 92. (c. Car. L. Wilidenovv).

Hope, T. C. De plantar. mot. et vita. 8. Edine burg. 87 .

Hoppe, D. H. Botanisclies Tischenb. 8. Regensh. Host, $\mathbb{N}$. (Jacq. collect. 4. Vien. \$8.) Honstoun, G. Reliu. plaut. 8. (ed. germ.) Now rimb. 94 .

Humboldt, F. $\Lambda$. ab, Florac friberg. specimen. 4. Berol. 93. (Aphorismeu ïbers. von $\mathrm{B}$.

Fischer. 8. Lcipz. 94.)

Jacquin, N. I. V. Oxalis. 4. Vien. 94.

- - I. F. a, Tria gen. plant. nova. (nov. act. liclves. v. .1. 4. Basil. 87.)

Jefferson, Th. An accomit of the Sugar Maple Trec. 8. Philidelph. 92.

Jundzilla, B. S. Opisanic roslin iv Prowincyi

Wiclkiego Xiestwa Litewikicgo naturahuie

(Beschr. der in Grofsh. Lithanen wild. waclis. Pfl. ) 8. Warsch. 91.

Iunghans, P. C. Icon. plant. offic. Cent. 1. Fol, Hal. Sal. 92 .

Jussieu, Ant. Ianu. Genera plantarum. Recu. di curav. notisque anx. P. Usteri. 8. Tnr. 91. Keruer, I. S. Giftigye und efsbare Schwämme. 8. Stuttg. 86.

Klipstein, I. C. G. De Nectaries plant. 4. Ien. 74Kuorr, G. W. Hegll. Florac v. Thes. r. herb. 2. Tom. fol. Nürnb. 66 .

Koelreuter, 1. G. Das cntdeckte Gehcimu. der Cryprogamic. 8. Carlsr. $77^{\circ}$

Külpir, A. B. Comment. de sıylo. 3 . Gryph. 3马. 


\section{A I TIOCEROS PUNCTATUS. Classis et Orslo:}

CIPPTOGAMIA HEPATICAF (RADICALIA).

Rradiculae ex basi descendunt satis longae simplices vel parum ramosale (d f), passin ctiam ex trunci frondosi (d) lacere, tenerrimac, pellu. cidac, virescentes autequam terram atumgant; cui adhaereut

Frondes ex andrustiori basi in orbem ex. pansae, disco concavo, margine eleratac (E) undoso plicatue, laciulatae: lacinulis simutis, cre. mulatis, quasi crispac. Frequenter laciunlac ali. quine vario modo incisae super ipsum discun adsurgtut (d) quasi viviparac (a) vel ex ipsa basi prodeume. Ubi gregatinı foondes rescume in. terdum grariliores aduascumenr ita profunde (ex obscrvatiouc schmidelii) scctac, ut pimatan formain Antzoc. multifidi, masis mimusre regu. larem aut compositam, tamell semper turbiua* taln, bene exprimant.

LFencirit flores vernuculac in discn frondis clevatae sparsac, apice in lacinias fissac, calyciformes, recoudunt globulos (Autheras Hudw.) nbovaios, crocei intensi coloris, vesiculares mollcs, et perlucentes, apice reorsum calyci iuhac. rentes (b c d). Post aliquam horum adparitioncm

ficminet fores in cadem planta, nt coruicula s. voscula per hiatum corticalis substautiae dilitatae (Cinlyx monoplyylus, cylindricus trum. catus) (g) in conspectun veninut; ex oblonga forma scisisn in teretem mutuntur, vertice ca. Iyptra et brevi stylo, crocco colore tiucto co. roula (li), succrescunt denique cornicula (it) in Cripusulcum subulatam (Maplidia Neck.) sc. mibivalvem (f), vel bivalvem: valvulis exterue convexis (g), varie flcxam. Colmmella (disscpimentum) libera a basi ad apicem extensa, cti adhacreut

ieminr numerosa (h) glohosa, extus te. nerrimo reticulo exarata et quasi tuberculata, matura nigerriuna solida, filis ex fusco croceis: e duobus helicibus contortis, clasticis columel. lac adlixa (i). 



\section{AVETOFES HOTAITCI}

seculi XVIII.

VERZEICIINIS DER BOTARISCIIEIV S CII I I TEN

des 18 Iahrizunderts.

Trew, C. I. Cedrorum Libani hist. 4. Norimb. 57.

Turra, Farsetia novum geuus 4. Veuct. 65.

Uslar, I. von, Fraymente neuerer Planzcukunde. 8. Braumsciliw. 94.

Usteri, P. Anualen der Botanik 12. St. 8. Zü. rich, 92.91 .

Vahl, M. Symb. botiu. 3. P. fol. Havn. 90-94. Vaillant, S. Sermo de structura florum. 4. L. B. 48 .

Vandelli, D. Viridar. Úrisley lusit. 8. Olisip. 89.

Vellozo, Ios. Flora fuminensis. 6. vol. fol. Lisb. (inedita).

Ventenat, snr les pirties des Mousses qui ont eté regardécs comme fleurs males ou fleurs femelle. (Journ. d'hist. natur. T. 1. 4. Pa. ris. 92.)

Viborg, E. Forsög til syst. Dimske Navne af indenlandske Plauter. 9. Kopenh. 93 .

Villars, Hist. des Plantes de Dauph. 4. T. 8. Gratianop. 87.

Vitman, Fulg. Summa plautarum - 6. T. 8. Me. diol. 8992.

Vogel, B. C. Supplem. plaut. select. 2. Dec. fol. 92.

Wachendorf Hort. nItraject. 8. Tr. ad Rh. 47. Walther, F. L. Theoret. pract. Handbuch des Naturgesch. der Holzarten. 8. Bayr. 95. Weber, G. H. Supplem. Flor. holsat. 8. Kilou. 87.

Weigel, C. E. Flora pomerano - rugica. 8. Be. rol. 69 .

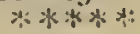


A priori differt: comvexis limris, oblongis, simutis, angulo:is, divaricatis, confucutibre, compactis, ope lentis obovatis

Lomentis vel pyriformibus ; Intra scptula transversa pellucent granula demum prorumpentia.

\section{PEZIZA IENTICUIARIS.}

\section{Classis et Ordo:}

\section{CRYTIOGAMTA FUNGI (UTRICULOSA).}

Dematio insidebat nitida ac durabilis plan. tula brevi stipite instructa, qui sensim dilutaur iu

Crrpulam seu potius Acetabulum modice cavum, cliam disco convexo vel plano instru. ctum, margiustum. Color ex terro luteus. Substantia caruosal fibrosa, intra quam ad lew., is augmentun apparent

Llisculi e basi angusta cyliudrici, temuis. simi c pellucida alba membrana pro more lu. jus ordinis contincut

Besimiza 8.10, contigna minutissima, fellucida, granulosa. 


\section{A VCTORES BOTA NICI}

\section{seculi XVIII.}

VERZEICHNIS DER BOTANISCHEN S C II R I T E N

\section{des 18 Ialriunderts.}

Weinmanu, I. Tract. de Chara Caesaris. Carlsruh. 8. 69 .

Weis, F. W. Vorbercit. zum Unter. in den Grundk. der Bot. 4. Gött. 31.

Weizenbeck, G.A. Bot. Wuterh. 2. I. 8. Millich. 85.

Wendland, I. C. Ericarum icones - 1. F. 4. Tur. 94.

Wernischeck, I. Genera plantar. 8. Vieu. 64. Wilcke, S. G. Hort. Gryph. 8. 65. Willdenow, C. L. Pliytographia. F. ג. fol. Erl.

Willemet, Monograplic - de la Fam. des plant. étoilecs. 8. Strasb. g1.

Willich, C. L. Observat. bot. 8. Gott. 62.

Winterl, I. I. Index horti botall. Univ. Inun. gar. qluae Pestini cst. 8.88.

Withering, IV, aud stokes, I. Bot. Arrang. of British Plants. Ed. 2. 3. vol. 8. 92.

Woodward, $T$. I. The hist. of a new spec. of Fuchs (Lin. Truns. v, r.)

IVoodwille, W. Medical Botany. 3. v. 4. Lond. 92.

Wolf, N. IM. de, Gen, et Spec Plant. vocab. charact definjta. 8. in Nitarienwerd. 81.

Wolff, I. P. De filicum seminibus Ien. 70. (Lud. wig delect. opusc, ad sc. 1tat. : pect. v. 1. 8. 90.)

Wright, IV. Au account of the Med. Pl. gro. wing in Ianaica (Lond. nedic. Iourı. v. 3. P. III.)

Wulfen, F. $x . a$, plantac rar. Carinth. (Lacq. Collect. 4. vol. 4. Vind. $36-90$ ) 


\section{AVCTORES BOTANICI seculi $X V I I I$.}

VERZEICHNIS DEIT BOTANISCHEN SCH H I T T E N

des 18 Iahrhunderts.

Wünsch, C. Z. Briefw. über die Nat. producta. Xuarcz, G. et L. Gily, Osservazioni fitolog. Zannichelli, G. G. Istoria della Piante di Lidi. Zinu, I. G. Observ. hot. et aulat. (Com. Gort. Zorn, I. Americ. Geväclise (Iacq. select stirp. Zumeric.) 3. Cent. 8. Nimrnb. 88. Zuccayni, Att. Dissertazione - di una pianta pannizz. dell" Abiss. 8. Firenze, 75 . 

DEUTSCHLANDS FLORA. F 
- Cum rerum Natura nuนquam magis quas in miximis tota sit. PLIN. 


\section{VO R B E I I C II T.}

E: gereicht dem Forschungsgeist unseres Zeitalters zur Ehre, dafs man angefangen hat. die sonst wohl für überflinssig, oder doch für minder wichtig angesehenen kleinen Vegetabilien "), vielche im gemeinten Leben unter der Beneunury von Moofs, Schwamm, Schimmel bekannt sind, im linneischen Pllanzensystem, als Servi, Vernaculi, Nomades - die lezte, 2fte Klasse einnehmen - dafs man angefanger hat, diese sorgfaltiger, und an ilunen derselben mannigfaltigen, zun Theil mit gröfsern Gewächsen übereinstimmenden, zum Theil auch sehr davou verschiedeucn innern und äufsern Bau zu beobachreu. Nothwendig muste anch die Anzahl der Arten zu gleicher Zeit dadurch rermehrt werden. Nur für Deutschland berechnet, kanu man gegenwärtig verborgenblï. hende Gewachse (Cryprogama), wo uicht mehrere, doch gewifs eben so vicle annelimen, als

$$
x=\text { siche:- }
$$

") Muscos et qui IMuscas legit, huic aliud non suppetit uegotium. - Projecta vilior Alga. 
sichtbarblihende (phaenogama). Auch mich be. lelurte unter der Ausarbeitung dieses Taschen. buchs, die bestindig zunehnende, noch lange nicht erschöpfte Anzahl, von der Unmöglich. keit, alle diese, nach und nach mir bekannge. wordene Cryptogamisten, bequem is cincm Jahrgange unterzubringen. - Ich ïberliefere also hier dem botanischen Publicum vorlintig cine Abtheilumg derselben; die vier sogenannteu erstern linneischen Ordmuggen, oder viel. mehr Familien: Miscellanere, Iilices, Musci, Hepaticae. Eine diesen ähnliche Familic, besser, natürliche Orduung, sezte ich zu : die li. chenes. Aufserdem versuchte ich mach den Standort, der äulsern Form, der Umhiullum, der Vermehrungs - und Fruclittheile, oder was man zur Zeit sonst dafïr aunehmen kamn, Ord. mumgen, welche mur allein daranf begrüudet sind, zu crricliten, deren lienenumng und aus. führliche Erklärung die zumächstfolgenden Blit. ter iu der Kunstsprache enthalten. Zngleich fauls ich hier bemerken, dafs cinige (den Kn。 piern 2. G. gegenüberstehende) Aufschriften der Ordunngen, welche sich auf eine friblere, aber nicht beibehaltcue Eintheihug besiehen, nach diesen abzuändern sind.

In der Wahl der leichtern von sichtbaren zufcern Theilen hergenommenen Charactere, so 
wie der Ordnungsfolge der Gattungen, glanbe ich kaum bei der guten Absicht, die Kenumirs Manchem dadurch zu erleichtern - Entschuldigung zu bedürfen. Denn auch für diejenigen, rvelche eine schärfere und mülsamere tntersuchung der verborgenen Theile, oder cine grüssere An. zahl von Gattungsnahmen, zumalilen bei den Laubmoosen, nicht sclienen, sind die Hedrvi. gischen Charactere derselbeu, nach der achter Ausgabe der Linneischen Gen. I'l. mitgetheilt worden. Ich beluzte verschiedene zu Unterabtleilungen bej deu Lanbmoosen; in der Uebera zeugung, dafs es Anfangs leichter ist, Arten nuter wenigen schon bckannen, aber schärfer bestimmten und abgetheilten Gatmigen aufzu. finden, als ncue Gatumgnahmen und Chara.. ctere sich bekaunt z.11 maclıen. Die zahlreiche Fasuilic der Flechten, welche beinahe alle vorhergehende zusaminengenommen an Menge der Arten und Halbarten ïbertrift, suchte jch deswegen auf so wenige Gattungen zurükzuführen, als es nur immer die grofse Mannigfaltigkeit jh. rer äufsern Gestalt erlanben wolle. So wird man 2. B. unter Lobaria, Verrucaria, verschiedene meiner elmaligen Gatungen in Unterabtheihu. gen verwandelt findeı, $1 \mathrm{~m}$ dem Ungeïbeul das Aufsuchen der Arten zu erleichtern, oder docis. durch minder auffallende Gatuugscharactere uicht zu erschweren. Vicle Arten und Gatmu. $\varepsilon$ $x^{3}$ gell, 
sen, melshe man bisher nuter die Flechten versrzte, wie Lich. polyphyllus Wulf., mruina. tus, ericctorum, capritatus - Enducrerpon, Opegrapha u. a. musten nach denen zur Grundlage angenommenen Kenuzciclicu der Ord. nung, dayon getrennt uud in andere Ordnun. geu vervieseu verdeu.

Das Verzeichuifs der Arten ist zugleich cine getrene Augabe von dem, was ich vor mir hatle nud was ich mit Ueluerzengung anfnchmen zu kömucu glaubic. Die nach einer differentia specifi. ca geseztch Punkte zeigch anf cine dauit vorgenommene Veräuderung. lis folgeu daranf die Nahmen dex nachgeseheneu nud mit meincu Excmplaren verglichenen Schriften, die Anzeige des mir bekannten oder mitgetheilten stand. orts, bei seltuen Arten, und in den Anmerknn. gell Einiges zur Berichtigung oder nälıen Kenntuifs derselben.

Der vorgesezte Asterisk deutet auf Arten, weiche um Güntingen oder anf dem Harzgebir. ge zu linden sind. Varictäten, Halbarten (sub. species), anch Arten, welche ich als solche aufzufuhren noch nuentschlofsen war, findet man entweder in Klammern ( ) den Anmerkun. gen, oder olnte Bezifferung der nächstver. vandien Ars beigestellt. 
Alles n̈brige, sowohl in Bezielnmg anf Schrif. ten - und Scliriftstellerverzeichnifs, als anf die Kupfer und ihre Beschreibung it $t$ bo wic bej dem ersten Jalirgang dieses Taschenbucls. Hier muste noch ein vollstäudiges Regisıer angeliängt werden, zur Erleichteruug der öfters sehr ver. schiedenen Bencmungen einer und derselben Art, oder zur Rükweisıug der Verscliedenleit melırerer gleichmahmiger Arten.

Anfangs bin ich Willens, gevesen, dem VTexkchen cine ausfillrliche Erzällung aller anfeinander folgenden Entdeckungen in dieser Classe und ihre Würdigung vorzusetzen. Al. Jein ich sahe mich genötligct aus Vingel an Zeit - aucl in der Erwartung Dlanches in der Folge vollstündiget sagen zu kümen - melire. re Nachträgt nebst einer bereits fertigen Carpo. logisclien Tafel für eineu küuftigen Jahrgang zu verspareu. Eine Verzögcrung voll der Art, hoffe ich, wird meiner Absicht in so fern zum Vortheil gereichen, da wahrscheinlich bis dahin nenere Eutdeckungen sich an die Reihe der frü. hern werden auschliefren und vieles deuticher erkennen lassen, was gegenwärtig noch als un. bekanntes Land in Dunkelleit verlüillt ist.

Es ist noch ein grolser Theil zariick und zuch noch Raum genug übrig zur Belchrung, dis 
die ich mit Dank von Kunst - und Naturge. rieihten annehmen und mich dadurch mehr oder weniger berufen fulllen werde, anf diesem noch ungebalnten Tiege fortmwandelu und ihn ron Hinderaissen zn rcisigen, oder es an. dern zn Z̈bcrlasscn, einen bessern VVcg, zux Kennulifs cryptogamischer Gewächse, auszu. mitteln.

$$
\begin{gathered}
\text { Güttingen, im Lenzmonat } \\
2796 .
\end{gathered}
$$

\section{G. F. $H$.}

\section{Nachsclirift.}

Der jïngsten Acusscrung des Hns. Verf. ge. mäls, sollen vou Zcit zu Zeit in klcinen Nach. triger die Znsätze den I icblıbern der lier bcschricbenen Gewäcluse (viclleicht bis Ostern 2797. dic Fortscezmng davon, od(r eine neus Ausgabe des ersten Theils) viberliefert ma zur Bekanutmachung und Mitheilumg ihrer Bcirri. ge alle $\mathrm{nm}$ Dentschlands Flora verdicnte Ge. lẹrte anfgefordert wcrdeu. Dic adjenda et cmendrnda bittct unau gchörigen Oris cinzu: tragen, das Blat gl aber, welches der zurciten Lieferung.bcigedruckt worden, vou der erstern vegzunehmen. 


\section{Classis XXIV. \\ C R Y P T O G A I A.}

- RDXXE\& partibus fructus ratione-situs vel figurce exstructae sequeutes sunt:

1. Radicalia. Finctificationes inter radices vel folia sirae.

1. Marsilea. Fructificat. 2.3. pedicellatae subcompressae e petiolo folii paul. lo supra radicem egredicntes.

2. Salvinia. Frnctificat. 4-9. glomexa: tae inter radices verticillätas.

3. Pilularia. Fructificat. solitariae inter folia et radicem subsessiles.

4. Isoetes. Fructificat. intra basin dilatalam foliorum.

II. peltata. Fructificationes in clavam ovato - oblongam tcrmiualen digestae, verticillatae, stipitatae. Singu. 1. peltata, orbiculato - polygoun, in. ferne adnectens tubos $4 \cdot 7$. stipiti

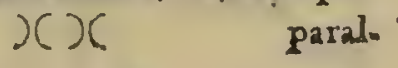


parallelos, apice rotundatos, interne dehiscentes, in quibus grana (Semina Hedw.) plurima subglobosa apiculata, quibus basi adnexa fila quatuni per paria coadnuata, clastica, terminata spathula (Authera Hedw.) pulverulenta.

5. Equisetum. Fructificat. angulatae in. ferne deliscentes $4 \cdot 7$ loculares.

Hace sccundum octavam editionem Gen. pl. Lin. admumetato Lyco. podio andiunt: NISCELLANEAE.

III. AnNurata. Frnctificationes in aver. sa pagina frondum in lineas, pun. cta, in disco vel-margine rel per totam puginam digestae. Squama s. imolucrum e folio enaum varic deliscens, sub quo capsinlac subglubosae stipitatae ammulo articnlato elastí co cinclae, transversin rumpentes emittentes corpnscula seminiformia.

6. Polypodium. Capsulae distributae in puncta subrotunda. 
7. Oroclea. Capsulae distributae sub frondis pinnis pinnulisque recurvato contractis.

8. Pteris. Capsulae distributae in lineam subtus cingentern marginem frondis.

9. Asplenium, Capsulae distributae in lineas rectas, saepe confluentes.

IV. brvarvia. Fructificationes in spicas connatae, in racemos collectae vel in axillis squaururum vel ipsorum foliorum sessiles, Capsulae anuulo destiturae, transversim deliscentes, bivalves.

10. Ophioglossum. Spics articulata : are ticulis transversim dehiscentibus.

11. Osmunda. Spica racemosa; capsulae distinctae subglobosae sessiles vel sub. stipitatae.

12. Lycopodium. Capsulae reniformes in axillis squamarum imbricatim digestarum spiciformium, vel in axillis foliorum sessiles.

$$
x x^{2}
$$
Hre 
CAYPT. CALYTTRATA.

Haec secundum octavam editionem Gen. pl. Lin. excepto Lycopodio audiunt: FIIICES.

V. calyptiata. Fructificationes femineae irirolutae calyptra, stylo coronata, nuda vel pilosa, laxa magna vel coarctata, quae capsulae adolescentis apici adhaeret eamque desuper tegit. Capsula (Theca Hedw.) unilocularis interne a basi ad apicem columella (sporangidio) instructa, pedunculo (setae) insidens, perichaetio, vaginula cincto, in plerisque transversim dehiscens operculata: operculo annulato 8. exannulato; basi interdum intumescens, dilatata in apophysin orbicularem, subglobosam, umbraculiformem; orificio nudo vel obsito epiphragmate, peristomio simplici: den. tibus s. ciliis $4 \cdot 80$, erectis s. refexis, rectis s. tortis capillaribus, triangularibus, lanceolatis s. setaceis, acue tis s. obtusis, integris 8. bifidis, bipartitis s. pertusis, margine lacinulatis 
tis s. crenulatis; vel peristomio composito, duplici: interiori membrana. ceo, r: iculato s. varie laciniato s. e pluribus distinctis dentibus, ciliis cum exterioribus altermantibus, constructo. Semina minuta, alternante contractione vel dilatatione peristomii emissa.

33. Trentepolilia. Partes fructificationis masculae et femineae $n t$ in reliquis muscis. Capsula incognita.

Corpuscula gemmiformia intra axilläs foliorum ovata colorata squanis adnatis tecta.

14. Phascumn. Capsula subsessilis clansa cum rudimento operculi.

15. Buxbaumia. Capsula ovata obliqua

basi rentricosa.

26. Sphagnum. Capsula apophysi orbiculari insidens, saepe calyptrae parte inferiore abrupta basi cincta.

17. Splachnum. Capsula apophysi maximae subglobosae s. umbraculiformi insidens. (Perist. simplex dentibus 8. geminatis.)

$$
x x^{3} \text { 28. Po. }
$$


28. Polytrichum. Capsula interdum apo. physi quadrangulae insidens. Calyptra ex apice deorsum pilosa. (Pcrist. simplex dentibus plusquam jo membranula (epiphragmate) apice connexis.)

19. Orthotrichum. Calyptra sursum piIosa.

20. Encalypta. Calyptra campanulata, magna laxa, glabra.

21. Gymnostonum. Pexistomium nullum.

22. Tetraphis. Peristomium simplex: dentibus 4 pyramidalihus. (Calyptra plicato - striata glabra.)

23. Bryum. Peristomium simplex : ten. tibus s. ciliis plusqum 4; calypua glabra capsulae insidens coarctata; seta terminalis; llus gemmi.rel capituliformis. (Caulis erectus.)

24. Mnium. Peristomium duplex: ex. terius dentibus 16 ; interius membranaceum lecinulatum, pertusum; ca- 
lyptra glabra; flos masculus femineusque teminalis. (Caulis erectus.)

25. IIypum. Peristounii dentes simplici duplicive ordine; Ros masculus femineusque axillaris, gemmaceus. (Caulis frondosus, ramosus.)

26. Fontiualis. Peristomium duplex: extcrius dentibus 16; interius reticula. tum. Capsulae seta hrevis perichae. tio imbricato obroluta.

27. Audreaea. Capsula setae perichaetio imbricato obrolutae, insideus, unilocularis, medio dehiscens 4-valvis: valvulis apice calyptra counexis.

Haec secundum octavam editionem Gen. pl. Lin. exceptis Tientepohlia et Andrenea, andiunt: muscr. quorum genera secundum metho. dum Heduigii sequeutia assumta sunt :

prascur. chps. orata, subsessilis . setae brevis insideus, undique clansa, interdum cum rudimento operculi, nunquem dehiscente. Masc. subdi-

$$
x x^{4}
$$

scoi. 
CYYPT. CAIYYTRATA.

scoides terminalcs, vel gemmacei axillares.

SPHAGNUAT. CAJ's. sessilis in orbiculo, pedunculum teminante; saepe calyptrae parte inferiore abrupta cincta. Periscomium nullum.

Granostomum. cars. ovata. Peristo. mium nullum. MASC. gemmacei in endem, rel discridei iu distincis planta.

овs. G;mnostomun Hedw. Masc. discoidei in distincto individuo.

Hedwigia II. MTasc. gemmucei in eo. dem, axillares.

tetrarars. caps. oblongi. Peristomiun simplex: dentibus quatuor, pyramidalibus.

MAsc. discoidei, terminales, in distincta planta.

octomlephars, caps. ovata. Peristo. mium simplex: dencibus octo, triangularibus.

MASC. 
CRYPT. CAIYPTRATA.

MAsc. subdiscoidei, axillares, in eadern planta.

SPLACHNUMY. CAYS, cylindraces, apophy. si umbraculiformi, 8. subglobose intus carae, insidens. Peristomium simplex : dentibus octo, geminatis.

Masc. gemmaceo - discoideo; pedun. culati solı fertiles.

ormmia. capsura ovata. Peristomium simplex : dentibus sedecim, latiusculis.

MAsc. gemmacei, in eadem vel distincta plauta.

ons. Grimmia H. Perist. dentes re. Rexilos. Masc. axillares genmacei, terminales capitulif. in eadon planta. Weissia II. Perist. dentes integri. Masc. expitulif. terminales in diversa.

Excaryp'a. cays. cylindracea. Peristomium sinplex: dentibus sedecim, angustis. Calyptra campanulata, magna, laxa, glabra.

$$
\text { X) } 5 \text { Mase. }
$$


MASC. genmacei, axillares, in eodem individuo.

Dicnanum. caps. orato - oblonga. Poo ristomium simplex: dentilus sedecim, latiusculis, bifidis.

M.ASC. gemmacei axillares, in eadem s. distincta planta.

OBs. Iissidens II. Masc. A. gemmacei axillares in eadem planea.

Dicranum II. MTasc. capitulif. cermirales in distincia planta.

IRICHOSTOMUn. caps. oblonga. Peri. scomium simplex: dentibus sedecim, bipartitis: laciniis setaceis rectis lon. gis.

MASC. gemmacei axillares.

DIDXMODON. CAYs, oblonga. Peristom. simplex: dentibus 32, filiformibus, per praria approximatis.

Masc. axillares s. terminimales.

TORTULA. GAPs. oblonga s. cylindracea. Perist. simplex: dencilus capillaribus, spiraliter convolutis.

M A s . 
Masc. gemmacei in eoden rel diverso individuo.

obs. Tortula If. Masc. gemmac. in eodem individuo.

Barbula H. Masc. capitulif. in diverso individuo.

weissia. caps. ovata. Peristomium duplex: exterius dentibus sedecim, la. tiusculis: interius dentibus rotidem, latiusculis, erosis; s. nullum. Calyptra conica, pilis erectis.

MASC. gemmacei axillares et terminalcs.

poHLra. caps. otato-oblonga, apophysi obconicae, angustioni insidens. Peerisiomium duplex: exterius dentibus sedccim, latiusculis; interius nem. brana sedecimpartita.

MAsc. gemmacei in distincta planta. funaria. caps. obconica. Paristomium duplex: exterius dentibus sedecim, obliquis, apice colaerentibus; 'interius 
CKYYT. CAIYPTHATA. rius ciliis totiden, membranaceis, planis.

Masc. discoidei in diversa planta.

BryUM. CAPs. orato-oblonga. Peristo. mium ¿uplex: exterius dentibus sede. cim, latiusculis, acutis; interius menbranacenm, plicato - carinatunı, lacio niatum; laciniis latiusculis capillari. busque alternis.

sisc. capitati s. discoidei s. gemmacei in eadem s. diversa planta.

oBs. Bryum II. Masc. capituliformes. Muium H. Masc. discoidei.

Webera H. Pcristomium incerius cum et absque ciliis.

rmara. caps. ovata. Pcristomizm duplex: exterius dentibus sedecim, latiusculis; interius obsolete sulcatum, laciniatum: laciniis subaequalibus, pertusis.

MAsc. gemmacei, pedunculati, axilLapes. 
Mesia. CAps, oblonga. Peristomium duplex: exierius dentibus sedecim, brevibus, obtusis; interius ciliis totidem, acutis, distinctis vel opere reticulato connexis.

MASc. femineis approximati vel discoidei in diversa planta.

BARTrAMIA. CAEs. sphaerica, striata. Peristomium duplex: exterius dentibus sedecim, cuneiformibus; intcrius membranaccum, plicato - carinatum, apice lacinulatum.

irasc. Femineis approximati.

FONTINALIs. CAPs. insidens setae brevi, perichaetio imbricato obrolutac, 'ob. longae. Peristomium duplex: exterius dentibus sedecim, latiusculis; interius reticulatum.

MAsc. gemmacci axillares.

MxpNum. cAps. oblonga. Poristomium duplex: excerius dentibus sedecim, latiusculis; interius membranaceum, aequale, 
quale, laciniatum: laciniis latiuscue

lis, cum interjectis capillaribus.

masc. gemmacei in diversis plantis.

LEskia. caps, oblomga. Peristomium duplex: exterius dentibus selecim, acutis; interius membranaceum, par.. titum in lacinias aequales.

MAsC. gemmacti in diversis individuis.

NECKERA. CAPs. oblonga. Peristomium, duplex: exterius dentibus sedecin, acutis; interius ciliis sedecin distin. ctis, cum dentibus exterioris alter. nautibus.

MAsc. gemmacei in distinctis plantis. BUxBAUMa, cax's. ovati, obliqua, basi hinc ventricosa, depressa, in altera specie marginata. P'eristomium $\mathrm{d} u$ plex: oxterius dentibus sedecim, trun. catis; interius membranaceum, plicatumn.

Masc. discoidei.

poIYтисним. cars, oblonga, interdum quadrangula, apophysi quadran. gulae 
gulae insidens. Peristomium duplex: exterius dentibus 32 , brevihus, basi conlitis, incurvis; interius nembraua pluna, transversa, apicibus den. tium exterioris adglutinata. Calyptra pilosa.

MAsc. discoidei.

* perrozandoum. Hedw. stirp. 40. 1. p. 16. Iypni sp. Linn. Peristomium simplex sedecimdentatum. Flos ma-

- sculus femineusque alaris.

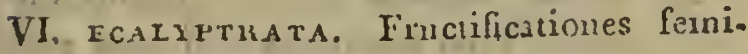
neae involutae calyptra; quae apice finditur capsulumque yel seminiforna corpuscula transmittit. Capsula apice, longitudinaliter, irregulariter de= hiscens foeta seminibus, funiculo in helicem torto s. e binis helicibus constructo elastico, plerumque adfixis.

28. Jungermannia. Capsula subglobosa unilocularis 4-valvis: apice deliscens longitudinaliter. 
CRYPT. ECALYRTKATA.

29. Marchantia. Capsula subglobosa unilocularis apice deliscens: dentibus suboctonis ; receptaculo communi pedunculato affixa.

5o. Targionia. Capsula subsessilis glo. bosa intra calycem hivalvem, sub apice frondis situm, pericarpium mentientem.

32. Anthoceros. Capsula longissima su. bulata apice dehiscens semibivalvis, basi calyce vaginata.

32. Blasia. Capsula obliqua frondi immersa, tubo extrorsum coronata per". sistente.

55. Riccia. Capsula subglobosa frondi immersa tubo coronata trajecto. Haec secundum octavam editionem Gen. pl. Lin. adnumerato Sphaerocarpo, audiunt: HEPATICAE.

VII. scetercata. Fructificationes femineac in scutellis orbicularibus, planis, convexis, radiatis rel nndis, marginatis s. immarginatis, vel spiraliter contortis, 


\section{Classis XXIV.}

$\begin{array}{lllllllllll}C & \mathbf{R} & \mathbf{Y} & \mathbf{P} & \mathbf{T} & \mathrm{O} & \mathrm{G} & \boldsymbol{A} & \mathbf{M} & \mathbf{I} & \mathbf{A}\end{array}$ r A D I C I I A (Miscellaneae).

Marsitea (Marsilie) Lin. Oen. pl.ed.8. 1614 .

Fructificationes radicales 2-3, pedicella. tae, subcompressae ovales.

M. quadrifolia, foliis quatemis integer: rimis L. Iuss. A. O. 1740. t. 15.

In aquis stagnantibus paludosis (Alsat. Palat. Salisb. Finnck; March. Brand. Loachimi; Bavar. Schranck, Schmidt; Francon. Frisclmann. -) Iul. Aug.

2. salvinia (Salvinie). G. pl. 1617 .

Fructificationes $4 \cdot 9$, glomeratae, inter. radices verticillatas.

s. natans, foliis oppositis simplicibus $\mathrm{L}$. (Marsil. natans) Mich. Gen. 6.58. Neck. A. P.3. 6.21.

III aquis stagnantibus, fossis (Palat. Bavar. Schranck; Siles. Matt. Barbi scholl. Berol. Wild. -) Inn. Aug.

5. prlularia (Pillenkraut) G. pl. 1619.

Fructificationes solitariae, instar pilularum inter folia et radicem subsessiles. 
* g. globulifera L. Bull. herb. 1.375. Bolt. fil. 40 .

Iu inuudatis (FInlsat. Megap. Brem. Oldenb. Hannover. Hanov. Erlaug. cic.) Iuu. Sept.

4. Isoxtes (Brachsenkraut) G. pl. 1620.

Fructificatio intra basin dilatatam foliorum.

I. lacustris, foliis subulatis semiteretibus L. Bolt. fil. 41. Fl. dant. t. 191.

In pratis paludosis (Holsat. Wigg.) Inl. Scpt:

\section{TEI, T A T A (Miscellaneac).}

5. EQUISETUM (Kandelwisch) G. pl. 1614 .

Spica ovata. Fructificationes peltatae, angulatae, inferne deliscentes, 4-7 loculares.

* 1. E. hiemale, caule nudo scabro, basi subramoso L. Bolt. fil. 5ag.

In sylvaticis nliginosis, Maj. Ang. (Vaginat breves apice crenulatae nigrae.)

*2. E. limosum, canle subundo laevi L. Bolt. Fil. 38.

In palndosis stagnantibus; Maj. Inu.

*5. x. Auviatile, caule striato, frondibus subsimplicibus L. Bolt. fil. 36 .

In fossis, ad ripas; Maj. Inm. (Utramqque specien conjungit Eih/hort et vocat: Equisetum Heleochariu, canliums aequalibus, snbramasis tcretibus, striatis, fistulosis viridibus; ramis 
sulssimplicibus 5 - sulcatis sterilibus; dentibus vuminarum plurimis subulatis acutissimis: vagimularum quinis, dorso minime suleatis Beitr. 2. 15g. Var. frondibus longissimis intemodia-ter quaterve superantibus, et frondibus subsedecim internodia non aecuantibus: Eq. polymorplume schranck, Donciirnoor, 91. 4. 1794.)

* E. pralustre, caule anculato, frondibus simplicibus I. Boit. fil. 30 .

In fossis, pratis paludosis; Maj. Iul. (Equi. selum polystrileyum, foliis apice spicatis, spica terminali majore. Ifri. Amgl. Ed. 3. ८. U. t.3. Equis. uodosum, stipite sulcato, ramo:o aphyllo; geniculis incrassatis, cum ramis norifero Schranck, Donaunnoor 9l.)

* 5. ₹. arvense, șcapo fruclificante nurdo, sterili frondoso I. Fl. lind. Bolt. fil. 34.

In agris, arvis; Mart. Apr. (Equiselum pros. tense, calulibus ramosis, sulcatis scaberrionis. Ehr7. Beit\%. 3. 7.7.)

*6. E. sylvaticum, canle spicato, frondibus compositis L. Bolt. ful. 32. 33.

In nemorosis; Apr. Maj. (Equis. capillare, frondibus tenuissimis compositis sterilibus.)

7. E. Telmateja, scapo fructificante den. se vaginato, fronde sterili ramoso-verticil. lata. Elrh. pl. crypt. exsicc. 31.

In nemorosis (Hanover., in monte Deister F $F_{2} r F_{2}$. Erlang. Schreb. Com.lipp. Schrader; Suttgart. Ful: pius Lihth. Jicitr. 6. 69. Hanoy. Gace rreer liken. Beitr. 5. 167. -) Miaj. Aug. (Specio iscina stirps cauli!us inacqualibus, teretibns leviluns glabris: fructicante simplicissimo, albo rubello mat- 
cescente; sterili ramoso, fistuloso - poroso, albo, ramis subquadragenis octosulcatis : sulcis alıerris majoribus; dentilus vaginarum subula. to-selaccis, scariosis: vagiunlarum quateruis, dorio sulcatis Ehr/2. Beitr. 2. $15 \mathrm{~g}$.)

\section{A N N U L A T A \\ (Filices.)}

6. POLYPODIUM (Tïpfelfarru) $G . p l$.

Capsulae distrioutae in puncta subrotun: da, per pagiuam frondis aversam?.

Fronde pinnatifida.

* I. P. vulgare, frondibus pinnatifidis : pin: nis oblongis subserratis nbtusis; radice squamata L. $F l$ lond. Bull. herb. t. 19y. Bolt. fil. 18.

In montosis nemorosis; Maj. Oct. (Var. pin. sis lobatis, proliferis Bolt. fil. 2. A. 5. a. b. Pol. cambricum. - Involucrum obsoletum, quod etiam in aliis Polyp. speciebus observatur.)

\section{Fronde pinnata.}

2. P. Lonchitis, frondibus pinnatis: pinnis lunulatis ciliato - serratis declinatis ; stipit. strigosis I. Fl. dan. 497. Bolt. fil. 19 .

In sy-lvis montosis subalpinis (siles. Carn. Alistr. Bavar. Salisb. Tyrol. etc.) Maj. Oct. (Livolucrum subumbilicatum.) 


\section{Fronle bipinnatifuda.}

*. P. Thelyrteris, fronde pinnata: pinnis pimnatifidis: pinnulis triangularibus re. Hexis. I. Schmid. ic. t. 11, i3.

In nemorosis lummidis; Inn. Oct. (Involncrum subrenitorme margine laciniatum.)

* 4. P. Phegopteris, fronde piunata: pinnis pinnatifidis, basi angustatis coadunatis. I. Bolt. fil. 20.

In sylvaticis, montosis humidis; ITaj. Oct. 5.'P. Marantae, frondibus hipinnatifidis: pinnis opposito-coadunatis subtus hirsntis. simis, basi sulsdentatis. L. (Acrost. Marantae.)

In rupibus abruptis (Bohem. In arce Tollenstcin Lusatiae contermina; iu moute Kleis, in ditione Furstenber, in Sigria Hatnke.)

* 6. P. Oreopteris, frónde pinmata: pinnis lanceolatis pinnatifidis: pinnulis integris oblongis obtusis. Ehrh. Bolt. fil. 22. (Pol. Thelypteris.)

In nemorosis montosis (Salisb. Funck; Berol. Willl. Hass. Vogler; Conn. lipp. Schrader; Saxon. infer. Hannov.Schwalenb. in monte Deister; $E / 7_{2} T_{2}$. Puatisb. Mair erc. Pol. moruantum Vogl. W'ill. Rrih.) InII. Inl. (Radices fibrosae copiosissimae, implicatac. Rhizoma basibus stipitum totum ob. tectum. Stipites sulbpaleacei frondes subbipinnatae. Pinnae primariae cusiformes, subpaten. tissimae, minime rellexae. Pintsae secundariae obtusae, integer rimae glomeruli seminum solitarii, snbinarginales, remotinsculi $E h r /$. Beitr. 4. 44.)

$$
\text { A } 3 \text { \%. }
$$


7. P. Callipteris; fronde pinnata: pinnis ovato - oblongis profunde pinnatifidis rel pinnatis: ponnulis uratis, ovato - oblongis, duplicato serratis: serraturis mucromatis. Elhr. pl. crypt. 55. Seguier. Veron.5. t. 1. fig. 1.

In nemorosis montosis (Mlegap. Timm; Han. nov. Ehrh.-) Inl. Ang. (Var. major serraturis 2cutis.

* 8. F. Filix mas, fronde pinnata : pimis pinnatifidis vel pinnatis: pinmulis oblongis obtusis cremulatis: stipite palenceo. L. Bolt. fil. 24. Bull. herb. t. 183.

In nemorosis; Maj. Oct. (Involucrum reni. forme.)

\section{Fronde hipimata.}

9. P. rigilum, fronle bipinnata: pinnulis ovato - Ianceolatis incisis: laciniis conniventi $2-5$ dentatis; stipite paleaceo.

In moutosis subalpinis (Salisb. Funck. stipite palescio, statura pyramidsa, pedili et ultra, colore ex llavescente viridi, acervulis frucific. crassis, rigidine et augustiore fronde, pimts pimulisque. differt a Polyp. Callipt. el dentato. nec non a Pol. rigido Syst. nat. ed.r3. (Acrost. longifol. 129i.) Involucrum reniforme dilute ru. fescens.)

10. 1. Filix femina, fronde bipinnata : pinnulis ovatis sermtis ohtusinsculis : serraturis 2 - 5 fidis. I. Bolt. fil. 25. Pluk. phyt. 181. fig.2. Bot. M Lag.9. fig.12.

III llcmorosis; MIaj. Oct. 
* 11. P. dentatum, fronde bipinnata: pinnulis ovato - lanceolatis incisis dentaris ; fructificationibus biseriatis crassis. Mull. Fridr: t. 2. fig. 5.

In sȳlvaticis montosis; Maj. Oct. (Affine P. trilisto excepta rigidiore textura, saturatiore co. lore, pinnulis et fructificat. majorilus. Involn. crum semiumbilicatum costam versus dehiscelss.?

12. 1. incisum, fronde bipinnata: pinnulis angustis, lineari-vel ovato-lanccolatis, margine argute inciso. dentatis, fructificat. hiseriatis crassis, demum confuentibus. Plak. phyt. t. go. Jig.4. Bot. Mag. l. c. fig. 15 .

Iu sylvaticis, umbrosis; Maj. Oct. (Involu. crum seminmbilicatum costam versus dehiscens.)

* 13. P. trifldum, fronde bipinnata: pin. mulis lineari. lanceolatis incisis: laciniis apice comiventi - trifidis; fructificat. spax: sis.

In nemorosis hinc inde cum sequenti, molli. tie ac habitu conveniens; Maj. Oct.

14. P. molle, fronde bipinnata: pinmulis Ianccolatis pinnatifidis: laciniis linearibus apice comiventi - serratis. Sehreb. Ehrh. pl. crypt. .

In nemoribns ad arborum radices; Maj. Oct; * 15. P. dilatatum, fronde bipinnata, sti: pite foliolis et piunis basi milatatis, pin* natifidis: pinmulis scrrato - incisis mucroA. if

patis 
natis linearibus; fristificat. sparsis in an. gulormm incisuris. Mull. Fridr. t.2. Jig. 4.

In sylvaticis montosis; Maj. Oct. (Stipite ad foliolorum conjughtionem el pinmis inferioribus dilatuls, pinuulisquc angustioribus facile a $P$. cristato distinguitur. Pol. aristatum Bellardi Append. ad flor. pedemont. $\because$. Mcni. de l'Acad. R. d. Sc. de Tuin v. 5. p. 155.)

16. 3. tanacetifolium, fronde bipinnata, stipite et pimis basi aequalibus, pinnatifidis: pinnulis linearibus inciso - serratis mucrouatis; fructificat. sparsis in angulo. rum serraturis.

In sylvaticis montosis hinc inde; Maj. Oct. (Iuvolucrum subreniforne.)

* 17. r. cristatum, fronde bipinnata: pinnis pinnatifidis, pimulis orato - oblongis duplicato - serratis : serraturis spiunso - ciliatis conniventibus. I. Flor. dan. 707. Bolt. fil. 23. MTull. Fridr. 1.2. fig.2. Bot. Mag. l. c. Jig. 11 .

In 11 emorosis montosis; Maj. Oct.

* 8. P. aculeatum, fronde bipinnata: pinnulis lumulatis ciliato - dentatis; stipite strignoso. L. Bolt. fil. 26. Nluk. plyt. 180. $\int_{0} \cdot 2$.

In sylvis montosis (Bav. Carn. Hercyn. Francon. Tyrol. Nascov. Yalat. ete.) Imu, Oct. (Pol. apprendiculatmm, fronde bipinnata, pinnulis lnumlais appendiculatis ciliato - deutatis. I'luk, jll $)^{\prime} \cdot$. 180. JLg. 1.) 


\section{(FItrcrs.)}

19̄. P. fragile, fronde bipinnata: pinnulis ovatis incisis crenulatis. L. Bolt. fal. 27 . Flor. dan. 401. Bot. Mag. l. c. Jig. 14. a, b.

In muris, fissuris rupinm; Iun. Sept. (Pol. denticulatum, frond. bipinnatis, pinnis ovatis obtusis incisis dentatis Dicks. Fasc. tert. $\%$. crjpt. t. 7. Jig. 1.

* 20. Y. anthriscifolium, fronde bipinmata: pinnulis pimatificlis, inciso-denticnlatis. Bot. Mag. l. c. Jig. 14. c. Pluk. phyt. 180. fig. 5.

In rupestribus, sylraticis; Inn. Sept. (Pol. angustatum, frondibus bipiunatis : pinnulis lanceolatis pinnatifidis, inciso - denticulatis ; fructificat. crassioribus. Bot. Mag. l. c. e.)

*21. 1. cynapifolium, fronde bipinnata: pinnis pinunlisque cuneiformibus, incisis denticulatis, venosis.

Hinc inde cum priori; Inn. Sept.

22. P. tenue, fronde bipinnatn: pinnulis pinnatifidis angustis margine denticularis. Bot. Mag. l. c. Jig. 14. d. Pluk. pliyt. 79. fig. 3.

In montosis; Inl. Sept.

25. P. fumarioides, fronde bipinnata : pinnis pinnulisque distantibus margine exscisis. Seguier. veron. 5. t. 2. fig.5. (quoad Inabitum.)

In montosis subalpinis; Inl. Sept. (Involu. crum in spec. 19. 25. ntplurimum operculatum s. conpexum sine umbilico.) 
24. P. alpinum, frondibus alterne tripiumatis, pinmlis oblongis, vage incisis: lacin. lis obtusis apice brevissime bifidis $h$ ulf. Iacq. coll. 2. 171. ic. pl. Vol.2. fasc.6.

In summitate alpium (lithopolitanarum $W^{\prime} u l f f$.)

25. P. pelicularifolium, froude bipinmata: pinmulis linearibus cremulatis: crenis cristatis; involucris semilunatis. Pluk. phyt. 180. fig.6. Bolt. fil. 2. fig. G. (Polypod. ilnacticum.)

In subalpinis, montosis; Inl. Ser.t. (Exccptö rolyp. fondiro (iil IIclvetia nimime raro, in Germinia vix unquau reperto) Boll. fil. 2. Plake: phyt. 89. Jig. 2. rhacticum ct regium Lin. incer. tae specics.)

\section{Fronde supradecomposita.}

26. P. montanmm, fromde tenela triangl-. lari miplicato - pimata: pinmulis pinnatif-. dis: laciuiis (subfalcatis) ofutusis argnte in. cisis. Allion. Pluk. pliyt. 8n. fig. 4 .

In sylvaticis subalpinis (Austr. Tyrol. Macn. ke. - Ad Cyatheam refertur a cel. I. E. Sruith in methodica sua disposilionc Filicum ab invo: lncro desmuta $r$. Mem. de l'scad. H. d. Sc. de Turin, v. 5. 1. 40. 4. 1795.)

* 27. Pr. Dropteris, frond. supradecompo. sitis, foliolis termis bipinatis L. Bolt. fil.
20 . Sept.

Ad arborum truncos, in fissuris rupinm; Iu1. 
7. ANOCIRA (Rollfarrii) G. pl. 1622. A

Capsulae sub frondis pinnis pinnulisque recurrato contractis.

* 3. o. Struthiopteris, frondibus sterilibus bipinnatifidis, fructificante bipinnatifida disticlaa. L. Flor. dan. t.16g. (Osm, Struthiop.)

In nemorosis montosis (Siles. Thuring. Her. cyu. Saxon. Tyrol. etc.) Iun. Iul.

2. o. Spicant, frondibus sterilibus pinnatifidis, tructifionte pinnata disticha. $L$. Flor. Lond. Bolt. fil. t. 6. (Osns. Spi-. eant.)

Li nemorosis mupestribus (Siles. Bavar. Salisb. Carn. Holsat. Saxon. Franc. Hass. Brem. Oldenb. Hannov. etc.) lVaj. Inn. (Blechnnm Spicant Smith.1.c.) Roth in Anealen d. Bas. 10. p. 36.

3. o. crifpa, frondibus sterilibus et fructificantibus supradecompositis; foliolis alteruis subrotundis incisis. I. Bolt. fil. t.7. Flor. dan. t. 490. (Osin. crispa.)

In sylvis humidis (Siles. JMacluschk. Hrzen. ke.) Maj. Iun. (Omnes species, nec non Orweclea sensibilis, quas examintavi capsulis gaudeus annulatis.)

\section{Pтzris (Saumfarm) G. pl. 1626 .}

Capsulae in lineam continuam, subtus cingentem marginem frondis, digestue. 
* x. aquilina, frondibus supradecompositis: foliolis pinnatis: pinnis lanceolatis, infmis pinnatifidis, superioribus minoribus. L. Bolt. fil. t. 10. Bull. herb. t. 207 . In sytvis, uemorosis ad margines; Inl. Aug.

9. Asprxnivм (Strichfarrn) G. pl. 1631. Capsulae in lineas rectas disco frondis subjectas, dispositae.

1. A. Adiantum nigrum, frondibus sub. tripinnatis: foliolis alternis: pinuis lanceolatis inciso serratis. L. Bot. Mag.9. 6.1. f. 7 .

In rupium fissuris; $A$ pr. - Oct.

(Adiantum lanceolatum, frondibus du. plicato - pinnatis lanceolatis : foliolis alternis, pinuis obnvatis-inciso-serratis. Huds. Mith. Bolt. fil. t.17. A priori piunulis latioribus obovatis, statura diversum.)

* 2. A. Ruta muraria, frondibus alterna. tin decompositis: foliolis cuneiformibus crenulatis. L. Bull. herb. t. 195 . Bolt. fil. t. 16.

Ad muros, in rupium fissuris; Iun. Oct. * 3. A. septentrionale, frondibus bifurcatis laciniatis linearibus. L. Bolt. fil. t.8. (A. crost. septentr.)

In rupium fissuris (Austr. Siles. Carn. Carinth. Bavar. Palat. Saxun. 'Illur. etc.) Inu. Oce. (Caps. aces: 
aeerruli in juniori stirpe distincta lineares, do. musn confincutes.)

*4. A. germanicum, frondibus pinnatis: pinnis alrenis cuneiformibus superne incisis, 2-5-fidis, Weis. Iacq. misc.2. t. 5. (Asplen. alternifolium.)

In muris, ad nupes ( $A$ ustr. Carinth. Holsat. Xassov. Lips. ctc.) Iut. Oct.

* 5. A. Trichomanes, frondibus pinnatis: pinnis subrotundis crematis. L. Bolt. fil. t. 35. Bull. herb. t. 185.

- Ad muros, rupes; Apr. - Oct. (Var. pinnis lobatis crenatis Bolt. l. c. c. 2. f. 2.)

6. A. viride, frondibus pinnatis: pinnis subrotundis crenatis, basi truncatis Huds. ivith. Bolt. fil. t. 14.

Hinc inde cum priori (in praefect. Lanenst. Ehrh. piant. cropt. 7s.) Inl. Sept. (Fronde ra." mosa Bolt. 7. c. t.2. f. 3 . Stipes viridis, basi fișcus.)

*. A. Ceterach, frondibus pinnatifidis: lo. bis alternis confuentibus obtusis. L. Bull. herb. t. 583 . Bolc, Jil. t. 12.

In fissuris rupium (IIercyn. Nassov. Carn. Tyrol. Francon. Norinb. Fanzer; prope Mil. tenberg ad Moen.; in moutibus Schaucnb. Whrh. pl. cropt.211.) lum. Ang. (frondes squamulis liu. scis reticulatis subturs tcctae.)

8. A. Scolopendrium, frondibus simplivi. bus cordato - ligulatis integerimais, stipi. B 2. 
tibus hirsutis L. Curt. R. lond. Lolt. Jul. c. 11.

Iu moutpsis asperis (Siles. Caru. Bavar. Szlisb. Palat. Voigtl. Tub. Thuring. Saalf. ad muros bei Grimme Baumg. prope Springen Fhrh. Thatisb. Hop) -) Ang. Seps. (Var. froudibus divisis, crispis. Involucra in spec. 1.6. costam versus, in Asplen. Scolnpcudrio sumura longiu. dinali dehiscuns, inde novinm Genus Scolope'1s. drium constimir cel. simsih. v. Mémoires de l'Acad. Y. des sc. de Turin 1. c. p. 410.)

\section{B I VA LVIA (Filices.)}

10. ophogrossum (Nattcrzunge) G. pl. 1621.

Spica articnlata disticlen: articuli trans: versim delriscentes, bivalves.

* ovulgatum, fronde ovata; scapo simplici. L. Bolt. fil. t. 3 .

In pratis, pascuis humidis; Mrj. Imll. (Var. spica composita.)

11. osmunda (Traubenfarrn) G pl. 1622.

Spica racemosa; capsulae subglobosae, transversim dehisceutes, bivalves.

*. o. Lumuria, fronde pinnata, scapo salitario, racemo laterali. I. Bolt. fil. t. 4.

Iu pratis, pascuis apricis: Maj. Iun. (Osmullda rarnosa, racemis latoralibus, frondibus lis. pimatis: pinuulis incisis sypt. nas. 1293. Irlor. dan. $t .29$. 
2. o. regalis, fronde bipinnata, racemo terminali supradecomposito L.' Bolt. fil. t. 5 .

In nemorosis bumidis (Siles. Holsat. Saxon. Francon. Brunsvig. Oldenb. Brem. Hanov. Berol. etc.) Ium. Ind.

\section{Lycorodiuxr (Bärlapp) G. pl. 2615.}

Fructificationes in axillis foliorum $v \in$ ! squamarum sessiles, bivalves.

*2. 1. clavatum, foliis sparsis filamentosis, spicis teretıhus pedunculatis geminis $L$. Dill. Hise. Muse. t.58. f. 1.

In ericetis montosis sylvaticis; IuI. Ang.

2. z. annotinum, foliis sparsis quinquefariis subserratis, surculis annotinis articulatis, spicis terminalibus glabris erectis $t$, Dill. $6.65 . f \cdot g$.

In sylvis montosis; Iun. Sept.

3. x. complanatum, foliis bifariis conna. tis: superficialibus solitariis, spicis gemir nis peduriculatis L. Dill. t.5g. f.5.

In sylvis montosis; Iul. Aug.

*4. r. alpinum, foliis quadrifariam im. bricatis acutis, canlibus erectis bifidis, spicis sessilibus tereribus L. Dill. t. 58. $f .2$. Flor. lapp. ed.2. t.11. f.6. Flor. dan. i.79.

In montosis subalpinis (Hercyn. Salisb. Tyrol. Carn. etc.) Iul. - Oct.

B 3

*5. I. 
5. I. inundatum, Inliis sparsis integrrvimis; spicis terminalibus foliosis L. Dill. t. fi. f. 7 .

In pascuis turfosis inmolatis; Inn. Scpe.

6. x. Selaco, foliis sparsis octofariis, caule dichotom, erecto fastigiato, caps!rlis spaz. sis 1. Dill. t.56. f. 1. Flor. dan. t. 104.

In sylvis montosis, cricctis humidis (Hercyn. Thuring. Megap. Duc. Oldenb. Palat. Kavar. Tyrol. Carn. etc.) Ang. Sept.

7. r. Selaginoides, foliis sparsis ciliztis Innccolatis; spicis solitaris terminalibus froliosis I. Dill. t.68. Flor. dan. 4.70. Hall. IIist. t. $46 . f .1$.

In cricctis, pascnis muscosis (prope Barbi, Vegesark Duc. Brem. Salisb. 'Tyrol. Bavar. Silcs. rec.) Inl. Scpt. (Capsularmu in hac specie duplex frums: inferiores apurudici lumblate insideut, Hede dolisccutes 4 ralves apparcut; corpuscula contiucut mucamenucea a foveola excavata; supesiores cxacte bivalves. Corpuscula intus fo. vent ut in relipuis tyc. ad lentem vero romu. dia, echinata, pollini antlerarum similia.)

3. r. raricans, foliis quadrifarie: linis mentis, binis imhricatis, spica filifninti hifurca, caule ramisque repentibus dicho. tonis compressis. Syst. Nat. ed. 13.1291. IBill. †. 65. f. ...

Ad radices arborn:n (Bavar. Scliranck, Tyrol. Salibl. Tiunek: L. hulluesicum ctc. Capsulac, dutplicis generis, pulvis in bivalvibus crocens. Spe. 
cies affines $\mathrm{L}$. helveticum ct denticulatum, scxu distinctac?)

\section{Cа Lу т т ат A (Musci.)}

73. TRENTEPOIIAA (Trentepolulie) Rotho Bot. An. 10, 53.

Corpuscula sessilia intra axillas foliorim, ovata, perichaetio cincta, clausa decidua.

T. erecta. Flor dan. 215

Jocis arenoxis inundatis (Ducat. Oldeul. Tren.

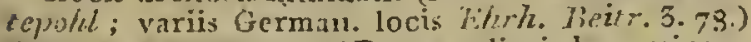
Acstate, Autumno. (De peculiari hac stirpe. de cujns uaturali Ordine, multo magis partibus fructificationis, usque ad hutc diem Botanici in. certi haerebant, observationes meas ad excmpla: ria ab ipso rev. I. F. Trelle ey/hl mecum quain humanissime communicata, institusas ac repctiiss; pancis hic enarrabo et annexa tabula illu. strabo. Suerculi vel caulc's adscendunt simplices, innovationilus tamen clongati, vel basi ramosi, plerumque masculi ; inferuc undiusculi saturate purpnirei, superne dilutioris coloris. Folia sparsa, in hac uvato - lanccolata, saturatius viridia (a a llativa et ancta masnimdin'), in illa stipe potins lauccolata, pallidiora (b b) i semiamplexicaulia, smperue confertiora imbricata, tameu erceta, carinata, dorso ex deusiori fasciculo du. culorum nervosa, apice ad leutem ecrulata et veuis anastomosantibus reticulat a, quarum lougitudinales recta excurreutes, aliis trausversalibus minnribus counectumtur. I\%,r mascielus (c c) in distincto individun, vel $\mathrm{c} x$ ba-i surculorum qui propazationis urgaua gerunt, cripituliformis, ex foliis compoutur perignntialibus, extimis cordato. lanccolatis, apice productis re. curvis, remote sermulatis, juterioribus ovalis 184

bre: 
brevioribus, basi purpurascentibus, rcticuli arec olis laxis rhomboidcis, apice vircscentibns, areolis magis coarciatis (d). Inter lacc folliculi spermatici (antherac fiediu.) ovato-cylindracei ? (c) paltidi coloris, mniloculares, apice dehiscen.. tes (f. folliculis evacuatur fuscescens vertosus), ejaculantes pollen granulosun; fere scusiles, per. quam mumeron: 1n.12; filis sncculentis (para.. jhysibus) clavatis articulatis: articulis superne: hrevioribus, pallide roseis, lntescentibus, etiam albidis muniti (s). I los fenineus (h) ctiam in sunumitate distincti individni, intermixtus pric ri, plerumque sterilis, capimliformis, ex longissimis folizs lanceolatis carinatis perigonialibus, scusim iuteriora versus decrescencibus, asctius quam in mascrlis reticulatis, structus, ad basin tantum tlalamo purpurascenti concoloribus. Ge. nivalia hapc amplexantur $6-8$, iustricta sty lis $c x$ violaceo dilute purpureis (i), duobus ductulis samratioris cololis, opere celluloso transversali conjunctis, demum confluentibns, percursis; tigma lubac in modum ampliatum, nou raro Inciriatum. Cum stylo immediate calypera cont. licxa, ovariun, germen f. capiulac undimeutum cingit oblonsum, etiam, sed dilutus, coloranim, brevisaime pedunculatum, reti vasculoso ejus. dem formac ac in stylo, magis tamen ab increincuto ovarii distenso, scptum. Rlia succentu. xiata (sterilia organa femiuea $H_{\text {edw. }}$ ) illis immiccentur, pallide viridnla, acquali fere latitudinc, infernc parmu latiora, supernc obtusa, cellulo. so. gramulosa $(k)$. Fila succulenta ciiam adstant non panca, lineari. lanceolata, articulata, de. colora ( 1 , mum $c x$ his resiculis longitndinalibus intcrceptum). Gorpuscrela illr, pro capswlis venditata, exigua, tamenz mudo oculo couspicus, intra axillas foliormen sessilin vel potins laereu? lia $(m)$, ovala, in prima actate cx viridi. dein ex rubro, demum ex tusco purpurco colorata, intumescunt, nitent, foliolis uudique inclusa 
- Juli.t.
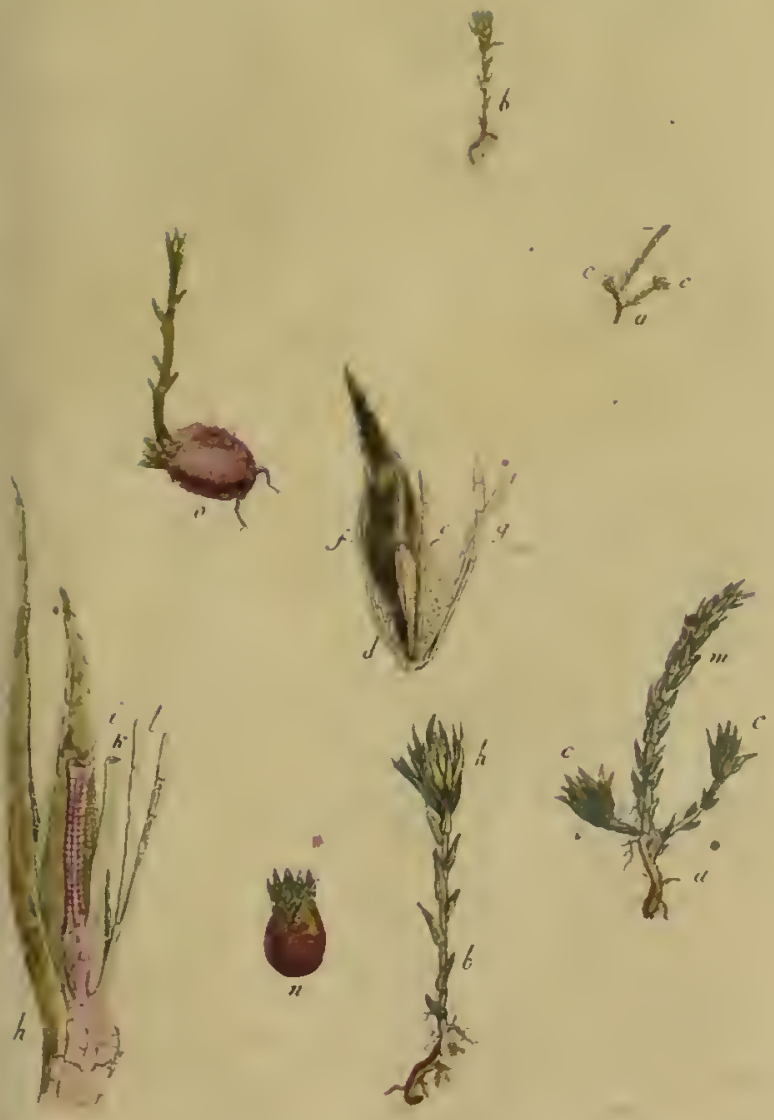

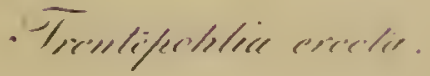



imbricatis, ea qua parte ultra corpusculum pro. minent viridibris, pellucidis, reticnlatis connirentibus vel patentibus (11), inferne corpusculo adnatis, concoloribus, reticnli areolis subrotumdis. Tacur facillime matura desiliume. Semina intus obserware nou ponni; at inter surculorum ronfertissimas et capillares radiculas quaedam accurrebant, quise ex apice folionum novum sur. culun emittebant, foliolis et radiculis instrucumm (o). An igitur potius vivi partus? cum capsulae verae in apice distincti individni ses. siles sirut hucusque practervisae? Anne simile guid accidat, ltt in intis stirpilus, quanum vita partum semel perfecum excedit notellis surcu. lis, gemmis, bulbillis in spero sequentis anm propagandis? Vel Frucrus ideo raro mature. cant, quia,-more bulhosarum, omnis vegetatio. nis vis ul hulbos deferatur? -)

\section{1iuscin (Olitumand) G.pl.1636.}

Capsula sibessilis clausa cum rudimento operculi.

*. 1. crispidatim, caulescens, foliis ovatis cuspidatis patulis: termiralihus erectis conniventibus. Schreh. Phasc. t. 1: fig.1, 2.

In agris, hortis, ad fossas; Mart. Apr.

* P. piliferum, canlescens foliis oblongis piliferis erectis Schreb. Phasc. s.1. fig. 6,7

In muris argillaceis; Maj. Inn, *3. subulatum, caulescens, foliis lan. ceolato - Linearibus patulis Schreb. Phasc. p.8. Hedw. Musc. 1. e.35. Flor. dan.249. fig. 2. Flor. lond.

In arenosis ad aggeres, vins; Mart. Inn.

$$
\text { B } 5 \text { 4. }
$$


4. P. crispum, caniescens, foliis lanceoln: tis longe acuminatis revoluto - consortis. Hodu. Misc. 1. t. 3 .

Jocis pinguibus, ad semitas tenui gramine restilas gregation; fun.

P. paters, caulesceus, fọl. lanceolatis ser. ratis patnlis. Hedw. Musc. 2. t.10:

Locis udis umbrosis; Maj. Iuu.

6. P. mitidum, subcaulescens, foliis snbulato - calinatis subfasciatis. Hedu. 1. t. 54. Dicks. fasc. 1. c. 1. fig. 3. (Pliasc, axillare.)

In ericetie, solo glarcoso humido; Ium. InI (capsulis pedumculatis subemersis Dicks.)

*7. P. curvicollum, fol. subulato-lanccola. tis parmlis, capsulac pedunculo incuryo. Elirit. Hedio. 1. t. 11.

In depressis 6abnlosis; Maj.

+8. $P$. serratum, acanle foliis ovato - lan. ceolatis plauis serratis erectis Schreh. Phase. c. 2. fig. 2, 2.

In pratis pinguibus limosis, solitaric; Vere Aṇtumnono.

*?. P. volutinum, acaule, surculis filamentaceis geniculatis zamnsis. Coliis ovato-lancenlatis serratis. Dicks. fasc. x. t. 1. fig. 1. (Phasc. serratum.)

In pratis Rysso filmenfosac rel Coufervac similis; Vere, Aumunuo. (I'hasc. sto?nifervm, surculis filamentaceis gcuiculatis ramosis, fotiis peri. 


\section{(arsci):}

perichaetia lanceolatis dentatis Dicks. fasc. 3. t. S. jig. 2. halitu prioris, statura paulo majore, sur? culis interstitioscue longioribus fuscellis.)

10. P. muticun, acanle, foliis ovatis muticis concavis conniventibus Sohret. Whase. to 1. fig. $12,12$.

In agris, ad fossas, sparsion; Mart. Apr.

\section{BuXeauira (Buxbaimie) G. pl. 1659 .}

Capsula ovala olliqua basi ventricosa.

1: n. Soliosa, acrulis, capsula subsessili foliis cincta 1. Sclmial. Buxl, fig. I. Flor. dan. 249: Hedie. fund. t. 9. Jig.51.

In syivaticis al tor:am pinguem, arborum. perrarum radices; Aug. Sept.

* 2. B. aphylla, pedunculo elongato, capsula altero latere depressa margmata. I. Sclmid. Buxb. fis. 11, 12. Helle. fund. 6. 9. fig.52. t. 3 . fig. 10. Hor dan. t. 44. MTartinis allg. Gesch. der Nat. 10 Th.589.

In terra ex putrefuctis plaxiis orta dcclivi, locis sylvaticis; Ian. Apr.

16. SHHAONUM (Torfmons) O. pl. 165\%:

Capsula apopliysi orbiculari insidens. saepe calyptrac parte inferiore abrupta basi cincta.

* 1. s. oluturifolium, ramis deflexis, foliis ovato-cordatis ohense acntis, concavis. Dill. Miusc. t. 32. fig. 1. Gri. erypt. 241. 
In aquis, fossis: Iur. Ang. (s. renellum catr. le foliis et capsulis minoribus. Schmid. ic. $t .58$. fig. 6. (quoad capsulac liguram.) Ex Heroyn. Pers.

*2. s. intermedium, ramis deflexis, foliis ovatis oblongis concavis acutis. Dill. Musc. t.32. fig. 2. A.

In paindosis, uliginosis ericetis; aestate. (Vay. angustioribus ramis et capsulis, foliis acumina. tis, mayis adpressis, caule humiliore; color frequenser rubescit. Sphagn. acutifoliurn $E h r h$. crnpit. 72.)

5. s. cuspidatum, ramis deflexis, foliis". subulatis patentibus, planis undulato-acitminatis. Dill. Musi: t.52. Jig.2. B. Ehrh.: cr ype. 251 .

In aquosis fluitare solet, canlibus ad peda. $1 \mathrm{~cm}$ et ainplius longitudinem extensis. Acstate.

17. splackinum (Blasenmoos) $G$. pl:

Capsula apnphỵsi maximae subglobosac s. $11 \mathrm{mbraculiformi}$ insidens ; (peristomium simplex̃: dentibuis octo geminatis.)

*. s. spharicunt, foliis spatulato-acuminatis, apopluysi globosa. L. Hedw. Musc. 2. c. 16 .

In tirfosis (Hercyn. Ehrh. pl. crypt. 26i.) *2. s. vasculosum, apnpliysi suluglobösa banguinea. I. Hodu. Musc. 2. t. 15.

In paludosis cespitosis (Bruct. Hannov. E/hr/l.) 5. $s$. 
5. s. ampullaceum, apoplysi inzerse arm: pullaeforni colloque longo virente. I. Heduv. Missc. 2. t. 14.

In paludosis turfosis (Austr. Saron. Francon. ivegap. Oldeub. Fraucof. Lip\&. Salisb. etc.) Jul. Aug.

4. s. ligulatum, apophysi inverse conica attenuata.

- In rupestribus (Hercyn, Persoon) ineunte Au. tumno. (Hujus loci : Spl. turue Dicks. fasc. 2. rab. 4. fig. 2: folits ovato - oblongis (in nostra folia superiora potius ovato. spatulata, setae breviore (yiam in icone Ljicks.) - Spl. froeli. chianum Iledw. NIusc. 3. t.40: fol, inferioribus lingulatis, superioribus spatulatis obtusis (in uostra aciutis.) Peristominm octo paribus dentiiun ut in reliquis spec. siccitate reflexis, transver. sint tenuissinc striatis. Caps. oblongo pyrifor. mis, luci obversa apophysis inverse contica.)

18. Porrrmichus (Miderthon) G.pl. ${ }_{2} 660$.

Capsula interditm apoplyysi quadrangulac insiders. Calyptra ex apice deorsum pilosa: (peristomium simplex: dentibus plus quam 30 , membranula apice connexis.)

* 1. Y. commune, caule simplici, capsula parallelepipeda. I. Dill. Musc. t. 54. fig. 1.

In sylvaticis uliginosis; Inu. Inl. (Foliis om. wibus serratis. Pol. yuccaefolium Ehrh. frgn". 214. Perịtọmịun dẹntibụ! 64.) 
* 2. 13. juniperifoliu:n, canle et foliis bre. vioribus 1 igidis mucrnnatis. If illd. (P. juniperinum.) Dill. Mlusc. 1.54. fig. 2.

Locir siccioribus, pascuis nligino:is; Inn.

* 3. prififermm, canle simulici, folis integerrimis piliferis capsula parallelepipe. da. Schret. Dill. MTusc. 1.54. fig. 3. vere.

In cdisiorilus apricis, ericesis; Autumno,

* 4. P. alpirium, caula ramoso, foliis serratis, capsula ovata obliquata. L. Dill. MTusc. t. 55. fig. 4 .

fin montosis subalpinis (Hercyn. Weeb. Ehr/.)

5. 1. urnigurum, caule ramoso, foliis ri-j gidis subserratis, capsiala oblonga erecta. L. Dill. Mtusc. r. 55. fig.5.

Tu sylvaticis mnutosis (Hercyn. Nassov. MLe gap. -) Vere, Autumuo.

6. P. aloides, foliis lineari Ianceolatis plauis, apice serratis, capsula cylindrica obliquata. Hedw. Musc. 1. t. 14. Dill. Musc. t. 55 . fig. $_{\text {g. }}$.

In aroillosis nmbrosis; Antumuo et Tere. (Pol. manun. folitis lingularis plunis undique ser. rati-, capsua ovata erecla, operculi rostello breviore. Hinc inde cum priori.)

7. P. ericoides, foliis linearihns carinatis siccitate incurvis convexis oisscure serra. tis, capsida subrotunda demum nutante. Hedw. 


\section{(MUSCI.)}

Hediv. Musc. 1. t. 15. (P. nanum.) Curb. R. lond. (Pol. subrotundum.)

In ericetis; Ilieme, Vere.

29. ontwotuicuun (Goldhar) Hedw. IVIusc. II. 96. G. pl. 1648. (Meissia.)

Calyptra sursum pilosa.

* 1. o. hercynicum, foliis Ianceolato-linearibus concavis incurvis, capsula erecta urceolata. Hediv. Musc. 1. t. 15. (Polytr. liercynicum.)

In montosis (Hercyn. Ehrh. Catharinea her: cyuica. P1. crypt. 11. Bavar. Schrank.) Inl.'

* 2. o. crispun, foliis lanceolnto - Jinearibus confertis siccitate crispis, capsulis pedunculatis ex ampliata seia obovatis. Hedu: Musc. 2. t.35. Hill. Musc. t.55. Jig. 1 .

In saxis, arborum truncis; Tebr. Maj. (Pcri. stomium extrmun : dentibus 16 seflexilibus; ind ternum totidcrn processulis filiformibus erecti. libus.)

5. o. enomalum, canle rnmoso, foliis lanceolato - cariuatis patulis, capsulis pedunculatis oblongis stilatis. Hedw. Musc. 2. t.37. Dill. MIusc. t.55. fig.9.

Ad saxa, arborum truncos; Mart. $\Lambda$ pr. (Peristomium dentibus 16 unius ordinis.)

* 4. o. striatum, canle rarrosissimo, foliis lanceolato - carinatisi patulis, summis apice 
co crosis, capsulis oblongis subpedunoulatis. Hediv. MTusc. 2. t. 36 . Dill. Musc. t. 55. fig. 8.

In saxis, ad trumcos pueridos; Maj. Inn. (PC. ristomium intcrum ciliato - articulatum.)

*5. o.affine, caule subramoso, foliis lanceolatis patulis; capsnlis sulsessilibus oblougis sirintus. Sclirader Spicil. 67.

In ascribis $\mathrm{el}$ arborum truncis hinc inde cum antecedentibus : Hieme, Aestatc. (peristo" minm lnternuru capildaccum.)

6. o. cupulatum, caule superne ramoso, foliis lanceolatis patulis, capsulis subsessi. libus oblongis striatis, calyptra semiglobo: \$a. Dill. Mitusc. 6. 65. $\mathrm{jig}$. 10.

In arborum fruncis, saxis hinc inde; Autum. no. (Peristonitun: dentibus 16 unius ordinis, introllexis.)

* 7. 0. diaphanum, foliis lanceolato - acuminatis apico diaphanis canescentibus, caprula subsessili oblonga striata. Schrad. Spicil. 6g.

In asseribus, arbonum truncis; Vere. (Peristomii dentes iuterni capillacei cum exterioribus alternantes.)

20. ENCAzYPTA (Glockenhuth) G. pl. 1643.

Calyptra campanulata, magna, laxa, glabia, 
*. E. vulgaris, calyptra laxa conica aequali, varinulae inembranula pileiformi. Hedw. Musc. 1. t. 18. (Leersia vulgaris.) Dill. Musc. t. 45. fig. 8 .

Ad muros et terram; Apr. Maj.

*2. ‥ ciliata, calyptra laxa conica, ore laciuiata, vaginulae membranula cylindrica Hediv. MTusc. 1. t. 19. (Leersia ciliata.) Dill. IVluse t. 45. fig. 9 .

Ad saxa, lapides, aggeres; Iun. Iul.

2.1. Grinostomum (Kahlmund) G. pl. 1638.

Peristomium nullum.

* *. ovatum, foliis ovatis concavis pili. feris; capsulis ovatis. Hedw. Musc. 1. t. 6.

In muris, agris limosis: Apr. (Pottia cavifolia Ehrh. Beitr. 1. 187. Foliis cauescentibus statim a sequenú distingninur.)

* 2. G. truncatulum, foliis explanatis api. culatis, capsinlis truncatis. Hedw. Miusc. 1. t.5. Dill. Musc. t. 45. fig. 7. (Br. truncat. L.)

In campis, agris, ad fossas, seper; Hicme, Vere. (Pottia eustoma major, pyxidio ovali : Portia enustnma minor, pyxidio subgloboso Ehrh. Beitr. 1. 189.)

*3. o. pyriforme, foliis explanatis ovato. lanceolatis patentibns, capsula obovata. Dill. Musc. t. 44. fig.6. (Br. nyriforme 1..) 
In humidis, ad foseas; Febr. Apr. (Pottia pyriformis Eirth. 1. c.)

4. c. Heimii, caule erecto, foliis spatt1ato-acuminatis apice denticulatis, capsulis oblongis erectis, operculo oblique rostrato. Hedw. MTusc. 1. t. 3n.

Ad latera fossarum (Bcrol. prope Spaudau Heim; Megapol. Timm. -) Maj.

* 5. c. curvirostrum, caulilus elongatis palLidis subramosis confertis, foliis subulatis canaliculatis in siccitate recurvis. Hedw. Musc. 2. t.24.

In rupilus gypeaceis (Hercyn. prope Ostcrode et Niedersachswerfen Lirh. pl. cropu. 93 : Pottia curvirostra. Brymm fascicularum, capsulis erectis ovalis nndis, surculis ramosis, foltis capillaceis interrupte fasciculatis smbrecurvis Dicks. fasc. 3. 3.)

6. G. Osmundaceumi, canle simplici fron-. *oso pinmatifido, pinnato, folis distichis: Ianceolatis integerrimis. Dicks. fase. 1. c.1.. fig. 4. (Mn. Osmundaceum.), Hedw. Muse. 1. t. 29 . (G. pennatum.)

In cavitatibus aggerum, sub scpibns antiquis, solo pingui (Hercyn. Pyrmont. Misn. etc. Dick.. onia pusilla E/rrh. pl. crypt. 65.) IMaj. Iun.

* 7. G. Hedwigia, caule ramoso, foliis ora-. * - lanceolatis concavis, apice diaphano den-. iculato, perigonialium ciliato. Hedw. Musc.. 
1. t. 40. Dill. Musc. t.32. Fi..5. (Br. apocatpum $\beta$ L.)

In montauis supra saxa; Alutumno, Vere. (Hedwigia ciliata Ehrh. allodon Ej.Beitr. 3. 178.)

8. c. equaticum, caule tenui declinato, extremitatibus foliisque linearinus acuminatis reclinatis, capsulis oblongis. Hedw. Musc.3. 1. 11. (Hedw. aquatica.) Jacq. flor. austr. 5. t.290. (Hypr. aquat.)

Scopulis aquarum Auentium submersis innascitur (ad pedes alpium Schneeberg et Brey" Anstr. Jaç. Etiam ex Tyrol. subalpinis accepi. A Fontinali falcata distiuguitur deficiente peristomio. et fasciculo ductulorum satis crassum nervum in foliis angustis efficiente.)

*9. c. lapponicum. foliis elongato lanceo latis siccitatc crispis, capsulis striatis. Hedw. Musc. 3. t. 5 .

In nemorosis (Hercyn. primum legit Ehrh. Beitr. 7. 182. - Schrader.) Antumno.

10. c. microstomum, foliis e latiori basi lanceolatis siccitate crispulis, capsula oper. culoque obliquis, ora exigua. Hedw. Musc. 3. t. 50 .

In ambulacris sabulosis nmbrosis ; in piscina lutosa ex-iccata (Hannov. Ehrh.) Vere. Feminea genitalia masculis juncta, in eodem judivi. dvo spec. $7-9$; in distincto spec. $1-6$. 


\section{TET RA HIIS (Vierzabn) G. pl. 1659 .}

Peristomium simplex: dentibus quatuor pyraiudalibus; calypera plicato - striata: glabra.

*. pellucida. Schmid. ic. 3. (Muinm। pellucidim L.) Dill. Musc. t.51. fig. 2. Hedu, fund.2. t. 7. fig. 52.

Locis umbrosis humidis; Apr. Maj. (Georgia Mnemosynum E/urh. Beitr. 2. 188. flos masc. ca: pituli - vel disciformis in distincto individuo.)

23. Bमणㅡ (Stamnimoos) G.pl. 1640. 1647.

Peristomium simplex: dentibus plus quam 4; calyptra glabra capsulae insidens; setx terninalis; Hos gemmi- vel capituliformis. (Caulis erectus.)

\section{Dentibus 16 indivisis.}

I. B. apocarpum, capsulis subsessilibus terminalibus, calyptra minima. L. Hedwo. MIusc. 1. t.39. (Grimmia apocarpa.) Dill. Missc. t.32. Jig. 4 .

Super arborum truucos; Autumuo, Vere.

- (B. apocaulon, capsulis subsessilibus termiualibus: caule brevi subsimplici. Schmid. icon 4.57 . fir. 1. (B. apocarpon).

In muris, saxis; differt a priori operculi acumine longiore, caule et loco matali. Folia peri- 
gonialia apice caua; in specim. ex America se. ptentr. obtentis viridia.)

* 2. B. cribrosum, canle simpliciusculo, fol. lanccolatis confertim imbricatis patulis, summorum apicibus canis, peristomii dentibus pertusis. Hedw. Musc. 3. t. 31. (Grimmia cribrosa.)

In Hercyziac, Hassiae montosis (Persoon, Schra. der) Majo.

* 3. n. recurvatum, capsulis pyriformibus, pedunculis recurvatis, foliis setaceis. Dicks. 2. 7. Hedw. Musc. 1. t.38. (Grimmia recurvata.)

In nudis rupibus (Carinth. Klagenf. Wulfen v. Jacl. Mi.sc. 2. p. 96 . t. 12. Br. setaceum.) Vere, Autumno.

* 4. B. pulvinatum, capsulis subrotund is, pedunculis reflexis, folíis piliferis. L. Dill. Mase. t.5o. fig. 65.

In rupious ad muros, saxa, vulgare; Vere Autumn.

* 5. B. lanceolatum, capsulis crectis ovatis, caule erecto simplici divisoque, foliis lanceolatis concavis aristatis. Dicks. fasc, 3.4 . Hediv. Musc. 2. t.23. (Leersia lanceolata.)

Ad aggeres, supra lapides, in muris argillaceis Lips. Hedwix, Megap. Timm - - vulgare per ornem Germaniam; sed latuit procul dubio ideo. quod habitu, herba, capsulis operculo perquam similis sit haec opecies Gymostomo erruccato, C 3

c11\}ง 
cujus et consortio saepe fruitur. Hujus loci est etiam Halleri plantula Hist. helv. 11. 1809, \$1b Brvo viridulo Lin. citala, ut ex specimiliblis quise Hullerus ad IVillichium misit, video.) Als. tumuo, Vere.

6. B. Starkeanum, caule lineari, folis lanceolatis acutis apiculatis, capsula ovata recta, operculo obtuse conico. Hecluv. Musc. 5. 6. 34 (Neissia starkeana.)

Inter Gymnost. truncatum (circa Grols-Tschir. ma pouc Lissam polon. Sirarkic.) sutumuo.

7. 8. Dicksoni, capsulis erectis ovato-cylindraceis, caule 1 amoso, foliis linearibus carinatis siccitate subcrispis. Dicks. fasc. 3. 4. 8.7. fig. 7 .

In liguis, arbornm truncis emornis ; Hiene. (weissia Dicksoni Willdenon, prodr. go7.)

8. B. crispatum, capsulis erectis nvato. oblongis, ranle ramoso. foliis linearibus canaliculatis contortis. Dicks. fasc.3.3. t.7. fig. 4 .

In paludosis (Hamnov. Ehrh. pl. crypt. exs. 438. (A.́uclia cirrata.)

* 9. 3. curvirostrum, capsulis erectis ob. longis, operculi rostro subrecurvo, fuliis Jatcenlatis parnlis, siccitate crispis. Dicks. fasc. 2.7. Hoduig Musc. 1. 1..7. (TVcissia recurvirostra.)

Gregatim locis sylvaticis arenosis, lapidosis Chemnitz. Lips. Iledivig, Berol. Willd. Hegap. İmm $\rightarrow$ I I $)$ Aug. 
10. B. nigritum, capsulis sphaeroideis cexnuis minutulis nigris splendentibus, caule subdiviso erecto foliisque cordato acumiuatis nigrescentibus. Hedlw. Musc. 3.t.5g. (Veissia nigrita.)

Locis argillosis humentibus (circa Oberdorf Algoviae Froelich - B. rigratumL, capsulis cermuis ovals denticulatis, foliis lanceolatis acutis cariustis, surculis fasciculatis Dicks. Jasc. 3. 9.)

* 11. B. VI eissia, capsulis erectis oblonro ovatis anmulatis, foliis secundis linedri subu. latis rigidis. Dicks. 2. 5. Hedw. Musc. 1. 5. 8. (IVeissia heteromalla.)

In sylvaticis ad vias cavas (Chemnit. Hedw. Hercyu, ihrh, plant. cojpt. exsicl: 17j. Afzelia heteromalla.) Maj. InI. (Capsula erecta e? dentibus indivisis a Br. heteronallo, siniplicibus * B. capillaceo valde simili distiugltendum.)

* 12. 13. pusillum, capsulis ovatis crectis, canle simplici exigno, foliis capillaribus rectis. Hedw. MLusc. 2, t. 2y. (Treissia pu. silla.)

In duris lapidosis (Hamnov. Ehrh. pl. crypt. exsicc. 183. Afzelia pisilla.) Vero, Autumno.

13. B. rubellum, capsulis nvato-cylindraceis exectiusculis, folitis lanceolatis erectis patulis cum nervo rubellis, siccitate contot tis.

Inter Encalyptam vulgarem frequenter occur. rit; Vere, Autumuo. (Caulis simplicineculus rit; Vectus, superme toliis imhricztis tectus, brevis,
$\mathrm{C}_{4}$ 
seta terminalis vel lateralis semuncialis, purpurascens cum caule; capsula parva; peristomii destes 16 , e latiuscula basi angustati, margine
sublenticulati.)

* 14. B. concroversum, capsulis ovatis erectis, fol. acute lancenlatis siccitate contortis. Heciw. Musc. 5. t.5. (Weissia controversa.)

Locis sulmmontanis, ad scmitis declives grege

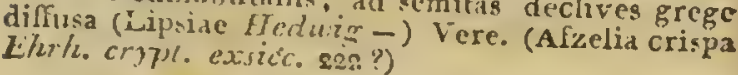

15. B. viridulum, capsulis erectis oratis foliis lineari. lancenlatis acmuinatis imbricato-patulis. I. Dicks. fasc. 1. 3. t. 1. f.5.

Locis Inmidis, umbrosis; Tere.

* 26. 2. Inteolum, capsulis erectis oblon. cis, foliis (in siccitate) linearibus crrioalis, crispis. (Meissia crispa Tim. prodr. 756$.

In igris, azgerilus gregatim; Vere. (Cansula ohlouga, oblongo. ovata, scta lougior lutescens miavis. quam iu $B$ controverso. Hujus Inci videwmr: Br. viridulum (et paludostun) quonmdam; Br. vireus IJicius. Br. viridulum Cur.

\section{Dentiluns 16 dirisis.}

17. B. parrulum, capsulis erectn-crmuis, canle erecto, foliis erectis capillariluns rigidiusculis enerriis. Dicks. fasc. 3. - I Icriw. ITurc.2. t. so (Dicranum prsillum.)

Berolini ("iildenau) I" uliginosis umbrosis (Hercyu. Schrader.) Autumno. 
18. в. simples, capsulis nvatis cernuis, foliis suhulatis, canle simplici innovatiourbus ranuso, medio pedunculifero. I. Hedw. Musc. 2. i.5\%. (Dicranm varium.) Dill. Misc. t.5r. fic. 5c.

In agris humidinsculis, argillosis; Autumno, vere.

19. ז. Ovale, capsulis oralib's operculoque recis, canle crecto subsinplici ramosoque: ramis fastigiatis, folris Inncenlatosubulatis, pilo cano termiuatis. Hedw. Musc. 5. t. 34. (Dicianum ovale.)

In montosis, densis saepe cespitulis (Saxon. Hedwig.) Vere.

20. 3. aquaticum, capsulis obliquis suba cerunis, caule erecto subsimplici ramnsoque: ramis fastigiatis, foliis acute lanceo. latis, siccitate contortis. Ehrh. crypt. exs. 213. (I)icranum aquaticum.)

\section{Hannoverae (Ezh/hart.)}

21. D. virens, capsulis incurvis obliguis, foliis e lasi subvagiuante llexunsa lanceo. latis acutis carinatis. Suartz. Hedw. Musc. 3. 32. (1)icranum vireus.)

In sylvis I apponiae Finlandiae collegit $O$. Siun $1=$; In paludo is Sylvaticis (Kissoviace ideers: Br. pelincidum, ex fide exemplarinm eodem indicato loco collectorum - ) Antumno. (Dillenii figmra 23 , tab. 6. fir. pellucilum L, hanc speciem nun in to cxprineret. Operculum conicum aguminatum obliqmum.)

$$
\text { C. } 5
$$


* 22. B. purpureur, capsulis obliquis cernuis siccitate striatis, operculo conico, foliis lanceolats carinatis. L. (Mn. purpureum.) Dill. MLusc. t. 49. fig. 51.

In eylvis, hortis, tectis, muris, ad vias; Vere (sequentes sub:pecies frequenter occurmunt: B. Longricaule, capsulis obliyuis, foliis lanceo. atis acuminatis, caulibus setac longitudinis. B. Zirenicrale, capsulis obliquis suberectis, foliis lanceolatis capillaccis, scta caule duplo triplove longiore, subdicholomo, vel simplici. Dill. Jiguraj. E. Deiltes per paria transversim consicxi.)

25. B. bipartitum, capsulis erectis subobliquis, surculis hifidis, foliis lauceslatis acutis carinatis imbricato-patulis. Dicks. fasc. 2. 7. Dill. Musc. t. 49. $\sqrt{i g} \cdot 50$.

\section{Ad aggeres; Vere.}

* 24. 23. Sirictum, capsulis obliquis cernuis. foliis alternis subnlatis strictis, canle ra: moso dichotomo. Dill. Musc. 1.49 . fig.52.

Locis humidis turfosis; Vere, Autumno. (B. purpureo longicanli, exceptis angustioribus fo. liis cauli nagiv undo et ramoso in siccitate valde adpressis simile.)

* 25. ח. struniferum, capsulis erectiusculi *ubpyriformibus basi intericre strumosis; foliis luncenlato - lincarbus subsecundis siccitate torilihus. Dich.s. fasc. 3. 8. Hodw. 31usc. 2. 6.52. (Tissidous strumifer.) 
In pratis uliginosis. Ad saxa et rupes (iu moun te Bructero Hercyu. Sichrader - Dicranum stru.

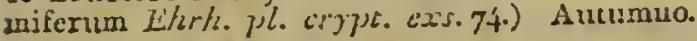

26. 3. polycarpum, capsulis ovatis ere: ctis subobliquis, operculo incurvo, folies linearibus sicciate inrillbus. Hedw. Musc. 2. 1.31. (Fissidens polycarpus.)

In sylvaticis (Hcrcyu. E/srfecert, $\gamma l$. cropt. exs. 8\%. Dicramum polycarpum.) - (quoad habia tum, excepta operculi direcrione respoudet: Jizg. 41. rab. 48. Dill., B. polmphillurs, capstllis erectis oblongo ovatis denticulatis, surculis ramosissimis, foliis confercis lauceolatis siccicate crispis. Licks. Jusc. 3. 7.)

*27. B. aciculare, capsulis esectis: opeqculo aciformi recto, folits confertis erecti subsecundis. 1.. Heilu. Musc. 2. t.35. (Dicranum aciculare.) Dill. Musc. t.46. Jis. 25.

In montosis ad saxit alvcorum fluviorumque; vere. (B. rivulare, follis patulis, canle diffuso, Dill. Miesc. tab. 46 . fig. $26 . \mathrm{Br}$. aciculare aquavicum IVeb. spicil. St.)

28. B. Pagellare, capsulis erectiusculis subcylindraceis : operculo longe rostrato incurvo, foliis subulatis canaliculatus secundis, caule ramoso interrupte flexunsn. Dicks. fasc. 3. 6. Hedw. Musc.5. t. 1. (Dicranum Hagellare.

In sylvis, in truncjs arborum dejectis (Primun detexil Tirnm.) In rupibus etiam, varis Germania locil collectam habeo; Autumulo. (Fi- 
gura Dill. 38. tab. 47. Bryum pilo:nm interrupte falcatum, habitum non male exprimit.)

*29. 3. heteromallum, capsulis ovatis obli-. quis, foliis setaceis secundis. L. Hedu'. Musc. 1. t.26. (Dicranum heteromallum.) Dill. MTusc. t. $47 \cdot \sqrt{i g} \cdot 37$.

In sylvaticis ad aggeres pinetorum, in humi. dis, ad latera fossariun; Vere, Autumuo.

30. B. fragile, capsulis erectis ovatis, setis Alexuosis, foliis lineari-capillaceis subsecundis. Dicks. Frasc. 3. 5. Dill. Musc. t. 47. fig. 53. F. G.

In rupestribus sylvaticis prope Erlangam collegi : Vere. (In statu sicco vaide fragile, operculum acutum obliquun; an diversa species a Br. flexuoso? -)

51. n. flexuosum, capsulis operculisque erectis, foliis setaceis, pedmuculis nexuosis. L. Dill. Musc. t. $47 \cdot \sqrt{\iota} \mathrm{g} .33$.

In sylvaticis (prope Giessam Dillenius - Calt. libus altioribus, operculis erectis praecipue a prio. $r i$, an coustauter diversum ? - )

*52. B. glaucum, capsulis cernuis, foliis erectiusculis imbricatis concaris, ragilibus, caule ramoso. Io Dill. ATusc. $t .46 . j \mathrm{~g} .20$.

In ericetis sterilibus, sylvosis; Vere.

53. x. spurium, capsulis cermuis, foliis nitidis acute lanceolatis concavis, in summitate 
mitate confertioribus patulis. Hedw. Musc. 2. $t .50$. (Dicranum sparium.)

In sylvaticis arenosis (Megap. primus detexit Tinrn.) Majo, Imuio.

34. B. longifolium, capsulis obliquis; foliis e lanceolata concava basi longe canaliculatim capillaribus, arcuatim heteromallis. Hedw. Mrusc.3. 6.9. (Dicramum longi folium Ehrh. crypt. exs. 114.) Dicks. fasc. 3. 7 .

Dubitauter adduco plantam Gottingae et in Hercyu. montosis absque capsulis lectam. Eandem vero Hallerus N. 1778. Hist. suae indicasse, specimina ejus manu in Helvetia lecta, evin. cunit.)

* 35. B. scoparium, capsulis erectiusculis. cernuis; pedunculis nggregatis, foliis secundis recurvatis, caule declinato L. Dill. Musc. t. 46. fig. 36. Fl. lond.

In sylvaticis, ad radices arborum vilgare; Vere.

36. B. rugosum, capsulis ovatis cernuis; foliis lanceolatis apice serratis rugoso-nndulatis subsecundis. Ehrh. pl. crypt. exs. 271. (Dicranum undulatum.)

In uliginosis mrfosis (Hercyn. Weber, Schra. der spicil. 59.) Vere. (Variis Germaniac locic promiscue chm priori collectum habeo, a quo differt: foliis latioribus lanccolatis patulis apice argute serratis, rugoso nudulatis, caule tomen. to denso ferrugineo obducto.?

Den- 
Dentibus 52 integris, vel capillaribus crectis, per paria approximatis, basi

\section{connatis.}

*57. B. undulatum, capsulis erectinsculis, pedunculis subsolitariis, foliis lanceolatis! carinatis mndnatis paremibus serratis L.

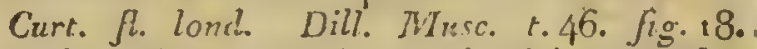
Hedw. Musc. 1. t. 16. (Polytrichinm undu-. latum.)

In syiraticis linmidis ubique: Vere - Angu-. sto. (P. jumiperifolinn, capsinlis foliisque angne. stioribus erectiusculis, caule simplici. Dill. Musc. tab. 46. Jig. 19. Hediw. Musc. 1. tab. 17.)

38. 33. rigidulum capsulis ovato - oblongis: operculo subulato currulo, foliis lan-. ceolatis erectis patulis, $e$ vasormm fasciculo onspidatis rigidniis siccitate tortilibus, can-. le erecto superne ramoso. Hediv. Musc. 5. e. 4. (Didymodon rigidnlum.)

In saxis, rupibus, interstitiis muri (I.ips. TTedwig. Gült. Schtrader: Trichostomıın angusta tum.) Alitumano, Vere.

53. 3. heterostichum, capsulis oblongis, foliis lanceolatis, in apicem canum denticulatum productis, nierescentihus. Hedre. MTusc. 2. 1. 25. (Trichostomum heterosticlinim.)

In montosis, ad saxa frequens; in sylvis supre lapides (Mlegap. Timm. Chemuitz Medwig.) Aestakc. (Br. fastigialum; catule ramo:o: ramis fastigistis, foliis lanceolatis in apicem cantum denti- 
denticulatnm productis Ravescenti viridulis, ca. panlis ovatis : operculis bieviter subulatis. Dill. Musc. Lab. 47. Jig. 50.?)

40. в. canescens, capsulis oblongo-subulatis: operculo longissimo aciformi, foliis lanceolato-carinatis enerviis, in apicem c2num argute denticularum productis $\mathrm{ha}^{-}$ rescenti - viridulis. Dill. Musc. tab. 47. fig. 27. Hedw. Musc. 3. tab.3. (Trichostomum cancsceus.)

In ericetis, sterilibus vulgare; Autumno, Vee re. (V)ariat surculis crassioribus, foliis confertis recurvis Dill. l. c. E.)

*41. B. elongatum, canle elongato superne ramoso: ramulis alternis pinuatis, foliis confertis recurvis in apicem canum denticulatum brevem prodnctis. Dill. Muse. tab. 47. fig. 31.

In ericetis montosis; Vere. (Trichostomum elongalum Ehrth. pl. crypt. exs. 233. Trichost: ericoides Schrader spicil.62.)

42. B. lanuginosum, caule procumbente, ramis lateralibus alternis fertilibus, foliis lineari-lanceolatis in apicem conum longum eroso - denticulatum productis. Hedw. Musc. 3. tab. 2. (Trichostomnm lanuginocum.) Dill. Muse. tab.47. fig. 32.

In sylvis montosis, inter saxa, ad terram: Aulumno, Verc. ('Trichostomum ramigerum Timm. Megap. 215. Trichost. serratum $E h \cdot h_{2}$. pl. cropt. exs. 94. - Spec. 2 . 4. sub Bryo hyp. noide 
noide Lin. militant; quoad lubitum ab Hypuis differunt: vel calle erecto, rel seta in ramis (crminali.)

* 45. B. microcarpon, canle ramosn: ramis fastigiatis elnugutis fertilibus, foliis lanceolatis cariuatis nerro mediu valıdo in apicem caum luevem denticulatum producto, capsula nvata miunt. Dill. Musc. tab.47. fig. 29 .

In montosis (Hcrcyn.) promiscue cum sequen. i: Antumno ("iolia superins in ranis viresunt, infente $\mathrm{ex}$ fuceo digricaut, set.e perbreves. Br. microcanpon, suremlo rano:n, foliis patulis, se. tis rerminalibuts, capsulis subrotundis Cimel. Sy'st. Nax. 235z.)

* 44. B. fasciculare, canle procumbente, raumlis confertis hrevibus, foluis lanceolaris margine revoluto patulis, capsulis ovato-ohlougis. Dill. Musc. rah. 47. fig. 28.

In nemosis ad saxa (Bructeri Heriyn.) Au. tumno, Vere ('Irichostomun lasciculare Jikra. der, spicil.61.)

* 45. B. capillaceum, capsulis erectis nblongis pedurulis terminalihus, Inliis setaceis patulis decurrentihns. Dicks, fasc. 1. 4. c. 1. fig. 6. Hedwir Musc. 2. tab. 26. (Swarria capillacea.)

In palustribus muntosis (prope Nicder-Sachs-

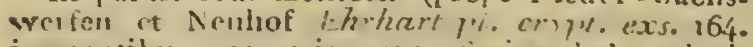
in montibus gypsaceis prop" seciecreal, ad ripas fluvii Bode pone Blunkenhury schrader spicil. 6.t. Didymodun capillactum.) 


\section{(suscl.)}

46. в. Didymodon, capsulis erectis oblongis, foliis ex uvato-concava basi su. bulatis. Hedw. 1. t. 27. (Tricliostomum pusillum.)

In arenoso-lutosis apricis (Lipsiae Hedwig: Berol. Willd. Megap. Timm. Ditrichum pusil. lum.) Autumno.

47. 13. pallidum, capsulis urceolatis erecris, pectine spirali, foliis capillaceis, surculis acaulibus. Schreber spicil. 103g. Hedw. Musc. 1. t. 27. (Trichostomum pallidım.)

Ad latera fossarum aggerumgue (Lips. Sc7ereber Dresdae IIediv. Beiol. Wilid.) Majo, Inl. (Specimina Halleri hist. 1805 eandem indigitant plautam. Similem habco ex America septentrionali, ex anreo pallidam, dentibus peristomii ad basin fissis.)

Dentibus capillaribus, spiraliter convolutis.

48. $n$ convolutum, capsulis erectis olılorigis, foliis lancenlatis, perigonialibus incyIindrum convolutis nbtusis. Hediv. Musc. 1. t. 32. (Barbula convuluta.)

In ericetis arenosis; Vere. (Br. convolutum Dicks. hort. sicc. brilant. fasc. 1. 20.)

40. n. setaceum, capsulis ectis oblongosubulatis: operculis filutmmilus longiundine fere capsulurum. J. (Mnium seta: enm.)

Ad muros et aygeres; Vere. (A priori differt: foliis ovato lanceolatis acmminati, raule inmo. vationibus ramoso, foliis jerigonialibus lanceolatis, operculis longissimis temissimis.)

D) 
*50. B. mucromulatum, capsulis crectis ob. longis, surculis erectis subsimplicibus, foliis lanceolatis carinatis mucronatis in humido patulis. Dicks. fasc. 5.5.

In ericclis, muris, ad vias frequens vere. ( $\mathbf{E}$ longiuquo Br. purpureo simile. Var. caule cras. siori, scta breviori Hedu. Musc. 2. ใ. 23. Bar bula unguiculata.)

*51. 3. cirratum, capsulis ovato-oblgrigis: operculis acuminatis, foliis congestis lancenlatis patulis, capillaribus, siccitate convolutis. Dill. Musc. 1.48 . fig. 42.

In muris, tcclis, ad aggeres sepes; Vere.

*52. 13. fallax, capsulis oblongis, opercitlo oblique rostrito, foliis e lata basi lar:ceolatis cirinatis recurvatopatulis, in siccir tate convolutis, canle innovationibns ramo. so. Hedu. IMusc. 1. t.24. (Barbula fallax.)

Ad muros, in ambulacris (Lipsiae Hedw.) Autumno, Vére. (Folia huuucctata statim recur. va, patula.)

53. ж. nervcsum, capsulis oblongis superinis paulo angustioribus, operculo subulato subobliquo, foliis erectis imbricatis lanceolatis nerrosis: nervo medin crasso fuscidulo, canle subramoso: ramis incrassatis. Dill. Musc. t. 48. fig. 47 .

In muris ad icrram; Vere. (A Bryo mucronulato differt: caulc longiori superuc ramoso, foliis rigidioribus, densioribus, nervo medio crasso fusco, in peculiarem mucronem non producto, setis 
setis brevioribus, capsulis longioribus. Var. fo. liis et capsulis lonfioribus. Vix satis a B. ner. voso diversum: Br. umguiculatum Dicks. foliis et caule brevioribus hort! sicc. Uritarm. fasc. 6. 81.)

54. B. barbatum, foliis lanceolatis acutis: summis patulis, capsulis erectis oblongis: operculo acuminato obliquo, pedunculo laterali. Curt. Jl. lond. Dill. Musc. tab. 48. fig. 48 .

In muris; autumno, Vere (Var. Br. unguicu: lati Schreb. spicil. 1037.)

55. в. rurale, capsulis erectis cylindricis, foliis piliferis recurvatis, canle erecto Iamoso. L. Dill. Musc. tab. 45. fig. 12.

In tectis, muris, agris, vulgare; Vere.

56. B. murale, capsulis erectis oblongis, folits piliferis evectis, canle brevissimo suhdiviso. L. Dill. Musc. t. 45 . Jig. 14.

In muris, tecris vulgare; Vere. (Hujus loci Barbula hercynica schrcad. spicil. 65.)

57. B. cuneifolium, capsulis erectis cylin . dricis, surculis subacaulibus, foliis cunei. formi - ovatis reticulatis pellncidis. Dicks. fasc. 3. 7. Dill. Musc. t.45. fig. 5.

In arenosis hinc inde cum priori (schreb. spi. cil. 747.) Vere.

58. B. stellatum, capsulis oblongis erectis, foliis patentibus maraine involntis. D 2 caule 
canle exiguo. Schreb. spicil. 10.4n. Hedu. Musc. 1. tab. 25. (Barbula rigida.) Dill. Musc. t. 49. fig. 55.

Iu muris e limo constructis (lips. Scherelier, Hedwig. Hildes, Schrader.) Hannoverae Ehr.h. pl. crypt. exs. 34 . Br. rigidum Huds.) Inu. Sept.

* 59. ז. suluulatum, capsulis subulatis; foliis Lanceolato - concavis acutis, caule exiguo. I. Dill. Musc. t. 45. Jig. 10. Curtis Flor. lond.

In subhumidis umbrosis frequens; Vere.

6o. I. tortuosum, capsulis erectis, foliis setaceis inermibus arefactione retortis cincimnatis I. Hall. hist. 6. 45. Jig.2. Dill. Musc. . 48. fig. 40.

In noutosis, rupestribtrs, subalpinis (Nassor. Leers. Tormla tormosa. Hamuverae Ehrz. pl. crypte. exs. 20.4. Palat. Tyrol. cic.) Autumno, Ve: re. (Nec linearia, nec serrata sunt folia.)

\section{MNIUAT (Sternmoos) Gl. pl. 1649- 1654 .}

Peristomium duplex: exterius dentibus 16 , interius membranaceum, lacinulatum. Calyptraglabra: flos niasculus femiueusque rermiualis. Caulis erectus.

* 1. M. androgyzum, caule ramoso vel sim. plici, foliss lanceolatis, masculis capitulis nudis globosis. L. Dill. Musc. 1.31 . fig. 2. Schunid. unal. t.56. fig. 1. 
In sylvaticis umbrosis, ad radices arbarum; Vere.

* 2. M. palustre, caule dichotomo, foliis lanceolato - subulatis carinatis. L. Dill. Musc. t. 51. fig. 5. Sclmid. anal. t. 56. fig. 2 .

In pratis paludosis; Vere, Aestate.

*. M. squarrosum, caule ferrugineo tonentoso, folis quinquefariis imbricatis recurvis, capsulis oblinguis. I.

In pratis nliginosis (Hinter Möllen Ducat. Iirunsvic. Luneb. Tifurturt. IVIegap. Timm.) (Canlis superne elougatus vel ramosus, folia per lentern argute sermiata; seta longa imnovationibus iuferior, capsula erectiuscula obliquua,

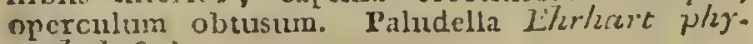
coppl.yl. 69.)

* 4. M. triquetrum, foliis trifaris patulis subulato - lanceolatis carinatis, capsulis obliquis. L. Hedw. Musc. 1. 21, 22. (Mee. sia lougiseta.)

In turfosis paludosis; Vere, Aestate. (Peri. stomii dentes exteriores, 26 , breves, obtusi ; in. teriores per paria approximati. Tristichis Ehrh. $\gamma / 2 y(0 j / 2) \cdot$. 59.$)$

* 5. M. trichodes, foliis lineari-lingulatis obtusis, capsulis obliquis. L. (B2. trichodes.) Hedw. M/2usc. 1. . 1, 2. (Meesia uliginosa.) Dill. Musc. t.49. fig.58.

In pratis paludosis, turfosis; Vere, Aestate. (Peristomii dentes extcrinres 16, breves, obtusi; interiotes reticulato. Laciniati.?

6. MI. 
6. M. Pohlia, folitis lineari-lanceolatis, capsulis obligue erectiusculis basi elongais. Hodw. Musc. 1. t.56. (Pohlia elongata) Br. elougatum Dicks. fase. ml. crypt.2.3.

In sylvaticis ad vias cavas (Chemnitzii in $\mathrm{Sa}$ xon. Hedue. Hannov. Ehr.h. jl. cryli, exs. 64.) Vere, Acstate. (Capss. ov sto olloniza, apoplly si quasi obconicae dugusliori iusidens. P'rristumii dentes exteriores 16; interioris membrana sede. cimpartita.)

*. M. hornum, cünle simplici, follis mirgine acute serratis, capsulis cylundricis pendulis. 1. Dill. Musc. t.51. fig.71.

In nemorosis udis; Vere.

*. M. crudum, caule simplici, foliis ovato Ianceolatis, summis elongato - linearibus apice denticulatis, capsilis pandulis. I. Hedu. Musc. 1. t.57. Dill. MIusc. t.51. fie. 70 .

In nemurosis umbrosis; Vere.

9. M. Gimum, caule mbiginosn, foliis lanceolatis alternis, capsulus pendulis pyriformibus obursis. Schreb. spicil. 1047. (Br. bimum.) Dill. Musc. t.51. Jig.73.

In pratis palndosis; Vere (Br. triquetrum leers flor. herb. 826. var. B. Foliis acntioribus apice subscratis, magis quam capwulis a sequen. (i differt.) 
* 20. M. p feudotriquetrum, foliis ovato. inceolatis acutis patentibus, capsulis oblongo - ovatis pendulis. Hedw. Musc. 3. t. 7. Dill. Musc. 1.51. fig.72.

In paludosis, apricis udis; Vere. (quoad Dillcu. citatam figuram Br. veutricosurn Dicks. firsc. 1. 4. lutus loci, at in differentia specifica folia stubulato - lanceolata dicuntur.)

* 11. Mr. turbinatum, foliis rubentibus ovato-lanceolatis patulis, capsulis pyriformi. bus pendulis. Hedw. Musc.5. t.8. Dill. Musc. 2.51. fig. 74.

In arenosis udis (vallis Zschopaviensis Fled. zuig.) Vere. (Truncus statura et dimensioute con. stailter inferiur praccedeutis speciei, codem vero colore fircatus. rredw. Nervus cum reticulo foliorum inferiorum rubens. Capsula constanter pendula pyrilormis ; operculum papillatum.)

* 12. M. annotinum, foliis ovatis acuminatis pellucidis, pedunculis subradicalibus; capsulis uutantibus. L. Dill. Miusc. t. 50. fig. 68.

Locis udis, sylvaticis; Inu. (quoad folia Juthjus loci: Mn. ramosum L. iMn. prolixum Neck. meith. 255.)

*13. M. nutans, foliis lanceolatis subulatis, capsulis oblongis nutantibus. Hedw. Musc. 1. t.4. (Webera nutaus). Br. nutans Schreb. spicil. 1043. Dill. Musc. 1. t.50. fig. 61 . 
In sylvis abiegnis, solo arenoso; Vere (Exemplaria novis e trunculo emissis ramificanionibus, quales Dillerius 1. c. dedis, Timmio nosiro debeo.)

*4. Mr. pyriforme, canle simplicissimn, foliis perichactii capillaribus Inngissimis recurvis, capsula pyrifnimi. I.. Hedw. Musc. 1. t. 3. (Vebera pyriformis.) Dill. Musc. เ. 5o. fig. 6o.

Iocis lapidosn sabulosis, seplentrionem ver. ixaliter spectantibus; 111 fissuris rupium, interstinis muronm ; vere. (Br. jendulum E/ur/. pl. cropur. exs. 165.)

* 15. M. caespititium, foliis lanceolatis acu minatn - sctacris, capsulis pendulis pendunculis longissimis. 1 . (Br. caespititimu.) Dill. Musc. $t .50$. Jig. 66.

In muris, siccis suerilibus graminosis fre. quens; Vere. SVar. capitulis el scrix breviori. bus, foliis longe capillaceis, locis siccis. Dill. l. c. E. F.

* 16. M. camillare, fnliis ovatis setiferis carinatis, capsulis pendulis, pendunculis longis. L. Dill. Musc. . 50 . fig. 67 .

Ad muros, in nliginosis; Vere.

*17. M. hygrometricum, fnliis oblongis concavis acuminaris conniventibus, capsulis obliquis prifomibus, pedunculis arcuatis. L. Dill. Musc. t.52. fig. 75 . 
In humidis, umbrosis, ad sepes, muros; Vere. (Peri-romii dentrs obliqui apice cohaerentes: Koelientera lledw. find. 2. 95. Funaria Schseb. Gen. pl. 2650.)

* 18. M. argenterum, foliis ovatis concavis imbricatis connventhus setiferis. smronlis teretibus, capsulis pendulis. 1. Dill. Musc. t. 5n. fig.62. Flor. dan. 1.88 n.

Ad terram sabulosam, in muris; Aunumno, Hieme. (VI. holasericemn, folii- ovatis concavis inblicatis comniventibus acuuninatis, surculis anuntioribus, capsulis pendulis. Dill. MTusc. 6. 50. fig. 63.)

19. M. carneum, foliis lancenlatis alteruis pellucidis, capsulis nvatis pendulis. L.
Hedu. MTusc. 1. t.20. (Br. delicatulum.) Dill. Murc. t. 5 o. fig. Fn.

Lnciu humidis, umbrosis, ad fossulas pratonum; Vere.

20. M. stellare, foliis ovatis aristatis serratis, pedunculis cauleque simplicilus, capsnlis nutantibus. Timm flor. megap. 846 . Dill. Musc 0.52. fig. 78 .

In umbrosis, ad vias cavas ; Vere. (Specimi. na ab ipso Timmio accepra folia ostenduut per lentem scrrata. Hujus ulam loci esse: Br. serratum, surculis simpliribus, folii- ovato - acutis: summis augustioribus serratis, thecis ovatio nutaulibun, operculi roarello (in juniori platuta ut jam Dillemius 1. c. annotaverat) recurvo sichre. der gicit. -1 - evincmut peristomii interioris similis fabrica: emembrauacea carinata divisa 
margine in lacinias pyramidales reticulato-den: ticulatas apice quasi erosas - et viva Allctoris exemplaria.)

* 21. M. cuspiclatum, foliis ovatis acutis scrratis, surculis reptantibus, caule sim. plici, pedunculis ageregatis vel simplicifus, capsulis nutantibus. Schreb. spicil. 1n49. (Br. cuspidatum.) Dill. Musc. $t$. 53. $\operatorname{sig} \cdot 79$.

Locis umbrosis, Vere, Aestate.

* 22. M. serpillifolium, foliis oboratis obtusis integenimis, pedunculis aggregatis vel simplicibus, capsulis ovatis cernuis. $L$. Dill. Miusc. 0.53 . Sig. 81. Sclureb. spicil. 755. (Br. punctatunı.)

In graminosis, umbrosis, sylvaticis; Vere, setate.

25. N. ellipticum, foliis oblongis ellipticis subserratis apice obtusis emargiutis mucronulatis, pedunculis aggregatis vel. simplicibus, capsulis oblongis cemuis, operculis cuspidatis. Dill. Musc. 4.55 . fig. 80.

Hinc inde cum priori; Vere. - An luxins loci :

24. n. rosiratum, surculo simplici, foliis ovato-ligulatis subserratis, tlecis ovaris nutatibus: operculi rostello recurvo. Schrad. spicil. $7^{2}$. 
In sylvaticis rupestribus; Vere, Aestate. (Fo. lia ut in Mu. serpillifolio; per leutem maxime allgentem subserrata, obtusa cum urevi mit. crone.)

* 25. M. roseum, folits rosacee congestis lincenlatis acutis denticulatis, caulibus sim. plicibus vel proliferis, pedumculis arruregaris, capsulis nutantibus. Sihreb. spicil. 10,8. (Bt. 1osenm.) Dill. Musc. t. 52. fig. 77 .

Locis udis umbrosis; Vere, Aestate.

*26. M. undiulatum, foliis oblongis undulatis serratis, pedunculis agoreratis, capsulis nulautibus. T... Schreb. spicil. 753. (Bı. ligulatum.) Dill. MTusc. $4.5 \%$. fig. 76 .

In nemoro-is, pomariis, ad sepes; Vere. (Inter praecedentia speciosissimum.)

27. м. Timmia, foliis lineari-lanceolatis serratis patulis, capsula cermua, oper. culo convexo ceutro depresso. Hedw. Murc. 1. t.52. (Timmia megapolitanı.)

In pratis turfoso-paludosis (prope oppidum Malchin Ducat. hiegapolitaui Timm.) Majo. (Pe. ristomii interioris laciuiae articulatae appendi. culatae perforatae, apice conjugatae. Elegan. tem plantulam unn solum oculatis:imo Timmio deuro, sed eandem ex America septentr. a $D$. Mühllenberg accepi).

28. м. marchicum, foliis nvato-lanceolatis integris, caule erectiusculo suporue ramoso, 
moso, capsulis subrotundis obliquis. Hudw. MTusc. 2. t.39. Willd. flor. berol. 944. t.6. fig. 12. (Leskia marchica.)

In ligno putrido natante (prope Spandar, Willdenow) Majo, Iunio. (Peristomii interni Iaciniac bifidae.)

*29. 11. fontanum, foliis Inceolato su. bulatis subsecundis apice denticulatis, caule simplici genicnlis inflexo superne ramnso, capsulis subrotundis obliquis. L. Dill. Misc. t. 44. Ji.g. 2.

In pratis ndis, nliginosis; Vere. (Peristominm interum inter columunlas simplices, e dorso carinarum eminentes, tencrime ciliatum.)

5o. M. laterale, foliis lasceolato-setaceis subsecundis denticulatis, cansulis cratis pedumculatis lateralilus. Huds. angl. 2. 485. (Br. laterale.) Hedw. Musc. 2. t. 40. (Bar. tramia lialleriana.) Hall. Hist. t. 46. fig. 8 .

In montosis eylvaticis supra scopulos (Saxon. Hedw. Benmeartell. Hercyn. Ehrh. $h l$. cropt. exs. 53. Carinth. Whulfen: Br. recuremon Jracg. Coll. 2. 224.) Vere. (Ecristomii externi dentes 16, subulati, interius plicato - carinatum lacinu. latum.)

* 31. M. pomiforme, foliis lanceolato-seta. ceis patulis, denticulatis, capsulis splraericis erectis. 1.. ( $\mathrm{Br}$. pomiforme.) Dill. Musc. t. 44. Jig. 1 . 
In umbros's, cavermulis rupium ; Vere. (Pcristomii dentes ut in puiori. Folia lentius a su. bulata basi in setaceum acumen contrahustur. Insignem varictatem ex alpinis Tyrolis haleo, foliis multo brevioribus, cauliculis angustiori. bus et longioribns, inferne rubiginoso tomento obrolutis. Capsulac laterales, sed longe pedun; culatac. Intermedia quasi species inter hauc ct an: tccedcutem.

\section{IrYPNUM (Astmoos) G. pl. 1656. 2658.}

Peristomii dentes simplici duplicive or. dine; flos masculus feminensque axillaris, gemmaceus. Caulis frondosus vel ramostas.

Fronde simplici, subramosa, pinnatr. s * 1. H. Tryoides, fronde simplici decum. bente pimuta lanceolata apice pedunctifera, capsulis erectis. L. Heclw. Musc. 3. t. 2g. (Fissidens bryoides.) Dill. Miuss. t. 34. fig. 1.

Locis umbrosis, cavis; Hieme, Vere.

* 2. H. taxifolium, fronde simplici decumbente pinnata lanceolata, basi pedunculife: ri.. L. Dill. Musc. t. 54. fig. 2.

In sylvaticis humidis, cavernulis ad terram; Autumno, Vere.

*3. H. adiantoides, fronde simplici vel subramosa piunata, medio pedunculifera. I. Hedis. 
Hedwe Musc.3. 1.26. (Fissillens adiantoidcs.) Dill. Musc. t.54. fig. J.

In paludosis prope aquas; Vere (Peristomii demes in spec. $1-3$. ordiue implici, 16 , bilidi. Folia H. adiant. jum per lentem modice augentem apicc serrulita.)

*4. 1. denticulatum, fronde simplici pinnata duplicata, basi pedunculifera. L. Dill. Musc. t. 34 . fig. 5. Vere.

In umbrosis ad tcrram, arborum radices;

* 5. H. sylvaticum, fronde subramosa pin. nata, supra basin pedunculifera. Huds. angl. 2. 419. Dill. Musc. t. 34. Jig. 1. Vere.

In umbrosis ad terram, radiccs arborum;

*. H. lucens, fronde subramosa pinnata inbricata, foliis rcticulatis planis, supra basiu pedunculifera. Lo Dill. Ausc. t. 34 . fig. 10 .

Locis nmbrosis, paludosis (Hercyn. Web. Hannov. in monte Dcister Lammersdorf. Ehr.

Frondibus ramosis pinnatis.

* 7. H. trichomanoides, fronde ramosa, fo. liis distichis imbricatis acinaciformibus obtusis. Schreb. spicil, 1053. Dill. Musc. t. 34 .
fig. 8 . 
Ad arbortum radices, in uemoribus nmbrosis; Vere. (Leskia trichomanoides $E: 72 \%$. cropte. exs. 25. Sequenti affunis, quocum ab Anctoribus confunditur.)

* 8. H. complanatum, fronde ramosa pirs. nata, foliis distichis imbricatis acinacifor. mibus acuminatis. L. Schreb. spicil. 778. Dili. Musc. c.34. fig. 37.

Ad arborum truncos frequens: Vere. (Surculi 3. ramuli in fila attenuati ; setae axillarcs breves longo perichaetio ciuctae; capsulae ovatac.)

9. H. pennaium, fronde ramosa pinnata, foliis bifariis lanceolatis resime acuminatis, capsula perichaetio immersa. I. (Fontinalis pennata. Hedw. Musc. 3. t. 1.9.) (Ne: ckera pennata.) Dill. Musc. t.32. fig. g. Hall. hist. t. 46 . fig. 2.

In arborum cortice (Silcs. Francon. Erlangae Kassov. Leeers Herb. S26. Hypu. undulcutun: fi. de cxemplarium 1. c. collectorum.) Vere. ( $\mathrm{C}$ ristomii dentes ordine duplici: alternatim breviores ciliares. Halleri et Dillenii fig. cit. hujus loci esse, evincuut prioris exemplaria ad Willichium missa. Neckera pumila Hedw. l. c. t. 20 . differt praccipuce seta perichaetio longiore.)

* 10. 11. undulatum, frondibus ramosis váge pinnatis, foliis undulatis complicats nitidis. L. Dill. IMusc. t.56. fig. 21.

In umbrosis sylvaticis montosis Hercyn. VC. re. (Peristomii dentes duplici ordine: exteriores 16 , integri ; interiores e membrana difformi ci. liati.) 
*21. 11. crifpum, froudibus ramosis pinmatus: foliis undularis plumis. L. Dill. Musc. c. 36 . fig. 12.

In sylvaticis saxosis, rupestribus, passim fre. quens; Autumus.

\section{Caulibus vel surculis ramosis pin- natis.}

* 12. H. purum, surculis pinmato - sparsis teretibus, folnis ovatis concavis mucuonatis comniventibus. L. Dill. Musc. t. 40. fig. 45. Curt. flor. Lond.

In pratis, pascuis, nemoribus, Verc. (Sctis prae. longis, surenlis pinatis actentatis ab H. illecebro distinguinus. Hujus loci Hypn. clegans L/Lv/L. cropt. exs. 242.)

* 15. H. compressum, surculis plann - pinnatis apice attentutis, foliis ovaro-lancenlatis. Schreb. spicil. 1067. Dill. Miusc. t. 40. fig. 47.

In nemorosis declivibus; Hicme, Vere. (IIujus loci : Hypu. purum Lhih. cr.7\%. exs. 31. Hypu.

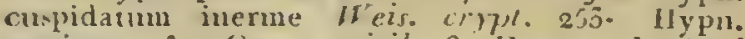
muicum vin Genns spicil. 76. Hypn. Schreberi Willd. 935 ; nou confundendum cum Hypu. com. presso lin.)

*14. H. cuspidatum, surculis pinuato-ramusis, apice convoltutis acuminatis. 1. Dill. Musc. t.5.g. fig. 3\%.

In pratis, pomariis hmidis, palndosis; Maj. Iun. (Folia iu hoc ct compresso chervia, canliua 
lina magis cordata, in illo ovata. Caulis in utro. yue purpurascens, compressus magis in priori, in hoc setac caule et surculis longiores. Var. canle simplici sparsim pinulato, fol. longioribus acutioribus.)

* 15. H. triquetrum, surculis pinnato-sparsis recurvis, foliis triangularibus patulis. L. Dill. Musc. t.38. fig. 28.

In sylvaticis, ad terram; Hieme, Vere.

* 16. I. nicens, surculis erectis brevissime pimato-sparsis, foliis lanceolatis subulatis striatis. Sichreb. spicil. 1062. Dill. Musc. c. $5 \mathrm{~g} \cdot \mathrm{fig} \cdot 37$.

In pratis pandosis; Vere. (Hypu. trichodes Neck. Grillob. 403. Perichaetia praelonga. Setae caule surculisque longiora. Capsulae inclinatae operculo conico brevi. Ab lioc differt capsulis erectis, nec cernuis, perichaetip brevissimo, sur. culis paucis elongatis: Hypn. refuscins ramis compressis, fol. pilo aristails uitentibus, capsulis crectis, operculis conicis Jicks. crojtt. 5. 9. 1. 3. Jig. 4. Hall. hist. 1784, quod ex alpinis Helvetiac habeo, ab ipso Hallero collectum.

* 17. H. abietinum, caule surculisque pinnatis remotis filiformibus superie incras:satis. 1. Dill. Musc. t.35. fig. 17. Vaill. Paris. $1.29 \cdot$ fig. 12.

In aridioribus, sylvis abietinis; absque ca. psulis. (Folia ovata, nervo medio robusto in acuimell fere producto, sicca adpressa, maxgiแลเฉ.) 
18. H. proliferum, surculis proliferis triplicato-pinnatis - capsulis incurvis, L. Dill. Musc. tab.35. Jig. 1.4. Curt. lond.

In sylvis, pratis bumidis, Vere. (Folia er: surculi virescuut abque nitore, caulinal ovato-. cordata, medio nexvo in acumen producto. Hujus ioci Hypu. delicatıun E/(r/. crj7u. exs. 301.) । Schrader. spicil. 73.

* 9. Ix. umbrarum, surculis proliferis bi-. pimuato - sparsis attennatis, capsulis obliquis. Ehrh. crypt. exs. 66.

In Hercyuiae umbrosis (Ehrh. l. c. Folia cordato ovala acuminta striata, margine per lell.. em serrata; setae vix unciales.)

20. H. parietinum, surculis proliferis plano - bipinuatis, capsulis obliquis. I. Dill. Musc. t. 55. fig. 15 . Schnid. Ic. t. 58. fig. 5 .

In sylvis ad serram; Vere. (Surculi pellucidi mbent; folia majora, splendentia eucrvia, ova. to acumtuata. Hujus loci Hypn. proliferum Ehrh. crapl. cxs. 95. Hypur. splenulens, sur. culis subriplicato - pinnatis proliferis, foliis lan. ccolatis confertis plendentibus, podumculis aggregatis. Sibth. Flor. oxon. 304.)

\section{Foliis secundis vel reflexis.}

* 21. H. commutatum, caule procumbente. surculis amoso - pinmatis scuferis, foliis 
secundis. Hedw. Musc. 4. 6.26. Vaill, par. t. $29 . \sqrt{i g} \cdot 9$.

In palustribus, ad rivulos; Aestate. (Hujus loci: Hypu. Jilicinum ramoso - pinnatum $W$ tis crjpe. 229. Dill. Jig. 22. tab. 36. formam non male exprimeret, quz:e vero ab Hedwigio ad Hypı. sum molluscum referur. Setae ex ra. mis foliorum, nervus apicem non attingens, catı. lins neıvoso - striata uncinata.)

* 22. 1r. affine, caule procumbento ramo. so-pinnato setifero, foliis subsecundis ner. vosis: nervo ad apicem ducto. Dill. c. 36 . fig. 19.1 .

In humidis, palndosis. (Differt a priort cum foliorum figura et magnitudine, tum horum Eil. sciculo ducinlornm exura paginae apicem emer. geute apiculi facta; frucu semper truncos de. cumbesites occupante, neque ramos proceriores surrectiusculos fleálv. l. c. Fold cauliua et ramea subsecunda usque ad apicem nervosa, re. ctiora e latiori basi lunceolita; perichaetialia Janccolata acuminata nervosa.)

*23. 11. filicinum, caule erecto simplici pinnato setifero, folis nncinatis secumlis striatis. Dill. Musc. t.36. fig.19. B.F.

In humidis; Aestate. Canlem simplicem rel parmm ramustum erectiorem observo, folia lon. giora molliora per lentem striata, apiceque, praescrim caulina, obiter denticulatil. Foliula peri. gonialia interiora longissine apiculata lanceo. lato - subulata nervoso-striata, capsulae incurvae. Perist. dentes exteriorts integri, interio. E 2 
res membrana striata connexi intermedis cilis. Operculum breve. Inujus Ioci: Hypu. Crista ca. otrensis Ehr/e. (rypt. exs. 5.)

2.1. 11. crista castrensis, smculis pinua* tis: ramulis apjoroximatis, apiciluus recur. vis. L. Dill. NLusc. t. $56 . \int_{\mathrm{g} g} .20$.

In sylvis ad radices rupium; Autummo; Vere. (Folia secunda cincinuta ad leutem encrvia. liuius loci: Hypuma molluscutr, procumbeus, ramis sinplicibus curvatis, foliis coufertis varie distortis iucurvis, duculormn fasciculo destitutis. Hedw. MIusc. 4. 1.22.)

* 25. H. extricatum, caule decumbente setifero, folis uncinatis, smenlis erectis pinmatis sensim attenuatis apice convoluto - secundis.

In humidis uliginosis. (Surculi decrescendo pinnati, clian :iuplices. Folia caulina in luxmito acque ac sicco sccumda, caulina patula ma. gis, lacte virescentia, instructa nervo valido mil cuspidem extenso; per maryiues etiam subtilissime denticulata; perizonialia ovali lanceola. ta apice sacpe mubinda striato - nervosa. Ca. psula incurva. Operculum breve conicum api. culatum. Peri-t. cxterioris dentes 16 , denticu. lati; interioris membrana reticulata comuexi ab+quc ciliis.)

26. .. uncinntum, canle prostrato, foliis subulato-carinatis striatis, freteromalle um. cinatis, pericllactiis gracilibus lono is. Hedu. Musc. 4. 1. 25. 
Locis montosis (ex subalpinis Tyrol. habeo. Saxoll. circa Chemnitzinm, Tschopau Hedw.) Inu.

* 27. 11. cupressiforme, caule et, surculis vage pinnatis, foliis ovato-acuminatis inculvatis secundis enerviis. L. Dill. Musc. t.57. fig. 23. Hedw. Musc.4. t.23.

Ad terram, arborum trunros ct saxa; Vere. (Hypu. extenuntum, canle et surculis filiformimis, foliis ovato. acuminatis incurvatis secum. dis enervits, isill. l. c. fig. P, D, C. Hypu. lacuresarn, caule et surculi, incrassatis, foliis ovato. acuminatis incurvatis secundis enerviis lacmosis. Dill. L. c. fig. 2 ' C. Colnre ex viridi vel fusco - rulescente nitenri, foliis lacmo. ais crascioribus a cupressiformi, sctarum fre. quentia alisque notis a secuenti differt.)

* 28. H. rugosum, caule et surculis vage pinnatis exectinsculis incrassatis recurvis, fo! iis secundis recurvatis basi rugosis. 1 . Dill. Misc. $t .37$. fig. 24. $A, B$. Ehrh. crypt. ex.s. $2 g 1$.

Locis sylvaticis montosis ; Absque capsulis. (A priori differt foliis ductulostun fasciculo instructis ver-ns summitatem evanescente (Hedw. Musc. 1. ケ.23. A.) apicibus longioribus produ. ctis (emuistime sermalas.)

2n. 1t. scorpioides, ramis vagis procumbenibus apice incrassatis complicatis incurvis, foliis ovatis concevis secundis. T.. Li 3

D. 
Dill. Muse. t. 57. fig. 25. Dicks. hort. sice. brit. fasc. 3. 22.

Locis puludosis turfosis (Megap. Timm. H. squarrosum, crrore typograplii Flor. megap. No. 926. Finssen Nullerer -) Alseque capsitis. (Ex maximis quoad cunles, et folia, medio calt. Ic ovata concava mervia, ad lentem epice lire. vissime mucroulata s. apiculata, sunima ctiam acuminata, pesigouialia interiora lanceolata acu. minara. Sctae fongac, capsulac incurvac.)

5o. H. palustre, caule decumbente repen. te, surculis confertis erectiusculis sctis brevioribus, foliis nvato - acutis concavis secundis. L. Dill. ITusc. t.37. fig. 27. Hedw. MInsc. 4. t. 1. (Ieskea paludosa.)

Locis paludosis, aquosis; Aestate. (Perichactia plermnute ex caulc provenimut. Folia sicca hecromalla, ad lentem inedio nervosa. Capsislae erectinsculae: perist. ext. 16 dent.; inter. nembratuaceun 16 partium.)

* 51. H. aduncum, caule erectiusculo ramoso-pinuato, suculis patulis recurvis, folits oralo - acmminatis recmrvatis, foliolis perichatii apicnlatis. I. Dill. MTusc. 1.37. fig.26. Hedw. Musc. 4. t.24.

In paludosis: Acstatc. (Folia ductulorum fa. sciculo instrucis, circa extremitatcon cvanido. Var. sterilis sulmetsa, caulibus duplo sriplove lougioribus, lolids laxioribus.) 


\section{(MUSCI).}

32. H. Halleri, canle prostrato, surculis exectiusculis simplicrbus; foliis lanceolatis fasciculo destitutis, undique e basi imbri. cata recurvis. Heder. Musc. 4. t.21.

Ad fockstein Salisburgi Froclich - ex Alpi. nis Hielvetize habeo ah ipso Hallero collecka et Hist. suae 11. 17034. desigrata exemplaria - diver. sum, ab hoc foliis enerviis recurvis apice secune dis margine tennissime serrati , surculis ramoso - piniatis; est: Hypri. Halleri Timm. Fl. megap. 12.829 , 118 spccimina viva Auctoris docent.)

* 53. H. stellatum, caule execto, surculis sparsis; foliis ovatis longe mucronatis erecto- patulis. Schreb. spicil. 1061. Dill. Musc. t. 3g. fig. 35. Dicks. fasc. pl. crypt. 1. 5. t. 1. fbg. 7 .

In paludosis (pratorum Schoenfeldicorum Schreb.) turfosis Megay. Timm. Gotting. Heyder; Acstate. (Folia ex ovato trianmilla cuervia, longe mucronata patula, apicibins parum recurvis, in summitate canlis et surculornm stellatin expausa hutescentia. Foliola Perigonialia lanecolato subulata longissime mucronuta substriata. Setae longae. Capsula obliqua incurvil: operculnun breve conicum apicnlatum. Perist. exter. 16 dentatum, interins membrana. ceum venoso reticulntunn 16 partitum : ciliis interpositis geminis. Hlitus loci, nisi eadem spe. cies, ut exemplaria doceut Hallexi ad Willichinn missa: Hypuum foliis aptulis, subulatis . operculis conicis. Ifall. hist. 17ว̈\%.)

*3/p. H. squarrosum, surculis vagis erectiusculis, foliis complicatis ovato-lanceolaE if tis 
tis apice subulatis undique reflexis. $L$. Dill. Musc. t. 5\%. fis. 58 .

In subhumidis sylvaticis, pomariis: Autumuo Vere (Var. minor: Dill. l. c. /lg. 79.)

*35. rr. curtipendulum, surcilis vagis ramoso-pinnatis: loliis oratis acuminatis patulis, perichsetiis longissimis. L. Dill. Musc. t. 45 . fig. Gg.

Ad arborum truncos; Tere. (Surculi rel ra. mu'i apice incrassati stcllatim expansi. Scrac pe. ricaselio vix Inngiora. Capsulac pendulac de. mum ercciac. Folin ovatí nervo medio, apicem rersus longe productum serrulatum, evinisdo percursa : sicca subsccunda. Reckeria curripendula Tzmm. rnog. 11.801. Bubbilli s. gremmae in distinctis individuis contuent organa scxiss.)

36. H. loreum surculis vagis erectiusculis recurvis, foliis subsecundis. L. Dill. Musc. t. $59 \cdot$ fig. 40 .

- In sylvis ad terram; Verc. (Foliis basi ova. tis convexis striatis apice falcatis serratis, sld. tiun dignosciur. Var. minor: Hypu, heerero. phyllam, foliis caulinis squarrosis, surculorum ovatis acnminatis mudiquc serratis; capsulis ova. to oblongis.)

* 37. H. sciuroides. surculis erectis subramosis incurratis. L. Dill. Musc. t. 41. fig. 54. 


\section{(MUsci.)}

In sylvis, pomariis ad arborum trumcos. (Bul. billi s. gemmac in distinctis individnis continent organa sexus. Folia subsecund.ı, per lent. rem striata acmminala. Dicramun sciuroides Sibth. oxun. 280. Lihrh. crypt. oxs. 2s3.)

* 38. H. viticulosum, surculis erectis subramosis Bexuosis, foliis patulis. L. Dill: Musc. t. $3 \mathrm{~g}$. fig. 45 .

In sy!vis ad rupes, arborum truucos; Hicme. (Folia crispa subsccuida, in lumida planta pa. tula, per lentem flexura, apice linearia obtusa, nervo medio crasso. Neckera viticulosa Timm. meg. 12. 799.)

50. H. attenuatum, surculis ramosissimis, ramis incurvatis apice gracilescentibus incrassatisque, foliis ovato - acuminatis subsecundis. Schreb. spicil. 2073. Hedu. MTusc. 1. t. 12. (Leskia attentuata.) Dill. Musc. เ. $42 . \lg .66$.

Ad radices arbormm (Ting. Schreb. Chemni. tzii Tedu. in Comit. Pyrmont. Schrad.) In sylvi- supra lapides (licgay. Timm.) (Folia in hilmido patula e bsci ovata concava semiamplexicanlia, apice lanccolato - lincaria brevissime acumuiata, uervo medio valido evanido prope apiccun. Perist. ext. dent. 16 , intern. cilis 16 , basi tener rima comnexis.)

40. H. medium, surculis repentilus ra. mosis confertis, foliis lato-lanceolatis acutis subsecundis imbricaro-patulis, capsn-

$$
\text { E } 5 \text { lis }
$$


lis crectis. Dicks. fasc. crypt. 2, 12. Dill. Mruse. t. 42. Jig. 65.

Ad arbores (circa Giessam Dillen. Nassor. collectam accepi.); Vere. (Cauliculi repunt, un. s dique surculos incrissatos confertim et pinna. tim ramosos: rami: altenuatis varie, plerumule tamen rellexis, emitirutes; fclia orata, vel lato. lanceolata acuia, nervo medio ralido instructa, humida patula, sicca adpressa musecumda. Se? tac longinres surculis, cauliuae, foliolis perichae. tialibus lanceolato - subulatis aeque nervosis ciuctae; Capsulac erectae cylindricae; rarins obliquae vel mutantes. Operculum breve conicum.)

Foliis rectis, surculis fasciculatis, vel den:droidibus.

41. Ir. palatinum, caule rago, surculis ramoso-fasciculatis subsecundis, foliis orato-oblnmgis acuris, capsulis erectis oblongis. Neck. meth. 182. Dicks. fasc. crypt. 1. 6. Dill. Musc. t. 41. fig. 55 .

In arborum truncis, praesertim Fagis (Nassov. collectum habco - ctiam ad sasosa palat. Neck.) Autumno, Vere. (Surculi attenuati plus minusve incurri, folia in siccitate adpressa niteutia, per leutem encrvia, ex ovato acuminata integerrima, hine inde quasi luteo tincta colore. An hujus boci Hypu. gracile Linn. Eihrh.? saltem diversum quoad Gguram Hedwigii Misc. 4. $t .5$. Pterigyilandrum gracile.)

42. H. albicans, surculis erectinsculis, foliis ex orato longe acuminatis adpressis. capsu- 
cepsulis cernuis. Neck. meth. 180. Dill. Musc. t. 12. fig.63. Hodw. Muso. 4. t. 5 . Dicks, hort. sicc. brit. Jasc. 5. 21.

Iu arenoeis declivibus (Nassov. Francon. collectum labco Palat. Neck. Iips. Hedu.) Autumino, Verce (A priori differt: colore palli. diore, surculis minus densis ac ramosis, foliw majoribus ex ovato. Jougius acuminatis ad lertem tribus servis, modio validiors, polle apicem evanescentibus, iustructis, capsulis cer. muis, operculis apiculatis.)

*43. 11. "myosuroides, surculis erectis, ramis fasciculatis attonatis incurvis, foliis ovatis, capsulis erectis. Hediv. Musc. 4. t. 8. Dill. Musc. t. 41 . fig. 50 .

In sylvaticis ad radices arbornm, rupinm; Verc. (Folia ad lentem ovata, concava acuta, pallido nervo medio instructa, apicen non attingente. Occurrum specimiua foliis actutioribus enervii , capsulis obliquis, oralibus potins quam ovatis Hujus loci hypn. myosurum. Jizn. Dill, l. c. Jig. 51.? Hypu. viviparm Nech. Fhrh. crojt. exs. 57. Leskia vivipara Timm. Habeo crassiora halleriana cxemplaria hujus Hypni sub inomine H. illecebri ad Willichium missa, nec nou alia sub nomiue illecebri collecta plane Hypu. puro aemula.)

*44. H. dendroides, caule erccto simplici, ramis fasciculatis terminalibus simpliciu. sculis; capsulis erectis. L. Dill. Musc. t. 40. fig. 48. 
In pratis sylvaticis, pomariis; Autumno, Ve: re. (Folia cimlina adpressa, ramea striato.ner. vofj, apice serrata.)

* 45.-H. alonecurum, canle evecto simplici, ram s fascuculatis terminalibus subdivisis, capsulis subuntantibus. L. Dill. Musc. t 4 . fig. 49 .

Iu $11 \mathrm{cmorosis}$ truncos arbonm nipesque opericus; Autunuo. (Folia camlina patula, ramea. mervosa subserrata.)

Foliis rectis. surculis intricatis vel vagis.

46. H. polycarpon, caule et surculis de. cumbentihus filiformibus ramosis, follis adpressis nerrosis, capsulis erectis. Ehrh. crypt. exs. 96 . (T.eskia polycaipa.)

Ad terram et arbonum tmucos. Primn adspe. ctu grarilcscentihus vel incrassuts ramis, ctim incuivatis, similiudiuem habet cum Hypun at. tcmillo, a quo vero enpsularum cylindricarum multiudine. Canlibus et surculis decumbente bus, ramulis brevicimis, folits ovato . lances. lati-; a filifuruni vern nervo cormu crass? per medium usune ad apicem ducro, operoulo breviter rostratn, vel pntils conico, ueque 11013 peristomio duplici distiuguitur.)

* 4- . subtile, canle decumbente reptante: ramulis erectiusculis confertis, foliis exignis patulis, capsulis erertis. Hedu'. Musc. 4. t. g. (Leskea subulis.) 
Ad truncos arbornm (Gotzing. Schrad. Oc. currit vitriis Germ. locis.) (Folia in Inmido re. motiuscula, mudo oculo capillarsa, ad lamem lanceulato - acumiuata chervia, concava, va. ric plian curva. Capsuta oblonga erecta vel stubcruna: operculum conicum. Setae xamis duplo longioras.

48. 1. filiforme, caule surculisque fili* formibus, foliis adpressis concavis, capsulis erectis. Timm megap. 817. Hedw. Musc. 4. t. 7. (Pterigyuandrum filiforme.)

Iu saxis sylvarum (Megap. Timm.) Vere. (Fo. lia per lenteu ovalia concava enervia parum acuminata, imbricata ciiam lutuida. Capsulae cylindricae, operculum rostratmu, peristomium siuplex 16 dentatum.)

49. Ir. serpens, caule repente, surculis filiformibus simplicibus, subramasis, fo: liis lanceolatis acuminatis remoto patulis, capsulis oblongis ceruuis. 1. Dill. Musc. t. 42. Jig. 64. Hedw. Musc.4. t. 18.

Locis umbrosis, lumnidis; Vere, Autumuo: (Folia mudo oculo oblitterata, per lentem enervia, intcera, acuminata. Capsulae basi angustalite crectae, demum cernude; operculum bre. ve vix acutuun.)

50: H. dubium, caule subdecumbente, ramis adproxinutis filiformibus erectiusculis vagis subpinnatis., Loliis lanceolaris acutis crecto-patulis, capsulis oblongis in: curtis. 
curvis. Neck. meth. 161. Dicks. fasc. crypt. 5. 10. Dill. MTusc. t. 36. fig. 21 .

I:1 cricctis, paludosis (Priori statura majori. Folia per lentern integerrima margine, pelluci. da, creunlato reticulata, usque ad apicem lervosa, Lanceolata acuta vel acuminata, in liu.. zaido patula, in sicco erecta $\mathrm{rcl}$ subsecundi, apicem İmulorum rersus quasi in cuspiden convoluta. Setae longae glabrae. Capsulac cylindricae demum incurvae, operculum ureve; conico acutums.)

* 51. 11. praclongum, catule decumbente. surculis subpinnatis: ramulis remotis, fo. liis ovato-lanceolatis patentibus, operculis xostratis. L. Dill. Musc. t. 35. fig.15. Hedw. Muse. 4. E.2g.

In nmbrosis, humidis; Vere. (Fulia per lent. tem margiue serrata, nervosa. Setac exaspera. tac. Capsulac ovatae ceruuae.)

*52. 11. intricatum, surculis repentibus, ramis brevibus, foliis lanceolatis acuniuatis patulis, capsulis ventricosis obliquis cer-. nuis, operculo conico. Schrcl. spicil. 1072.

In corticibus arborum, ad trumcos cespitulis densis; Vere. (Folia margine ad lentem denti. culata, nervo valido versis apiccm evanescente percursa. Setae breves scabriusculac. Capsuliae: obliymae sub ammlo comtractae. Ammulus temuis fumbria composita; operculum conicum apice. apiculam brevem obscurioris coloris gerens. Var. setis longioribus; foliis et capsulis graciliori..
bus.) 
53. . velutinum, caule repente, surculis confertis erectis, foliis ovato-lancenlatis denticulatis, perichaetialibus interioribus crinitis, capsulis obliquis, setis exa. speratis. I. Dill. Musc. t. 42. fig. 61. Iedu. Musc. 4. t.27.

Ad arborcs, muros, aggeres; Vere. Folia lanceolata, uervo apicem versus evanescente instructa, denticulata. Foliola perigonialia intteriora obtusa in processum filiformem extensa. Anumbts simpliciter firbriatus. Hedu. $l$. $c$.

54. 11. decipiens, caule ramisque depres. sis pinuato erectinscnlis vagis, foliis im. bricalis lanceolato-acuminatis concavis enerviis, capsulis erectiusculis cylindraceis.

In arborum cortice, ligno emortuo. (Color sericei; folia in siecitate erecta, vel sulbsecmuda ut in cupressiformi; ad lentem integerrina ener. via longo cuspide terminata. Srruculi ranosi apicem versus vagi pinmati breviores sustim, ex quiluns setue Ureves glabrae, Capsulae cylindraceae demum obliquae. Operculum acutum conicium.)

55. H. plumosum, caule reptante, ramis brevibus pimato - erectiusculis incrassatis confertis, foliis imbricatis subulatis acuminatis, capsulis erectiusculis. L. Dicks. hort. sicc. brit. 5. 22. Dill. Musc. t. 55. fig. 16.

In saxis et arboribus. (Color splendens seri. cens cx luteo ruicscens. Folia per lentem in. tegerrima orato vel lasuceolato subutata in lour.
guin 
gum acumen jroducta nuinervia: nervo apicen jon allingente. Setae breves flabrax". Capsulae erectiusculac demum sthicelun.de. Var. niust: folis anguscioribus, ra:nis Lreviosibus, operculis conico - aculis.) Al live itague divcrom : lípum pluszosum, trumco reptaule ramisyue: primariis lifaluis druressis; fuliss longins acumi. matis, striatis; thecis e jedunculo laevi cero. ruis; operculo conico. Hedu. 7Iusc. 4. \%. 15. quod ol locum maturaleu non roale diccretur:

* H. salebrosum, caule reprante, ramis comfertis pimblo - depressis s foliis niatis acutis $\overline{3}$-strintis: stris oppositis injequa. libus; caprulis e seta laevi cernuis, opéculo conico.

Locis saxosis; Vere.

56. 1. polyanthes, caule repente ramosissimo multiliono, ranulis capsulisque erectis. Schreb. spicil. 1n70. Hedw. IVIusc. 4. t.2. (Lestica polyautha.)

Ad truncos arbonum autosarum, cumprimis salicum (Lips. Schreb. Hedw. 117 cgap. 7 imm.) Vere, Autumuo. (Folia ex ovato in longun cuspidem, pilum nudo oculo facile menticnten, protcusa, concava, vasorum la-ciculo destiula flediu. ?. c. lil sicco folia subsecumda, ranuli sacpe incurvi.)

* 57. Ir. sericram, caule repente, surculis cunfertis crectis, foliis Inuceolato acumiwatis striato-uervosis, capslilis crectis, scis tuberculatis. I. Dill. Miusc. t. 4=. $\sqrt{2} \mathrm{~g}, 59$. 
Curt. lond. Hedw. Musc. 4, t.17. (Leskea sericea.)

Ad arbonm truncos frequens; Vere. (Surculi juntores in siccitate curvati ( $A$ priori dif. fert : surculis crassioribus et lougioribus, foliia per lentem tristriatis actuioribus; setis exaspe. ratis.)

58. I. lutescens, canle surculisque vagis depressis exporrectis ; foliis ovato - lanceolatis acuminatis striatis, capsulis ce:muis, operculis oblique conicis, setis exasperatis. Schreb. spicil. 1071. Dill. Musc. t. 42. fig. 6o. Hedw. Miusc. 4. t. 16.

In pratis, nemoribus ad terram; Vere. Iongitudine et colore satis a prioribus diversinm. Theca nomunnquam basi apophyscos in modnus angustata. Folia tristriati Hediv. l. c. Hypunm ramusum Neck. Leers, herb. 88o. Leskea lon. gifolia Ehrl. crypt. cxs. 28 i.

59. H. piliferum, caule decumbente, ramis vage pinnatis, foliis ovatis concavis obtusis apice piliferis; operculis cuspida. is, setis exisperatis. Schreb. spicil. 1059 . Hedw. Mrusc. 4. t.14.

In nemorosls umbrosis udis hinc inde cum priori (a quo statim folits et operculis dignosci. itur.) Vere. (Var. ramis brevioribus fascicula. tis, operculis brevins cuspidatis; foliis ovato. lanceolatis in pilunn abeuntibus. v. Hedw. l.c. fig. 5.)

* 6o. r. striatum, caule decumbente, ramis yagis recurvis; foliis patentibus pli. F 
casn-striatis; opersulis mucronatis, setis glabris. Schreb. spicil. 1058. Hediv. Musc. 4. $t .13$.

Tn nennorosis udis; Vere. (Folia caulina se. mi - amplexicaulia, cordato - triangularia, ramea ovato cordata plus minusve acuta, in sic. citate praesertim striato - plicata, uninervia: dicrvo apicen nou atingente, narginc temissi. me fermlata; pallide viridia, aquelse pellucida: Hypum longirostrum Ehrh. cryph. cxsice. 7. Hypum lerevirostrum, caule decumbentc, ra.. mis ragis recurvis, foliis patentibus multi.stria. sis apice inflexis apiculatis serratis. $I / 2 r / h$. l. $c$. S5.)

61. Ir. strigosum, caule repente, sirculis ramisque abbreviatis erectis apprnximais ; foliis orato. lanceolatis imbricato - patulis; capsulis cermuis.

Ad terram: (Foiza ler lentcm ovato oblonga! - scrmata nervosa. Sctac ex caulc, glabrae pe. rigonialibus interioribus lanceolatis longe acu. . miuatis piliferis quasi foliis cinctae. Operculum ucuminatmm conicum. Ramuli atcumati, seta duplo breviores. Setis et folits, illis glabris istis haud vel parum acuminatis breviorihus, oper. culis acmminatis longioribus praccipue ob $\mathrm{H}$. pelutime dignoscitur. Hujus loci ctian videtur Hypu. intricatum Timm, megap. 1200, quoad "iccimina viva Aucioris.)

62. H. murale, caule repente, ramis compressiusculis ; foliis ovatis acutis; capsulis subcerntis, operculis tenuissime rostratis. Hedw. Musc. 4. 50. 
Ad muros, ad sylvas montosas; Vere. Folid ovata, ad lentem concava brevissime acuta te. nera, nervo teuni apicem non atimgente percursa, seta rnmulis vix longior, laevis. Oper. cultum in tenue et longum rostrum productum, varie curvum. Surculi in siccitate compressiusculi.)

63. H. ruscifolium, canle repente, surculis ramosis erectiusculis teretiusculis, foliis ovalibus concavis; capsulis subionndiscermuis, operculis rostratis. Neck. Meth. 181. Dicks. fasc. crypt.5.10. Dill. MLusc. 38. fig. 31 .

Ad saxa, in sylvis nmbrosis. (A priori dif. fert: surculis crassioribus, folits majoribus, ovi. libus brevissime acutis, nervo breviori insigui. tis pellucidis viridibus. Capsulac crassiores.)

*64. H. rutabulum, caule procumbente vario, surculis subramosis erectis curvatis, foliis lanceolato - acuminatis patulis; capsulis cernuis, operculo conico, setis muricato-exasperatis. I. Dill. Musc. t. 58. fig.: .9. Hedw. Musc. 4. t. 22.

Locis humidis; Vere. (Folia ovato - acuminata concava uervosa : nervo apicem non atrin-gente; apice tenuissime serrulata. Opcrculum conicum brevissime apicnlatum. Variat colore, setarum longiudine et ramorum crassitic.)

65. I1. riparium, caule prosirato varie diviso', sniculis radicantibus, folits ovatoacutis disichis; capsulis cernuis, opercu$\mathrm{I}=$ 
lo acuto', setis glabi is. L. Dill. Mirsc. t. $\{0$. Jig. 44. C. Hedw. Musc. 4. t. 5 .

In aquosis; arstatc. (Folia integerrima acu* minata adpressa bifariatn patula uervosa: nervo apicem versus evallescente; periguniatia multo breviora quam in priori et minus actuminata; Eetae breviores, capstilae graciliorcs.)

*66. 11. rivulare, caule surculisque elon. gatis, foliis ovatis acutis concavis; capsulis ovatis, cermiis, operculis acuminatis. Elirh. crype. exs. 252. Mediv. Masc. 4. 1. 4. (Hypmum riparioides.)

In aquosis, rivulis; aestate. (Folia obata, ovato-lanceolata plus minusve acuta, plexum. que atro virentia, per uargines ad lenten denviculata nervoque apicrm non attiugente instrte cta. Sctac slabrae breves; capsulac crassinscu. lac, operculum rostratum, incurvum, recurvun. Habeo exemplaria pedali longitudine inferius penitus muda, residnis foliortun-exaspe. rinta. Hujus inci: Hypul. riesciforme Wris, $7 \%$. 47ytt. 225. Hypul. protixum, strculis rarnosiy clougatis inferue subundis, capsulis ovatis cilia. tis, setis adscendentibus abbreviatis Dicks. frisc. crotit. 2. 13. Dill. Missc. 1.38. Jig. 32.

* 67. 1r. Muitans, foliis lanceolatis, alternis remotis L. Vaill. par. t.35. fig. 6.

In aquis stagnantibus (Nassov. - Goll. IFeis, Lips. sichret. Berol. Willd. -) alosque capsulis. (Var. foliis et canlibus uniuoribus ; canlibus pis. untis; foliis angustioribus quasi in pilum exte. nuatis, subrecurvis capillaribus Dill. MIiss. $c_{0}$ 83. fig.7. Weis. l. c.) 


\section{(MUSCr).}

68. 7r. fontinaloicles, caule longo ramoso; foliis lanceolatis patulis; capsulis lateralibus. Heiliw. Musc, 5, t. I/, (Tricho. stomun fontiualoides.)

In acquosis, arbonm ripariamm radicibus (Fificu Natlerer Klagenfurehi Wulfen. Fonti. ualis minor Jicks. cotfect. of dued 7, . I7. Scta perbrevis cum capsula foliis perigonialibus cin. cti. Perist. deutes 16 , apice in processus filiformes $z-4$ fissi, basi perfurati.)

\section{6. ғорітіNaxis (Hülimoos) G. pl. 3655 .}

Peristomium duplex: exteruis dentibus sedecim, imterins reticulatum. Capsulae seta brevis, perichatio imbricalo obvoluta.

* 1. antipyretica, foliis complicato-ca. ynatis trifarios acmis, capsulis Iatexalibus L. Dill. Musc. t. $33 . \sqrt{1 g} \cdot 1$.

In aquis, stagnantibus; Acstate, Autumno.

* 2. F. squamosa, foliis imbricatis subula. (6)- lanceolatis, capsulis lateralibus L. Dilt. Mrisc, เ. 53. fig.5. Hedw. MTusc. 3. t. 12. Dicks, liort. sič. 5. 25.

In rivulis montanis, subalpinis (Hercyu. Lom. meersclurf 2780 . I'ersoon.) Ang. Sept. (Folia atro. viridia, mudo oculo suloulata, ad lentem ener. via acuminata, laxe imbricata; perignnialia lata obtusa capsulam involvunt. Calyptra magna comici acuta, fusca, operculum lireve obmase coF micurs: 
nicum. - Omituo dubiam Fon. minorem, quae neque inter exsiccato. Muscos Lillenii amplius occurrit, ut pracscis corum possessor me edo. cuit, ill. Sibthor), neque eodem a Dillenus ine dicato loco practer rrichostomum toutinaloides sibele. Flor. oxon. 777. vicinior species reperta.)

\section{ANDREaEA (Andree) Ehrh. Beitr. x. 15 .}

Capsula serae perichactio imbricato ol)volutae, insidens, unilocularis 4 "valvis: medio deliscens.

* A. petrophila. EII Fr H. l. c. 1.92. (Iunfermannia alpina L.) Dill. Miusc. t. 73 .

Ad sixa (Hercyll. Mreb. Hassiac, Scharad.) In monte Spizberg bey Steelicu, Humboldt.) Antumio; Vere. (illos masculus terminalis aut lateralis, ex folis perigonialibus gradatim mino. ribus compo:itus. Folliculi spermatici f. anthe. rac oblongae, ovatae pallidac, Glamento brevi ris, ex foliis perignnialibus gradatim majoribus composims, genimlia complectens, calyptra ct wtylo iustricta. Capsula oblonga subecrigona, calyptrac parte inferiore abrupta cince, attollcus calyptram mimuam alluam pellucidam, api. ce ex persistente stylo nigram, acmam; smb gua rudimentum operculi. Scmina cxigua ro. ruda lutescentia.) 


\section{E CALYPTR T TA (Hepaticae.)}

28. IUNGERMANNA (Tungermannie)

G. pl. 1662 .

Capsula subglonosa unilocularis 4 - val. vis: apice dehiscens; sctae insidens.

\section{Frondibus cqulescentibus.}

* 3. I. excisa, frondibus caulescentibus, brevissimis teretibus subcompressis: foliolis et perianthiis arcte imbricatis, acute. emarginatis.

In umbrosis sylyaticis; Autumno. (Ex minuı. tissimis; confertin mascuntur can:lescentes frontdes depressate radiculosae, parum ramosac, vix man alteramve lineam longac, apice incrassazae, nundique foliolis : cmiausplexicanlibus disti-: chis, concavis, adpressis tectae. Capsulac cx. spice ramorum, brevi pechunculo instructae. Apices foliolornm ad lentem excisae ad antulum acut!um, fuscellae. Ilujus videtur loci: Inug. excisa, surculis repentibus ramosis apice Iloriferis, foliis iubricatis concavis ennarginatis, vaginis obovatis Dicks. fasc. crypte. 5. 11. t. "3. fig. 7.)

*2. I. cmarginata, frondibus callescentibus teretibus subcompressis: foliolis distichis imbricatis concavis parulis, obtuse emargimatis. Ehrh. Beitr. 3. 82. Crypt. excs. 21.7. 
In sylvosis montosis (Hercyn. Ehrh. Gït. Helder.) - (Color viridis, vel ex viridi plus mi. musve fuscus. Longitudo uncialis sescuncialls.)

* 3. I. julacea, Crondibus caulescentibus, revetibus subcompressis : foliolis arcte in... bricatis adpressis bifariis argenteis. L. Dilla. 7H usc. Lab. 73 . $\int 1 \mathrm{~g} .58$.

In montosis (Hercyn. Bruct. IFelw Ehrth. Has.. siac Schrad.) Alsquic cilpe. (Tolia ad lentem! obtuse emarginata. Frondes apicem versus in.. crassatac.)

* 4. I. setiformis, froudibus caulescentibus simplicibus teretibus filiformibus, erecris: foliolis partitis: lacinis lato-subufatis, ex.. terne comaliculatis, interue carinatis. Ehrh. Beitr. 5. 8ก. Crypt. exs. n. 17.

In Hercunia (F/2m/2.). Abrque capsulis. (Folia per lentem undique imbricata callaliculata simplicia adparent, accuratius inspecta nsyuc ad basiu quadripartita, margine hinc iude ciliata colore dilue ex fnsen vel viridi colorata. - ? Hujns loci : Inug. concinnala, surculis teretilns subcoupressis dense imbilcatis compactis, foliis convexo concavis glabris, foribus pedunculatis. Ligherf. scot. 786.$)$

Frondibus pinnatis: foliolis ciliatis.

* 5. I. trichophylla, frondibis teletiuscu. lis: foliolis capillaceis fasciculatis. I. Dill, Musc. t. 73. fig. 57. Schmid. Icon. 4. 42.

Locis montosis, nmbrosis; Antumuo; Vere, Foliola verticillatim vel alterne posita, ad len. em rubudata, ex bius frequentius teruis, rari. 
If quaternis ciliis, isthmis interceptis, couflata. Perianthia frequenter termiualia, apice lacinus: lata.)

*6. 1. Tomentella, frondibus deconupasi-

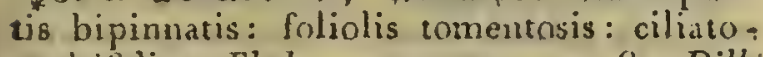
multifidis. Ehrh. crypt. exs. n. 8. Dill: Musc, t. 73. fig. 35.

Locis ndis umbrosis, sylvaricis; Abscue ca: psulis. Ihart. litl. ad ramonum divaricationes. (Ciliorum color ex unibus folia constant palli. dus, articula geniculati lougios:i quam in pritecedentilus. - lu1s: succulis pimuatis, repentibuts, supra tomentosis: foliis integris, tomenta. sissimis; stipulis mullis; amphigastrịis integris, tomentonis ; yeri-pluteriis cyliudraccis, , Jirsutis. filurh. Beir. a. 150. Hnjus locis Inug. ciliaris, TYeis. IT'3. Niecli. Hutuls. ctc.).

* 7. I. pulcherrius, rroudibus-surculisque pimmatis: foilolis stimulisque imbricatis apice 2-5- fidis, cillatis. TVeb. spicil. 214. Dill. Minsc. t. 69. fig. 5.

Ad truncos arborum, lucis humidis; absque capsulis: Ociolur. Web. (Frondes surculique crissiores lon fiores 2 - 5 ullciales, rami breves alterni isterruptc pimati, Golor ex viridi vel fusco ruliginosus, etiam laete virens. Folia la. tiora ciliis brevióribus, aefualibus iuter arıicı. Jormin spatia liand comractis, rcijculo augmstio. i stipata. Hujus loci: Iung. ciliaris L. Ehrh. ervyr. exs. "18. Imug. surculis pimatis, re: pcrubus, inpubibus; folits biradis, punctatis. ciliatis; scipulis unllis; amplizpastriis bilidis, $\mathrm{ri}$. liwtis; perisphacrito punctatis undis. Ejusd. Beitr. 2. 149.)

$$
\text { F } 5
$$


84 CNYPTOGAMIA ECALIXTATA

*. r. ciliaris, froudibus decompositis: fo. liolis duplici ordine imbricatis ciliatis; perianthiis termiualibus. Leers, herb. 907.

In Ligno semipurido; Aprili. (Var. frondi. bus angustioribus quan in specimine depicto: Folis mulo tenerioribus, ad lentem reticuli spatiis laxioriluns, ciliis longiotibus articulatis: articulis distiuctis oratis a seguenti facile di:stingnitur.)

Frondibus pinnatis: foliolis auriculatis.

*9. 1. albicans, frondibus pinuatis: foliolis amiculisque lineari-lanceolatis recur:vis distichis. J. Dill. MIusc. 1.71. fig. 20." t. 73. fig. 36 .

In nemorosis, udis umbrosis; Vere. (Frofi. des variant longitudine ab unciali ad sescumcia. lem. Foliola plus mimuve distantia vel approximata, superuc anriculata: animulis plernmque dinidio foliolo brevioribus; al lenten pice ar. guic denticulata. Perianthia cx apjec fiondis.)

10. 1. resupinata, frondibus pinnatis : fo. linlis imbricatis rotutudatis, superne auriculatis denticulatis. L. Dill. Musc. $t .71$. fig. 19.

In fissuris rupium; Verc. (Frondes recliuatae breves semunciales, parum ramosac. Folinla rotundata collcava, per amlinum rarius, verous auriculas frequentius deuticulata.) 
* 11. 1 . undulata, frondibus pinnatis: foliolis anriculisque integris. L. Dill. "Musc. c. 71. fig. 17.

In uliginosis montosis. Absque capsulis. (Fo. liola ut in pracedenti superue auriculata.)

* 12. r. nemorosa, frondibus pinnatis: foliolis auriculisque ciliatis. L. Dill. Musc. t. 7 1. fig. 18.

Iin nemorosis; Vere. 'Autumno in apice faliorum nigricautes antherae. Hedw. Theor. Musc. t. 15.)

* 15. 1. complanata, frondibus decompositis pinnatis: foliolis imbricatis complanatis rotundatis inferne auriculatis. L. Dill. Musc. 1.72. Sig. 26. Flor. dan. 1062.

Ad arborem cortices; Verc. (Frondibus la. uoribus, surculis et ramis aequalibus, pallide vireutibus, perianthiis compressis truncatis, fa. cile a priori diguoscitur.)

Frondibus pinnatis, inferne stipulis s. antphigastriis cectis.

*4. T. dilatata, frondibus decompositis pinnatis: foliolis imbricatis convexis rotundatis, subtus stipulis riplici ordine tectis. L. Dill. Musc. t.72. Jig.27. Ehrlo. crypt. exs. $n .2 \times 5$.

In arborum truucis, nupibus; Vere. (Frou. stes ut in prinri lineares, angustae, magis tamen linc inde apicem rersus dilatatae, colore plus 
Plus minusve ex viridi fusco, sicro. Stipulac laterales romundatac, incdiac sparsac ovato-oblongae remotae, apice lifidac.)

* 35. I. tanariscifolia, fromdibus decom. positis piumatis: foliolis imbricitis adpres. sis subrotundis, subus siipulis triplici or. dine iectis. L. Dill. I12use. t. $7^{2}$. fig. 51.. Schmidel ic. $t .67$.

In arberum trumcis, rupibus; Vere. (Fron. des colore phus minusre fusco, nitcntc. Sripu.. lac laterales oblongac parvae. indusversim aftixac; mediac slipiti aduatac margine revolutac, latiores, apjece cmarginatac, obọvatac. Foliólá perigonialia serrata.)

*16. I. supina, frondibus penuatis subpin-. matis, foliolis imbricatis bidematis, stipu-. lis in prona parte ovato - lancculatis imbricatis.

In umbrosis; absque capsulis. (Frondes bre.. re. linearcsimplices rel parum ramosae, super. ue depressac infernc convexae, stipulis mintis app tessis țculac; apice convo!

17. I. reprans, frondibus simpliciter pin:matis: folinlıs stipulisque subquadridcuta-

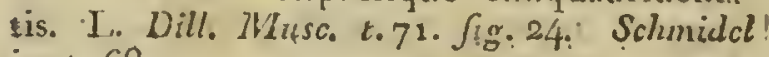
ic. $t .68$.

In umbrosis, ad vias, truncos putridos; All. tumno verc. (Ex tcuerrimis. Frons ranosa; sipes setis validus. Foliola ex ovato quadrata, apice profunde 24 . dentata : dentibus acutis. Stipulae simplici ordine remotar, $5: 4$ - dentatac. Perianliba ad basiu egredimum, allican. tị (ut tQta platata) apice tenuissime lacimnata.) 
18. 1. platyphylla, froudibus pinnatis: foliolis imbricatis adpressis cordatis; subi. sus stipulis triplici ordine tectis. L. Dill. Musc. i. 72. Fig. 32. (Lichenastruin arlois vitae facie.)

In sylvis ad truncos arborim, rupium; Vere. (Stipulue laterales oblongae, medias adnatae stipiti, apice reflexae, latiores.)

19. 1. radicans, frondibus pinnatis: fo. liolis imbricatis, apice.contractis 3 - denta. tis; subtus llagellatis simplici ordine sti. pularum tectis. ITich. gen, t.6. fig. 2 .

- Locis montosis sylvaticis; Abqque capsuliś. (Flagella filiformia longa undique ex stipulis minutis dentatis, in inferiore frondis pagina egredientia. Huijus loci:: Inug. trilobata iWeb. spin cil. 207. Ehr/2. crupte. exs. 12. 43.)

Frondibus pinnatis: foliolis inte- gris.

20. 1. pallescens, frondibus simpliter pirt riatis: "foliolis subrotundis integerrimis; stipulis lanceolatis apice bifidis. Ehr. crypt. exs. n. 302 .

In Hercyula (E/hrhart.) Absque caponlis.

21. I. asplenioiles, frondibus simplici. ter pinnatis: foliolis ovatis recurvis ciliatis. L. Dill. Musc. t.6g. fig. 5. Flor. dun. 2061 . 
In nemorosis udis; Trere. (Var. foliolis alter. nis minoribus Dill. $i$. c. Jig. 6.

*22. 1. viticulosa, frondibus simpliciter pinnatis: foliolis subrotundis intagerrimis: Dill. Musc. t.6g. $\int_{\mathrm{g}} \mathrm{g} \cdot \%$.

In ucmorosis udis; Vere. (Frondes breviores ex media latiori parte vel basi pedunculiferae. var. minor foliolis alternis ex snbrotundo linearibus.)

* 23. I. polyanthos, frondibus simpliciter pinnatis: foliolis subrotundis integerrinis oubimbricatis couvexis. I. Dill. Musc. t. 70. fig. 9 .

In umbrosis udis, sylvaticis; Vere. (Perian.. thia axillaria, apice per lentem lacimulata.)

* 24. I. sphagni, frondibus reptantibus sim-. pliciter pinnatis latere forileris, foliolis subrotundis integerrimis imbricasis secun-. dis Dicks. fasc. pl. crypt. 1. 6. tab. х. fin. 10. Vere.

Iu palustribus, Sphagno palustri adhaerens;

25. r. Lanceolata, frondibus simpliciter pinnatis lanceolatis apice capsuliferis, fo. linlis planis integerrimis obtusis. L Dill. Muse. t. 70. fig. 10.

Ad terram locis ndis, nmbrosis; Hieme, Vere. (Frondes quam prioris breviores, minus ramo- 
26. x. Gidentata, frondibus simpliciter pinnatis apice c: psuliferis, foliolis biden. tatis L. Dill. Musc. t.70. Jig. 11.

In sylvaticis, umbrosis; Autumno, Vere.

*27. I. bicuspidata, frondibus simpliciter pinnatis medio capsuliferis, foliolis imbricatis bidentatis. J. Dill. Musc. c. 7o. f. 13. Schrnidel ic. t. 63.

In montosis sylvaticis, nmbrosis; Vere. (Fo. lia quam praecedentis duplo minora, apice non raro in sterilibus globulifera. Hujus videtur lo. ci : Mnium Jissum Neck. meth. 277. fig. 1x.)

*28. 1. bicornis, frondibus simpliciter pin. natis, foliolis alternis distichis bidentatis globuliferis. Flor. dan. t.888. (Mn. fissum Lin.?)

In sylvaticis; Arstate. (Hujus loci : I. globu. iffera Tiznm, megap. 865. Frondes tiliformes wnlto longiores quam prioris.)

29. I. barbata, frondibus simpliciter pinnatis apice medioque capsuliferis, foliolis 3-4-dentatis. Schmid. ic. t. 48. Schreb. spicil. 1084 .

In umbrosis, sylvaticis; Vere. (Frequenter occurrit, nomine Iung. 4 : vel - 5. deutatae ven. ditata.)

30. 1. scalaris, frondibus simpliciter pinnatis: foliolis integerrimis ovatis, extremo canle distichis alternis, globulifero. Schmid. Iung. fig. 17. Dill. Musc. t. 51. fig.5. 
Iu nemorous, runestribus; Vere. (Perianlthium ad bisin caulis observo, neque vero foliola aurículata, dubitauter itaque adduco: I. Trichomenies, smiculis repentibus subtus auricn. latis, foliis ovito. subrotmudis subemarginasis, vagin oblonga-cylindracen lirsuta infera Dichs.

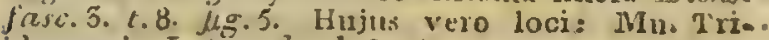
cllomanis Lecrs, herb. 8ะ7.)

* 31. I. pasilla, rroutibus hevissimis sim.. pliciter pinnatis: foliolis obtuse lobatis. Schmid. Ic- t.22. Mich. 2.5. fig.10. ist - In umbrosis, humidis; Acstatc, Arummió. (Periamhitum magnum campanulatum. Hijtits.o. ci : I. angulose, frondibus simpliciter, pintuins? foliolis latinsculis trumcatis obutse tridematis medio foriferis, theca campanulata Dicks. 'fotc." cropt. 1. 7. Lj. hort. sicc. brit. jesc. 1. 25.$)$.

\section{Frondibus acaulibus.}

j2. I. palmata, acaulis, frondibus digitato - jorlmaris, basi capsuliferis. Hedru. Theor. Inusc t. 13. fig. $9^{3} \cdot 9^{5}$.

Ad terram humidam, in cortice arbornm de. jecto, iu nemoros's (Nassov. Leers, her-b. Giz.

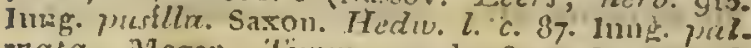
maia. Megap. 'Jinm, prodr. 879. Iu1g. pal. mula - Aumumo; Vere. Hujus loci vel pecu. liaris specis videmr: Inug. mnititida scthmit. 1.012. (.55.)

* 33. T. furcata, acanlis, frondibus linea. 1i. ramosis: extremitatibus furcatis oblusinsculis L. Dill. BIusc. t. 7f. fig. 45. Hediv. Theor. Musc. t. 19. 20. 
In sylvis ad trumcos arborum; Vere. (A se. quenti diguosciur: frondibus npice frequentet emarginatis subreflexis, sinul cun margine, ner. vo et perianthio ad lentem piloso-ciliatis, sub. tycllucitis: reticinli arcolis rotumdis. Hujus ct: iam loci: Iung. furcata Leers herb. gi2, ut ejus descriptio et specimina viva auctoris evin: cuint.)

*54. r. tomentosa, acaulis, frondibus ra. moso-furcatis lincaribus, obtusis, tomentosis.

Ad rupes; arborum truncos; absqine capśl. lis. (Hujus loci : Ira:1g. melsescens, Wchrank, primit." Tor sulisb. 860. Schrad. spicil. 76 . I. furcula Var. Teesdale's y. eborar. Trans. net. of Lin. soc.2.120. - Frondes elongatre; coloris in sicco prioris, pallidi; ad lentem opacac dénso tomento undique obductae, neque visibili reticulo instruciae.)

35. I. multifida, acaulis, frondibus bipimnatilidis L. Dill. t. 74 . $\sqrt{1} \mathrm{~g} .43$.

.. In pratis idis, sylvaticis, ad latera rivulo. mun, putridos tmucos; Vere. (Confertim nil. scitur; ex una basi frondes 4 -5. diltusae, in. ferius per mediam partem radiculosae, marpine undique liberac. Periamthia cylindrica apice ob. tusa angutiora, ad lentem snbhirsta, ex axul. lie pinnarmin prope basin adscendı:ut.)

* 36. 1. pinnizis, acaulis, frondibus oblongis sinuatis ex inferiore parte capsuliferis L. Dill. Musc. t:74. Jig.ja. Ivich. Gen, t. 4. fir. 2. Schrnicl. Icon. t. 55.

Tocis ndis, umbrosis, ad parietes rivulorum; Verc. (Variabili Indit magnitndinc ac forna, pro ratione aetatis, vel sexus. Perianthia cylin. drica obtuse cremulata. Color laete viridis, Gubstantia succulerita:) 
*37. I. epiphylla, acaulis, frondibus sinuatis medio capsuliferis. L. Dill. Musc. r.74. f. 41. Mich. Gen. t. 4. f. 1. Schmid. Iung. flg. 2. Hedw. Theor. Musc. t. $21-23$. i Locis udis sylvaticis, ad fossas et rivulos; Vere.

\section{9. marchantia (Marchantio) G. pl. 1661.}

Capsula subglobosa unilocularis apice de-": hiscens: dentibus plusquam 4; receptaculo communi pedunculato affixa.

*1. M. conica, receptaculo communi masculo sessili, femineo conico. L. Dill. Musc. t. 75. fog. 1. Schmid. ic. t. 3i. Hedw. Theor. t. 25. 26.

In nemorosis paludosis, nupestribus; Vere. (March. androgyna $W^{\top} e b$. spic. 230., quoad specimina eodem loco, in introitu speluncac Baumannianae collecta, frondibus nbstantia sencriore. areolis vix conspicuis, poris magis sparsis mino*. ribus, ceternm cjusdem magnimdinis quam in IM. conica.)

*2. M. hemisphaerica, receptaculo communi masculo peltato, femineo homisphaerico. Schmid. ic. t. 34 .

In rupestrihns umbrosis; Verc. (Hercyn., Pyrmont. Hannov. Ehrh. - Loculamentorim anguli 3 . 8.)

3. m. polymorpha, receptaculo communi masculo peltato, femineo radiato. I. Dill. Musc. t. 76. fig.6. t. 77. fig.7. Schmid. ic. t. g. 
t. g. f.2g. Bull. herb. t.291. Hedw. Theor. 6. 24.

Locis udis, ad scaturigines et fondes aquarum; Vere. (Cupulae gemmiferae s. viviparae, substantiae frondis insideutes margine deutatae.)

\section{бo. Throrona (Targionie) o. pl. 1663.}

Calyx bivalvis pericarpium mentiens, sub apice frondis.

r. hypophylla L. Mich. Gen. t.3. Schreb. Nat urforsch. 15: t. 5.

"Atii ripestribus. (Dresdae Schreb. - Exemplaria debeo cel. Smith, in Italia circa Neapolin collecra : A sketch of a Tour on the continent-Vol. 2. 73. - Frondes steriles ac fructificantcs, IVarchantiae similes, quuad viridem impellncidam smperficiem punctis albidioribus exinbetanibus adspersam, inferne nigricantes fibrillosa, arcte tegrae adglutinata. Fructifican. tes "compositáe obovatae, oblongae, apice obtu1. sae, rarins obcordatae, iuferins sub apice inunmesceures in :emirotumdum compressum bivalve veluti pericarpium, anterius promiunulum du. plici membrana structum : interiori laci objecta purpurascente. Frucus globosus albidus, ara. utcosis Glis crim pariete valvularum connexus, apice stylo fugaci coronatus subsessilis, demum major lutesceus irregulariter dehisceus, murge. scente uunc magis sic dicto calyce, ct, semini $\mathrm{i}$. formia demittens Iutescentia granilosa corpns. cula (quae immatura albida velnti semina mar. gine pellucido cincta emittere per microscopium mihi visa sunt) filis ex duplici helice membraua counexis,' tortis mobilibus aduexa.) 


\section{ANTHOCEHOS (Nadelscliorf) O. pl. 1664 .}

Capsula longissima subulata semibivalvis, calyce vaginati.

1. laevis, fronde plana sinuata L. Dill. Misc. 1.68. Fig. 2. Schanid. ic. t. 1g. Hedw. Theor. $6.2 \%$

rocis praminosis, udis sylvaticis; Attumnod (Capsularum et frondis magnitudine saturatior colore 2 sequenti statim distinguitur.)

* A. punctatus, fronde crispa laciniatad 1. Dill. Mlusc. t.68. Jig. 1. Selumid. jice t. 47.

In agris humidis, limosis; Alutumno. (Paraticula fibrosa calyptrac in ntraque specie capsue lae saepe adiaeret caduca.)

32. Beasta (Blasie) G. pl. 1665.

Capsula obliqua frondi inmersa, tubo extrorsum enronatz peristente.

* 1. H. pusilla, L. Mich. gen. t. \%. Dill. Mrusc. t. 31. Fig. 7. Sclmid. Blas. Hedio. Theor. t. 28 . vere.

Ad latera fossarum, rivulorum; Autumuo:

53. niccis (Riccie) G. pl. 1666.

Capsula subglobosa frondi inmersa tubo coronata trajecto.

x. R. minima, frondibus bi-tripartitis dichotomis linearibus canaliculatis acutis. 


\section{(HEPATICAE.)}

95

In terra limosa. (Superficics etiann per len. tem aequabilis, reticulo inconspicuo. Occurrit. apicibus obtusis, cordato-acutis.)

2. R. ciliata, Troudibus bipartitis, diclıotomis, margiue ciliatis. Mich. gen. t.5.7. fig. 5. Schmid. ic. 1.45 . flg. 4 .

- In terra limpsa, agris post messem; Antum. no. (Frondes angustiores, latiores, ad Jentẹm reticulato - punctitae.)

3. R. minoriformis, frondibus multipar. titis dichotomis acutis reticulato - caver. Hosis:

In terra limosa. (r. minori similis, frondes ad lentem laxe reticulatac.)

* 4. R. cazernosa, frondibus imbricatis ca= veruoso punctatis obtusis. Schmol. ic. t, 45. Fig. 5. (Ficcia crystallina.)

In terra limosa, ad piscinas. Iul, - Autum. no. (frondes orbiculares dilute ex luteo viren. (tes, )

5. 13. bifurca, frondibus multipartitis dichotomis apice bifurcatis, sulcatis. Schmid. io. 5. 4. fo s. 1. (Riccia glauca.') Mich. gen. $2.57 \cdot$ fig. 4 .

In terra limosa, humida. (Ex maximis, nudique saepe capaulis et gramulis tecta, diffusa, apice bifurca vel excisa.)

6. R. glauca, frondibus planis dilatatis bipartitis dichotomis obtusis. Hedw. Theor. t.29. Ehrh. crypt. exs. 13.115. (Riccia glauca.)

In terra limosa, agris post messem; Autum. no. (Color is recents ex viridi glaucus; super? 
ficies ad lentem acquabilis reticulato - punctata. Occurrit froudibus minoribus schmid. ic. $t .44$. fig. 2. Hiccia minima segmentis brevioribus etc.))

7. R. pyramidata, frondibus indivisis ob... longris, apice inferne triangulariter incras-. satis, fructibus pyramidatis. If illd. ins Bot: Mag. 4. 9. Mich. gen. t.57. fig. 2.

In rupe tenni tcrra tecta. (Hal: saxou. ad! Girbichenstein Willd. Lisze : etiamsi postca sol.. licite quasita, nou amplius reperta Bloctner)

* 8. R. fluitans, frondibus planis dichoro-ne multifidis divaricatis, furcatis apice: obtusis, reticulato-venosis.

Iu fossis pisoinis. (Ad terram humidam frondes hreviores latiores saepe conformes $f .2 . t .190 k$ Voill. paris.; aquae innatantes longissimae filiformes; pellucidae. Corpuscula littea sparsa ni. dulantur in tenera snlistantia, alia subtus con. epicrua seminifera globosa, superne iu porum:

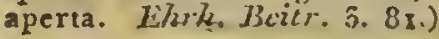

9. R. canaliculata, frondibus camaliculatis lineari-multifidis, dichotomis opacis.

Ad terram humidam, in fossis. (Per superfi. ciem frondes angustae, hinc inde ad leutem reticulato - cavernosa impellucidae.)

* 10. K. natans, fronde olucordata plana. subtus longis comptessis radiculis tectr $\mathrm{L}$. Dill. Musc. t. 78. fig. 18 . Schmid. ic. 1.74.

Inter Lemnas, in aquis stagnautibus. (Gott. Web. Palat. Neck. Poll. Barbi S'choll. -) Vere.

11. R. pellucida, fronte simplici $m \mathrm{~cm}$ bratracea tenera lobata: lobis obtusis obcor. datis: 


\section{(HEYATICAE.)}

datis. Mich. gen. t.57. fig.3. Dill. 4.78. fig. 12.

Ad terram humidam nmbrosam, in nemoro. sis (Nassor. - Ceers; Riccia crystallina herb. 252. Frondes $a b$ initio bilobac, sensim 5.5 . lobac et ultra, ad lentem reticulo venoso om. nium maximo, areolis pellucidissimis, margine hinc inde denticulis, cenero inferne radiculis instructac.) Autumuo; Vere.

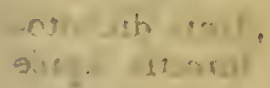

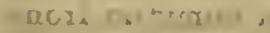

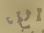

- ilit 3!. 


\section{S G UTELI. A T A (Lichenes.)}

34. cozrema (Gallerthechte) G. pl. 1668.

Irondes diaphanae, gelatinosac. Scutel-. lae nuarginatae.

* 1. c. Vespertilio, orbiculatum lobatum।

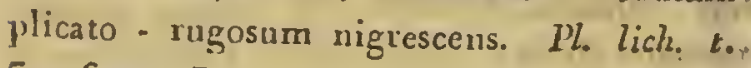
57. fig. $2 \cdot 3$.

In saxis, arborum truncis.

2. c. prasinum, gelatinoso - carnosum lo. latnm: lohis obusis simatis subimbrica. tis, tuberculis concoloribus; scutcllis ru.. fescentibus.

In rupibus.ad terram. (Nassov. Leers, Jerb. . 948. Lich. pulcher. Color in humido eleganter prasinus (lanchgrün). Frondes orbicnlares, vel l tuberculiferae, vel scupelliferac, in distinctis individuis; scuellarum wargo tunidulus parum crcuulatus.)

*5. c. auriculatmm, imbricatum sinuosólobatum crenulatum; scutellis depressis muricatis rufescentibus.

In saxis, muscosis. (Fercyn. - Mont. pinif. variicque Germ. locis. Ad radices arbor. A Coll. Tespert. difert: smbstautia crassiori lobis 
confertis margine crectiusculis, unedio depressis granulosis; colore in Immilo ex glauco vires. cente, niıricante, in sicco sordide virescente ex glauco et luteo mixto. Gramula superficiei demum in folia explicantur. Scutrllae infrequen: tes, margine saepe cremulato vel foliaceo cin: ctac, disco mifeacente. H. v. l. Licls. cochlea. tus, foliacens inembranaceus lobatus plicatus obscure virens, scutellis rufis Dicks. fasc: $x$. เ. 2. $(5.9 .9)$

* 4. c. tomentosum, subimbricatum sinua: titur lobatam, granulis superne villis in. ferine óbsituri; scutellis planis rufescentibus.--19 घม

In saxis, rupibus Arborum truncis; (Rarissime cum scritellis, margine foliis concolore ciu. oti5:a Colore obscure plumbeo, substautia semi. diaphana a priori statim dignosciur. Occurrit froude latiore rotunde lobata h. l. Lich. satur. uinus, foliaceus rotunde lobatus supra uigricans subtus villosus cinereus, scutellis rufis marginatis Dicks. fasc. 2. t. 6. fig. 3. Lich. myochurous Ehrte. crypt exs. 286.

* 5. c. granulosum, imbricato - aggrega: tum, crenulato-sinuatum gyrosum plicatum grauulosum. Dill. Musc. tab. 19. fig. 24 . 
In rupibus ad terram, absque scutellis. (Co... lor nigresceus. Magnitudo meialis sescuncialis.)

* 6. c. crispatum, imbricato - aggregatım । undulatum crispum, laciuiatum; scutellis terminalibus.

In rupilus, saxis. (Orbiculatim expanstum. A sequenti differt: fronde crispa undique, laci.. niis erectis latioribus, peripheriam versus cx-. pausis, margine graunlosis in plicis c̈um scutel-. lis terminalibus. - Occurrit scutullig rufis unargi-. ze granulatis.)

*7. c. Laciniatum, imbricatum laciniatum: laciniis multifidis lineari - caualiculatis cre.. mulatis; scutellis marginalibus. Dill. Muse. t. 10. $f_{\mathrm{g} .} 25$.

In rupibus, "saxis. (Var. magis crispa angtt. etior.)

* 8. c. glaucoscers, subimbricatum aggregatum crispo - lobñtum obtusum, siccitate cinereo-glaucum.

Ad aggeres, muros. (In prima aetate froudes parvae ex umbilicali radice lobato - divisae, ir. rigatae nigrescentes. Scutellac amplae fnsco sauguincac. Substautia pulposo-gelatinosa semidiaphana. II. vid. 1. Lich. cristatus IFulfen it Iacq. coll. 5. t. 22. fig. 2.) 


\section{(IICIENES.)}

*9. c. crispum, subimbricattum lobatum : lobis abbreviatis; depressum erectiusculum rotundatum crassiusculum; scutellis plaño-convexis marginatis. Dill. Musc.t. 19 . fig. 23.

Ad muros, saxa, terram. (c. crenulatum, sulimbricalum depressum sparsum, lobis abbre. viatis ; scutellis crenulatis.)

-192 6.1 $2315=0$. Coblit * 10. c. obscurum, depressum foliis oblitteratis; scutellis rufescentibus depressis obscure marginatis crenulatis.

-3r. Ad terram, nigrescens, arctissime terrae con. nzatum; frequeutcr iu consortio Br. stcllati.

11. c. crisiatum, imbricato-aggregatumn laciniatum margine cristatum.

In mpestribus. (Color ex prasino vel fusco vircscens. Thargo foliorum iucrassatus dentato. cristatus. H. vid. 1. Dill. IMusc. t. 29. fig. 26. $A$. $B$. fronde altius divisa et subdivisa canalicu. lata; c. fronde brevissime laciniata undique scn. tellis tecta.)

* ${ }^{2}$. c. marginale, subimbricatum lobatum: lobis crectiusculis sinunto - crenulatis concaviy; soutellis crenulatis. 
Ad muros, saxa, terram. (Gott. BernhardiFoliola lnci obversa diaplana viridia, margine. saturatiore cincta, semitremcllosa.)

13. c. polycarpon, subimbricatum aggregatum lobatum rugosum: rugis elevatis scutelliferis.

In rupibus ad terram. (Scutellae numerosissi. mae mediocris magnitudinis, ex rugis clevatis quasi pedunculatac, terminales, discum frondis penitus obtergentes.)

* 14. c. conglomeratum, Iaciniatum ereciusculım; scutellis,fasciculatis fronde majoribus. Eltrh. crypt. exs. 256 . (Lich, fa. scicularis.) Dill. Musc. t.19. fir. 77 . B.

In lignis mutridis, cortice, saxis., (H. v. 1 . Lich. Eascicularis Huds.)

* 15. c. fasciculare, aggregatum erectum Eyroso-crispun ; scutellis minutis obverse conicis stipatissime coronatum. Iacq. coll. 3. t. 12. fig. 2. (Lich. fascicularis M ulf.)

In saxis, inter $\mathbf{M}$ Tuscos. (Gott. Berrhardi Colore praccipue differt: c. argillosum, aggre: gatum gyroso - crispum pulposum sordide lutes: cens scutellis concoloribus stipatissime marginalibtrs et disci coronatum. Ibid.) 
* 16. c. Botrytis, aggregatum crispura substipitatıin polycephalum.

Inter Muscos cum sequenti. Color niger. Scintellae minntae obovatae convexae, apice hivic inde depressae, in capitula quasi granulo. sa substipitata connatae.)

* 17: c. pannosum, agrregatum, multifidociliatum.

Inter Inuscos (panuo nigro scriceo ad mudos oculos simile pulvinamm; Per lentem nsque ad basiu mullipartitum: lacinii erectis acutis linea. Tibus cơnferrissimis.)

"s 2 8. "c. higrim, byssinum nigrum; scutellis immersis concoloribus. Enum. Lich. t.3. $\sqrt{ }$ g. 6. " (I ich. niger.)

In rupibus, saxis. (Cavendum ne cum ste. reocant. corallinoid. vel Byss. velut. confundatur. Per lentem foliolis. subpellncidis tremello. sis lin eariłns subdivisis, aquam citissime bibulis compositum; scutellis immersis.)

19. c. tenuissimum, agggreģatum fuscovirescens, digitato multifidum; scutellis subimmersis fusco - rufescentibus margimaris. Dicks.fasc. 1. 5.2. fi.. 8. (Iich, tenuigsimus,) 
IOf CRIPTOCAMIA SCTTELIATA

Ad terram aggeres sabulosos. (Laciuiac ad lentem acutae. Colore, folionm divisura et: scutellis acque a pamoso ac scquenti diversum.)

*20..c. pulvinatum, aggregatum erectmm laciniatum truncatum creulatun. Dill. Musc. t. 19. fig. 5 f.

Tuter IMuscos, ad muros. (Absque scutéllis. Substantia semigelatinosa. Frondes confertissie? mac pulvinatae nigrae.)

*21. c. ciliatum, erectum aggregatuin hr membranaceum lividum, laciniatum planiusculum, margine ciliatum. Dill. Musc." t. 19. fig. 31. A. B. Leers, herb.947. (Lich." tremelloides.)

Ad terram inter IVIuscos. (Scutcllae Tarissi-" mae, uninutae froudi insidentes eaqué pallidid? res, hinc inde ciliatac, disco carueo concaro, Var. maguindine et colore plus minusve in plum. beum vel cacmleum vergente, froude sacpe plane. lluda. c. fimbriatrm, erectum aggregatum, foliis membranaccis laciulatis, margiue dense funbriatis. Dill. l. c. C. Absque scutel. lis. lisdem cum priori locis, quo differt: co. Iore Inagis fusco, ciliis densis, ad lentem ra. mosis.) 
22. c. corniculatum, laciniatum menbranaccum convolutum multifidum corniculatum. Dill. Musc. t.1g. fig. 3o?

Ad terram. (Color in sicco lividus hine ìn. de subfuscus. Substantia tenuis non multura gelatinosa. Frooder cucullatac.)

23. c. lobatum, depressum sulimbrica. tum margine crenato - lobatum: lobis rotundis; scutellis depressis concavis fulvis margine fronde concoloribus.

- In terra, IMuscis. (Color e glance viridir, substantia crassiuscula. Colore occurrit nigrescente, livido.)

*24. c. subtile, depressum, foliolis minutis angustis divisis: laciniis inaequalibus S6hrad. spic, 95. (Lich. subtilis.)

Ad terram limosam in viis cavis sylvaticis. (Gott. Schrad. -)

"25. c. byssinum, depressun, foliolis minutis granulosis scutellisque demum convexis.

Ad terram limosam. (Fóliola sudo oculo gramlis minitis'simillima; ad lentem inarqualiter et brevissiose laciniata.)

$$
\text { * } 26 \text {. }
$$


26. c. graniforme, depressum foliolis graniformibus; scutellis margine crentilatis.

Ad terram limosarn. (Foliola mado ocnlo graa niformia, ad leutem line inle inciso-crenata; scutcllae quam praccedentis duplo majores, ing tegrae vel cremulatae, dịsco depresso.)

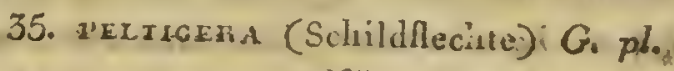
1668.

Frondes coriacere. Scutellat compressate margine adnatae.

" 1. P. canina, plana obtuse lobata subtus velloso - villosa. Iacq. coll. 4. t. 14. fig.1. (I.ich. cauinus INulf.)

In sylvis ad terram. (Occurrit paucioribus' subrotund is scutellis, fronde latisima : lobis ob. tusis rotundatis einc verrucis glabra: P. anf. plissimer, aphthosae simillima.)

.2. P. polydactyla, adscendens polydaçty. 1a, subutus velloso - villosa. Pl. lich. t. 4. fig. 1. Incq. coll. 4. t. 14 fig.2. (Lich. polydactylis $N^{\prime} u l f$. )

lin sylvis ad terram. (Var. minor tenerior pellucida. Dill. Musc. t. 28. fig. 108. Scutellaec guam praecedentis duplo minores.)

5. $\mathbf{x}$ 


\section{(CIOHENES.)}

5. p. rufescens, plana lacininto lobatn: lobis siunato - crispis, subtus venoso - villesa. Iacq. coll. 4. t. 15 . (Lich. rufescens Ifulf.): Dill. Musc. 1.27. fig. 103. .

: Ií sylvaticis, rupestribus, ad terram. (Scu. rellac subrotundac, demum adscéndentes cum foliorym lobis.)

* 4. 1. horizontalis, plana laciniáto-loba. ta; scutellis horizontalibus planis. Jacq. coll. 4.1.16. (Lich. horizontalis Wulf.) Dill." Miusc. t. 28. fig. 10\%.

: In sylvaticis', nupestribus. (Subuus margine aveñia nigricans reticnlata. Individua alia margine pulverulęrta. . Var.: polydactyla scutel. lis minoribus.)

$\because$ 5. 1.: aphthosa, plana diffusa obtuse Io. bàta, superne verrucis nigris, inferne.venis concoloribù fibrillisque longissimis tecta. Ml. lich: t.6. Jis. 1. Jacq." coll. 4t. t.17. (Lich. aplithosus Tiulf.)

In sylvaticis mpestribus, ad terram.

*6. venosa, adscendens, subtus venosa; 'scutellis horizontalibus planis orbicu' latis. Pl. lich. t.6. fig.2. 
Locis nmbrosis elatis, at margines viarum. (Totily gentris minima, fronde ovata, obovata; Rabelliformi.)

* 7. papjracer, subcoriacea, laciniata obtuse lobata utrinque glabra; scutellis posticis.

In abietum cortice, ramulis dejectis. (Ab muciali usque ad semipalmarem latiudinem. Scu. tellac rufo.férugincac, demnum amplissimae , a fronde marginatae. Lisiam, ad lentem maxime allgenteun subius vix comentosa; diversa itaqùe videtur : )

8. P. tomentosa, subcoriacea;-- Iaciniata lobata utrinque toniontosa; scutellis pó.. sticis.

In montosis rupestribus ad terram. (Substantia tellaciori, tomento ad lentem / superue, in convexa potissimum scutclarun parte, inferue mudis oculis facile obscrvabili, dew6o, dilute fusco, linc inde punctis albidis, excavatis, quod ctiam in exemplaribus in Svecia lectis co. ram vidco, iulerspersis, F. sequentis in modum, saltem ut subspecics distinguatur a praccedenti. Sÿlonyma Dill. MTuse. r. 28. fig.5. Iacq. coll. 4. c. 12. Jig. 1. Xich. resupinatus, IFulf. Web. spicil. 267. hauc val illaw iudicant. uncr papy- 
raceam non raro occurrunt individus ascutellata, margine glauco pulvere adspersa, crispa.)

*9. P. sylvatica, subcoriacea, sinuato-la. ciniata scabia, subtus tomentoso-villosa punctis excaratis albidis conspersa. $P l$. lich. t. 4. fig. 2. Iacq. coll. 4. t, 12. fig. 2: (Lich. sylvaticus $\nu V_{u l f .)}$

Locis ylvaticis rupestribus. (Scurellae infre. quentes, parvac, in extimis laborum laciniis. adscendcntes, quibus praeter odorem gravem in humido, distinguitur a consimili plainta; Loba. ria fuliginosa: Lich. fuliginosics, foliacens re: pens sinuato - lobatus scaber, subtus. spontioso; villosus lacunosus, scutellis planis ferrugineis, marginibus pallidis. Dicks. fasc. 1. 13. Dill, Musc. t. 2g. fig. 1oo.)

56. UMBILIcaria (Nabelliechte) G: $p l$. 1668.

Frondes foliaceae peltatae. Scutellae contortae.

* 1. v. polyplyylla, imbricata ntrinque glabra, superne fusco - senea, inferne atra.

Iı montosis Iupestribus. (Fercyn. - raro scutcllifera' irrigata fusco - vircus. Euara fron. de sirplici occurrit, h. 1: Liclı. glabes Affar. $+1$

II 2

iR 
in kongt. Fet. Acrd. IIandl. 15. 1.2. Jig.5. utrinque atra $\mathrm{h}, 1:$ ).

* U. antlracina, subimbricata glabra, superne et inferne atra. Iácq. Misc. 2. 1.9: fig. 4. (Liclı. anturacinus $V I$ ulf.)

In saxis montosis. (Hcrcyu. - frondçs obu. se lobatac rigidiusculae, irrigata vix colorc diversae. Specimina e Carinthia a rev. Wrulfer missa, tcmiora, margine crispato - lobata.)

2. v. flocculosa, undique muda sublae, vis, fusco - nigra, inferme sparsim lacuvo: sa. Iacq. coll. 5. t. 1. fig. 1. (Lich. Alocculosus TTulf.)

In saxis montosis. (Exemplaria in Carin th, lecta rigidinscula farina adspersa uigricante, per len. tern in papillas conicas clevata, lacunac subtus parym impressae - Dpsaliae lecta tenuiora, pulverc etiam per lentcm similari, conspersa, jnferne distucte lacunosac. Scutcllac rarissimac depressac contortac. H. vid. 1: Lich. deusus, sunbil. intcger sublaevis ater, subus impresso. punctatus rugulosus, peltis depressis H'estr. in Fongl. Vet. Acad. Handll. 14. 4j.)

* 5. ช. hyberborea, fusco-nigvicans utriuque nuda, superne rugoso-papulosa, sub- 
tus laevis olisolete lacunosa. Achar. l: $c$. i. E.: fis. 2 .

In montosis rupeseribuss. '(IIercyn.: - Froifdibus etian inbricato - lobatis.)

* 4. u. srisea, subcincerea laeviuscrila, sub. tus nigro - fusca papillosa. Achar, l. c. t. ?. fis. $3:$ :

In montosis rupestribus (Hercyn. - . . . . L L $f_{2}$ * 5. v. erósa, nigricáns, inciso lobatay

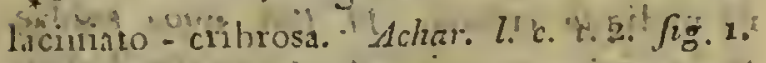
Ehth."crypt. exs. 506 . (Lich. corrostis.)

In noutosís rupestribus (Hercyn. - II. 1. Lich:' Cribcllum Retz. scrid. ed. 2. 26r3. Tnferme diliztior, hiicindé reticulato : fibrillosa. Occurs. iit ctiam parum erosa luci obversa tamen crí: brósá.)

* 6. v. pustulata, snperne pustulata infla. ta furfuracea, subtus lacunosa. Pl. lich. t. 28. fig. 1. 2. t. 2g. fig. 4 .

In montosis, saxosis frequens. (Scutellas di. sincte et spiraliter contortas observo in exem. plari et Arerica septentr. a D. MTululenbers accepto. Hercyn. rarius scutellifera margine simplici, disco depresso.) 
* 7. U. corrigata, undique nuda, superno reticulato-Jugosa. Pl. lich. t. 43. fig.4-7. Ehrh. crypt. exs. 8g. (Licl. mesenteriformis.)

In montosis rupestribus. (Hercyn. - Fibril. lac hiuc inde sparsae. Substania temuiori, $\mathbf{m}$. gis plus minnsve elevatis conccutricis a sequichit diversa. II. vid. I. Lich. pullus Wrulf. in: Iacq. Misc. 2. 6.9. $/ \mathrm{gg} .3$.

*8. v: crinita, imbricato - plicata margine undique crinita. $P l$. lich. t. 44. f. $1-9$. Hedw. stirp. 2. t. 1. A. (Licli, proboscideus.)

In montosis, rupestribus frequens, (Actate frondes corrosae, nigosac, hiuc inde ta!n!lum erinitae. Color e cinerco nigricans, subuns di. Intior fuscus. v. rigidie, imbricato - plicata undique mda. Cum priori. IX. I. Tich. mescuncri. formis Wulf. in Iacr. MTisc. 2. i. 9. fig. 5.)

9. ช. hirsuta, cinerca làcviuscula, lobàta: lobis inflexis; subtus lirsulissima fibrillis simpliciusculis. Achar. l. c. t. 5 . fig. I.

In montosis. (Supcrne cinerea, per Jentein utrinque grautulata, subtus fusco - cincrea, rädicen versus nigricans. Fibrillac cincreac sim.

plices, 


\section{(MCHESES.)}

plices, hiuc inde furcatac. In excmplaribus Upsaliae lectis, 1.4 incialibus, nigra scutelli. formain impressa puncta observo. Praeter mino. rem uncialen magnitudinem et rigidiorem in sicco texturam parum differt:)

* U. spadochroa, cineréo-glauca, laevin-. scula, sibutus sparsim fibrillosa papillosa subfusca. Ehrh. crypt. exs. 5r7:" (Lich. spadochrous.)

[11 montosis. (Hercyn. - H. 1. quoad specinina a cel Smich ex herbario linueano me. cum communicata : Liclıen polyruhizos Lin., in. ferne cincreus subfuscus vel fitsco-nigricaus, șcntellis distinctis contortis. Irrigata parum virescit frous, ctiain polyphylla. Alicua et cum r. cirrhosa jill. lich. t. 2. fig. 3. potius conveniens species est: Lich. polyrrhizos Achar. l. c.t.2. fig. 4.)

* 10. v. velloa, fusco aenea - glabra lobata, subtus margineque hirsutissima atra. Pl. Lich. t. 26 . fig.5. Achar. l. c. t.3. f.2. (Lich. pellitus.)

In saxis, rnpibus montasis. (Hercyn. - Var. fronde minori polyphylla, subtus atra centrum versus unda, papillis exasperata. - H. 1. Lich. hirsutus Suartz. Westr. l. c. 47. Magnitudine palmari aeque ac fibrillarum structura differt: H 4 Lich. 
Lich. velleus quormudam Dill. MTusc. t. 82. fig.5, ut specimina lecta eodem a Dillenio indicato loco, e Pensylvania a D. Muhlenberg accepta, cvincuut.)

\section{7. cradowra (Strunkfleclite) O. $p l .16 \dot{6} 8$.}

Frondes caulescentes teretes intus carae. Scutellae tuberculosac.

\section{Fruticulosae.}

* 1. c. rangiferina, perforata ramosissima xamulis fastigiatis nttantibus. Dill. Musc. . t. $16 . f i g .30$.

In exicetis, montosis; sylvaticis: (C. syluati. $\mathrm{ca}$, longioribus saepc palmaribus canlibus ere-

'clis ramoso - fastiriatis nutantibus, scutellis ftr. scis minutis.)

*2. co raccmosa, perforata samosa: ramu- lis scutelliferis racemosis erectis.

In cricetis sylvaticis denso cespite. (Caules undinsculi hinc inde foliolis adspersi, superne parum ramosi, ultimo racemosi, undique scu. tcllis rufesccutibus obtecti.)

* 3. c. rangiformis, perforata ramosissima: ramulis malis brevissimis furcatis ereetis fastigiatis. 
Iu cricetis, montosis. ( (Apíces ramulorum fu. scac. Scutellae sparsac. Caules tenuiores humi. liores magis divaricati. quam in C. rangiferiua. Fig. 9. Ser.3. Sect. 15. t.7. Moris. habilum ion'male exprimit.)

* 4. c. furcata; perforata ramoso-divaricata: ramulis furcatis nudis scutelliferis rudiatis. Dill. Musc. t. 16. fig. 27.

In ericetis sylvaticis. (H. I. Lich. furcarus Iiezch: francof. 859 , quoad specimina viva Auctorifir Semifusca in'sicritate. Caulibus-1ongio. ribis ramnlisque subulatis Moris. hist. Sect.xy.

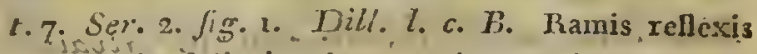
atteunatis foliosis glanco virescentibus , $\mathrm{C}$. re. curva. Dill. l. c. D. Yrill, bot. t.7. fig.7. Cis ifurcato-subulatre, perforata simpliciuscula erectr superme ranosa furcato - subulata, ultimis ramulis brevissimis. Vaill. paris. $t .26$. fig. 7 .

* 5. c. spinosa, perforata ramosa diffisa: rumis divaricatis : ramulis digitato - multifidis scutelliferis. Dill. Musc. t. 16. fig. 25.

In ericetis montosis, sylvaticis. (Cinereo : vi. rescens, foliolis adspersa, supcrue ex cinereo Hinc inde subfusca. c. spinescens, ramis trifi. dis: ramulis sterilibus, brevissimis fastigiatis.) 
*6. c. ceranoidcs, perforata diffusa ranio'sa : ramis divaricatis nudis superne incras6ntis: ramulis fastigiatis truncatis.

In exicetis moutosis. (Hercyn. - Color $8 \mathrm{~m}$ perne ca ciucreo subfuscus; magnitudiue uncia. li sescunciali. H. 1. Lich. cercuzoidęs, fromde tereti aculeata, alis perforata laevi veutricosa : ramulis terminalibus subradiatis Neck. meth. 63, quoad viva" exemplaria Auctoris. "Axillt ramulo." rum hiuc inde perforati)

* 7. c. uncinata, axillis perforata radiatum mcinata, iucana, inaequaliter ranulosa. Dill. 'Musc. t: 16. fig.21.' A.

In ericctis montosis. (Hercyil. - Pnlverü jentä incana superficic, ramulorum dirisione? magnimdine bi - trimeiali, facile a sequenti dignosciur.)

* 8. c. Linncialis, axillis perforata spiuu. losalsimpliciuscula nuda; ramulis brevissimis fastigiatis acutis. Dill. Musc. i. 16. fig. 21. H.

In ericetis montosis. (Parnm ramosa : ramulis : sterilihus brevissimis subulatis $2 \cdot 3$, stcllatis, cil-. spidatis apice subfuscis, rarins scutellis pallide: carucis yel supfuscis terminatis, in figura 2. Mich. 1. 40. beste expressis. Miorisoniana C. 7. fig. 7. 
fig. 7. Sect. 15, habitum nostrae stirpis melius quam Dilleniana repraesentat. Color pallide ex iutco alvidus.)

*9. c. uncialis, perforata nuda brevitey ramosa et ramulosa: ramulis acutis. Dill. Masc. t.16. fig. 22.

In cricetis: (Ex humilioribus; yel incrassata yel aiteiuata apicem verstus; in quibusdam superficiem tuberculasam, val papulosam potius olservo.)

Bruto. Papillaria, simpliciuscula ventricosa, obtusa, apice subfusca, adscendens xamulosa scutellifera, Iacq. coll. 3. t. 3. fug. 2. (Lich madraeporiformis Wulf.) , "Ad terram in ericetis, montosis. (H. 1. Lich. Prepillaria, fruticulosus, fistulosus, aphyllus, albitins; ramis pancissimis, obtusis, brevissimis tuberculis terminalibus, carneis Ehrh. Beitr. a. 105. Crusta albida.)

t

c. molariformis, adscendens brevissime yamulosa:-ramulis confuentibus truncato fastigiatis apice fuseo-nigricantibus. Dill. Musc. t. 16. fig. 28.

lisdem cum priori locis.

Subu. 


\section{Subulatas.}

*11. c. sulalaira, perfr zata simpliciuscu. la crecta, ramis simplicilus subulatis. Dill. Muse. t. 16. ftw. 26 :

- In cricclis, sumosis. (Glabra, cinerca, vires scens, scmifusca; ramulis liuc inde dicliotomio Iamosis.)

12. c. subuliformis, adscendens filiformi-

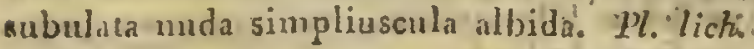
t. 29. fig. 1 - 5 .

In montosis inter manscos. (Hercyn. $-E /$ trti. Jich. subuliformis, fruticulosus tubrilosus, fili: formi - subulatis, simplex, cespiomo adsceń. dens, lacvis, afbissimus", ramulis paucissimis: urevissimis, sacpe nullis Beilr, 3. 82. Lich, ver. suicularis Lin. Jil. musc. 37.)

- 13. c. taurica, àdscendens cespitosa ven.. iricoso - subulata, subramosa nuda virescentii. albida. Pi. lich. t.5 4 . fig. 2.

In montosis. (Carinth. IVulf. Tyrol. -)

14. c. furcellata, simpliciuscula incana, simperne dichotoma furcata; ramis subula.. tis. $\operatorname{llag}_{\mathrm{g}}$. lich. 1.2. fig. 10.

Iu ericeris, sylvatiois. 


\section{(IICHeNss.)}

\section{Subulato - scypliferae.}

- 15. cr comata, fronde foliacea stipite in cano obscure scyphlufero, ventricoso súbulato. P\%. lich. t. 25. fig. 1.

Int ericetis, putresccute ligno. (Var. Iongis cylindricis, stipitibus simplicibus, brevioribus ventricosis, subramosis, foliolosis, cocciucis vel Eubfuscis scutcllis apice terninatis. Dill. Musc.

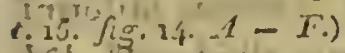

16. c. Jybrida, fronde stipiteque foliacen simplici, ohscure sclyyphifero margine scutellifero', prolifero subulato. Dill. Musc.

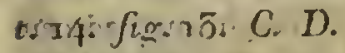

- In Ini exicetis, sylvaticis.

* 17. c. gracilis, fronde foliacea, stipite erecto gracili scyphifero, margine scutel. lifero prolifero subulato. Dill. MTusc. i. 14: fig. 23 .

In ericctis, sylvaticis.

13. c... elangata, stipite .. erecto obscure scyphífero margine elongato, prolifero; \$lıbulato. Iacq. Misc. 2. $\ell_{\text {.11. }}$ f.1. (Lich. elongatus.)

- In montosis subalpinis. (Tyrol. - Colore ci. ncreo, dilute castanco prioris, ..magnitudiue et crassi. 
crassicie diverda. Margo in scyphos vel corni. cula elongatns, saepe stipitc longiora, denticula.. tt18 etiam, et scutellis prolifer.)

* 19 co racliara, fronde foliacca, stipite: erecto incano superne dilatato, margine: cormiculato, simplici proliferoñue. Dill. Musc. t. 15. fig. 16. A. B. Sclirel. spicil. 1114. (Lich. radiatus.)

In ncmorosis, ericetis, pntridis trumcis. ${ }_{c}$ (ac. currit: stipitc elongato gxacili, nargine coriul. bus simplicibus vel scutellatis radiato. Dill. Z: c. $D$; stipite crassiori ventricoso masgine cor. mubus Lnacqualibus, sacpe simplici radiato ; pite subulato simplici, vel mo "alcórove laterali cormu instructo; stipite superne tıi-quadrifitcato. - c. polyceras, stiplte erccto sesinplici ramoso - corniculato scutelliformibus tuberculis prolifero. Jill: l. c. C. $E$. c. furcellnto radisia, stipite erecto maximo siuplici fúcellato ramoso subulato cylindrico prolifero scutellato. - Stipite crassiori rentricoso practer Farinaia candidan squanulis foliolosis recto. Dill. l: c. G.)

*20. c. deformis, fronde foliacea incana; btipite venticoso subulato, scpphifero.

In sylvis montosis, lignis putridis. (Hercyn. - Colore sniphureo, crassitie serounciali, alti: titdine 
indine ferc binnciali; etiam Upsaliae lectam ha. bco; scyphi ampli margine scutcllis obscure coc. cincis vel fuscis obsito. H. 1. Liv. flor. lappl. c.11. fig.5. statira minore superficic ex rufe-" scenti incaua quasi tomentosa, margine dentieulato, contracto; H. I. Dill. Musc: $\iota .15$. Js. 38.)

drarcpe astis: Scyphiferae.

21. c. pyxidata, fronde foliacea, stipito in'scyphutim distiuctum dilatato.

-"In' ericetis' montosis (sequenti occnrrit for$\min :\{x$

- ros exilis, fronde folincea, stipite crecto Hrevissimo scyphifero. Dill. Musc. t. 14. fig. 1.1.

20,5u-ericetis sylvaticis, arborum trmncis, saxis.

* ${ }^{\prime}$ : 'simplex fronde foliacca, stipite ere. cto scyphificro margine integro crenulato. Bill. "Musc. 1. 14. Jis. 6. A. Vaill. paris: t. $21 . \sqrt{\lg g}, 7,8$.

In ericetis. (Margo hinc inde winutis Suscis sciutellis ơbritus.)

* c. incana, fronde foliacea, sipipie erecto scyphifero, incano. Dill. Musc. t. 14. $f . R$ :

Ia ericetis. (Magnitudiue protut actate varia. bilis, margine crenato, scutellato; prolifero:) 
* c. tubaeformis, fronde foliacea, istipite erecto gracili scyphifero. Dill. Musc. C. 14. fig. 10. Vaill. par. t.21. fig.6.

In cricetis montosis.

*.c. fimbriata, fronde foliacea, stipite ere. cto scyphifero margine dentato fimbriảto' simplici proliferoque: Dill. Musc. t. 14.7 fig. 8.

In cricctis montosis.

* c. verticillata, fronde foliacea", stipite erecto scyphifero, disco vel maxgine bis. ter, quaterve prolifcro. Dill. Musc. t. 34 y fig. 6. D. H. Vaill. par. t.21. Jig.5.

* c. prolifera, fronde foliacea, stipite erecto scyphifero disco vel marginc prolifero aggregato. Vaill. paris. 4.21. fig.9. Micho Gen. t.42. fig. P.

In cricetis cum priori.

22. c. tuberculosa, fronde foliacea, stipite erecto scyphifero margine scutellis fus. cis prolifero. Dill. Musc. io 14. fig.6. C. Traill. paris, t. 21. fig. 11. 
*c. marginalis, fronde foliacea, stipite erecto scyphis margine vel disco scyphiferis scutellatis prolifero. Dill. Musc. t. 14 . fig. 6. $I-M$.

- In ericctis sylvaticis, saxosis cum priori.

*23. c. coccinea, fronde foliacea, stipite erecto scyphifero, scutellis sessilibus, substipitatis coccineis. Dill. Musc. t. 1/4. fig.7. In ericetis, sylvaticis. (Occurrit: stipite squa. moso margine prolifero uberculoso Dill. $l . c$. $\varkappa^{\prime 2} L^{\prime}$; centro prolifero scyphifero tuberculoso Dill. l. c. M.; margine producto tuberculosn vèli novis scyphis tuberculosis prolifern, stipite longiori ct crassioni : c. extenss. Vaill. paris. e. 21. fig. 4.

* 24. c: phyllophora, fronde foliaced, stipi. ic érecto in toliola desinente scjphulis minitis obsita. Ehrh. crypt. exs. 28\%. (Lich. phyllophorus.) Vaill. par. t.21. jig.5.

In ericetis sylvaticis putrescenti ligno. (Scy. phuli e foliolis quibus superne cinctus stipes, orituntur.)

25. c. foliacea, fronde expansa foliacon multifido-laciniata adscendente scyphis verrucisque obsita. Mich. Gen. t.42, fig. 2 -2, 
In apricis, inter IMuscos. (Scypli risriast magnituduse, margine integri, cremulati, fim. briati. Verrucae sessiles in superficie frondis. H. vid. 1. Lich. foliaceus Huds. Schreb. spicil. 1115.)

26. c. criscate, fronde foliacea, stipite: erecto scabriusculo obscure scyphifiero, margine foliolis crispo prolifero ramoso subfuscis scutellis cristato. Dill. Miusc. t. 14. fig. 9 .

In ericetis. (Occurrit: stipite hinc inde folio Lis tecto sescumciali, minori unciali, mare्gine. foliolis vix conspicuis, cristato, Dill. l. c. $\mathcal{B}$. Etiam centro hinc inde majoribus scutellis pro.. lifero.)

27. c. turgida, fronde foliacea, stipite: erecto perforato vel obscure, scyphifero ra-. misque turgidis fastigiatis truncatis apice: brevissime ramulosis denticulatis fuscis.. Ehrh. crypt. exs. 297. (Lich. turgidus.)

In ericetis montosis (Comit. Schwartaburg: Rudolstadt Feyder. - Quoad habitum cras. sori biunciali similis.)

-28. c. digitata, fronde foliacea, stipito: xecto incano, cylindrico, simplici ramo.. sóque: 
soque scyphifero: cylindricis ramulis digitato - pulmato. Dill. Musc. t. 15. Jig. 19. A. $B$.

In -ericetis montosis. (Fines ramulorum th, berculatis scutellis minutis coccineis obsitae.)

29. c. squamosa, fronde foliacea, stipito erecto foliolis multifidis squamoso, super. ne ramoso obscure scyphifero.

In moutosis sylvaticis. (Sequenti occurrit forma:)

*. attenuata, erecta, axillis dilatatis ramulis furcatis attenualis stellatis. Dill.' Musc. t. 16. fig. 22. $H$.

* c. denticollis, erecta perforata, ramulis truncatis denticulatis.

c. irregularis, erecta perforata simpli. ciuscula, superne ramosa: ramis iuaequalibus scutelliferis. Mich. gen. t. 12. Orib.g. Dill. Musc. t. 15. fig. 20 .

In ericetis, truncis putridis,

* c. coronata, crecta perforata simpliciuscula, superne ramoso-coronata scutellifera. Dill. Muss. 1. 15. fig. 19.

Cum prioxi. 
3o. c. dilatata, fronde foliacea, stipite - gracili erecto nudo superne dilatato radia. to scurellifero.

In ericetis.

51. c. ventricosa, fronde foliacea, stipite - erecto supenine dilatato ventricoso maximo margine prolifero scutellifero. Dill. Muse. c. 15. flg. 17 .

In ericetis montosis.

\section{Aggregatac.}

32. c. polyesphala, fronde foliacea, stipite squamoso-folioloso erecto, superne dilatato scutellis atro - purpureis aggregatis tecto. Vaill. paris. t.22. Jog. 10.

In ericetis.

53. c. macilenta, fronde foliacea, stipite cylindrico erecto margine scutellis coccineis tecto. Ehrh. crypt. exs.267. (Lich. macilentus.): Mich. gen. t.42. fig.1.2.

In cortice putrido, exicetis, (otipite superne ramoso vel simplici, scutellis lacte vel satura. tins coccineis.)

c. $F i$. 


\section{(IIMXENES.)}

c. Fibula, fronde foliacea, stipite cylin. drico erecto incano, superne dilatato truncato, scutellis subfuscis clauso. Dill. Muse. . 2. 15. fig. 15.

In ericetis.

34. c. fusca, fronde foliacea: foliolis laciniatis minutis imbricatis, stipite cylin-" drico scutellis fuscis aggregatis terminato. Envin. Lich. t.8. fig.4. (Tubercularia ful: sca.) Ehrh. crypt. exs.257. (Lich.. syn!. phycarpus.)

In exicctis.

*35. 'c. parásitica, fronde stipitequé foliofis crustaceis albidis minutis tectis, scutellis terminalibus fuscis. Enum. Lich. t.8. fig.5. (Tubercularia parasitica.)

In tersa, arbornm trmucis, lignis purtidis. (tam mudo oculo foliola distinguenda, ad len.. tern laciniata lincari - multifida. Stipes simplex vel racematim divisus; scutellae nuberculosae subfuscae, fuscae, nigricantes. Occurrit etiam. foliolis obfcurioribus leproso farinaccis crustae. formibus maguitudine et aetate vix a priori di. versus Lich. delicatus Lhrh. cropte. exs. 247.) 
36. c. botrytes, fronde foliacea, stipite lineari simplici superne diviso, scutellis globosis magnis terminato. Hag. Lich. t. 2. Jig.9. (Lich. batrytes.) Jucq. coll.4. t. 4. fig. 3. (I.ich. botrytes (T'ulf.)

- In sylvaticis ad radices Abiesum. (Pornes. Hagen, Carinth. Wulfou, Hanovize D. Meyer. Exemplaria mea ex Insula Montsertret accepi. Scutellae dilute oclureae vel ex luteo rufescentes. Stipes gracilis.)

*37. c. cornucopioides, fronde foliacea adscendente convoluta sinuato-laciniata pinnatifida crispa scyphifera: scypho folio breviore, margine scutellis rufo-fuscis aggregatis tectn. Mich. gen. t. 42. fig. j.

In ericetic sterilibus, ad tcrram, saxa. (H.). Lich. endivifolius, foliaceus contorus snperie Aavo - virefecns, inferue albidus, tuberculis mar. ginalibus rufis Dicliss. fasc. 5. 17. Bacomyces cespititins I'ers. in An. 6ut. 7. 255. Michelii citata figura, excepta maguitndiuc majori quam in meis exemplaribus, non spernenda.)

38. STRROCAUIN (Korallentechte) $G$. pl. 1668 .

Frondes caulescentes tcretes solidae. Scu. tellae tuberculosae, glovosae. 


\section{Tuberculosae.}

* 1. Sт. Corallina, crustaccum tartareum iutus obscure ramulosum: ramis fastigiatis confertissimis teretibus; scutellis ubercuIosis nigris. Enum. Iich. t. 4. J1.g.2. Jacg. coll. 2. t. 15. (I.ich. corallinus $l / T$ ulf.)

In rupibus, saxis. (An huius etiam loci: Lich. uberculosur Enum. Lich. t.2. Jir. 1. ? Isidinra, TVestringi s/char. in Kongl. Acrd. Tel. IJandT. 25. t. 6. f. a.? Lich. oculatm Dicks. fasc. \&., ., 6. Jig.c3:? - In meo corallino obfirfo ramulos ubtusos apice hinc indo fuscos, post lapsum fo. reolas rclinquentes. Scutellae nigrae convexue iil superficie crustae, infrequentes.

2. śr. madraeporiforme, crustaceum taxtareum, 'intus obscure ramulosum : ramis nodulosis fastigiatis obtusis candidis.

In rupibus, saxis, priori inmixtum.

5. \$т. corallinoides, crustaceum atro-cinereum ramulosum; scutellis convexis nigris.

In saxis nupibus, liguo. (H. 1. Lich. niger Huds., quoad apecinina a cel. D. Smilh mecum benevole cormmicata. Nudo oculo et in prima aetate nigrae (in humido pannosac aterrimac) maculae, ad lentem innumeris tectae ramulis 
fubcinereis, sensim erectis confertissimis; scu. tellac depressac, demus convexac.)

4. sт. quisquiliare, crustaceum allidum teuerime fruticulosum; scutellis confertis tuberculosis lateralibus atro - fuscis. Mich. Gen. . 55 . fig. 8 .

In rupium cavernulis, muscis intermixtum. (Nassov. Leers, herb. $99^{3}$, Lich. quisquiliaris.)

5. sт. confine, fruticulosum ramosum: ramis teretibus incrassatis nigris. "flor. Dan. t.879. fig.2. Deutsches gemeinniila. Mag. Tol. 2. t.25. Jig. 5. (Lich. confuis IVull.)

In rupibus ad terram, arborum radicem (Frañ. -on. - Vix semipollicare, ex minutissimis; fruticuli rigidi fragiles, apice hinc inde altc. nuati.)

* 6. sт. condensatum, crustaceum foliolosum adscendens fruticulosum solidum: ra. mis brcrissimis condensatis scutellis immixtis nigris tumbinatis.

- Tn ericeris, montosis. (Quoad foliola tartarea ab initio errac instrata sequenti simile.)

*7. sт. paschale, futiculostum solidum crusta foliolosa tectum scutellisque tuber. culosis 
enlosis ab initio marginatis subfuscis. $P l$. Lich. t.5. fig. 1. (Coralloides paschale.)

In ericetis montosis. (In saxis variat canle ct ramis brevioribus simpliciusculis; cujus exemplaria ex monte Vesuvio Lavae inuasceutia de. beo liberalitati Ill. Schoepf.)

\section{Globifera.}

*8. sr. globiferum, fruticulosum solidum xamosum: ramis diffusis globiferis, latere ramuliferis. Pl. lich. t.51. Jig.2. (Corall.' globiferum.)

ach In montosiz ericetis saxasis. (Hercyn. - Scu. tęllae globosae, juniores clausae; seminta intra stupam filamentosam; adultiores apertae, nigro. pulvere, a seminibus alienissimo, repletae. Affi. niiratern ci transitum praecedeutium Lichenum. cum his ostendunt: Lich. aggregatus et ramulosits cel. O. Swartz, gen. 142, quoad canles or foliola globifero et fragili simillima, scutellis: vero Lich. paschali.)

*.9. ST. fragile, fruticulosum solidum ramosum: ramis fastigiatis, globiferis solita. riis. Pl. lich. t.33. fig.3. (Covall. fragile.) Fl. dan, t. g6o. (Licl. globiferus.)

Iisdem in locis cum priori. (Lich. melausp. orqunes Sivartz, gen. 147 convenit scutellis glo. 
boris apertis incus acro tomentosis; caule foliolis. que subcompressis differt a nottro.)

- 39. usnea (Haariechte) G. pl.s668.

Frondes loreac vel filamentosac. Scutel. lae' xadiatac aut nudac.

Articulatae.

*. v. plicata, loris pendulis ramosis implexis; scutcllis radiatis. Dill. Mrusc. C.11. fig. 1 .

Iu sylvis, ad arbores (Hercym.)

* 2. Tarbata, loris pendulis ramosis fi. Iamentosis: flamentis patentibus. Dill. Musc. t. 12. fig. 6.

In sylvis, ad arbores. (A priori rix specie diversa. In uncis exemplaribus, in variis Luropae locis collectis, lora uunc crassiora nunc tenuiora, ultimo filamentosa implexa; ad latera fila. mentis transversalibus obtecta. Superficies punctis exaspernta, hinc inde transversin dimosa, in articulos quasi seceleus, pro more hujus sub. divisionis, Glo trajecio conspicuo. Scutellae nt In priori sparsae, orbienlatac, planae, margine radiasac. Inter has aliac occurrunt minores tuberculosae mfescentes, carmosac in humido, snarginc undze, vel subtts ex flamento prode. rute radiatac.) 
75. v. hirta, loris erectis ramosis filamentosis; scutellis nudis. Pl. lich. t.3o. fig. 1. Dill. Musc. t.13. Sis. 12.

Ad corticem arborum, dumeta, sepes, saxn. (Scutellac rufescenter, tuberculosae, margiue nudae, ab initio leniter excavatac, similes prae. cedeutis Usueae, longe infrequentiores quam in sequeuti.)

*4. U. florida, loris erectis ramosis fila: mentosis; scutellis radiatis. Il. lich. t.5o. fig. 2. Dill. Masc. t.15. fig. 15.

Ad corxicem arbornm, ramos, virgulta cum priori. (Variat sc:ttellis majoribus et minoribns, etiam miuntssimis radio conspicuo cinctis.)

5. U. articulara, loris articulatis articuJis incrassntis; scutellis nudis. Dill. Musc. 1.11. $\operatorname{sig} 4$.

In sylvis ad arhores. (In monte Dino non louge Gissa dissito Dill. Palatin.? Nassov.? Bo. rinss.? Cariuth? - Etiam in arenosis collectam habeo. Scutella in exemplaribus meis in Italia collectis tuberculosac, similes iis, quat $M T i c h$. in gen. . . 59. Jg. I. cxpressit; aliäe orliculatae Int12c.)

* 6. v. Saccicia, loris pendulis Maccidis. conpresso-lacunosis. Dill. Musc. t. 12. f. 5. 


\section{C CPXPTOGAMIA SCUTEILATA}

In aluerum cortice. (Jercyn. IVeb. s)icil. 227. I.ich. divaricatus. In comit. Schwartzburg littdolstadt IIeyder. Fotiam in arenosis. H. 1. Tich. arcnarius, fruticulosus rugosus ramosissimus candicaus, ramis diffusis 7 eig. Relz. scalld. cd. 2. 1642.$)$

\section{Inarticulatae vel filamentosae.}

* 7. U. dichoroma, loris pendulis dichoromis compressis glabris, filamentosis, oclrolencis.

In montosis sylvaticis. (Hercyn: Mhrh. Frondes pedales dichotomo-multilidae z̧abrae, ultimo capillares, pallide e cincreo Aaventes: crassiores rami compresso - lacmosi; in siccitat rigidiusculi. Ad lentem apices filamentoxum ni. gricant. Dill. musc. t. 15. Jis. 15́., bene, quoad formam, nostram exprimit, in descriptione colo. ris obscure lutei ad ruffum tendentis, pre se. nium forsan mutati, differcutiam monstrat. Fitiam ex Alpintis Norregine accepi curn nomine Lich. rigidi Villarsii.)

* 8. U. jubaia, filamentosa pendula, axil. lis compressis. Dill. Musc. t.12. fig.7.

In sylvis ad arbores. (Praeter cincreum oc. currit ad saxa et cortices arborum colore fu. sco, nigricante: v. implexa, filamcutosa de. cum. 


\section{(IICHexss.)}

cumbens implexa, filis longis diraricatis simpli. cinsculis. Filamenta crassiora in tubercula $\mathrm{fa}$. sinosa effloresciunt.)

* v. ramulosa, filamentosa implexa pendu. Ia dichotome divisa, filis apice rel latere ramulosis multifidis cinereis. .

In sylvis ad arbores (hinc inde cum prior?, colore ex ochreo vel llavescenti cinerco.)

* G. v. bicolor, filamentosa erectiuscula, inferne migricans opaca, superne dilutior fusca nitida. Elurh. crypt. exs. 40.

- In sylvaticis montosis, ad arbores, saxa. (Her. cyn. $\mathbb{E} 7$ rr $_{2}$. Beitr. 5. 32 ; Lich. bicolor, filamentosiss, ramosissimus, erectiusculus, teres, inar. ticulatus, glaber, nitidus, inanis, infra nigricans, supra'sordide albidus, intus griscus: rn. mis patentissimis: extremitatibus sinplicibus, subulatis: - Gott. Heyd.)

* 20. U. chalybeiformis, filamentosa decumbens simpliciuscula vage rimosa llexuosa. Dill. Miusc. t. 15. fig. 10.

Ad cortices arborum, saxa, scpimenta.

11. v. larea, filamentosa decumbens ramosissima implexa fusca nitida. Ehrh. crypt. exs. 70. (Lich. laneus.) 
In monto:is ad saxa, arbores. (Fila divari. cata inplexa ramosissima terctia, ultimo multilida, furcata, fusco - nigricautia, rigidiuscula. A priori dcfectu nitoris et colore nigricante di. stiugui potest:

* V. lanata, filamentosa decunibens ramon rissima implexa nigricaus opaca.

In wontosis ad rupes, snxa. (Hercyu: inter Lich. Fahlun.)

42. v. puboscens, flamentosa decuinbens ramosissima implexa alra, scutcllis, plauis concoloribus. Jaeq. Misc. 2..t.g. fig.7. (Lich. pubescers $M /$ ulf.)

In montosis (Hercyn., scutellae atrae nitentes,. per lentem radiatae; Cariuth., scutellse magis integrac. A priori flamcutis tenuioribis con.. fertiscimis atris et ramosis, ctiam absque scutellis distinguitur. Ab utraque subtilitate byssacea। fere differt:

13. U. intricata, decumbens implexa ca-. pillacea atra; scutellis planis concoloribus. Ehrh. crypt. exs. 8o. (I.icl. intricatus.)

In montosis, rupibus. (Exemplaria mea Upsz.. liae ab Fillharto lecta, ad Ientem lila transver.. aim sugulosa habent, ut in Lich. scabro Iruds.) ' 
* 14. U. hippotrichodes, fulamentosa siru. plex capillaris. Web. spicil.251. (Iich. lipporrich.)

In foliis dejectis acerosis. (Non rara per om. nes sylvas Germaniac. Aliam in Ilercyn. montoris et subterraneis legi craesioribtis filis sub. compressis, quibus corpuscula adnascuntur ovala, intra substantiam corpusculis 6eminiformibus ad Ientern funcis oblongis plena, qualew indicare videntur: Dill. musc. r. 3. Jig. 12. 13.; Lich. seioszs, filameztosus simplex subcompresulus mi. Gicans,' mberculis globosis acuminatis nigrie Teys. hal. 2. 285.; Hypoxylou Loculiferum, call. lescens nigrum, super ramib criuiformibus sparsps.gerens locellos Bull. 7eerb.)6. 495. Jig. 3. p. 74, quoad fructilicationem certe a Licheumm ordin. alienam.) in ,

\section{Fruticuliformes.}

* 15. U. ochroleuca, erecta teres dichotoma ramosa: ramis divaricatis filamentosis furcatis apice nigricantibus.' P. lich. t.26. fig. 2 .

In montosis (Hercyn. Siles, - H. 1. Lich. subvirescens Gmel. Syst, Nat. 1878. Lich. flx." vidus i६. 3579 )

16. $\leftarrow$. 
16. U. vulpina, compresso- lacunosa ramosa dichotoma fastigiata cirrina. Jacq. Misc. 2. tab. 10. fig.4. (Lich. vulpinus II ulf.)

In sylvis acerosis montosis. (Carinth: IVulf. Sehrank, havar. 2. 2560. Iich. citrinus? Iu muris, tectis, sepimentis Ducat. 3rem. Roch, germ. 1. 516. - H. 1. Lirh. cirricolorus Gmel. syst.nat. 1379. Dill. musc. t. 23. fig. 15. Hall. hist. 1974, quoad viva Exemplaria Anctorum, magnimdine unciali et binnciali. Ad lentem puncta nigra inter farinam sparsa ob-crvo. Scu. tellae in hac et priori adhuc desiderantur.

\section{IOBania (Vieltheilige Flechte) $G$. pl. 1668.}

Frondes lobatae, laciniatae, multifidae, erectae, depressae. Scutellae marginatae.

$$
\text { (YLA T IS II A :) }
$$

Frondo lacunosa erocta.

1. I. fraxinea, erecta laciniata compres. -a nuda; scutellis undique sparsis subpedunculatis. Pl. Lich t, 18. fig. 1-2. (Pla. tisma (raxineum.) 
In cortice arborum, Fraxino, Quercu. liteari ad biuncialem latiudinem.)

* 2. I. farinacea, erecta laciniata compres. sa margine rerrucoso - farinacea. Dill. Musc. t.23. fig.65. Vaill. paris., t. 20. fig. $13 \cdot 14$.

- In cortice et ramis arbornm. (Scutellae pal. lidae contolores.)

* I. Squarrosá, erecta laciniata squarrosa coumpressa, fastigiata matgine eroso-farinacea: Vaill. paris. t. 20. $\sqrt{2} g,: 5$.

In corlice ramisque arborum. (Scutellae ut in priori, terminales.' H. 1. Lich. squarrosus, cespilosus ramis confertis - Raccidis multifidis," crispis, latere subfarinaceis; - scutellis urceolatis. concoloribus. Pers. in All. bot. 14. 35.)

* 5. I. calycaris, erecta laciniata compres. sa. nuda rigida; scutellis subtus calcaratis. Dill. Musc. t.25. fig. 62.

In cortice Arborum, ruplusus. (Occurrit an. gustioribus laciniis, margine, sed raro, hinc in. de vermcoso-fariuaccis, nigro - macnlatis. H. vid. 1. I.l. dan. 1.959. fig. 1. Iraill. prisis. t.20. fig. 7. Multo latioribus, secuncuti populino si. wilis, ramis véro subter scutellas compressis." Dill. l. c. C. Magnitudo uncialis; palmaris : 
F.l. dan. 7.c. fig. 2., quoad habitum; margo in. eger vel lacinulatus:)

* L. dilacerata, erecta laciniata squarrosa compressa rigida nuda.

In cortice arbonum, saxis (uncialis Dill. Musc. t.21. Jig. 55. G.; quoad habitum, et lat tioribus biuncialibus laciniis, Equarrosae similis, scutellis subtus obscurius calcaratis.)

* 4. I. populina, erecta cespitosa laciniata: laciniis superne dilatatis turgidis fasti. giatis. Ehrh. crypt. exs. 276. (Lich. populinus.)

In cortice arborum, Populo, Salice. (Scu. tellae coucolores terminales, subtus undae vel calcaratae turgidae. (E Hispania Fico indicae adnascentem similem plantulam attulit ill. Prof. Abildgaard, mecumque communicavit, in qua scutellae tenui uigricante margine cinctae sumt. $\rightarrow$ H. vid. 1. Dill. Musc. t.21. fig. 55. $I, G, B$. Faill. paris. 1.20. fig. 22. 1.21. fig. 2. Lich. fat stigiatus, cespitosus ramosus: ramis sursum dilatatis fastigiatis inermibus: scutellis subtermi. ualibus Pers. in An. bot.7. 256.)

* 5. I. prunastri, crecta laciniata compressa subtus incana, margine verrucoso-farinacea. Dill. Musc. t.21. fig.55. A. (Lichenoi. 
chenoides cornutum bronchiale molle sub. tus incanum.)

In truncis ranisque arborum, fruticum. (Scutellae rufescenti - fuscae, extus, couvexac frondi concolores. In aliis individuis ascutellatis ob. servo tubercula conglomerata nigro - fusca. Oc. currit laciniis utrinque nudis concoloribus angust is, scutellis amplis, $\mathbf{K}_{0}^{*} 1$ : Licl. corniculcs. tus, foliaceus erectiusculus sublacumosus glaber, laciniis angustis corniculatis Iielh. cantabr. 453. Dill. Miusc. 1.21. Sig. 54.)

6. I. denudata, erecta laciniata Iacerata compressa; scutellis deuudatis terminalibus fulvis. $\mathrm{Pl}$. lich. t.5̃. fig. 1. (Platis. ma denudatum.)

In cortice Tiliae. (Palatin. rarissimam plau. tam collegit recumque communicavit Gaertner jur. Hauoviensis, exquisitus plautarum cultor.)

* 7. I. tinctoria, Erecta subconupressa la: ciuiata: laciniis auastomosantibus, ultimo inrcatis liberis fastigiatis farinaceis. VVeb. spic. 241. Ehrh. orypt. exs. 266. (Lich. inctorius.)

In montosis saxosis (Hercyn. Web. prope Nordhauseu, Blankeuburg L7er/e. Scutella* frondi concolores; lunc inde ad lentem nigra 
sparsa funt puncta per superficicm froudis, in sicco rigidae; aqua calida perfusa purpurascen. tc statim tiugit colore.)

*8. L. rigida, erecta subcompressa adscendens cornea lincari - multifida fusco - nigra: scutellis terminalibus concoloribus. Pl. lich. t.24. fig. 1.. (Cornicularia-tristis.) Enum. Lich.74: (Lich. rigidus.)

In eaxis montosis (Hercyu. Web. spic. t.5. Lich. uistis. E/hrh. - Cariuth. Wrulf. Lich. fucoides. In Jacq. Coll. 5. 1. 42. flg.3. Licl. fuciuus Gmel. Syst. Nat.1378. Scutcllac et la. tiores rami subtus lacumosi. Ob peculiarem cor. neam nauram peculiare genus ex ea 1. c. con. slitui.)

*9. I. aculeata, erecta subcompressa la. cumosa fruticulosa castanea, vamis scutellisque hispidis. Pl. lich. t.5. fig. 2. (Coralloides aculeatum.)

In ericctis, montosis. (Occurrit canle ramis. que crassioribus mudis, dilnte castanea, Islan. dicac minori bimilis. - I. 1. Hich. spadiceus fioth. in Mag. bot.2. t. 1. fig. I.; canle ramis. que tenuioribus: r. tenuissinn, ramosibsima fu. sco nigra, apice spinuloso - furcata. Dill. Musc. t. 17. fig.a 2. Il. lich. l. c. fig. \&, a. Lich. is. land. 


\section{(IICHENES.)}

143

land. nigricans lietz. scraid. 2. 1576 . Lich. is. landicus tennissimus Ehrh. T3éitr. 5. 35. Iiŝdem cum jriori locis.)

* 10. I. islandica, erecta laciniata comi pressa canaliculata: laciuiis bicoruibus scutellisque ciliatis. $M$. lich. t.g. Jig. 1. (I.if chenoides islandicum.) Jacq. coll.4. t.8. (Lich. islandicus If ulf.)

In montosis, ericetis. (Basis sanguinca. Oc. currit latissima palmari fronde, et ingustiore: crispa Dill. IMusc. t. 28. fig. 112. Etiam margi. 1.e paciliata.)

11. L. nivalis, erecta adscendens laciniata, lacunoso - canaliculata crispa albida. Dill. Mlusc. t.21. flg.56. A. Flor. dañ., t. 227. Flor. lap. 2. t. 11 . Jig. 1.

In montosis subalpinis (Tyrol. Siles. Rolen. -; Semper absque scutellis accepi, saepissime cuni sequenti confusam.)

12. I. cucullata, erecta laciniata alba, margine complicata simata crenulata. Bel:' lardi, observ. 5\%. Trans. lin.1. t. 4. fig. 7." (Iich. cucullatus Snith.) Dill. MLusc. t.21.i fig. 56. B. 
In montosis, subalpinis (promiscue cum prio. ri, ejusdemque coloris ex dilue havo vel viri. di candicantis. Scutellae ut in Lich. islandico frondem terminant, dilute rufescentes. Occur. rit angustiore et latiore fronde.)

-13. I. furfuracea, adscendens dichotome laciniata: laciniis acutis, inferne nigris canaliculatis. $P l$. lich. t.g. fig. 2. (Liche. noides furfuraceum.)

Ad truncos arborum. (Sentellae rarissimae, in superficie couvexa ramorum essiles scmicu. pulares 11 in $x$. prumastri intus rufescentes; extus convexae, furfure concolore adsperșe. Occurrit angustioribus et longioribus laciniis, brevioribus et latioribus.)

* 14. r. ciliaris, erectiuscula laciniata: laciniis linearibus inforne canaliculatis margine ciliatis palmatis. $P l$. lich. t. $5 . \sqrt{15} .4$. (Lichenoides ciliare:) Jacq. coll.4. t. 13. fig. x. (Iich. ciliaris MIulf.)

Ad truncos arbornm, taxa. (Scutellac pe. diuculatae crenatae. Color in sicca cinercus, in lumida virescens. Eandem ex America sep. tentr. a D. Muhlenherg accepi fronde utrinque permaneuter albida, scutellis Lividis ciliatis; e Norlandia laciniis angustis fusco - nigricautibus.) 
Fronde lacunosa depressa.

* 15. I. saxarilis, depressa scabra, reti. culato-lacunosa, sinuata; scuicllis rufofuscis. Enum. Eich. t. 15. f. 1.. t.16. f. 1. Jacq. coll. 4. t.2n. fig.2. (Iich. saxatilis t.Tulf.)

La saxis, arboribus. (Frondis laciniis angu* stioribus vel latioribus cinereis, fermgineo-fuscis. I. omplłalodes, diffusn lacunosa glabra nigro - fusca; scutellis concoloribus. 7 illl. praris. c.20. fig. 10. Exemplaria possideo endem a Vail. Jantio collecta loco. Eliam ex Hercyuta. - $\mathbf{X}$. aclusta, diffusa scabra reticulato lacinọsa gra. sulosa ciucren - nigra; scutellis concoloribus. In montosis rupestribus cum priori.)

16. I. sepiricola, depressa adscendens laciniata castanea, subtus reticulato-lacunosa; scutellis terminalibus concoloribus. Pl. lich. t.i4. fig.1. (Platisma sepincola.)

In arboribus, lignis, saxis.

17. I. juniperiua, adscendens depressa la. ciniata crispa flava; sculellis terminalibus fuscis. Pl. lich. 6.7. fig. 2.

(Squamaria juniperina.) $\mathrm{Fl}$. dan. t. 1004 .

In cortice arborum, Junipero. (Nasaov. Hols28.? Thuring-? Hal. Sal.? Cariutlı? - An un- 
quam iu Germania reperta vel potius cium se. queuti confusa? -)

* I. pinastri, adscendens depressa laciniata crispa Aara, narijue pulvere cuncolore fimbriata. Pl. Lich. t.7. fig. 1. (Squamar. pinastri.)

In trumcis arborum piucarum frequens. (Abs. que scutellis.)

* Ig. L. verrucosa, depressa rotunde-lobata scrobiculata verucosa fariuosa; scutellis centralibus rulo-fuscis, $P$. Lich. t. 1. fig.1. (Pulmoutaria verucucosa.) Lácq. Coll.4. t. 18. fir. 2. (Lich. verrucosus.) Vulf. Flor. dan. t.1007. (Lich. pulmona. rius.)

In arborum truncis, saxic, rupiluss. (H. I. Lich. pluubeus Jioth. Gernt. 50\%. In bot. Mag. 2. 2. t. 1. fig.2. Lich verrucarius Ginel. syst. nat. 1356.)

* 20. L. pulmonaria, depressa laciniata simuata truncata reliculato-Lacunosa; scuteilis marginalibus rufo-fuscis. Pl. Licti. ๖. 1. fig. 2. (Pulinou. reciculata.)

In arborum truncis, Quercu, Fazo. (Và. vivipara 1 oth in bot. Mag. l. c. t. 1.ifig. 5.) 
Fronde dilatata:

21. I. herbacen, subimbricata depressa obtuse lobata crenata; scutellis fulvis. $P l$. Lich. t. 10. fig.2. (Pulmon. lobrita.)

In saxis arloribus. (Hamover, in monte Dei. ster Ehrh. - In humido laete virens. H. 1. Lich. laciniosus. Gmel. syst. Nat. 25772.)

* 22. L. saccata, subimbricata depressa obinse lobata; scutellis subtus saccatis. Dill. Musc. t.30. fig.120. Fl. dan. t.531. (Iich. saccatus.)

" In terra locis montosis rupestribus. (Semina intra scutellarum substantiam coordinata, faci. lius quam in ullo alio Lichene conspicienda. Mich. gen. $1 . \varepsilon_{2}$.)

*25. I. acetabulum, subimbricata depressa obtuse lobata; scutellis corrugatis duplica. 10 - marginatis. Enum. Lich. 1. 18. fig. 2. Jacq. Coll. 3. t. 9. fig.1. (Lich, Acetabu: Ium $M$ ulf.

- In cortice arborum. Palat. Neck. Comit. Bemili. Hannover. Kihth. Nassov. Leers. - H. 1. 'Lich. corrugatus Smith; Transact. lin.1. 83. Lich. serratus Gmel. syst. nat. 2372. Lich. pa. tcllatus ib. 1366.) 
*24. I. aquatica, subimbricata depressa plicato. Lobata verrucosa; scutellis fuscofufescenibus. Pl. Lich. t. 45. fig. 2.5. (Platisma aquaticum.) WVot. spicil. 8.4 . (Lich. Ruviatilis.)

Ad saxa intra aquarum rivulos. (Hercyn. Francof. Tieich. flor. 849. - Intra aquam satu. rate vircns.)

-25. x. glomulifera, subimbricata depres: sa simato-lobata glomulifera; scutellis fulvis. Lightf. scot.853. (Licl. glomu. lifer.) Dill. Musc. t. 26. fig.99. Mich., gen. $t .46$.

In arborim truncis Fag. sylv. (Gott. Pers. Lich. grandis An. bot. 11. 13. Palat. Guertno) * 26. r. caperata, subimbricata depressa rugoso-lobata pallide luted; scritellis fus. co.rufescentibus. Pl. Lich. t. 58. 5g. fig. x. 2. 42. fig. 1. (Platisma caperatum.)

In cortice arborum, saxis.

*27. L. perlata, subimbricata adscendens depressa crispato lobata fimbriata verrucoso fariunosa. Jacq. coll.4. t.10. (Lich. per. Latus. $(v$ ulf.) 


\section{(IICHENIS.)}

In cortice arbornm, saxis. (Scntellae subpedunculatae fusco rufae in disco sacpe perforatae.) * 28. I. glauca, subimbricata depressa ad. scendens haciniata crispa. Enum. Lich. e. 20. fig. 1 .

In cortice, ligno. (Priori tenerior, margine laciunlis crispato. Similem plantam, memorabi. Iem propter lacinias frondis angnstiores adscendentes, in capitula inflata abeuntes turbinata, anne monstrosam varietatem dicam nec ne? dubius haegco.)

* 29. x. fallax, diffusa laciniata adscen。 dens crispa; scutellis terminalibus spadiceis. Pl. lich. 4. 46 fig.1-3. (Platisma fallax.)

In cortice Abiet. (Hercyn. Web. spic. 244. Kich. fallax. Lich. frandulentus. Gmel. syst. Niat. 1373.)

*50. I. tiliacer, subimbricata depressa rotunde lobata simuata albido - cinerea; scutellis fusco rufescentibus. Enum. Lich.t. 1 \% fig. 2. (Lich. tiliaceus.) Jacq. coll. 3. t.9. fig. 2. (Lich. quercifolius Wulf.) IVilld. berol. t. 7. fig. 15. (Lich, quercinas.). 
In cortice ramisque Tilize, Quercumm alia. rumque arborum. (Froude pulveruleuta vel nu. d2. H. 1. Lich. Lobatts Gmel. syst, Nat. 1372.)

*31. L. olinacea, subimbricata depressa, rotunde lobata olivacea; scutellis concoloribus crenulatis. Fnum. Lich. t. 15. fig.5.5. (Iich. olivac.)

In cortice arborum, rupibus, saxis. (Occur. xit fromie crispa exasperata scutellifera, vel sterili orbiculata mida. Etiam imuricata foliolis multifidis olivaccis scutellis integerrimis. II.' 1. Licl. pullus scizreb. spicil. 1127.)

* 52. I. parietina, subimbricala depressá. Iobata crispa fulva; scutellis concoloribus. Enuin. Lich. t. 10. fig. 1. (I.ich. pariet.)

In cortice arbornm, lignis. (Var. colore Ilavo, virescenti, fronde sterili pulveruleuta; scue tellis proliferis Humb. frib. 1.2. fig. 2.)

$$
\begin{gathered}
\text { ( } x \text { II s c I A.) } \\
\text { Frinule inflata. }
\end{gathered}
$$

* 33. I. phiysodes, subimbricata depressa, adscendeus laciniata ntultifua inflata, nuagiue delisceus fariunsa. Enum. Lich. t. 15. fig. 2. (I.ich. pligsod.) Jacq. coll.3. 1.8. (Lich. physodes. $W$ ulf.) 
In cortice arbornm', saxis. (Scutellac subfuscac inflato ruguloso pedunculo insidentes. Fron. des teretes, compressae, stbfuscac.)

34. I. terebrata, subimbricata depressa laciniata multifida inflati punctis perforaเ. 'Jacq. l. c. . Eig. sinistr.

In cortice arborum, hinc inde c. priori, red. xarius. (H. 1. Lich. pertusus Schranck havar. 2. 1515. Schrred. spic. 96.).

$\Rightarrow$ 55. I. hispida, depressa adscendens se. mitulumlosa infata ciliata farinosa. . 1l. lich. t. 5. fig. 2-5. (Iichenoides lispid.) Jacq. coll. 4. t.6. (I.ich. hispidus TI ulf.)

In cortice ramis arborum, fruticum, saxis. (H. 1. Lich. semipinnatus Grnel. syst. Siat. 1572.)

\section{( $S Q \cup \wedge M A I \cap \wedge:$ )}

Fronde angustata.

56. I. semipinnata, subimbricata depres. 8a multifida ciliata subeus canaliculata; scu: tellis nigricantibus. Dill. Musc. . 20. f. 46 . $A, B$. Wulf. l. c. $u, b$, c.

- In cortice arbornm; saxis, (orbiculatim ex. pansa $2-3$ Uncias lata, mudique pilis citiatis hispida, quozd colorem et divisionem stellari: formi similis.)

*37. 
* 37. I. stellaris, subimbricata depressa orbicularis multifida: foliolis linearibus; scutellis cinereis, pullis. Enum. lich. t. 13. fig. 2.

In coxtice ramisque arborum, frutictum. (H.1. Lich. ambigums E7w7. crypt. exs. 207. L. stelluri. formis, foliolis latioribus depressis, margino lobatis crenulatis; scutellis fusco - nigricantibus. Ennm. Lich. l. c. flg. 1.)

58. I. aipolia, subinbricata depressa orbicularis multifida: foliolis laciniatis loba: tis cnurexis; scutellis cinercis integris, fu. sco-migricantibus. Enum. Lich.t.12. f.2. C. Ehrh. cr3pt. exs. 197. (Licl. aipolius.)

In cortice arborum. (scutellae in sicco quasi pruinatae.)

* $3 g$. I. pulverulenta, subimbricata depres. sa orbicularis multifda: foliolis lobntis li. ucaribus nlutusis crenulatis apice impres. sis, pruinalis. $1 \%$. lich. t. 8. fig.2. (Squamaria pulreruleuta.) .

In arborune cortice, saxis. (Color in sicco cincreus, glaucus, aetate fuscus, iu humido viresccus. Scutellae jumiores $n$ gramule per su. períciesn sparsae, integrac vel cresulatae polli- 
ne conspersite, demum fusco, nigrae, H. 1 . Lich. omphalodes Wrulf. in Jacq. coll; 2. 4.15: fig. 2. a.b. Lich, allochrous. Ehrte. crjpt. exs. 187. Occurrit foliolis multifidis linearibus difii: sis scutellisque pruinatis: Li angustata', Erum? lich. t. 11. $\sqrt{i g} .2$.

* 40. I. pulveracer, subimbricita depressa orbicularis multitida: foliolis margine crispis pulveraceis, apice lobatis obtusis crenulatis impressis pruinatis. Ehrh, crypt. exs. 285. (Lici. pulverulentus.)

In cortice arborum. (Scutellae rarins occur. ruut hitc inde sparsae aeque pulveraceae. IMu. scis instrata, centrum versus penitus pulver gramuloso cinerascenti, caesio tecta.)

41. I. speciosa, subimbricata depréssa suborbicularis multifida: foliolis alte divi. sis linearibus obtusis sinuato - crenulatis apice impressis; scutellis hemisphaericis: Jacq. coll. 3. t.7. (Iich. speciosus Wulf.) In rupibus, super IMuscos (Carinth. Wulfen -s Eandem ex America sept; accepi a D. Muhlem. berg. - Scutellae in disco rufescentes, margiue introllexae; foliola margine pulverulenta vel nuda, in sicco ex albido cinerea, in recenti vírescentia, ciliis margine' prominulis.) 
* 42. L. pulla,' subimbricata depressa multifida : foliolis subteretibus linearibus apice dilatatis fuscis, multipunctatis; scuicllis rufo-fuscis. Pl. lich. t.32. fig.2. (Squamar. pulla.) Transact. lin. 1. 4.4. fig. 6. (Lich. encaistus Smith.)

In rupibus montosis (Hercyn. - Efirh. crype. exs. 505." Lich. multipunctus. Foliola intus so. lida, apparcut iurgida convexa. Lich.' fuscus Husds. diversa et caute distingnenda plantá.)

* 43. x. centrifuga, subimbricata depressa imultifida: foliolis adpressis centrifugis yiridantibus; scntellis rufo - fuscis. Pl. lich. t. 16. Sig. 2. (Squamar, centrifuga.)

- In saxis, lignis.

44. x. stýgia, subimbricata depressa multifida: foliolis palmatis recurris nigris. $\mathrm{Pl}$. licl. t. 25. fig. 2. (Squam. stygia.)

In rupibus, saxis (Hercyn. $\rightarrow$ )

* 45. r. fahlunensis, suhimbricata depressa multifida: foliolis dichotomis canaliculatis nigris. Pl. lich. t.36. fig.2. (Squam. [ahlun.)

In rupibus axis (Hercyn. $\rightarrow$ 


\section{(PIACODIUAI:)}

Fronde crustaced.

* 46. I. ciliara, subimbricata suborbicula. ris drpressa multifida: foliolis laciniatis scutellisque subtus ciliatis. Enum. Licho t. 1 / . fig. 1 .

In cortice arborum. (Occurrit foliolis angut. stioxibus vel latioribus, cinereis, fusco - nigri-? cantibus, margine integris vel verrucoso. pulot verulentis; scutellis plus nninusve unbens cilia. tis, fusco nigricantibus, nigris. H. L. Lich. ob. scitrus Ehr/. cropte. exis. 277.)

* 17 . x. orbicularis, imbricata depressa cinerea, foliolis lobato-multifidis margineelevatis pulverulentis. Eutm. Lich. t. 9. fir. 1 .

'In cortice arborum (promiscuè c. priori et $L_{\text {. }}$. candelari.)

* 48. x. ambigua, diffusa subimbricata de -: pressa: foliolis lineari-multifidis flavo. virentibus, tuberculis concoloribus; $8 \mathrm{cu}$. tellis subfuscis. Pl. lich. c. 40. fig. ? : $4 \cdot$. (Psora ambigua.)

In cortice Abiet. lignis. (H. T. Lich. diffnsus IVeb. picil. 250. Colore occurrit in cincreura vergente. 


\section{CRYPTOCAMTA BCUTEILATA}

* 49. I. diffusa, subimbricata suborbicu. Jaris depressa: foliolis lobato.multifidis farinosis crispis subcinereis; scutellis elevatis ferrugineo-fuscis.

In cortice Abict. Palis, scpimentis. (Margo scutellarnm crnsta concolor. H. 1. Lich. diffusus Dicks. fasc. 5. 4.9. f.6; excluso syu. Web. - L. pallescens. Orbicularis centrifuga rugo. so mulifida cervina farinosa; scutellis promi: nulis subfuscis. Enum. Lich. t. 10. Jis. 1 - 2.)

5o. I. Iubia, subimbricata orbicularis depressa foliolis lizveari-multifidis albidocinereis, centrifugis pulverulentis nigris. Enum. Lich.70. (Lich. diffusus.)

In tectorum tegulis, saxis. (Stellari vel cacsio quoad folia similis.)

* 51. I. caesia, subimbricata depressa orbicularis multifida: foliolis albido - cine-: ris; scutellis tuberculisque caesiis. $I$. lich. t.8. fig. 1. (Psora cresia.)

In saxis, tectis, lignis, Muscis. (H. 1. Lich. pulchcllus Wulf. in Jacq. Coll.2. t. 16. Jig. \&, Iich. Psora Dicks. fasc. 3. 17.)

*52. x. incurva, subinbricata depressa orbicularis multifida: foliolis semiteretibus viridantibus apice incurvis.

Is 
In saxis. (H. 1. Lich. incurvus Pers. in An. bor. 7. 2., Lich. multifidus Dicks. fasc. 3. t.9. fig. 7. Tubercula pulverulenta, scutellae fitscre.)

* 55. . radiosa, subimbricata orbicularis depressa: foliolis lobato-multifidis radiantibus cinereis; scutellis confertis, fusco. nigris. Enum. Lich. t. 4. fig.5. (Iiclı. radinsus.) Achar. in kongl. Vet. Acad. n. Handl. 15. t. 6. fig. 5.

In rupestribus, saxis. (H. 1. Lich. ubimbricatus Relh. calte. 427. C. ic: Lich. circinnatu Pers. in An. bot. 7: 25.)

*54. t. versicolor," subimbricata orbicula: xis depressa : foliolis lobato-multifidis, pallidis, viridantibus; scutellis confertis subfuscis.

In rupestribris," sáxis. (H. l: Liclı. versicolor foliis tartareis imbricatis dilatstis, virescente. cinereis albidisque; scutellis parvis confertis? planiusculis subrufis. Pers. in An. bot. Z. c. $2 \xi$.

*55. I. muralis, subimbricata orbicularis depressa: foliolis lobato - multifidis concoloribus, scutellis confertis subfuscis con.

$$
\text { L } 2
$$

stantes 
stanter marginatis. $P l$. lich. t. 16. fig.1. (Psora muralis.)

In muris, eaxis, tectis, lignis. (H. 1. Lich. ochroleucus IFulf. in Jizq. coll.2. 1. 13. fig. 4.)

*6. I. saxicola, subimbricata suborbicu. laris depressa: foliolis lobato-muliffidis pulposis luteis scutellis concoloribus. Pl. lich. t. 17. fig.3. (Psora saxicola.) Ezzmm. Lich. t.9. fig.2. (Lich. murorum.)

In muris, saxis, lignis (foliolis integris, pul. verulentis centrifugis, scutcllarum disco conco. lore vel aurantio. - Etiam in tectorum teglllis, nupibus, colore pallido, virescenti luteo, fulvo, aurantio, mistiato, h. 1:)

* tegularis, orbicularis subimbricata de. pressa: foliolis lineari - multifidis pulposis aurantiis, scutellis concoloribus. Elartso erypt. exs. 5ol. Il. lich. l. c. a.)

*57. I. mininta, crustacea sparsa suborbi. cularis: foliolis minutis scutellisqne miniatis. Enum. Lich. 62. (Lich. miniatus.)

In ripibus, cum prioti, saxis arenosis, (Nit. dis oculis foliola minutissima vix a scutellis di. stingueuda, iisque saepe oblitterata.) 
* 58. x. candelaris, crustacea depressa : fo. liolis minutis multifudis confertis flavis pulverulentis; scutellis margine concoloribus. Enum. Lich, t.g. Jig.j.

In cortice arbormm, saxis. (Foliola vix nisi ope lentis couspicienda; Scutcllarum discus unargine concolor, vel livescens. Sterilis occur. rit confertissimis foliolis pulverulentis parum divisis. Sinilem ex America septentr. a $D$. Nuhlenbers accepi plantnlam clegantius constructam, foliolis multifidis scutcllisque albido. ciliatis.)

* 5y. I. concolor, crustacea subimbricata: foliolis miuntis congestis brevissime lobatis Rarescenti - aurantiis crispis; scutellis concoloribus. Dicks. fasc. 5. t. 9. $\sqrt{\mathrm{g} g} .8$. Enum. Lioh.59. (Lich. flavesceus.)

In arborum cortice, ligno vemsto. (Ad len. tem foliola saepe pulver ulenta obliterata. Ia fulva, subimbricata depressa: foliolis multifidis. crispis anrantiis fulvis, scutellis concoloribus. In cortice.)

$\because 60$. I. polycarpa, subimbricata: Coliolis laciniatis flavescentibus, luteis, congestis, oblitteratis; scutellis undique tectis cleva-

$$
\text { I } 5
$$

tis. 
tis, margine concoloribus. Ehrh. crypt. exs. 136. (Lich. polycaipus.)

In cortice arbonum, fmucum. (Scutellarum discus etian in exemplaribus ex Anerica obten. tis saturatior rargine.)

6ı. I. laqueata, subinubricata lobatoorenata virescens; scutellis convexis rufe. scentibus. Jacq. Coll.5. 1.5. Jig.2. (Lich. leq̨ueatus $\Gamma \bar{v} u l f$. )

In montosis ad saxa. (Carinth. Wulf. Margo juniorum scutellarum actate deletur, co. lor virescens crustaceae frondis, subtus albidae, siccitate.)

*62. I. crassiformis, imbricata obtuse 10. bata virescens, subtus margineque nigricans.

In molltosis terrae inctrata. (Specinina Lich. crassi Hurds. ex Anglia, Italia, Gallia et regno Tumetano ad me delata, omuia in eo couveniuut, quod crustacea magis foliola sordide e fusco vi. rentia sint, subtus parum fusca albo margiue cincta, scutcllis copiosis albo-marginatis ex fu. sco foliis concoloribus, tecta. Odorem etiau spiraut debilem voteris Tabaci similem. No:tra planta Gott. et in vicinitate lecta, et pro Lich. crasso vendilata, colore magis virescente, etiam 
in siccitate, scutellarum et odoris illius defectu, margine nigricante constanter diflerc. Fïg. Flor. dan. $\iota .1005$, fuliornm colore convenit quidem, at L. crassiformis nunquim orbiculatim crescere solet. Illam potius cum Lich. chrysoleuco Smith, in Trans. lin. vol.2. t.4. Jig.2, depicto, et rostra Squamaria rubina Il. 7ich. r. 19. f. 1, quoad viva exeraplaria conjungere vellem.)

41. Psora '(Schorflechte) G. pl. 1686.

Crusta effigurata. Scutellae marginatie, couvexae.

* 1. P. squamata, crustacea imbricata: foliolis sinuatis rotundatis; scutellis nigris convexo - plauis. Dicks. fasc. 2. 20. Ej. hort. sicc. brit. 3. 25. (Lich. squamatus.)

In montosis, ad rupes, terram. (Iuterspersum observo Collema-ramulosum nigrum multi. fidum teres obtusum.)

* P. turbinata, crustacea imbricata : foliolis abbreviatis crenulatis; scutellis turbinatis rugulosis nigris.

Gum priori.

2. p. carnosa, crustacea imbricata: fon liolis confertissimis erectiusculis rotuudatis rufescenti carmosis margine albido cre14 nulato 
nulato farinoso; scutellis crassis crustae con. formibus.

In montosis, rupestribus. (Etiam ex Alpinis Norreg. Labco. An h. 1. Lich. Carnosus Dicks. fasc. 2. 4,6. $\int_{5}, 7$ ? Aiin ex Hercyn. montosis observo plantulam: foliolis depressis rimoso aul gnlosis; scutcllis planis rufis marginatis : margiue crusta concolore: P. gelidiformis, a Lieli: gclido Flor. dan. . 470. Jig.2, certe diversissi: เna.)

*. P. decipiens, crustacea frustulosa ag-i gregata lateritia; scutellis comvexis margi- Halibus uigris. $P l$. lich. t. 45. fig. $1=5$. Jacq. coll.5. t.5. fig.5. (Lich. elveloides LTulf.)

In montosis ad terram. (Eandem ex America et Africa habeo. Foliola juniora scuelliformia. Variat colore obscure vel dilute testacen chiam caruco, b. 1: r. gypsoptilla, crusta globulosa carnca; scutellis nigris. Schrad. spic. 89. (Lich. Eypsophilus.)

4. 1. testacea, crustacea frustulosa aggregata cinerea; scutellis convexis marginalibus lufis. $P l$. lich. 1.22. fig.5-6.

In rupibus montosis. (In humido cincrco. virescems.) 
-5. P. paradoxa, crustacea frustulosa ag. gregata conrexa umbrino rufo-fusca; scutellis pruinatis albido-marginatis, demum convexo - planis nigris. Ehrh, crypt. exs. 206. (Lich. paradoxus.)

Ad terram locis montosis. (Frondes funiores scutclliformes, mariuatac, dein convexae fru. stulosae, in humidu virescentes.)

* 6. P. vesicularis, polymorplia inflata al. bido-cinerea; scutellis plano-convexis nigricantibus. $\mathrm{Pl}$. lich, t.32. fig. 3. (Patel. Ifria vesicularis.) Flor. dan. t. 1064. f.x. (Lich. cạndidus.)

Ad terram locis montosis sacpe cum priori consimili. (Jam ab initio globosa substipitata pruinata.)

* 7. 1 . similaris, crustacea frustulosa ag. gregata convexa albida: scutellis concolo. ribus marerinatis.

In saxis montosis, in lignis rarins. Frondes globulosae, etiam ex carueo albidze.)

8. $\%$ ostreata, crustacea frustulosa aggregata, imbricata superne convesa margiuata: margine pulverulento.

$$
\tau 5
$$


In cortice Abiet. ligno. (Color virescenti griseus.)

* 9. P. candicla, cristacea tartarea conglo. merata candida sublobata: lobis dellexis; scutellis pruinatis. $P l$. lich, t.53. fig.2. (Patellaria candida.)

Ad rupes, in terra.

10. r. cpigeja, crustacea tartarea rugosa albida sublobata; scucellis marginatis conrexis nigris.

Ad terrum, inter MTuscos (H. vid. 1. Lich. candicans, crustaceus sublobatus albido - caudi: caus sculclis pulvinatis nigrescenti fuscis albo: marginatis. Dicks. fasc. ó. 1aZ.9. fig.5. Lich. melanocarpus Pers. in An. bot.7. 25.)

*11. P. Muscorum, cinstacea leproso-tar. carea ruguloso - lobata; scutellis inmersis concavis nigricantibus. $P$. lich. t.21. f. 1. (Patellaria Muscorum.)

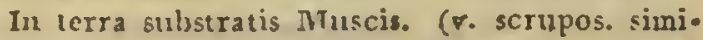
lis. H. 1. Lich. bryophilus E/rh. cropt. exs. 256.)

* 12. P. lentigera, crustacea tartarea lactea Lqbata: Lobis depressis rotundatis; scutellis Lenti. 
lentiformibus albo-marginatis. $P l$. lich. t. 48. fig. 1 .

In montosis mpestribus, ad terram.

15. 1'. cancseens, crustacea suborbicularis caudicans farinosa lobato - corrugata ; scutellis marginatis plano-convexis nigris. Dicks. fasc. 1. t.2. Jig.5. (Lich. canescens.)

In arborum cortice, palis, muris. (Raro cum scutellis; crusta plus minusve orbiculata in fo. liola obtnsa subirabricata, margine expansa.)

* 14. 1. albescens, crustacea suborbicularis albicans, lobato - conrugata; scutcllis pallidis.

In muris, saxis rupibus. (Scutellac depres. sae, crusta maryine concolore, denum oblitte. rato, disco dilute e cinereo carneo.)

* 15. P. citrina, crustacea leproso-tartarea sublobata lobis rugosis depressis; scu. tellis rufescentibus. $P l$. lich. t. 仍. fig. 2.

Ad terram, in montosis. (Eandesn e regno Tunetano a cel. Olof Linelen collectan habco. H. 1. Lich. fulgens Swartz, in nous. Act. upsal. 4. 246. Var. oolore dilutiore ex albo ct citrino mista crusta.) 
16. P. caclata, c:"rstacea tartarea lobata crispa: lobis depressis albo-marginatis ; scutellis rufescentibus.

In subalpinis, montosis. (Tyrol. Fuesscu Nixt. cerer - E cinerco flavescit subtus albida.)

*17. 1. Hypnorum, crustacea leprosa granuloso - foliolosa; scutellis rufescentibus, margine crusta concolore crenulato crispo.

Ad terram, MIuscis substratis. (Colore vi. rescenti cincreo, flavesculc: r. deaurata, orustacea Ifproso - gramuloso foliolosa nave. scens; scutellis rufescontibus margine crut şta concolore crispo ciuctis. Ad lentem foliolosa quasi crusta couspicienda. Etiam ex. Nor. vegiae alpinis habco. Scutellis majoribus spar. sis elcvatis margine crispo s. gramulato cinctis. a sequenti diǵnosimur. H. vid. 1. Lich. Hypuoxum Flor. clan. (.956.)

* 8. P. pezizoides, leprosa graunloso - foliolosa subcinerea; scutellis depressis fu. Bco - rufis margine cremulatis.

Ad terram, Muscis substratis. (Occurrit Ter. rae vel lapidibns innasccus confertissimis scutellis, crusta ald lentem obscurins folioloso graune Lose; r. mebulosa. Rl. lich. C. 40. fig. 1.) 
* 19. P. brunnea, leprosa granuloso - foliolosa brumnea; scutellis depressis concolo. ribus.

Ad saxa, in terra. (स. forsan 1: Lich. brun. neus Surarlz, multiflorus $1 \mathrm{hr} /$. $^{\circ}$ crypt. exs. 253 . Scutcllis in terra majoribus, in saxo adpsessis a crusta vix distingnendis.)

* 2b.' 'p.'microphylla, cíustacea granulosofoliolosa cineren; scutellis convexis rufe: scentibus.

Tn cortice arborum, ad terram. (H. 1. Lich. microphyllus Scturad. spicil. 1.1. fig.4. Ad lentem foliolis gramulosis rimutis cinereis ernsta composita; aetate nigrescentibus.

* 21. 3. grisea, crustácéa géranuloso folio loṣa griseo - cinerea; scutcllis nigris a cru. sta marginatis: margine crenulato; demum convexis.

In arenosis, turfosis:- (Per lentem granula crustae in foliola coagmicutata, munc dilitiora nunc oluscurioza colore.)

*22. 1. variabilis, crustacea leproso-tarı. rea margine sublobata diluse nmbrina, albido-cinerea; scutellis confertis fusco : doesiis albido - marginatis, 
In saxis, lapidibus. (H. 1. Lich. variabilis rers. in An. boc. 7. 25.)

*25. $\boldsymbol{x}$. dendritica, crustacea cinereo-nigrescens dendritica; scutellis conrexis concoloribus. Pl. lich. t. 19. fig. 4. (Verru. caria dendritica.)

Crystallo quarzosae, etiam calcario lapidi in. nascitur. (In montosis Hercyn., saepe in con*) sorito $\mathrm{V}$. coutig.)

24. T. upsaliensis, crustacea tartarea frut ticuliformis depressa; scutellis pallidis," margine crusta concolne candido. Pl. lich. t.21. Jig.2. (Patellaria upsaliensis.)

Locis montosis, sterilibus. (E variis Germ. locis accepi Muscis rel graminibus instratam eaque incrustantem, mude facies fruticulowa rol snbuliformis, quod ctiam in L. tartareo sed ra., rius ficri solet. Scutcllae Perellac similes. Aliam alo $T h r / h a r t o$ Upsaliac, etiam in Nordlandia ab $O$. Zinden, lectam accepi plantam, lignis, corticibus, sunascentem, quoad scutcllas Upsalieusi simi. lem, hiuc inde lividas, crusta verrucosa: $P$. alabastrina. Scutellarum colore differt: $\dot{\mathbf{p}}$. fri. gida, crustacea fruticuliformis candida; scutel: lis planis ochraceo - carneis albo - marginatis. Dicks. fasc. crypt. 2. 29. Ej. coll. of dried $p h$. 


\section{(IICHenes.)}

1. 49. Isidium frigidum. Achar. in $K$. Tet. Acad. 11. Handl. 15. t.6. fig. 2.)

25. P. lactea, crustacea tartarca orbicularis candida, margine sublobata centro ri. moso-radiosa. Enum. Lich. tab. 4. fig. 6. Jacq. coll.5. t.4. (Lich. lactens Ifulf.) .

- In rupibus, saxis. (Crusta in globulos faxinaceos. efforescit, xarins scutellifera: scutellis atris.)

-26. 1. Bracteaca, crustacea orbicularis faruosa xugulosa margine sublobata citrina.

In montosis rupestribus. (Absqne scutellis.).

42. VERRUCARIA (IVarzenflechte) G. pl. 1668.

Crusta aphylla: leprosa, tartarea. Scus rellae marginatae, immarginatae.

\section{Scutellis decoloratis.}

1. V. Perella; tartarea verrucoso albida; stutellis concoloribus obtusis marginatis. Pl. lich. t. 12. Jig.5. (Patellaria Perella.)

In rupibus, muris. (Crusta ex cinereo ctiana rufescit.)

Fi 
* v. tumidula, crustacea alhida; scutellis concolor bus margine tumidulis.

In cortice. (H. 1. Lich. tumidulus Pers. in An. Zot. 11. 18.)

* 2. v. faginea, crustacea; veruucoso, candida scutellis concoloribus.

In cortice arborum, saxis. (Occurrit:

* v. orbiculata, margine zonata, disco scutellifero. Enum. Lich. t.7. Jig. 2.

In cortice arborum.

* V. discoilea, crustacea Effusa; scutellis Sarinaceis, nudis. Enuit. Lich. l. c. fig.5. In cortice.

* ${ }^{*}$ : uberculosa, crustacea effusa; scntellis verrucoso-farinaceis, confuentibus. Enum. Lich. t.2. fig. 4 .

In arboribris, saxis.)

* v. modulosa, crustacea nodulosa candida; scutellis excavatis margine concoloribus.

In cortice, super IITuscos. (Occurrit crusta nodulosa: nodulis apice ruyulosis, sculellisque pallide e carnco rufescentibus V.rugulosa. H. is qquoad saltem figuram Lich.' albo Aaresceus IFulf, in Jacq. Coll. 3. t.5. Jig. 1.) 
5. จ. farinosa, pulverulenta candida; scutellis pallidis maxgine concoloribus.

In cortice. (H. vid. 1. Lepra farinosa Enum. Lich. t. 1. Jig. 1. Rarissime cum scutellis, sine his crusta farinosa similaris lactea.)

* 4. V. umbilicata; leprosa cinerea; scutellis candidis minutis umbilicatis.

In rupibus, saxis. (Gott. Fleyd.)

* F. albella, crustacea lactea ; scutellis con. coloribus.

'In cortice arborum. (Ad lentem discris palli. dissime carnens. H. 1. Lich. albellus Pers. in An. Got. 11. 18.)

*5. v. pallida, crustacea albido-cinerea; scutellis pallidis albido-marginatis.

In cortice arbornm. (Discus e cinereo palli. de carneus. V. angulosa, scutellis confertis de. mum angulosis convexis. Jacq. Coll. 3. C. 5. fig. z. a. Lich. pallescens Wulf. Schreb. spic. 1132, 1137. Lich. pallidus, angulosus. Occurric scutellis crassioribus margine tumidis crusta ae. quabili albido cinerea, qualem ex America ha. beo: V. cancriformis. Etiam crusta margine 20. hata sed rarissime.) 
*6. v. impolita, crustacea exasperata alhido-cinerea; scutellis difformilus pallidis. Eluh. crypt. exs. 274. (Lich. impolitus.) "In cortice rimoso, saxis. (Scutcllae quasi. pruinatae e cinereo caesiae, intus leniter rufe. sccutes. H.1. Lich. pruinatus Pers. in An. bol. 11. 19. - Occurrit scutellis in crustam impres. sis: crusta in rupibus c cinereo rufricente; scutellis emersis, etiam levissime uigricautibus.)

* 7. v. glarcoma, crustacea, tartarea albido - cinerea; scutellis velatis pallidis, crusta concoloribus, cacsiis, demmm nigricantibus. $P l . l i c 7 . t .52,55$.

In eaxosis rupestribue. (H. 1. Lich. sordidns Pers. in Aı. bot. 7. 26. Iich, allido-cacsius Schrad. quicil. 37. Iich. varians Davies, in Trunsact. Tin.8. 1. 28. Sig. 5.)

\section{Scutellis rufescentibus vel lateis.}

8. v. detrita, crustacea altido-cinerea; scutellis confertis difformibus deritis, a crusta marginatis.

.In corriçe a rborum. (Ab initio scutellae pal. lidae, a crusta vix distinguendae, demum emer. are nufesceutes subfuscae, marginatae.) 
* 9. v. tartarea, tartaren globulosa albida; scutellis planis albo - marginatis ochraceo:xufescentibus. Flor. dan, t.712. Jacq. coll. 4. t.8: Sig.2. (Lich. tartarcus, Vitulf.)

In rupibus, cortice. (H. 1. Lich. androgyntrs Enumu. Iich. 1.7. Jig. 3. Crusta glelulosa; 'fa. rinosa, in recenti e cincreo virescens; muscis instrata aegre a $P$. frigida distingnenda.)

10. v. granulosa, leprosa incana; scutel. lis convexis. rufescentihns. Pl. lich. t. 30, fig. 5 .

Ad terram, super muscos. (Scutcllae alı in: tio planiusculac, mox vero convexac. Occurrif scrutellis pallide c cameo rufcscentibus i cl fusco rufis, ab initio globosis vel convexis; crusta etiam virescenti incana. Aliam e Norlandia ace. cepi plantulam scuicllis globosis camosis fursco. rufescrutibus, muscis ut prior instratam; not mine Lich. Leuccopfiaei Flor. dan. togjj. fig. 2.)

* 11. v. rufescens, leprosa cincrea, nigri. cans; scutellis contrexis immarginatis rufis: Pl. licl.. t. $17 \cdot$ fig. 1.

In rupilus, saxis. (Occurrit: crusta obscrs. ra, scutellis dilutioribus depressis, vel sordide Infescentibus, vel crusta olivacco-flavescentr. 
H. vid. 1. q110ad fig. Lich. viridi-Jlavescens Hulf. in Jacq. Coll.3. t. 2. fig. 1.

$\therefore$ 12. v. effusa, lcprosa cinerea dilute aeluginosa; scutellis rufescentibus, margine crusta concolore cinctis.

In ligno; praescrim Salicum, quas interne saepe longe latcque inrestit. (Gott. Pers. Lich. effusus. Lich. salignus Sc7hrad. spicil. 94. Seu. tellae occurrunt pallide e carneo rufescentes, cil fusco- nufae confertissimac r. pezizoides si. miles, margine tenui flavescenti-viresceute cin'ctae ; disco demum convexo.)

13. v. conglcinerata, leprosa cinereo-viiescens; scritellis convexis globosis dilute ex carneo et Havo rufescentibus, in acerrulos congestis.

$I_{11}$ ericetis ad terram, in cortice. (Gott. Pers, Lich. conglomeratus. Heyd. - H. vid. 1. Lich. sphaeroides, leprosus cinereo - virescens, tuber. culis globosis incarmatis. Dicks. fasc. 2. t. :: fig. 2. Margo in junioribus scutellis ad lentem hinc inde conspiciendus disco concolor.)

* 14. v. rubella, leprosa albido-Alavescenti cinerea; scutellis rufescentibus, margine pallidiore cinctis. Ehrh. crypt. exs. 196 . (Licl:, rubellus.) 
In cortice. (Occurrit: crusta leprosa-navescente scutellis demum conyexis rufescautibus margine disco concolore V. verralis. Lich. verualis Euum, Lich. t.5. fig. 2. - Scutellis turbinatis. Color marginis in sicco pallide.çarneus. Scutellis dilutioribus margine concolo. ze, crusta fere nulla Lich. lucteolus Sc7trad. spi. cil.85. - V. fusco-rubelln, scutellis elevatis fusco - rubellis, disco plano, znargine conco. lore.)

15. $\nabla$. coronata, crusta ciuerea leproso.granulosa; scutellis rufescentibus depressis a crusta granuloso margine cinctis, disco convexo.

In rupibus, ad terram. (Duplicem varictatem coram liabeo, mam e Gallia rupibus inuascen. teun crusta cinerea distincte gramulona; scutel. lis e carncovel ferrugineo rufescentibus; alteram in terra, Gottingae lectam, crusca c cinc. reo nigricante ad lentcm tantum.grannlosa scu. tellis minoribus, e fusco rufesccutibus, in siccitate nigricantibus.).

16. v. rubra, crustacea albida; scutellis concay is carnco-rufis, albido-crenatis. $P l$. lich. t. 1\%. fig.2. (Patellaria rubra.) Enum. Tich. t.5. fig.2. (Lich. pallidus.) 


\section{I76 cRYPTOGAMA SCUTELIATA}

- In cortice àrborum, liguo, muris. (H. L. Lich. ulmi Sivare. in act. ups. 4. 2/77. Lich. prui. uosus frumb. frit. g. Color, disci ex pallide carteo sensin in carnco. rufesceutem vel fuscum (rausit.)

* 17. v. rosella, leprosa crustacea cinereoalbida; scutellis convexo-planis incarnato-rufis, margine pallidioribus.

In curtice arborum. ( $\mathrm{H}$. 1. Lich, rosellus Ters. it A 12 . bot. 7.25 . Lich. albo-incaruatus, leproso-crustaceus, tener, globulosus, allus, mberculis lentiformilus iucarnatis $W_{\text {ulf. in }}$ Jacq. Coll.3. t.2. fig. 3. Partes fructificationis per microspium compositum spectatae, aflinita. tein ostendunt c. Lich. Baconyces.)

18. v. cupularis, leprosa; scutcllis excavatis carneo-rufis, margine tumido extcrius dehiscente.

Iu rupibus, saxis. (Crusta tcusissima occur. rit colore nigricante, cincreo, miniato. Sclin tellae juniores ore contractae, margine cxpanso dehisceusc, per lentem radioso. II. I. Lich. cu. prilceris Jihth. Beitr.4. 45. Hediv. stin). 2. 1.20. - Cichi. fusco - rubers IVulf. in Jacq. Coll. 万̃. r. . fig. .. Lich, sonanoreus Lium. Lich, 1.6. t:s.: 4.0 
* 9. v. decolorairs, leproso-granulosa ci-neren; scutellis. planis marginatis rufescenti carneis, nigricantibus.' $N$. lich. t.39. fig. 2. (Patellaria decolorans.)

C In tcrra' turfosa. (Hercyn. Pers. - Aliam in montc Rammelsberg ab Ehihorto collectain speciem habco V. incolorutann: crusta leprosa cincrea; scutellis planis marginatis pallidis mimuis, margine crusta concolorc; jutiori graniformi similibus. In terra.)

* 20. v. clausa, leprosa; scutellis immer. sis clausis, demum expansis concavis crustae foveolis inmersis albis. Enum. Lich. 48. ' (Lich. clausus.) Trans. lin. 10: t. f. fig. 1. (Licb. exanthematicus Sinith.)

un saxis, rupibus calcareis.) (Scutellarum discus pallidissime rufesccus, elaberss.)

*21. v. ferruginea, leproso - crustacea albido-cinerea; scutellis rufescenti - ferrugineis margine concolore. Pl. lich. t. 12. f.x. (Patellaria cinereo-fusca) i. 25. fig. 1. (Patellaria ferruginea.)

In corice arbornm. (H.'1. Lich. ferruginess Iluds. quoad spccimina ex Aliglia acceptra Crilstam, sed rarius, etiam lutescusem olseryayi.) 
*22. V. arenaria, crustacea leprosa cine. rea albido-caesia; scutellis rufescenti-ferrugineis margine dilutiore.

Ad saxa arenaria. (II. 1. Lich. arenarius, crusta leprosa pulvernlenta caesia; scutellis cro. ceo - rubris Pers. in Al1. bot. 7. 27.)

* V. caesio - rufa, crustacea albido-caesia; scuteliis rufescenti ferrugineis, margine lle. xuoso albido.

$\Lambda d$ saxa arewaria. (H. 1. Lich. craesio rufus, crusta tenuissima granulosa cacsia, scutellis sparsis rufis Schrod. spic. 80 . Scutellae aetate discolores.)

* 23. v. aurantiaca, leprosa subcinerea scutellis aurantiis rufescentibus flavo mar: ginatis. Enum. Lich. 45.

In cortice arborum, saxis. (H. 1. quoad specimina ex Anglia accepta: Lich. flavo . rube. acens IXuds. Praeter crustam luic valde similis : )

*24. v. oblitterata, leproso-crustacea gllebulosa sparsa flavescens; scutellis confertis plano. convexis rufescenti aurantiis.

In nupibus, saxis. (Crusta a scutellis angir. Iatis oblitterata. H. 1. Lich. oblitceratus, rupie. stris, 
stris, crusta globulosa effusa flava, margine subfoliasceute, scuteilis incdio congestis auran. tiis Iers.in An. bot.11. 15. V. holocanna, leprosa aurantia; scutellis confertis rufescęuti anrantiis. Ehrh. crypt. exs. 284. Lich. 7iolo. carpus. In sepimentis, ligno. Aliam ex subal. pinis Norvegiac nomiue Lich. Jungermannize Flor. dan. 1053. fig. 1, accepi : crusta leprosa cincrea; scutellis rufescenti - aurantiis sparsis planis demum convexis, inargine concolore. Iu Muscis, Jungermannia alpiua.)

25. v. viridi - rufa, leproso - crustacea Aavo- - virescens; scutellis marginatis ufusceriti - aurantiis. Jacq. coll.2. r. 15. Jig.4. b. (Licl. Alavo-virescens $W_{u l f}$ )

In rtpilutss, saxis.

'26. v. cerina, leproso - crustacea subcinerea'; scutellis cerinis albido-marginatis. Il. lich. t. 33. fig. 1. (Patellaria ceriuta.).

In cortice, liguo. (Occurrit pro ratione sub. jectae crustac, margiue plus minusve cinereo. Crusta fere nulla; scutellis sordide cerinis cine. reo-marginatis: v. gilva. In ligno. Scutellis rufescenti - ceriuis albido - marginatis demum convexis: $\dot{v}$. aureo-cerina. Etiam occurrit scutellis minutissimis flavis albido vel tamen palli. dius disco marginatís Vitelliuo similis.)

M 5

* $v$ 
180 CRINTOCAMIA SCUTELLATA

* V. ravila, scutellí coufertis umbrino fusco luteis, crusu ciucra margine cor. colore.

lu cortice.

* v. stillicidiurum, leprosa víridi - cinerascens; scutellis cerinis, viridantibus cinereo - marginatis. Flor. dan. t. 1063. fig. 2. (Lich. stillicidiorum.)

Muscis inspersa ad terram. (Gottingac lecta scutellis ysiltacinis minutis cinerco - marginatis hinc inde cerinis, demum lividis, plumbeiv; Norvegiae, scutcllis parun viridantibus magis cerinis, cetcrum planc similis.)

\section{Scutellis fuscis vel nigricantibus.}

- 27. v. flavo-fusca, crustacen obscure $\AA_{2}$ vescens; scutellis sparsis convexis rufesceatti-fuscis.

In saxis arenosis. (Epicosp. Hildes. Schrad. gic. 8b̈. Lich. Javo-(iuscus.)

28. T. subfusca, crustacea cinereo - albicans; scutellis rufescenti fuscis, margino crusta concolore. Enum. Lich. t.5. fig.5. In cortice, saxis, muris. (Occuxrit: 
* V: argenteo - fusca, crusta albida; scutellis saturate rufescenti fuscis, margine albido cirictis.

In cortice. In saxis, muris; crusta subcinerea saepe orbiculatim expansa; scutellae confer. tac saturate ex nigro fuscac.

* v. pallide-fuscu, crusta albida; scutellis pallide rufescenti fuscis, albido - margina. tis.

In cortice arborum, Piu, sepimentis. Scu. tellae pallidae, discolores, augulosac.

* r. unbrino - fusca, crnsta cinerea: scutellis umbrino-fuscis, cincreo - nurginatis.

In cortice. (E, 1. Lich. umbrisus Ehrh. cropt. cxs. 2(jo)

* r. fusca, crustacea cincrea; scutellis confertis depressis nigro - fuscis albido - ınaxgintis.

In rupibus, saxis. (Copia scutellarum crustam obfuscante, ex meris scutellis nigris vide. sur constare.)

29. v. fusco-atra, clustacea cinereo-fusca rimosa; scutellis nigris depressis contsigntis. Jacq. coll. 2. t.14. Jin. 5. (Jich.

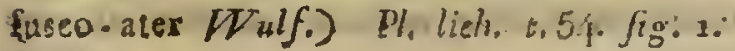


In saxis rupestribus. (Occurrit crusta e gri. seo dilute fusca.)

* vincreo-aira, crustacea cinereo-griseoaltida rimosa frustulosa; scutellis nigris depressis stubcontiguis. Jacq. coll. 2. t. 14. fig. 1. (Lich. atro-albus IT ulf.)

In rupestribus, saxosis. (Crusta dilute e griseo in ciucreo carneum hinc inde tendit, par. ticulis rotundatis.)

* 5o. v. badia, crustacea olivaceo-cinerea rimosa; scuellis plano-convexis badiis, margine crusta concolore. Pl. lich. t.5x. fig. 2.

In montosis rupestribus (Hercyzı. E/2r/2. Pers. Lich. badius it An. bot. 7. 87.)

* V. griseo-atra, crustacea griseo - cinerca rimosa; scurellis nigris depressis contiguis.

In rupestribus saxosis. (Scucllac ad lentem marginatae crustac impositac, in ultima et pen. ultima crustan aequantes.)

* 51. v. atro - allia, crustacea rimosa fitu. stulosa atro alboque mixta.

In montosis, saxis. (Hercyn. - Eanden in Galliae et succiae montosis collectam observo 
albidis particulis crustae nigro conspersis pui. vere.)

32. v. coracina, crustacea nigra rimosa; scutellis confertis impressis atris.

In saxis rupestribus. (Nudo oculo nigra, ri. mosa; ad leutem subcinerea, scutellis intra par. ticulas crustae immersis. Occurrit crusta inae. quali frustulosa, scutellis atris cincreo-margi. natis, e crusta emergentibus. H. l. Lich. atro cinereus, crustaceus ater, scutellis confertissi. mis planis nigris cinereo - marginatis Dicks. fasc. 3. $t \cdot 9 \cdot(\mathrm{gig} \cdot 2$.

*33. v. atra, crustacea cinereo - albicans : scutellis atris albo-marginatis. Enum. licli. t. 4. fig.6. (Lich. ater.)

In rupibus, cortice. (Crusta aequabilis, ri. mosa, grumosa. H. l. Lich. cinerens Wrulf. is Jacq. Coll. 2. t. 14. fig.5. b. Lich. tephromelas Ehth. crypt. exs.313. v. atrata, crustacea cinerco.albicans; scureilis minutis atris albo. marginatis. In cortice.)

34. v. ocellata, crustacea tessellato-verrucosa cinerea, scutellis nigris ocellata. $\mathrm{Pl}$. lich. t. 20. flg. 2.

In montosis saxosis. (v. ocellulata, crustacea rimosa tesselata cinerea; scutellis minntis simis 
simis occllata. In tectorum, tegulis, rupibns, saxi..)

* 55. v. confucns, crusta acquabili fumo. so - cincrea; scutellis cunvexis atris conflucutubus. Pl. lich. t. 10. f:g. 1.

In rupiluus saxis (Ifercyn. It"ol. spic. t. 1. fig. 2. Lich. confuens. Occurrit scutellis ad. lentem mglosis.)

* 56. v. conligua, crustacca rimosa subcinerea; scutellis nigris depressis contiguis.

- In saxìs. (Sculcllac ad lentem nigro-margi. suatac. Occurrit crusta albido-cinerca, scutel. lis convexis, vel crustac insidentibus; crustae areolis cicatricosis : v. (icalricos $\pi$.)

* V. petrrea, crustacea sulucincrea; scutel. lis nigris depressis concentricis. Jacq. coll. 5 . t.6. fig.2. (Licls, petraeus TVulf.) $\mathrm{Pl}$. lich. 1.5\%.

In saxis, rupibus. (Crusta cliam albida.)

* vantherina, crustacea allida; scutellis depressis nigris margine concolore cinclis nigris comtignis excentricis.

In saxis, rupibus. (Al) ullima differt scutel. lis cxcentricis a penultima crusta et acutellanm parvitate.) 


\section{(IICHENES.)}

*. tigrina, crustacea aequabilis lacteocandida; scruellis nigris depressis a crusta marumatus demum convexis, contiguis excentricis.

In mpibus saxis. (Scutcllarum magnitudinc sim ac forma, facile a sequenti distingnitur.)

*37. v. calcaria, cristacea effusa lactea; scurellis convexis atris a crusta marginatis.

In saxo, lapide calcario. (Occurrit crusta fa: rinosa cretacea: Lich. cretacens $E / h r /$. Beier: 7. 12.)

38. v. tessellata, crustacea cincreo-Jacteá aequibilis rimnso - tessellata; scutellis im. pressis a crusta marginatis. Flor, dan..t. 468. fig. 2.

1 rupibus, saxis, Japidibus, praesertion cal. cariis. (Areolac ab initio punctatac disco sen: sim expanso scutelliformi concavo, cincrascen: tc, nigricante, in areolas tessellatas guasi immer: so, actate simul cum arcolis encrgente. ' $H$. v́: 1. Jich. punctatus Dicks. Jasc. 5. 25. Lich: ri mosus Tielz. scand. 2.1529 . exclusa fig. 5. to cit. Fl. dan.)

*39. v. multipunctu ortustacen cinereo- 12 atea requabilis rimoso-tessellata; scutellis 
intra arcolam pluribus impressis contiguis, demum prorumpentibus marginatis.

In montosis rupestribus. (Hercyn. $\rightarrow$ )

* 40. v. scruposa, crustacea verucoso - rimosa cinerea; sciutellis immersis concavis nigricantibus. Pl. lich. t.11. fig. 2: (Patel. laria scruposa.)

In rupibus, saxis, muris, palis vetustis, muscis. (Variabilis Licherl occurrit crusta semper ạspcriore quam antecedens species, albido ci. nerea tartarea, scutellis lividis; cinereo. cace sia, dilute ferruginea, vel eleganter pallide sul. phurca, scutellis plus minusve nigricantibus, margine crenulato vel integro aperto, contra. cto. H. 1. Lich. pertusus I'ulf, in Jacq. Coll. 2. 1.15 . fig.5, ut specimina viva rev, auctoris evincunt, a Licl. pertuso aliorum auctornm, quem ctiam rupes inlabitantem possideo ct quarn Iongissime a Lichenum ordinc diversum puto, distinguendus. - Lich. ocellatus Gmel. Syst. Nat. 1361 . Lich. impressus Swartz. in nov. Act. zups.245. - Similema crusta albicante scutellis impressis lividis in Regino tunetano lectam co. ram liabeo.)

*41. V. contorta, crustacea albida; scutellis immersis nigris margine spiraliter coutortis, Pl, lich. t.22, $\sqrt{\mathrm{gg}} .1 \cdot 4$. 
Ad rupes, saxa, lapides. (Occurrit scutellis simplici duplicive margine contortis; confluentibuls vel distinctis. Crusta parașitica niıgra.).

* 42. v. immersa, cinereo - albida, lactea ; $8 c u t e l l i s$ immersis nigris. $P l$. lich. t. 12. $\operatorname{sig} \cdot 2 \cdot 4$.

In rupibus, saxis. ( $V$. calcivora, scutellis migris planis in calcem immersis. Ehrh. crypt, exs. 24\%. Lich. colcivorus. V. inmersa scutellis ad lentem marginatis, frequenter in consortio vegetabilis alins ordinis: Lich. antiquitatis Enum. Lich. t.3. Jig. 5, et V. rupestris Schrad. joic. soy. occurrit.)

43. v. purpurascens, roseo - purpurea; scutellis immersis nigris. $P l$. lich, t, $15^{\circ}$ fig. 1.

In rupibus calcariis. (Carinth. Wulf. Lich. marmoreus. In Jacq. Coll. 2. t. 13. fig. 3.)

* 44. v. silacea, crustacea silacea; scutellis convexis nigris margine introhexis. $l l$. lich. t. 19. fig.2. (Patellaria silacea.)

In montocis rupestribus. (Hercyn. - Margo scutcllarum introllexus, in papillam sacpe ele. vatus.) 
* V.Octeri, crustacen silaced; scutcllis de. pressis nigris, difformibus, contiguis. Flor. dan. t. 470. fig. 1.

lisdem cum prịori locis. (H. vid. 1. Tich. Oederi, leproso - crustaceus rimosus ferugi. neo - rubens, mberculis congesıis innanis diffor. nibus atris submarginatis Dishs. frasc. 2. 11. Lich. Koenigii frumb. frib.47. - Crusı hinc inde cinerea colore prioris, sculcllac majores v. conliguae similes.)

* 45. v. grumosa, crustacea grumosa caerulescenti cinerea; scutellis sparsis depressis niga is albido - marginatis crenulatis.

Ad saxa arenaria. (Gott. I'ers. Lich. cacru1escens, in An. bot.22. 25. Lich. grumosus 7 . c. 14.34.$)$

46. v. ccerulescens, leprosa crustacea ex albido caerulescens; scutellis concoloribus margine pallidiore cinctis, demm nigricantibus, Hag. Lich. t.1. fig.5. (Lich. caerulescens.) Enum. Lich. 45.

In rupibus, arbornm cortice. 
47. $\nabla$. albo-caerulescens, cristacea aequabilis albicans; scutellis complanatis pruinatis allo-caerulescentilus nigro - nıarginatis. Fl. lich. t. 14. fig.2. (Patellaria albo - caernlescens.)

In saxis, lapidibus. (Carinth. Wulf. Lich. albo-caerulescens, in Jacq. Coll.2. t. 15. Jig. I. In rupibus Friberg. IIurnb. Vermcaria annula. ta Flor. frib. 45. - Aetate et iuns nigrescumt scutellae. Crusta hinc inde etiam lineolis se. pta. Excepto loco natali et rufescente intus di. sco huic similis:)

* v. cyanescens, leprosa albicans; scutellis, complanatis pruinatis rufo - caerulescentibus nigro-marginatis. Pers, in An. bot.21. 17. (Jich. cyanescens.)

In cortice arborum. (Etiam linea nigra septa crusta.)

* 48. v. dispersa, crusta leprosa subnulla; scutellis sparsis umbrinis margine subtusque albidis.

Ad saxa calcaria, lapides. (H. 1. Lich. disspersus, crusta leprosa subnulla; scutellis dis. N 2 opersis 
spersis ninutis subfuscis caesiì nigrisque Pers. in An. bot. 7. 27.)

* 49. v. fumosa, crustacea rimosa fuliginosd; scutellis immersis mgris marginatis disco convexo prominulo. $\mathrm{Pl}$. lich. t. 49. fig. 2 .

In saxis arenosis.

*50. v. fasciculata, crustacea rimosa fru. stulosa fusco - lurida; scutellis minutis confertis nigris, expansis margimatis.

In rupestribus suonlosis (Hercyn. -)

* 51. v. uligimosa, leprosa ferrugineo-fusca: scutellis minutis depressis nigris pla. $210 \ldots$ convexis.

In uliginosis turfosis (Hercyn. Schrad. spic. 88. Lich, uliginosies, crusta gramulosa e fernugineo - fusca, veruucis subrotundis nigrescentibus. Scutellae minntac ubsque lente vix a cru. sta distinguenda. Occurrit: crusta leprosa distincle gramulosa cincreo.fermginea, scutellis uigris convexis contiguis coufluentibus V. hus mosimilis.) 
52. v. humosa, leprosa nigricans; scutellis convexis confertis fusco-nigris. Elwh. erypt. exs. 155 . (Lich. humosus.)

In terra. (Hannover. - Ehrh. Aliam ob. servo; v. viridem, in terra: crnsta granulo. sa by ssacea, tuberculis scutelliformibus fusco nigris. - Inter Fhascum scrratum et subnla. tum, ratione habita partium fructus (semina enim ope microscopii compositi intra thecas li. weras coordinata apparent) ad alintn ordinem amandanda.)

53. v. Muscorum, Jeprosa incana cinerea; scutellis planis, convexis nigris. $F l$. dan. t. 1003. fig. 1. Jacq. Coll. 4. t.7. f. s. (Lich. Muscorum INulf.)

In muscorum stratis. (Occurrit : scutellis conatanter convexis immarginatis, vel ab initio planis marginatis demum convexis, a sequenti forsan loco natali tautum diversa; scutellis dilute ex fusco nigris crusta vix disccruenda, leucophaco vel graniformi similis.) 
54. v. runctata, ciasta leprosa subcinerea; scutellis nigris planis, convexis.

In cortice, ligno, saxis. (Etiam acrustacea. Occurrit : )

* v. limitata, crusta subcinerea; scutellis nigris linea repanda septis. Enum. Lich. t.5. fig. 5. (Lich. sanguinarius.)

In cortice, saxis. (Eniam acrustacea.)

* v. guttata, crusta leprosa subcincrea ; scutellis nigris planis, convexis guttatis.

In saxis. (Etiam acrustacea.)

* V. atro - sanguinea, crusta inconspicua; scutellis plunis atro - sanguineis, nigro marginatis. Enum. Iich. 20. D.

In saxis, lapjdibus. (Ferc acrustacca. Scutcllae minutac, numerosac adpressac, humecta. tae atro vel fusco. sumguincac.)

* v. olivacer, crusta leprosa c cinereo di. Inte olivaceo - virescens; scutellis nigris.

In cortice, ligno; in saxis (sordide olivacea. scutellac hinc inde ex nigro fuscescuut.) 
* $v$. puncriformis, crusta leprosa albido. cinerea, scutellis minutissimis nigris punctiformibus.

In cortice rimoso Abicl, saxis. (Scutellae ad lentem marginatac, crustae inuumcrat insiden. (e6.)

55. v. abietina, crusta leprosa sparsa albida; scutellis nigris contexis albo - marginatis, demum hemisphaericis. Ehrh. crypt. exs. ${ }^{6} 66$. (Licl. abictinus.)

In cortice rimoso Abiet. (Sculline V. calcareac similes, hinc inde etiam discolores basi subfusca vel dilutiore, centro nigro, disco ela. pso, cxcavaliones undas obscrvo. Vel ad hanc vel ad sequeutem spectat: Lich. pilularis, cru. staceus cinereo - albidus tuberculis pilulae formibus nigris Davies, in trans. lin. 2. t. 28. (ig. 1i)

* v. pilularis, crusta albida; scutellis nigris convexis liemisphaericis imnarginatis.

In cortice Abiet. (Scutellac juniores ad len. tem margine concolorc instructae.)

56. v. allo-atra, crustacea albida; scu. tellis nigris pruinatis. $M$. lich. t. 15: f.2.

$\mathrm{N}_{4}$

In 
Ill cortice, saxis. (Occurrit scutcllis ab in. nio pruinatis, demum convexis undis; scutellis complanatis difformibus minutis pruinatis crusta amylacea: V. antlacere, Jhrhe crypt. exs. 503. Lich. amslaceus. I'crs. in. an. Zot.11.18. Sphacria byssacea theig. Obs. 42.

* 57. V. escharoides, crusta crassa leproso granulosa cervina colıerens, inaequabilis; scutellis nigricantibus convexis tubinatis. Elırl. crypt. exs.515. (Lich. escharoid.)

In turfosis. (Crusta crassa scmipollicari, ill. terue nigricante, superficic hinc inde e cincreo ferrngine2. - IIercyn. Gott. -)

*53. v. capircllata, crustacea albido-cinerea rimoso - verrucosa cicatricosa; scutellis minutis migris sessilibus stipitatisse. Enum. Lich. 4. 4. fig. 3 . (Iich. cincrens.)

In saxis, crista crassiote; in cortice arbo. rum; etiam orbiculatin expansa.

-59. v. sanguinaria. crustacea cinerco. virescens, albicans; scntellis convexis ni. gris intus sngunineis. 1l. lich. t.41. fig. 1.

In arborum cortice. (Hercyn. Gott. - Par. trum fructus ad microspinm compositnm exami. nati, 


\section{(IICIINTS.)}

nati, ratioue habita, vix inser Licluenum ordi. mem amplins militabit.)

\section{Crusia flazescente.}

*6o. $\nabla$. quernea, leproso-farinosa e viridi cinerco-Intescens; scutellis sparsis nigris. Dicks. Jasc. 1. t. 2, f.g.5. (Lich. querneus.)

In quercuum truncis putridis. (Innascitur sae. pe crustae Opegrapha pulicaris, caute a routellis orbiculatis convexinsculis confuentibus vel distinctis, distingnerida.)

* 61. v. lutescers, leproso-farinosa lutescens verrucusa; scucllis margine concoloribus, $M$. lich. t. 25 . Jig. 1-2. (Lepra lutescens.)

In cortice rimoso arbornm, intercurrente sae. pe V. faginea. (Scutcllae rarissimac ab initio marginatae, demun disco convexo margine fle. xuoso, oblitterato. Discus e carneo et havo di. lutissime rufercit. Opographa ut in priori sac. pe crustae insidens.)

* v. inaculiformis, leproso - fariuosa late. scens maculiformis; scutellis margine corrcoloribus. 
In cortice Lctular, Alictis. (Maculas efformat semipolicares, pollicares lutcsceutes; scutcllae plerumquc confertac, colore ul in priori, hinc inde paulo ouscurioris coloris. Eandem ex Nor. vejid habeo.)

* 6i. v. varia, crusta navo-virescens scutellis oblitterata variis, umbrino - fuscis, margine crusta concolore cinctis. $\mathrm{Pl}$. licle. t.23. fig. 4. (Patellaria varia.)

Ad ligua sicca, asscres. (H. L. Lich. pallesccus schronte, bavar. 1504, quoad specinina viva Auctoris.)

* 63. v. polytropa, Havo-vireus scutellis undique conspersa plano - convexis e dilu. ie caruco Ravescentibus. Ehrh. crypt. exs. 294 .

In saxis monlosis. (H. 1. Lich. atro - vircus Wulf. in Jacr. coll. 2. r. 14. fig. 2, ynoad viva exconplaria Auctoris. Etiam e Finmarkis habco. Hercyn. - Goti.)

6./. T. sulphurea, crustacea verrucosa sul. phurea; scutellis velatis convexis nigris. Pl. lich. t. 20. fig. 2. 
Iu moutosis rupestribus, saxis. (Crusta oc. currit etiam sordide vel e ferrngineo lutescens.)

65. v. flavo - virescens, crustacea Havovirescens; scutellis fusco - anrantiis flavo. marginatis. Pl. lich. t.20. fig. 1. (Patel. laria (avo-virescens.)

In xupibus, (Carinth. Wulf. Lich. Ravo - vi. rescens; Jacq. coll. 2. 1. 14. Jig. 5. a.)

*66. v. salicina, leproso-crustacea fave. scens; scutellis aurantiis margine pallidio. iibus.

In cortice Salicum. (Lich. salicinus Schrad, spicil. 32.)

*67. v. vitellina, leproso-crnstacea vitellina; scutellis concolorilus. Pl. lich. t. 26. fig.2. (Patcllaria vitellina.)

Ad ligua sicca, asscres. (Ad rupes scutellis acrustaceis sparsis miuntis: V. auerella. Crusta vitellina, scutcllis lividis sordidis V. arcuata 12l. lich. t. 1\%. Jig. 2. Cnllsta parasitica? - ni. gra, leproso-graunloso scutellis sparsis minutis vitellinis V. byssina. Enum. bich. 1.4. Jig. 7 . Lich. byssinas,) 
193 CTIPTOCAMIA SCUTELTATA

* f3. v. cicrira, leprosa pulverulenta ciuina; scucllis amrantiis numutis favo.marginatis.

In sixis, mpibus. (In ligno: ecrotellis fulvis crusta lcprosa citrina P. candelari similis, V. fulve. Cansta pulverulenta llava; scutcllis mi. nutis sparsis lividis V. Rava. In cortice. Cru. sta farinosa tenuisima aurca, flavisgima, citri. na ligna, scpimcula saxa operiens, in qua nun. quam hucusqu e scutelias observare potui, mi. croscopio : 11 becta differre a prioribus ct alio generi subjicienda videtur. H. 1. Lepra cande. laris EFrh. crypt. exs.)

69. v. cruenta, crustacea tartarea rimo. so - verrucosa liavescens; scutellis plano. conrexis marginatis cruentis. $P l$. lich. t.27. fig. 1.

In mollosis rupestribus (iJercyn. - crusta occurrit fusco - Ravescens, albida. H. 1. Lich. ventosus 3jiclis. fosrt. sicc. brit. 5. 23.)

$70 . v$. Haematomma, leproso-crustacea al. bida; scucllis diffurmilus cum crusta eleratis sanguineis. $P l$. lich. t. 40. flg. 1.

In rupibus, saxis montosis. (H. 1. Jich. coc. cineus Ilichs. Tort. sicc. brit. 5. 24.) 
* V. porpliyria, leproso - crustacea albida: scutellis marginatis saturate sanguineis. $P l$. lich. $t .51$.

In rupibus, saxis. (H. 1. Lich. porph)rius Pers. in an. bot. 11. 17. A priori rix nisi marginis regularitate diversa.)

* v. fronlosa, crustacea farinosa expansa viridi . llavescens; scutellis coccineis margine crusta concolore farimoso cinctis. $p l$. lich. t. 49. fig. 1.

In saxis. (Occurrit Crusta cinerea albida dj. lute flavescens. Scusellae margine farinoso cin. ctae. H. I. Lich. coccinens, saxatilis late effu. sus, crnsta farinosa Icprosa llava; scutellis im. mersis diffurmilus coccineis Ters. in an. bor. 11. 17. Absque scutellis vid. 11. 1. Lepra sulphu. rea Ehrh. cryth. exs. 208.)

* 71. v. geographica, crustacea rimosa tessellata Havescens; scntellis depressis nigris contignis. Enum. Lich. t. 5. fgg, 1. (Lich. geographicus.) Pl. tich. t.54. fig.2.

In montosis rupestribus, saxis. (Occurrit Areolis variae magnitudinis, aliis minutisinis vix nudo oculo distinguendis, aliis majoribus osel. 
ocellatis; dispersis aliis minutis : V. atro-2.irests, crusta sparsa favescente margine scutellisque sigris. $\Gamma l$. lich. 1.17. Jig.4. - Quoad frucus couformationem accedit ad Opergraphas, thecis vero stupac filamentosae aduatis, scutellis cru. stae adnatis ad Licherles.) 


\section{N D E X.}

\section{A.}

Acrostichum longif. 6 marantae

seprentrionales 2 Afzelial cirrita crisp.a

herinmalla p:ssilia

Andieata Andree petroplila

Anthocerns

laevis

32

5.4

35

ib.

80

ib.

ib.

94 ib.

Asplenium punctatus ib. 12

Adiantum lanc. ib. nigrum ib. alternifolium 15 Ceterach

ib. germanicum ib. Rutumuraria 12 Scolopendrium 11 septentrionale 18 Trichomanes 15 viricle Astmoos

\section{(1)} (1) 
Bryum brovicaule 36 caespititiun 50 canescens 41 cupillaceam 42 cirratum controversum $5 / 4$ convolutum 43 cribrosmm 51 crispatunn 52 cuneifolium 45 curvirostrum52 cuspidatum 52 delicatulum 5 . Dickroni 32 Didymodon is elongatum 41 elongatum 48 extinctor. 26

$$
\text { n. 1.2. sup. }
$$
fallax 4 if fasciculare 42 fasciculatum 28 fastigiatum 40 flagellare 57 flexwosum 38 fragile ib. glaucum ib. heteromallumib. heterostichum hypuoides 42 juniperifol, 40
Bryum lanceolat. 31 lanuginosum 12 laterule $\quad 5 / 4$ lizulatum ib. lonivicaule 36 longifolium 39 lutcolum 5.4 microcarpum 42 microcarpum ib mucronulat. 44 murale 4is nervosum 44 nigratum 53 nigritum ib. nutans orale pallidun palndosum 34́ parvulum ib. pellucionm 35 pendulum 50 polycarpum 37 polyphyll. ib. poiniforme 54 pulvinatum 31 punctatum 52 purpureum 56 pusillum 55 pyriforme 27 recurvatunz $3 \mathbf{x}$ recurvum 5 4 Bryum 

$\begin{array}{cc}\text { Bryum rigidulum } & 40 \\ \text { rigidum } & 46 \\ \text { rivulare } & 37\end{array}$

ros+ixin

rubellun

rugosum

rurale

scoparium

seriatilu

setaceum

selicelim

simplex

spuriun!

starkianum 52

stellatum $\quad 45$

strictum $\quad 36$

strumiferum it).

subulatum 46

sortuosum il).

trichodes 47

triquetrum 48

truncatulum 27

undulatum 40

unguiculat. 45

ventricosum 49

virens

virens

viridulums

riridulum ib.

Wei]sia 33
Buxbaumia

21

aphylla ib. foliosa ib.

Buxbaunie ib.

C.

Catharinea

hercynica ib.

Cladonia

114

attenuata 125

Jiuncialis 116

botrytes $\quad 128$

ceranoides 126

coccinea 123

cornuta 119

cornucop. 128

coronata $\quad 125$

cristata 124

deformis 120

denticollis 125

digitata 224

dilatata $126^{\circ}$

elongata 119

exilis 121

extensa $\quad 225$

Filula 127

fimbriata 122

foliacea $\mathbf{2 3}$

furcata, 15

furcato.sub. ib.

o Clado. 
Cladonia furcell. 118 furcell.rad.120 fusca 127 gracilis

hybrila

119 incana ib. irregularis 125 macilenta $\mathbf{1 2 6}$ marginalis 123 molariform.117 Papillaria ib. parasilica 127 phyllophora 123 polycerliala 126 polyceras 120 prolifera 122 pyxillata $12 x$ racenosa 114 radiata 120 rangiferina 114 rangiformis ib. recura 115 simplex 121 spinescens 115 spinosa ib. squamosa 125 subulata 118 subuliformis ib. sylratica 114 taurica 118 tubieformis 122
Cladonia tubercul.122 turgida 124 uncialis 117 uncinara 116 ventricosa 126 verticillata 122

Collema auriculat. 98 argillosum 102 Botrytis 103 by fsinum 105 ciliatum 104 conglomer. 102 corniculat. 105 crenulatum 101 crispatum 100 crispum 101 cristatum ib. fasciculare 102 finbriatum 104 glaucescens 100 Graniforme 106 granulosum 99 Taciniatum 100 lobatum 105 marginale 101 nigrum $\quad 103$ oliscurum 101 paunosunb 105 polycarpon 102 prasinun 96 pulvinatum 104 Colle. 
IṬDEX.

Cullema subtile 105 tenuissimún 10 á tomentosum 99 $V$ espertilio 98

Coralloides acul. 142 fragile, 131 globiferum $\mathrm{ib}$. pascliale. ib.

Connicularia trist. 142 Cyathea

10

\section{D.:}

Dicranum acićnl. 37 aquaticum 35 nagellarc 37 heteromall. 58 longifolium 59 ovale 35 polycarpum 37 p!sillum 5 ; scinroides 67 sp:rium 39 strumifexum 37 undulatum 39 varium virens

Didymodon capill.42 rigidulum 40 Ditrichum pusill. 43

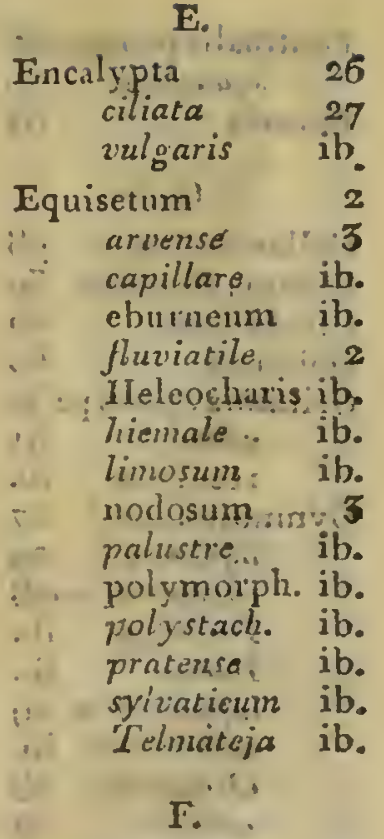

Fifsidens adiant. 56 bryoides? 55 $\begin{array}{cc}\quad \text { polycarpens } & 37 \\ \text { strumifer } & 36 \\ \text { Fontinalis } & 79\end{array}$ $\begin{array}{cc}\text { polycarpens } & 37 \\ \text { strumifer } & 36 \\ \text { Fontinalis } & 79\end{array}$ $\begin{array}{ccc}39 & \text { polycarpers } & 37 \\ 35 & \text { strumifer } & 36 \\ \text { ih. } & \text { Fontinalis } & 79\end{array}$

antipyretica ib. minor. $\mathrm{ib}$. minor .. 80

$\mathrm{O}_{2}$ Fonti- 
Fontinalis pennata 50 Funaria

79 51

\section{G.}

Gallertfechte Georgia Mnemos. 30 Glockenluth

Golt!har

98
30
26

Grinimia apocarpa 50 cribrosa $3 x$ recurvata ib.

Gymmostoinum 27 - aquaticum 29 curvirostrum28 Hedrvigia ib. Heimii ib. làpponicnm 29 nicrostom. ib. Osmundac. 28 oratum 27 pennatum 28 pyriforme 27 stuncatulum ib.

\section{H.}

\section{Iraatfeclite}

152

Hedwigia anodon 29 aquatica ciliata
HYýpnum $\begin{array}{ll}\text { abietinum } & 5 y \\ \text { adiantoides } & 55\end{array}$ adluncum a) fine albicans alopecurum 70 aquaticum 29 attenuatum 67 brevirostrum 76 bryoides 55 conmulatum 59 complanat. 57 compressum 58 compress. ib. crispum il). crista castr. 62 crista castr. ib. cupressif. 63 curtipendul. 66 crsspidatrm 58 cusp.inerm. ib. decipiens $7 \tilde{J}$ delicatum 50 dendroides 69 denticulatum 56 dubium $7 x$ elegans $\quad 58$ extenuatum 63 extricatum 62 filicinum 61 Hypnum 
Hypnum filicinumbis filiforme, $7 x$ Aluitans 78 fontinaloides 79 gracile 68 Halleri , 8 Halleri ib. hetcrophyll. 66 illecebr. 58,69 intricatum 72 intricatum 76 lacunosim 63 longirostr. $\quad 76$ loreum $\quad 66$ lucents $\quad 56$ lutescens $\quad 75$ medium $\quad 67$ molluscum 62 murale $\quad 76$ muticum 58 myosurvides 69 nirosurum ib. nitens palatinum palustre parietinum $5 ?$ pennatum 57 piliferum 75 plumosum 73 plumosum 74 polyanthes, ib.
IIypnum polycarp.70: praclongum 72 prolifernin 59. ponliferum ib. prolixum. 78 purum . 58 putum ib. ramosinn 75 re/uscens, 59 riparioides 78 riparium ... 77 i rivulare … 78 rugostum 63 ruscifolinm, 77. rusciforme: 78 rutabulum 77 salebrosum $74^{\prime}$ Schreberi, 58 sciuroides: 66 scórpioidas, 63 sericeum ㄱ 74 serpens in 72 spleudens: 59 squarrosim 65 squarrosum 64 stellatum 65 striatum , 75 . strigosum 76 subtile 70 sylvaticum. 56 taxifolium: 55 O 3 Hy- 
Hȳpnum trichodes 59 trichomanoid. 56 triquetrum 59 umbratum ib. uncinatum 62 undulatum 57 undulatum ib. velutinum 73 viticulosum 67 viviparum 69 Hypoxylon locul. 137

\section{I.}

Isidium Westr. 129 frigidum 169 Isoëtćs lacustris 2 Jüngermannid 81 albicans $\quad 8 / t$ alpina 80 angulosa 90 asplenioides 37

$\because$ barbata bivornis

bicuspidata bidentata complanata concinnata ciliaris ciliaris" dilatata otrarginata 81
Jungerm. tpiph. $9^{2}$ excisa ỡ furcata 91 julacea 82 lanceolata 88 mullifula $9^{1}$ multifida 90 nemorosa 85 pallescons 87 pinguis 91 platyphyllía 87 polyanthos 88 puloscens 91 pulcherrina 85 pusilla $9^{\circ}$ pusilla ib. quadrident. 8 quinquedent. ib) radicans 87 reprans 8 in resupinata 8 scaluris $\quad 89$ setiformis 8? spliagni 83 sizina 86 tamariscifol. ib. Tomentella 85 tomentosa $\mathrm{Q} 1$ Trichomanis $n$ trichophylla 8: trilobata 87

Junger- 
INDEX.

Jungerm. undul. 85 Lichen aculeaius 142 viticulosa 88 allustus $1 / 45$ agigregatus 331

\section{K.}

Kahlmund

Kandelivisch

Koelreutera

Konallenleclite $\quad 328$

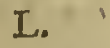

Leersia ciliata

lanccolata 31

vulgaris

Leskia acienuata 67 longifolia 75 marclica 54 paludisa 64 polyantia 74 polycarpa 70 sericea 75 subtilis 70 ıichioman. 57 vivipara 69

Lepra farinosa 171 lutescens 195 candelaris 198 sulphurea 199

Lichen abietinus ig 3 Acetabulumi47 alabsastrin. 168 albellus 172 albescens $\quad 165$ albido-caes. 172 albo-ater 193 albo-cuerul.189 allo-flavesc. 170 all,o incarn. 176 aipolius $\quad 152$ allochrous 155 ambiguus $\quad 355$ ambignus 152 amplissimus 106 anylaceus 1.94 androgynus 73 angulosus 171 angustatus 153 ammulatus 189 anthracinus 110 antiquitatis 187 aphthosus 107 aquaticus $\mathbf{3} 48$ arcuatus 107 arenarius $13 \%$ arenarius 178 $\arg \cdot$ : fuscus 181 - articulatus 1.55 atro allus 132

04 Lichen 
Lichen atrenuat. 125 ater 183 atratus $\quad 183$ atro-cinereus ib. atro-sang. $1 g^{2}$ atro-zirens 200 atro - vilens 196 argillosus 102 aurantiacus 278 aurcllus $\quad 197$ aureo-cerin. 179 nuriculatus 98 badius $\quad 182$ Bacomyces 176 barluatus $\quad 152$ bicolor $\quad 135$ biuncialis 110 botrytes 128 Botrytis 105 bracteatus 169 brunneus 167 bryophilus 164 byssinus $\quad 305$ byfsinus 197 caelatus 166 caesio. ru fus 178 caesius $\quad 156$ caerulescens 183 caerulescens ib. valearius . 185
Lichen calcivorus 387 calycaris $\quad 359$ cancriformis $17 \mathrm{I}$ candelaris 159 candicaus 164 candidus ib. candidus 165 canescens 165 caninus $\quad 106$ caperatus 1488 capitellatus 3044 carnosus 161 centrifugus 154 ceranoides 116 cerinus 179 chalybif. 135 cicatricosus 13.1 ciliaris 194 ciliatus 104 cilintus $\quad 155$ cinereo-ater 132. cinereus 185 cinerens 194 circinatus 157 cirrosis $\quad 115$ citrinus $\quad 158$ citrintes $\quad 108$ citrinas ifis citricolorus 153 elausus 
Lichen coccineus 123 coccineus 195 cochleatus .99 concolor $\quad 159$ condensarus 150 confinis -ib. confluens 18 ' contiomer: 122 conglomer. 174 contiguus 184 contortus 186 coracinus 385 corallinoid. 129 corallinus ib. corniculatus i 41 corniculatus 105 cornucop. 123 cornutus 139 coronirus 125 coronatus 175 corrosus 111 corrigatus 1-17 corrugatus 132 crassiformis 1 fo cressuss ib. crenulatus 102 cretacens 185 cribellum 211 crinitus $\quad 112$ crispatus 100
Lichen crispus 101 cristatus 124 cristatus 101 cristatus 100 cruentus $\quad 198$ cucullatus 143 cupularis 176 cyanescens 189 decipiens 162 deauratus 166 decolorans 177 deformis 120 delicatus 127 dendriticus 168 denticollis 125 detritus $\quad 172$ derisurs 110 dichoromus 134 denudatus 141 diffusus $\quad 156$ diffusus $\quad 155$ diffusus $\quad 156$ digitatus 124 dilaceratus 140 dilatatus 126 discoidens 170 dispersus 180 divaricatus 154 dubius $\quad \mathbf{1 5 6}$ effusus $\quad 274$

\section{5 \\ Lichen}


Lichen elongatus 119 elveluides 162 encaustus 154 endivifol. 128 epigeius 164 erosus

111

escharoides 191

exanticn1. 177 exilis

121

exbinsils 125

fagincus 170 fahlunensis 154 fallax $\quad 1.19$ Jarinaceus $13 y$ farinosus 171 fascicularis 102 fascicularis ib. fasciculatus 190 fastigiatus i yo ferrugineus 177 Filuela 127 funbriatus 10.4 fimbriatus 122 Aaccidus 153 flavescens 159 flavidus 157 favo-rub. 278 navo.vir. 179 favo-vir. 197 Bavo-fusc: 130
Lichen flavus 198

flocculosus 110 floridus 135 furviatilis 148 foliaceus 123 fragilis 132 frandulent. 149 Jraxineus 158 frigidus 1.8 frondosus 1.99 fucinus $\quad x+2$ fucoides ib. fulgens $\quad 165$ Suliginosus 109 fulvias $\quad 2$ go fumosus 190 furcroto-suli115 furiatus 1 i. furc.-rad. 120 furcellatus 118 $\int u r f u r a c c u s ~ 144$ fusco-ater 181 fusco $\cdot r u b \cdot 175$ fusco - xub. 176 fuscus $\quad 127$ fuscus 181 fuscus 154 geograplic. 199 gelidiform. 362 gilvus 
Lichen glaber 109 glauccscens 100 Glaucomr 172 glaucus globiferus 51 glomulifer 148 gracilis grandis graniformis 106 gianilosus 9.9 grauzisosus i75 griseo-ater 182 griseus $\quad 167$ grisens , 111 griunosus $\mathbf{1 8 8}$ guttatus $19^{2}$ gypsophilus 162 Hatmatomilz. 198 herbaceus 147 hippotrichod. 137 hispilus 152 hiirsutus $\quad 112$ hirsutus $\quad \mathrm{I} 3$ hirtus $\quad 133$ holocarpus $\quad 179$ loorizontalis 107 Tumosinalis $19^{\circ}$ humosus $19 \mathrm{~g}$ lyperboreus yo hyzridus $\quad$ II9
Lichen hypnor. $x 66$ lypnorum ib. immersus $\quad 187$ implexus $\quad$ 13/4 impolitus $\quad 172$ impressus $186^{\circ}$ iincenus 121 incoloratus 177 incurvus $\quad 157$ intricatus $\quad \mathbf{5 6}$ irregularis 125 islandieus $\quad 143$ jubatus $\quad 134$ Jungermann. 179 juniperinus 145 Koenigii $\quad$ I88 lacteus $\quad 169$ laciniatus 100 laciniosus 147 lanatus $\quad \mathbf{3 6}$ laneus $\quad 135$ laqucatus 160 lentigerus $\quad$ I6/ leucophlaeus 173 limitatus 192 lobatus $\quad 105$ - lobatus $\quad 150$ luteolus $\quad 175$ lutesccris $\quad 19^{5}$ masilentus 126 
Lichen maculif. I $9^{5}$ madrueporif.i2g madratporif.117 marginalis 123 marginalis roI mnimoreus $1^{-6}$ marmorens 187 melanocarp. I $5 \mathbf{t}$ melauocarp. I 64 mesenterif. II 2 microphy-llus 567 miniatus $\quad \mathbf{5} 88$ molariformis 117 multifidus $\quad 157$ multillorus 167 mulipunct. 154 multimnactus 185 muralis $\quad \mathbf{5} 7$ murorum 158 muscorum IOI muscorum 16 年 mroclurous 99 nebulosis $\quad \mathrm{6} 60$ niger 12 ? niger 103

nigricans 143 nivalis ib. nodulosus $\quad \mathbf{1 7 0}$ obliteratus 178 ocellatus 185
Lichen ocellatus 186 ocellulatus 183 ochroleucus 158 obscurus 155 obscurzis IOI oclirolcucus 137 nculatus $\quad x^{29}$ Oecleri 1083 olinacesss $1 ?^{2}$ olivaceus 150 omphalodes 145 ompladodes 153 orbicularis $\mathbf{1 5 5}$ orbiculatus 170 ostreatus $\mathbf{1 6 3}$ pallescens 49 pallesc. $17 \mathrm{r}$ 196 pallescens $\mathbf{1 5 6}$ pallide fusc. $18 \mathrm{r}$ pallidus $17 \mathrm{r}$ pallidus 175 promosus 103 pantherinus 184 Papillaria 117 papyraceus $\mathrm{I} \cap 8$ paralloxus if 63 parasiticus $\quad \mathbf{1 2 7}$ parictinus 150 paschalis 130 patellatus 147 
Lichen pellitus Ir 3 Lichen Psora $\quad 56$ Perellus $\quad \mathrm{x} 9$ perlatus $\quad \mathrm{I} 48$ pertisus is r pertusus $\quad 186$ petraeus 18: pezizoides 166 phyllophorusr 23 physodes $\quad 150$ pilularis 193 pilularis ib. pinastri $\quad \mathrm{i} 6$ plicatus $\quad 132$ plumbeus 146 polyc. $159-160$ polycarpus 102 polyccpl:alus 126 polyceras 120 polydactylosio6 polyphyllus ro9 polyrhizos 113 polytropus 196 populinus $\mathbf{1 4 0}$ porphyrius 199 prasinus 98 proboscideusir 2 prolifer 122 pruiuatus 172 pruinosus $\quad 176$ prunastri 190 pubescens 136 puichellus $1 \leqslant 6$ pulcher $\quad 8$ pullus $\quad 150$ pullus $\quad 5.4$ pullus Ix pulinonarius 46 pulceraceus 153 pulveralentusi 52 pulverulent. 153 pulviuatus 10 f punciatis 185 puretatus a rig punctiformis ig 3 purpurascens 187 pusculaus rII jyxidatus I2I quercifolins 149 quercinus it. querneus ins quisquiliaris iso racemosus 11.4 radiatus 120 radiosus $\quad 157$ ramulosus $15 \mathbf{1}$ ramulosus $16 \mathrm{r}$ ramulosus $\quad 135$ rangiferinus 314 rangiformis $\mathrm{ib}$. 
Lichen recurvus 115 ravidus 180 resupinatus 108 rimosus 185 rigidus $\quad 112$ rigidus 154 rigidus rosellus rubcllus ruber

rufescens rilfescens

saccatus salicinus

salignus sanguinarius $19^{2}$ sanguinarius 194 saturuinus 99 saturninus saxatilis saxicola scaber 99 145 scruposus semipinnatus $15 \mathbf{r}$ semi pimnatusib. sepincola

145

$$
\text { serratus }
$$$$
147
$$$$
\text { setosus }
$$$$
137
$$

silacerss

187 similaris
Lichen simplex sordidus 121 spadicens 172 spadochrous in 3 speciosus 153 spladeroides 174 spinescens 115 spinosus squamatus il). squamosus $\quad 125$ squarrosus 150 stellariform. 15: stellaris ib. stillicidior. 180 stygius $\quad 154$ subjuscus 180 submbricat. 157 subtilis 105 subvirescen's 37 subulatus ir subuliformis ib. sulplutureus $19^{6}$ sylvaticus 109 sylvaticus 109 symplyycarp. 27 tartcreus $\quad 173$ rauricus 118 tegularis $\quad 158$ telluissimus 142 tenuissimus 103 
Lichen tephrom. 183 terebratus $\quad 15 \mathrm{I}$ tessellatus 185 testacens $\quad 162$ tigriums $\mathbf{1 8 5}$ tilinceus $1 / 49$ tinctorius 14 tomentosus 108 tourentosus 99 tremelloides 104 tristis tubaeformis 122 tuberculosus ib. tuberculosus 129 inberculosus 170 tumidulus ib. turbiuatus $16 \mathbf{1}$ turgidus aliginosws ulmi umbilicatus $17 \mathrm{x}$ unbrino o uss. $18 \mathrm{r}$ zmbrinus ib. uncialis $\quad 117$ uncinatus 116 upsalieusis 168 varians

172 variabilis167-168 varius vellews
196 II 3
Lichen vellens 114 venos:s - Iก7 ventosus Igó ventricosus 126 vermicularis 118 vernalis .175 verrucarius 146 verrucosis ib. versicolor $\quad 157$ verticillatus 122 vesicularis . 165 vespertilio 98 viridis . $19 \mathbf{I}$ viridi-flavesc. 174 viridi - rufus 179 vitellinus istivinus

197 Lichenoid.ciliare I4/4 corn. bronch.14 furfuraceum 144 lispidum $15 \mathrm{r}$ islandicum! 145 Lobaria 138

Acetabulum 147 aculeata $\quad 142$ adusta $\quad 145$ aipolia $\quad \mathbf{1 5 2}$ aubiguta $\quad \mathbf{1 5 5}$ angisstata 153 aquatica 148

Lobaria 
Lobaria caesia 156 Lobaria muralis 157 calycaris $\quad 130$ candelaris $\quad 359$ caluerata centrifuga ciliaris

nizalis

1. $j^{5}$

olinacea $351)$ omphalodes 1.45 orbivularis $\quad 155$ pallescens ri, 6 parierina is perlata physorles I 43 pimastri 350 polycarpa I59 populina - so pramastri ib. pulla $\quad$ ifí pulmonaria sa 6 pulveracea 353 pulverulenta 352 radiosa $\quad 157$ rigida $\quad 1.42$ saccata $\quad 147$ saxatilis $\quad 1 / 45$ saxicola $\quad 158$ semipinnata $15 \mathrm{r}$ scpincola $\quad 345$ speciosa $\quad \mathbf{5 3}$ squarrosa $\quad$ i5g stellariformisi 52 stellaris ib. stygia $\quad 354$ tegularis $\quad \mathbf{5 8}$ tenuissima $\mathbf{1 4 2}$ Lobaria 
Lobaria terebrata 151 tiliacea

tillctoria.

149

verri:cosa $\mathbf{1 4 6}$

versicolor, 157

Lycopodium

alpinum

annotinum ib.

clatatum ib:

complanat. ib.

denticulat. 17

helveticum ib.

inundatum $\mathbf{1 6}$

radicans ib.

selaginoidles ib.

selago ib.

M.

Marchantia

$9^{2}$

hemisphaeric. ib.

polymorpha it.

conica ib.

Marsilea

matans

1

ib.

Marsilie

Meesia longiseta

Mrium
Muium androgyn. 46

annotinum 49 argenteum $5 \mathrm{z}$ bimum : 48 caespititilim 50 capillare ib. carneums $5 \mathrm{~s}$ cruduin - 48 cuspidatum 52 ellipticum ib. fissum 39 fontanum 54 holosericeun $5_{1}$ horruin $\quad 48$ hygrometric. 50 luterale 54 marclicum 55 mutrans 49 osmundac. 28 palustre 47 pellucidum 30 P'ohlia 48 pomi, orme 54 prolixum 49 peudotrig. ib: purpureum 36 pyriforme 50 ramosum 49 roseum 53 rostratum 62 
Muium erpillif. 52 setacelim squarrosum 47 stellars 51 Timmia 53 triclodes

Triclıoman. 90 triquetrum 47 turbinatum 49 undulatum 55

\section{N.}

Nabelneclite

Nidelschorf

Natterzunce

Necliera curtipend. 66

penuata

pumila

viticalosa

\section{o.}

Ohnmund

Orroclea

cricpra

sencibilis

spicant

serutliopterisil.

Ophioglossum

$$
\text { v'ulyatum }
$$

il.

67
Orthotriclum

25

affine anomalum 25 crispitm ib. cupulatum 26 diaphamen ib: liercynicun 25 striatum ib.

Osmunda $3 / 1$ crispa 11 Lunaria 3/4 ramisa ib. regalis ib. spicant 11 struthiopt. ib.

\section{P.}

Paludella

Patellaria candida 164 Perella 1 fig rubia 175 decolorans 177 ferruginen ib. cincreo-fusc.ib. ceriua sciuposa 186 silacen 137 navo-vir. 197 allio caermt:iog "psalicusis 168 Patel. 
Patellaria raria 196 Pilularia vitellina 197 globulifera muscorum 164 Placodinm 155 zzebulosa vesicularis 163 Peltigera ampliss. 106 aphthosa 107 canina 106 horizontalis 107 papyracea 108 poly.lactyl. 106 rufescéns 107 sylvatica 109 tomentosa 108

Phascum venosa 107 axillare $\quad 20$ crispum, ib. curvicollum il). cuspiclatun 19 muticum 021 nitidum 20 patens ib. piliferum 19 serratum 20 serratum ib. stoloniferumib. subulatum 19 velutinum 20 Pillenkraut 1 Platisma aquatic. 148 caperatum ib. denudatum 141 fallax fraxi $\begin{array}{rr}\text { sepincola } & 1 / 5 \\ \text { Pohlia elongata } & 48\end{array}$ Poly podium aculeatum \& alpinum $\quad 10$ angustatum 9 anchriscifol.ib. appendiculat. 8 aristatum ib. Callipteris 6 $\begin{array}{ll}\text { canibricum } & 4 \\ \text { cristatum } & 8\end{array}$ cynapifolium 9 dentatum 7 denticulatum 9 dilatatume 7 Dryopteris 10 Filix femina 6 Filix mas ib. fontalum 10 fragile 9 ? P 2 Poly: 
Polypodium incis. 7

Ionchitis 4 molle 7

montanus 10

mout.111121 ย́

Oreopleris ib. pedicularif. so

Phegopteris 5

regiums

1)

sliaticum ib.

rigigielum

ranacelifolinm8

tenue

Thelypteris

9

1 trifidus

vulgare

Polytichum

alvides

communne

rricoides

bercinicum

juniperifol. 24

juniperifol. qu

joniperimum $\approx$ f

nomutat

ib.

มลเกาม

piliferuni

subrotund.

modulistum
$2 j$

2.

25

(f)
Folytricl. urnig. 24 yllcraefol. 23 Potua cavifolia 27 curvirostra 28 cust. major 27 - minor its.

Psora pyriformis 28 161 aknhastrina 368 alli:scens. 165 mimbigna 155 bracieata 1 Gig lonumea 367 cuelata $\quad 166$ caesia $\quad \mathbf{1 5 6}$ candida 164 canescens 165 carnosa $\quad 161$ citrina $\quad 365$ deaurata 166 - decipiens 162 dendritica 368 epigeia 164 frigida 168 selisliformis 162 grisea 167 gypsophila 162 hypnorum 166 lactea ientigera
169 164 
Psora micropliylla 167 murilis 158 mis.scorum 164 nelvulosa 166 ostrentu $\quad \mathbf{1 6 3}$ paraloxa ib. pezizoilles 166 saxicola $\mathbf{1 5 8}$ similaris 163 squamata $16 \mathbf{z}$ cestacer $\quad 162$ turlinata ${ }_{16} 6 \mathbf{x}$ upsuliensis 168 variabilis 167 vesicularis afio

\section{Pteris}

aquilina 12 Pterigynand. filif. 71 grảcile

Pulmonaria lobat. 47 reticulata 346 veruucosa ib.

\section{R.}

\section{Riccia}

minima

09

ciliata $9^{5}$ caternosa ib.
Riccia bifurea $9^{5}$ slanca ib. ryramilata of jluitaris it). canaliculata ib. naicans ib. Pollfarm $\quad 12$

S.

Salvinia

natans

1

Salvinie it.

Sannifirm 11

Schildflechte $\quad 106$

Sclenriflechte, 1 fie

Scolopendrium 1/f Sphagnแm - 21 acnifolinm 22 cuspiclatum il\%. intermediutnib. ohtrisifotium 21 tencllim 22 Sphacriabysancca 104 Splaclumm ib. amprullaceum 2.3 froclichinn, ih. ligulatum il.. sphaericum

$$
\text { P } 3 \text { splach. }
$$


Splachumm tenue 23 vasculosum 28 Squamaria centr: 154 fahimuensis ib. juniperina 145 pinastri

pulla pulverul.

3.46 stygia

Stantmions

15 ' 132

154

Stereocaulnn 30

comlensat. 150 confine ib. Corallina 129 corallinoialesils. fragile $35 \mathrm{x}$ slobifermu ib. madrepror. $1=9$ praschate 130 quisquiliare ib.

Stemmoos 4 f

Strichfarru

Strunklleclite

12 Swartzia capillac. 42

\section{T.}

Targionia

liypophylla ib. Tetraph is

5o pellucilla ib.
Timmia megapol. 53 Torlmoos $\approx 1$ Tortula tortuosa 46 Tranbenfarm 14 Trentepolilia $\quad 17$ erecta il. Tremepolilie ib. 'Trichost. angustat. 40 cunescens $4 \mathrm{t}$ clongratum ih. ericoides ib. fasciculare 42 fontiml. 79,30 fietcrostich. 40 lanuginos: 41 pallidum 45 pusillum ib. ramicerum 42 serratum ib.

Tristichis Tubercular.fusca 127 Tü parasitica ib. Tüpfolfarin

\section{U.}

Unbilicaria 109 anthracina 210 cirrosa 115 corrugata 112 crinita ib. 
Umbilicaxia erosa 111 flocculosa 110 orrisea hirsuta 111 hyperborea 110 polyoligila 109 puitulata $11 \mathbf{1}$ rigiila

112 spadoclirca 115 vellea

Usnea

articulata 133

barbata $\quad 132$

bicolor $\quad \mathbf{3 5}$

chalybeif. ib.

dicisoloma 134

flaccicla 133

florilla ib.

hippotrich. 137

hirta

iurplesca

intricata

juliata

lanata

lanéu

ochroleuca

plicata

pubescens

ramulosa

vulpina
153

154

156

134

136

155

157

152

136

135

138

\section{V:}

Verrucaria

alictina $19^{3}$

albilla 171

albo-atra ib.

albo-caerul. 189

amylacea 194

angulosa ii). aubulata 189 arcuatu $\quad 197$ arenaria ib.

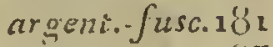
atia

atrata

105 airu.alla 1 \&2 atr. sangriin.1?2 atro-viruss 199 aurantiaca 187 aurella : 297 aureo-cerina 179 badia $\quad 183$ byssina $17^{7}$ caerulescriss 188 caesio-rufa 178 calcuria $\quad 185$ calcizora 187 cancriformis 171 capitcllata 394 cerina

279

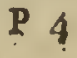

Verru- 
Ferruc. cicatricos.134 ciner. atra 182 citrina $\quad 398$ clansa $\quad 177$ confluens 384 conglomer. 174 contigua ib. coniorta 186 coracina 183 coronata 175 cruenta 198 cupularis $\quad 176$ cyanescens 139 decolorins 177 dendritica 168 detrita 172 discoiclea $17 a$ dispersa 180 , effusa 174 escharoides 194 faginea 370 farinosa 172 frisciculata 190 ferruginea 177 Sal'a $\quad 108$ Havo- fusca 180 llavo.viresc. 107 $\begin{array}{ll}\text { fulva } & 198 \\ \text { fumosa } & 19 a \\ \text { fusca } & 18 !\end{array}$
Verruc. fusco atraisi fusco rubel.175 frondosa 299 geographicalg9 gilva 179 glaucoina 172 gramulosia 175 griseo-atra 182 grumosa $\mathbf{1 3 8}$ gutlata $22^{2}$ Hizenatom. 3 cys holocarpa 179 humosa 291 humosimilis 1 no immersa 187 impolita 172 incolorat.x 177 Jungerman. 179 limitata $\quad 192$ lutescens ing maculiform.ing mult ipuncta 185 muscorum $29^{2}$ nodulosa 270 oblitterata 173 ocelluta 183 ocellulata ib. Oedleri $\quad 188$ olivacea 192 orbiculata 370 
Verrucạria pallid.171 pallide fusc.18 $8 \mathbf{r}$ pantherina 384 Porella petraes 169 pilnlaris 193 polytropa 196 porphyria 199 punotita 192 punctiform. 193 purputirasc. I8̂7 quernea. ravirla rosclla rubra rubella. rufescens rugulosa rupestris salicina 195 180 176 175 17.4 173 170 137. sanguinaria in scruposa 285 silacea 187 stillicition. 180 sulfusca ib. sulphurea $19^{6}$ tartarea 173 tessellata 185
Verrucar. tigrina 185 tuberculosa 170 . tumidala ib. uliginosa Iça unbilicata 171 zmmbr. fusca 18 i varia . ang vernalis 175 viriclis 191 viricli-rufa i 179 . vitellina 197 Vieltheil.Fleclute 338 Vicrzalin 30 W.

WaxzenRcchie - 169 Vebera nutans 49 pyriformis 50 Weissia controv. 54 crispa ib. Dicksoni 32 hetcromalla 35 nigrita ib. prisilla ib: recurvirostr.32 starkcana ib. Viderthon 23 


\section{ADDENDA ET EMENDANDA.}

AVCTONES BOXANICr.

Acharins, Ex. Lichene: - in Kon. Vet. Acad. ' Jya llandl. 15. 1794. :6. 1795.

Andrew's Engraviugs of Icdths - Lond. Fol. 1795 . Bartalini, B. Catal. delie piante - 8. Siena, 1776 . Baner, Fr. Uelineat. of cxotick plants - Iol. Lond. 1796.

Bolten, I. Fitic brit. 2. Vol. 4. Tond. 86 a go. Borkhausen 11. B. Finn der ob. Grafsch. Catzenelenbogen, in lihein. Mag. 1 B. 1795.

Coyte's, D. Hortus bot gipjovicensis - Lond. 14. 1795.

Davies, II. Decicript. of four new brit. Liclicn's - in Trans. Jin. 2. 126.

Forsyth, W. A botanical nomenclator -8 . Lond. 179'.

Frülich, 1. A. De gentiana - 8. Err. 1796.

Goodenough, S. Observat. on the brit. spec. of : Carex - in Trans. lin. 2. 126.

Haworth's Oloservations on the Geuns Mescm. - bryanthcuum - 8. Lond. $179 \%$. Hoy, T. and I. Fairbairn, an acc. of tivo new Geu. of Plants - in Trans. liu. 2. 546.

Kur. 
Klingsohr, J. G. W. de Gcoffroca inermi Erl. 178g. Kunze, C. S. I. Deutschl. crypt. Gerwäihe 8. Hamb. 1796 .

Lee's introduction to Botany - E3. 5. 5. Lond. 179 '.

Lindsay's, I. Acc. of the Germin. and liaif. of Ferrins from. the sced - in Trans. Jiti. 2. 93 ; Macrier, I. F. Verzeichn. dèr östr. Báume 8. Wieni $i 796$.

Maga\%. des Planzenreichs - 5. Erl. 1795. Marati - nnova Raccolt. d'epuiscoli scientif. * frlolog. 18. Vest. 2770. T. 20 .

Micheaux -

Miller, I. F. Cimclia physioa - Fol. Tond. 1796. NToser, H. C. Deutschl. üonomische Flora 8. I cipz. $1 ; 96$.

Ocstrcichs Flora - 8. Wien, 179 t.

Pulteney, R. Descript. of a min. epiphyl. Ly. cop. - in Trans. lin. 2. 505.

Relhan -950

Ret\%, A. I. Flor. scand. - Lid. 2. 3. Tips. 1795. Rölıling, I. C. Dent:chl. Flora - 8. Brem. 1796. Römer, I. I. Garten-Eucyklop. 4. Tub. 1795. Rousscan and Mart. Letters on Bol. - Ed. 4. 8. I.ond. 3794 .

Roxburgh, TY. Plants of the Coast of Coromandel. Fol. Lond. 1795.

Bussel, Al. - Ed. 2. 4. Lond. 2794.

Sower. 
Sowerby's coloured Figures of english Fungt - Fol. Lond. 1796.

Sibthorp, 1. Flora oxoniensis. 8. Oxon. 179ł. Stackhonse, I. Nercis uritaunica - Fol. Loud. $179 \%$

Stillingfleet's, MIiscellan. Tracts; ; Id. 4. 8; Lond. 279.4 .

Stüver - Ed. angl. by Trapp. 4. Jond. 17946: Teesdalc's, R. Plant. cuorac. - in Tranp. lin. 2. 103.

Yelley, T. Plant. marit. icon, pict. Fol. Lat thous. 1795 .

White, G. Naturalist's Calendar - 8. Lond. 1795 Willemet, 1). Flora mauritiana iuch. 8. Lips. 1796.

Wyttenbach, I. I. A. v. Haller icones plantar. Helvet. Fiol. Bern. 2795.

\section{IN PBALTAT.}

P. 2. 1. เก. lege denselben

P. 3. - . - - wic in der Ordnumgsfolge -

- - 6. - - rröfrere

- - 16. - - Gattungsnahmen

P. 4. 1. 2. $\operatorname{leg}$ Lich. miniatus Lin. loco prninatus. 2.5.1.14. - - ich sahe mich genöthiget jene - 
P. 2. 1. 23. Equiset, fliv. del. Bolt, fil. 36.

P.3. 1. 27. - - Telmateja, ad. Bolt. fil. 4.56. (Equis. Auviatile) - Equis. cburneum schreb. Hopye Terb. viv. l.2. 96.) In puludosis prope Gott. Dorste I'ers.

P. 6. 1.4. ad. Bolt. fil. C. 43. (Acrost: Thelypt.) - - 21. ad. Mag. d. I'fl. r. i. T. 1, s. (Pol. Oreop(eris.)

P.6. 1. ult. ad. obs. fronde utrinque pyramidata, piutuis inferioribus sensim brevioribus.) P.7. 1.8. ad. obs. A sequenti differt fructificats hand conllnentiluns. Var. fronde angustiore.) - - 19. Post trifidis ins. dente intermedio pro: mincute incurvo.

- - 21. ad. obs. Occurrit fructificat. biseriatio angustis rinoribus; pimnulis sessilibus; from- de variat angustiore.

- - ult. ad. piumulis pediccllatis.

P.8. 1. 8. ad. Var. crispa.)

- 1.29. P. appendiculat. ad. P. Lobatum Ituds. angl.2. 459 .

r. 9. 1. 16. P. anthriscifol. ad. obs. H. vid. 1. F: thaticum Bolt. fil, t. 45 .

P. 12. Asplen. Rut. mur. ad. obs. occurrit varie.

tis frondibus et foliolis multo angustioribus.) P. 10. ad. 2s. Polypodium robertianum, fron. de triaugulari, foliolis tenis bipinnatis : pin. is pinunlisque inferme pinuatifidis. 
In moutosis, rupestribus. 11ur. (Gott. Polypod. Dryopt. simile. Stipes glaucus uno latcre sulcatus. Frous vencra. Vterque mudo oculo 6ubtili tomento, ad lentem brevissimis Glandulis uivitus. Olor debilis Geran. robert. Fructif. minuta.)

P. 14. A. Scolopendr. ad. Eriord. Bermliardi. P. 17. Trenecpolilia ad. Hercyı.

P. 19. Pluasc. cuspid. ad. var. miuor.

P. 20. - - patcus, ad.

- - mitid. ad. obs. A Ph. subulato sta: tura minori cante distingucndum.)

P. 22. Buxb. foliosa, ad. Webera Dipluyscium Ëhrle. Eeicr. 1. 177.

P. 22. Splachn. sphaericum, ad. obs. $\mathbf{A}$ sequenti - longiore scta c longinq:o distinguendım.)

T. 2j. Polytr. Yuccacfol. ad. Loliis basi decurrenltibus, caule longissimo, ctiam prolifcro, r2rills ramoso occurrit.)

P. 2f. 1. 10. leg. caulc.

P. 27. Lincial. vulg. ad. Br. cxtinctorimm I.

- - ciliat. ad. obs. J'riori statmra major.)

- - ins. ii. Gymmostomum juleinarum, fo. liis ovatis concavis superioribus pilifer is; capsu. lis subscssilibus termimalibus operculo mi. nimo. 


\section{ET EMENDANDA.}

I1 rupestribus (Gött. - Autumuo, Vere. Br. pulvinato caespitulis, apocarpo capsulis si. nisile.?

- - - - truucat. ad. Antumno.

- - - - ovat. ad. Var.minor.)

P. 3ъ. Br. recurvatum, del.

- - lanccolat. ad. Var. minor brevicaulis.)

P.53. 1. 16. ad. et operculo obtuiso.

P. 34. controversum, ins. caps. suberectis, oper. culis obliruis.

P. 35. 1. 15. aquatic. ad. - ad aquas puras, ri. vulos scaturientes (Gott. Ileyder.) Autumno. Colore pallidiore, capsulis et foliis breviori. bus facile a seq. distingnitur.)

- - - ins. Br. crifurm, cilpsulis obliquis, foliis subulatis basi dilatatis undulatis demum tortilibus, cauliculis erectis Silzreb. spic. 1058 . Ad fossas (Lips. Schreb. -)

P. 37. 1. 17. Br. aciculare, leg. nigrricantibus. IIediv. II usc. ỏ. t. 33 .

P. 41. 1. 12. leg. fuliis confertis integris.

P. 42. ins. Br. aciculariforme, caule ramisque erectis fastigiatis, foliis lancolatis margine revoluto - patulis; capsulis ovato-oblongis. Dill. MTusc. 1.47. Jig. 30

Iu moutosis (Hercyu. Rehberg. EhrF. a Br. aciculari diversun : perist. deutibus capillaribus ad basin usque fissis erectis. Dill. cit. fig. potius 
potius hoc, quam Br. fastigiatuin indicare vi. den:r.)

F. 43. Br. Didymodon ad. - - ILedw. Musc. 1: ใ. 23 .

- - pallianm ad. (Gott. IIcyder.)

P. 4 i. l.25. lcg. superius.

2. ́f 7. 1. 10. ad. (2r. squarrosiun.)

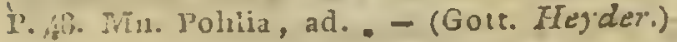

- bismim, ad. .

r. .o. \. jo ioco dedit $\operatorname{leg} e$ depiuxit.

P. (10. 1. 25.) leg. pediucrilis.

- - sulu hryoides, ad. obs. Iisdem in locis Get:. jeculiaris occurrit omnibus partibus

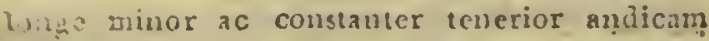
fijwir-? - unultis modis convenicus cun liss. irsinzala, trunculo brevissimo mbdecmuben. ti. foliis scalpelliformibus acutis, pinuatim duaricatis, thecis ccrmuis IIedw. Musc.4. r.' 80. $f: 5.1$.

P.56. 1, 26. leg. Bulbilli.

- - Il. sylvaticum, leg. Dill. Musc. t.8f. Jig. 6. Ihirds. angl. 2. 94. ad. Obs. In H. deris. culatn uneo folia ad leutem lauceolata, longe acuroinata, reticuli arcolne parallelae; in Ir. sylvatico, folia ovata acuta, reticuli arcolac laxac disjunctae. Nervis foliorum brevis api. ce bilidus.) 
P. 58. 1. 23ี. leg. pinnatie.

P. 60. 1.5. leg. a beque.

P. 62. 1. 37. ins. Atutunino.

P. 63. Hypn. uuciuatum, ad. Uercyn. Oderbr.m naguitudiue varisbili; surculis etiam pinnatis.)

P. 63. Hyopu. stellatum, ad. (Hercyn. Eithr7s.) .

- -1.31. leg. patulir.

P.67. 1.1. ins. Antumio, Vere.

- - - Hypn. attél. ad. " - Autumno.

F. 68. 1.4. 1ey. collectum.

P.69. 1.4. - Francon.

- - ins. Hypn. gracile, caule repente surcu. lis erectis ramisque fasciculatis reourvis tereti. Јн ; capsulis oblongis erectis. Hedw. musc. 4. t.6. (Pterisynaudr. gracile.)

In montosis (Hercyn. Eggeberg. Ifarh. - Folia oyato - lanceolata apice denticulata.)

P.71. Hypu. filiforme ad. . Heroyn.

P. 73. 1. 20. leg. vage.

P.74. 1.10. leg. nusalom.

P. $76.1 .26 . \mathrm{leg} \cdot 2 b^{\circ}$.

P.77. ins. Hypu. stramineum, surculis rectie fillormibus subranosis, Folis orato. Ianceolatis onervibus imbricatis Dicks. fasc. crype. 1. 6. t. 2. fig. 9 .

In locis paltudosis (Hercyn. Hamior. Ehrh.) 
P. 77. ins. Hypn. illeccbrum, surculis ramisquie ragis terctibus crectinsculis obtusis Schreb. spic. 2066 . Dill. MTusc. t. 40. fig. 46.

In 2ggeribus praris (Lips. Schreb. Setae brt. res uncia non longiores utplurimum curvae 1. c. - a me nondum obscrvatac.)

P. 78. Hypu. lluitans, ad. olss. sctis longissimis glabris, capsulis oblongis incurvis, peristom. dent. duplici scrie, caute $2 b$ adunco distin. guendum.)

P. 83. 1.12. leg. surculis.

- 1.26. leg. lunjus loci.

1. 85. 1. 88 . Icg. nt in sequente (Jung. tamarisci(oliz.)

T. 86. 1.9. leg. trmucis.

r. 87. Icg. Frondibus pinnatis, foliolis integris v:cl divijis.

- Jung. prllescens, ex lac subdivisione in antecedentem est transferenda.

P. 88. 1.22. Jung. Sphagui, 2d. obs. Folia fnsco. Intescertia.)

T.89. - - leg, dentatis.

J. gu. 1.27. leg. species

P. 93. 1. 29. lcg. fibrillosae - adglutinatae.

F. 94. 1. 4. ins. A.

r. 96. 1.3. lcg. minina.

- - ś́. - caverzosze. 
r. g3. lin. ult. ins. vix diaphana.

P. 99. lin. 10. ad. Lich. Raccidus, gelatinosiss, fol. membranaceis laxis obtuse lobatis atro viridibus, scutellis sparsis concoloribus dchar. Vet. Acad. Ficunll. 16. 1.1. Jig.4. medius quasi inter hoc et Coll. Vespertil. - rupestris.)

- -1.15 . ad. Gott.

- - 1.25. ins. Lich. discolor Achar. 1. c. t. 1.

fig. 5.)

P. 100. 1. 10. $1 \mathrm{cg}$. scutellis.

- 1. 17. ad. H. 1. Lich, marginalis Achar.

2. c. 8.

- 1.25. leg. fusco - sanguin.

P. $101,1.20 .1 \mathrm{eg} . \mathrm{C}$.

P. 102. 1. nlt. - discoideis.

P. 10.j. ius. Collema siutuxtumt, imbricatum simuato - laciuiatum truncatum denticulatum; scutellis concoloribus. Dill. Musc. t. 19. f. 35.

In terra, ad lapides.

P. 105. 1. 13. ad. H. 1. Lich. tenax Achar. l. c.

$$
\text { T. 1. Jig. } 1 .
$$

T. 110.1.7. leg. irrigatac.

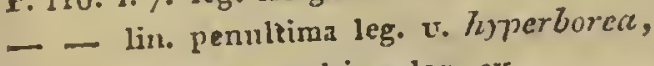

T.111. - antepenultima lcg. ex.

P. 129. leg. Tubercielosa.

P. 1331. 1.7. ad. obs. Iisdem in locis collecta a cel. Srrith, vid. Sketch, vol.2. 113.) P. $152 \pi$ 1. 25. Jeg. v2riis Enr.

Q 2 


\section{ADDRNDA}

F. 135. 1. 20. del. cum.

P. 25 g. 1. 16. ad. Licls. polliuarius Westr. Kongl. Vet. Handl. 16. 6.

P. 245. 1. 12. ad. Saltem 116 subspecies a L. saxa. sili distinguenda.)

P. 1/6. 1. 3. ad . 18.

D.147. 1. 16. 1eg. Accirbulicm.

P:15r. 1.8. ad. Saltem ut subspecies a L. phys. discingnenda.)

P. 265. 1.21. leg. glebulosae.

P. 172. 1. 10. - Glaucoma.

P. 175. 1. 1. - glebulosae.

P. 179. - - slebulosa.

- - 16. ad. Saltem 14 subspecies à priori dístinguenda.)

P. $182.1 .17 . \mathrm{leg}$ graunloso - rimosa.

P. 134. 1. 19. ad. H. 2. Lich. Concentricus Davies, in Trrens. lin. 2. 28. .

- 1.2e. del. nigrie.

P. 190. 1. 19. leg. distinguendse.

1. 192. 1. 27. ad. disco elabeute scrobiculatae.)

P. 194́. lin. ultima leg. microscopium.

T. 200. 1.5. del. aduatis.

I.3. ins. Inng. Dicronata, fronde simpliciten pinuata: foliolis conniventi bidentatis; apies. capanlifera. Schmid. icon. t.64. lig.z. 
In umbrosis rupestribus, ad terran. (Fron. des breves terrac affixae, periauthia foliolis plicatis cincta. Inter hano Jung. bifula oc. currit minutistima, perianthio snbquadrifido plicato angulato l. c. fig. \&. depicta, a me uon. dum ubservata.)

r. 8g. Jung. bicuspidata ad. Obs. Schmid. cit. fig. a Dillenii et meis exemplaribuz, rariori, bus minoribus hine inde 3.4 dentatis praeci. pre differt soliis.) 


$$
\text { E.R L A NGA E }
$$

IIIIS KV N I I ANIIAXIS。 
tortis, centro vel margine affixis frondibus variis: gclatinosis, coriaceis, umbilicatis, caulescentibus, loreis, filamentosis, foliaceis, tartareis, leprosis, perenuantibus, in propayines ( $\mathrm{Be}$ simina Neck.) varie efflorescentibus. Singula scutellata intra sulstantiam filamentoso - stupeam connata recondit semina.

34. Collema. Frondes diaphanae gelatinosae. Scutellae marginatae.

55. Peltigera. Frondes coriaceae. Sciztellae (Peltae) compressae margino adnatae.

36. Umbilicaria. Frondes foliaceae peltatae. Scutellae contortae.

37. Cladonia. Frondes caulescentes teretes intus cavae. Scutellae tuberculo. sac.

38. Stereocaulon. Frondes caulescentes teretes solidac. Scutellae tuberculosae, globosae.

3g. Usnea. Frondes loreae vel filamen. tosae. Scutellae radiatae aut nudae.

$$
x x
$$


40. I. obaria. Frondes lobatac, laciniatae, multifidae, erctae, depressae. Scurellae marginatac.

* Platisma. Fiondes lacunosae erectae, rel depressae.

* Physcia. Frondes in natac.

** Squamaria. Frondes subimbricata ingustatae.

**a* Placodium. Frondes subimbricatâe cruslaceac.

41. Psora. Crusta effgurata. Scutellae inarginatre, converae.

42. Vermcaria. Crusta aphylla: leprosa, tartareat. Scutellae marginatac, immarginatae.

Haec secundum octavam editionem Gen. pl. Lin. adnumerntis: Tremella, Ulva, Iucus, Conferva; Byfsus, andiunt: Ar.GAE; ab lis separandos existimavi LICHENES. 


\section{CATALOGVS}

Librorum ex Ilistoria naturali, Botanica; Medicina, et Clirurgia, quos propriis sumutibus impressit

IOANNES IACOBVS PALMI Bibliopola Erlangensis. 1796.

I o h a n n I a $k$ o b $\quad \mathbf{P}$ a $1 \mathrm{~m}$ Universitäts - Buchhändler in Erlangen hat nachstehende Werke ans der Naturge schichte, Botnnik, Medicin, Pliysik, und Chirurgie theils selbst verlegt, theils mis den Verlagsrechicn an sicls gekauft.

$$
1796 .
$$

Delii, D. H. F. principia diaetetica, Editio $2 \mathrm{da}$ et aucta. 8. $3781.15 \mathrm{kr} .4 \mathrm{Ggl}$. - primae lineae semiologiae pathol. 8. 2775 . $24 \mathrm{kr}$. $6 \mathrm{Ggr}$. - vom Preulsischen Blau und Blutlan. ge. 8. 1778 . 15 kr. 4 Ggr.

- vom aussezenden Puls, einigen audern Pulsarten und Angelegenlieiten des Herzens, eine scmiotisch practiscle Erlüu. terungsschift. $8.1784^{\circ} \quad 24 \mathrm{kr} .6 \mathrm{Ggr}$.

Gothliards Leitfaden für angehende Aer'zto und Wundärzte, Kranke zu prüfen und Krankheiten $z u$ erforschen, mit eirser 
Kranken - und Wittcrungsbeobachtungstabelle. gr. 8. 179 ! $\quad$ if. $16 \mathrm{G}_{\mathrm{g}}^{\mathrm{gr}}$.

Gottlards Tersuch eines vollstïndigen system. Lelitplans für Thicrarzueysclunlen. gr. 8. 1796.

45 kr. $12 \mathrm{Ggr:}$

Gramberg, D. G. A, de vera notione et cura moriornm primarum viarmu comment. cui alterum praenium ill. Acad. Imp. nat. cur. 17g2. decrevit. 8. maj. 2793.

auf Drukpap.
diart scripe.
$40 \mathrm{kr} \cdot 10 \mathrm{Grgr}$. $54 \mathrm{kr}, 14 \mathrm{Ggr}$.

Hildebrand dulcis mercurii landes, libellus medieus. 8. maj. $1794.12 \mathrm{kr} .5 \mathrm{Ggr}$. - über Arzneykunde oder Meilıodologie. 8. 1795.

- Lelırbuch der Mhysiologic. 8. 1796 6. 1 A. $30 \mathrm{kr}, 1$ Rith.

Hofers, F. I. Lehrsäıze des chirurgischen Verbands, 5 Theile mit 31 Kupfertafelu. gr. 8. 1790-92. 6 A. $50 \mathrm{kr} .4$ Fit. $12 \mathrm{Ggr}$ Hoffmatui, G. F. vegetabilia cryptogamica fasc. 1. et 2 dus cum 1b. tab. aen. 4. maj. 1787. แ. $99^{\circ}$ (jeder 2 Ris.) 6 A. 4 Rth. - de vario lichcuum usu. 4. maj. 1736: $20 \mathrm{kr}$. $5 \mathrm{Ggr}$.

- ohscrvationes botanicac. $4.78 .8 \mathrm{kr} .2 \mathrm{Gg}$.

- memoires sur l'utilité des lichens dans

- la medecine et dans les arts, avec. fig. gr. 8. 178-; 2 ll. $30 \mathrm{kr}$. 1 Rih. 16 Ggr.

- Dentschiands Flora oder botanisches

: Tascheubuch iter- Ialırgang. 1791. die 
erste bis 23 te Classe des Linmeischen Syst. cuth. mit 1. Tittelkupfer von Chodowieki gezeichuet und gestochen, und 12 Plinzcinkuptern. 12.

mit illum: Kpf. broch. 5 ll. 1 Rils. $16 \mathrm{Gg}$. nit scluw.Kpf. broch. 2 h. $6 \mathrm{kr}$. 1 Thl. $4 \mathrm{G}$. ungebunden und ohne alle Kupfer 1.l. $15 \mathrm{kr}, 20 \mathrm{Ggr}$.

- defsclben 2ter Ialurgang auf 17,75 . die Cryptogamie mit 14. illum. PÄanzen and 1. Tittelkupfer. 12.

broch. mit Futt. 4 f. '2 kr. 2 Rth. $10 \mathrm{Gg}$. brochirt mit Futteral und 2 Schreib. tafeln 4ח. $13 \mathrm{kr} .2$ Ril. $12 \mathrm{Ger}$ gebunden mit Futteral und Schreibtafelis $\quad 4 \mathrm{~h} .24 \mathrm{kr}$. 2 Rill. $16 \mathrm{Ggr}$. ungeb. olne alle Kpf. I $\mathrm{A} .15 \mathrm{kr}$. $20 \mathrm{Ggr}$. Beyde Jahrgänge für Ausländer sind ancle sovrohl mit französischen als englischen Titteln zu haben:

le méne livre:

la Flore de l'Allemagne on etrennes hotaniques, Partie 1. et 2 de, avec fig. illum. 12. $1791-96$. broclié

the Flota of Gernany or a botatical Pocket-Companion, Vol. I-II. 1791= 1796. broché

Hoppe enumeratio insectorum elytratorum circa Erlangan indigenarun, cum tab. 2en. 8. maj. 1795. iflum. 4o kr. 1n $\mathrm{Ggl}$. c. lig. nigris 2/f $\mathrm{kr}$. $6 \mathrm{Gg}_{\mathrm{g}}$. 
Klingsolur dc geoffraez inermi ejusque cortiça. 4. 17 \&̧.

$15 \mathrm{kr}$. \& $\mathrm{Grgr}$. Knigge, Th. de mentha piperitide, crim tals. aen. 4. $1780 . \quad 15 \mathrm{kr} .4 \mathrm{Ggr}$. in charta script. $24 \mathrm{kr}$. $6 \mathrm{G}$ is: Kobbs, I. A., wahre Ursache der Baum, trocknif8 der Nadelwälder durch die N2turgeschichte der Forlphalänc ervvicsen, und durch eigrene Versuche erörtert, nit drey illuminirten hupfern. gr. 4. 1793. 2 Il. 1 kìh. $8 \mathrm{Ggr}$.

Koelle, I, I. C. spicilorgium observationum de aconito, cum tab. aen. 8. niaj. 2783. $20 \mathrm{kr} .5 \mathrm{Ggr}$.

Liuné, Cax: à, Amoenilates academicae serı Dirs. variae pliysicae, med. botan. Fdit. 2da novissina et enendata cura Sclureberi, cum tab. aen. 10. Volumina 8. maj. $1787-.92$.

- ternini botanici. 8. maj. 1792. $8 \mathrm{kr} .2 \mathrm{G}$ err. Loschge de sceleto corporis lium. symetrico. 8. mil. 1795. in charta script. $36 \mathbf{k r} .9 \mathrm{Ggr}$. $45 \mathrm{kr} .12 \mathrm{Ggr}$.

Marc allgemeine Remerkungen ủber die Gifte vind ihre Wirkungen im mensch. lichen Köıper, nacl Browviscliem System. 8. $17.25 . \quad$ I $12 \mathrm{kr} .18 \mathrm{Ggr}$.

Mager, Tob. über die Gesetze und Modi. fication des Vïrmestoffs. 8. 91. 1 1. 16 Gg. Modeer, Ad. Bibliotheca helminthologica seu enumeratio auctormm, qui de vermibus scripserunt. 8. maj. 86. If. $16 \mathrm{Ggr}$. 
Ainller, Iol. Iteiur. über einige Fehler der körperlichen Erzielung der Kinder. 3. $179^{\circ}$. $3 \circ \mathrm{kr} .8 \mathrm{Ggr}$. Namen der Gattungen vor. Käferu in 5. Tabellen 36.

$24 \mathrm{kr} .6 \mathrm{Ggr}$.

Panzers Beyträge zur Geschichte der Inse. cten, siche Voet.

Rosenmunllers, Iol. Christ., Abbildungen nud Beschreibungen merksürdiger Ḥölen $11 \mathrm{~m}$ Muggendorf im bayreuth. Ober. lande für Freunde der Naumr u. Kunst, is Heft mit 2 gemahlten Kupfern. gl: Real Folio. 1796. $\quad 3$ A. $36 \mathrm{kr}, 2$ Rth. Sclimidel, C. C. Icones plantarum et analyses partium aeri incisae atque vivis co. lor. insiguitąe. Editio nova, Manipulus 1. et 2 dus sen 5 . Sectiones, cum 5o. tab. aen. pictis. fol. maj. 1793. (jeder Heft einzelin $6 \mathrm{~A} .43 \mathrm{kr}$. oder $4 \mathrm{Tith}$. $14 \mathrm{Ggr}$. 5.4 H. 22 Rth. $22 \mathrm{Gg}$. - ejusdem hibri Manipuhs IIItins. c. Tab. LI.IXXXV. 1795. 2n A. 15 Rth. 8 Ggr. - Vorstellungen merkwürdiggez Versteinerungen mit Anmerkmogen 4 Hefte mit 24 illuminirt. Kupfertafelu. gr. 4. 1795. ${ }_{15} 5$ h. 10 Rith.

- iter per IIelvetiam, Galliam et Gcrmaniae partem 1773 et 74 . cum 2 tab. pictis rei botau. 4. maj. 1795.

2 fl. $50 \mathrm{kr}$. I Ril. $16 \mathrm{Ggl}$. Schoepffi, I. D. Historia testudinum iconibus illustrata, particula I. K. cont. 
Tab.I-XXV. et Text. A - O. cum praefat. 4. $11 \mathrm{aj} .1702 \cdot 9^{5}$.

illuminit $18 \mathrm{~A} .54 \mathrm{kr}$. I2Rth. I/4 Ggr.

- idem liber mit schwarzen Kupfein Io $1.50 \mathrm{kr}$. 7 Rits.

- Naturgeschichte der Sclildkróten voin Verf. selbst übcrsczt, $\mathbf{r}-5 \mathrm{r}$ Heft euth. Tab. I- XXV. und Text A - P. gr. 4. $x 79^{2} \cdot 95$

mil illum. Kitr. Igh. $24 \mathrm{kr} .12 \mathrm{Rth}$. $12 \mathrm{Gg}$. mit schw. Kpf. anf Schweizer Papici 11 月. $50 \mathrm{kl} .7$ Fith. 16 Gy. Text anf Drukpapicr mit schw. Kupfern auf deutschem Papier 7 A. 4 Keh. Von allen 5 Heft. compl. sind Exempl. auf holl. Postpap. mit fein gemahlt. Ku. pferu für 18 Reli. zu habcu.

- Matcria medica Americana. 8. maj. 86. $45 \mathrm{kr} \cdot 12 \mathrm{Grr}$

- von der Wiîrkung des Moluusafts in der Lustscuclie, nelist nudern zur Arzneygohluheit und Natulohre zehörigen Henbachtmigen Nordamerika bet. mit ciner Vorrede vou Delius. gr. 3. $178 \mathrm{r}$. $15 \mathrm{kr} .4 \mathrm{Ggr}$.

- Bcytrigge zmx mineralogischen Kenut. nifs vou Nordamerika. S1.8. 1787. $45 \mathrm{kr} \cdot 12 \mathrm{Ggr}$.

- Beschreibung seiner Reise durch cinige der mittlcru and sülliclien vereinigten Nordamerikail. Staaten rach Ostflorida und der Beliama. Insel, unternom- 
men in den Iahren 1783. 11. 84. 2 Thle. g1:8. 1788 . 5月. 3 Rth. 8 Ggr. 3 warz, Ol. olsservationes hotanicae quibus plantae Indiae occidentalis aliaeque systematis vegctab. Ed. XIV. illustrantu1 earumque characteres cmendantur, cum XI. tab. aen. 8. maj: 179r.

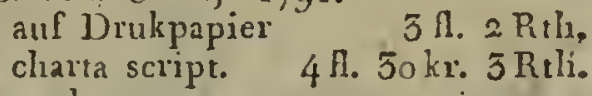

- Icones plantarum novarmm minusque cognitarum Indiae occidentalis, Fasc. $x$. Sectio I. cont. Tab. I. VI. pictas fol. maj. 1794. 5 H. 54 kr. 2 Rth. $14 \mathrm{Ggr}$.

Thomann Geschichte einer mcrastatischein Angenentzïndung, mit r. Kinper gr. 4 . Würz.b. 1796.

Thunberg, C. P. prodromus plantarim Capensium in promontorio bonac spei Africes, Pars I. cum 3. lig. aen. gr. 8. Upsaliae 1794 .

Udmarini, Is. novae insectorum species, Editio $2 \mathrm{da}$ curante Panzero, cum $2 \mathrm{fg}$. 4: 1793." 40 kx. 10 Gg: Vocts, Iob. Ens. Beschreibungen und Ah. bildungen haxtschaligter Insecten, übersezt, mit der Synonimie vermehrt und mit Commentarien erlàtert von G. IV. F. Panzer, des Originals ir Theil oder der deutschen Ausgabe I uud 2tex Theil, in 27 Ausgaben mit 5o illum. Kupfern. gr. 4. 1793 . 17 月. I1 Puh. $3 \mathrm{Ggr}$. - defselben 2r Theil des Originals, ndev der deutschen Ausgabe 35 und 4ter Th. 
.mit 50 illum. Kupfertafelu, gr. \{. 17033 . 1706. 20月. 15 Rul. 8. Ggr.

- defselben 5tar Theil oder G. TT. T. Panzers licyträge. zur Inscctengesclichte mit illum. Kupf. Iter Theil ssie Ansga. be end. Tab. I - VI. Text A - C. gl. \&. 1795. 2 ก. $15 \mathrm{kr} .1$ lith. $12 \mathrm{Ggr}$. vendts, D. Fr., Nacluichten von dem Kranken - Institut zu Erlangen, 6 Stïcke. gr. 8.1786. $45 \mathrm{kr}, 12 \mathrm{Ger}:$ - de Celuribus remittentibus semestris lii. berui. 3. 5796 .

Vulfen, Abts Xav., Abhandl. voin Kärnthenschen pfanenschweifigen Ileluintholith, oder dem opalisirenden Musclaclmarmor, mit 12 illumin. Kupfertafeln, nebst $\mathrm{dem}$ Grundrirs des Gebirges gir. 4. $\mathbf{I}-9 . \overline{3}$. 7 Il. 4 Tith. 16 Gegr. Dic übrigen vorräthigen Z.eichnumgen, viel. che olmugefahr noch 28 Kupfertafelu ansfizl. len werden, sind nuter der Künstler Hăn. den.

Mediciuische Iandbibliothek oder alplia. betisches Verzeichnifs de1er Büclıer aus der Arzneygelahnheit, Chirnrie, Anato. mie, Botanik, Naturgeschichte, Clicmie, Apothekcrkunst etc. mit Preifsen. 8. 1783. I I. $16 \mathrm{Gg}$. 
5 


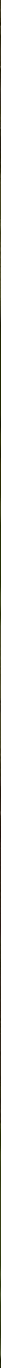





